\title{
Assessing anthropogenic noise impacts and relevant soundscape cues for marine invertebrates: leveraging squid and coral reefs as model systems
}

\author{
by \\ Ian Thomas Jones \\ B.S., Marine Science, University of Maine, 2015 \\ Submitted to the Biology Department in partial fulfillment of the requirements for the degree of \\ Doctor of Philosophy \\ at the \\ MASSACHUSETTS INSTITUTE OF TECHNOLOGY \\ and the \\ WOODS HOLE OCEANOGRAPHIC INSTITUTION
}

September 2021

(C) 2021 Ian Thomas Jones. All rights reserved

The author hereby grants to MIT and WHOI permission to reproduce and to distribute publicly paper and electronic copies of this thesis document in whole or in part in any medium now known or hereafter created.

Signature of Author

Joint Program in Oceanography/Applied Ocean Science and Engineering Massachusetts Institute of Technology and Woods Hole Oceanographic Institution

Certified by

August 5, 2021

Dr. T. Aran Mooney Associate Scientist with Tenure

Thesis Supervisor

Accepted by

Woods Hole Oceanographic Institution

Dr. Mick Follows

Chair, Joint Committee for Biological Oceanography

Woods Hole Oceanographic Institution 


\title{
Assessing anthropogenic noise impacts and relevant soundscape cues for marine invertebrates: leveraging squid and coral reefs as model systems
}

by

\section{Ian Thomas Jones}

Submitted to the MIT Biology Department and MIT-WHOI Joint Program in Oceanography/Applied Ocean Science and Engineering on August 5, 2021, in partial fulfillment of the requirements for the degree of Doctor of Philosophy in Biological Oceanography.

\begin{abstract}
Sound is utilized by marine animal taxa for many ecologically important functions, and these taxa are vulnerable to adverse effects of anthropogenic noise on hearing and behavior. However, little is known about marine invertebrates' responses to anthropogenic noise, and the ambient environmental sounds ("soundscapes") they detect and respond to. Most acoustic studies report sound pressure (detected by mammals and some fish), but few report particle motion, the back-and-forth vibratory component of sound detected by marine invertebrates. I investigated invertebrate use of and response to sounds in two facets: 1 ) behavioral responses of longfin squid, Doryteuthis pealeii to anthropogenic noise, and 2) particle motion of coral reef soundscapes in the U.S. Virgin Islands. In laboratory-based experiments I exposed $D$. pealeii to construction noise originally recorded from an offshore wind farm. I found significant increases in squids' alarm responses and in failed prey capture attempts during noise. Conversely, noise exposure had no significant effects on reproductive behaviors of groups of $D$. pealeii, indicating high motivation of these squid to reproduce during this stressor. Collectively, these experiments revealed the importance of considering behavioral context in studies and regulatory decisions regarding invertebrates' susceptibility to anthropogenic noise impacts. In studying coral reef soundscapes, I reported particle motion trends over several months for coral reefs varying in habitat quality, including coral cover and fish abundance. I found acoustic properties over which particle motion closely scaled with pressure, and others over which it did not. I compared soundscape data with particle motion hearing thresholds, and found that invertebrates may only detect high amplitude and low frequency transient sound cues on reefs, such as those produced by fishes. My research bring new insights on natural and anthropogenic sound cues detectable by marine invertebrates, and how and when invertebrates will be vulnerable to anthropogenic noise pollution.
\end{abstract}

Thesis Supervisor: Dr. T. Aran Mooney

Title: Associate Scientist with Tenure

Biology Department

Woods Hole Oceanographic Institution 


\section{Acknowledgements}

My graduate work was funded in part by the US Department of Interior, Bureau of Ocean Energy Management Environmental Studies Program through Interagency Agreement Number M17PG00029 with the U.S. Department of Commerce, National Oceanic and Atmospheric Administration (funding to Aran Mooney and Jenni Stanley). My work was also supported by the NSF Biological Oceanography award OCE-1536782 (funding to Aran Mooney). I received tuition and stipend support from the National Science Foundation Graduate Research Fellowship Program [Grant No. 2388357]. The Academic Program Office at the Woods Hole Oceanographic Institution provided tuition and stipend support as well as travel support. The MIT Student Assistance Fund, the Aquatic Noise 2019 Organizing Committee, and the Acoustical Society of America also provided travel support.

I have many people to thank who in various ways, big or small, professionally and personally, all played an important part in helping me reach this point in my research career.

Words can't fully express how much I value everything my research advisor, Aran, has done for me these past years. Aran, thank you for believing in me and giving me the space and encouragement to explore and develop my abilities, and confidence, in conducting independent research. At every step of the way, from figuring out Thesis topics to scientific writing, you've been nothing short of immensely supportive, providing a constant flow of great ideas and constructive feedback. Literally every time I've spoken to you about an issue, whether it was about data analysis, field work logistics, or otherwise, I've felt better after talking with you than before. Thank you also for always encouraging a healthy work-life balance and reminding me to take breaks. On top of research mentorship, you've played a huge role in helping me network, especially at conferences in my earlier graduate years. Throughout all this, you've always made me feel welcome and an equally valued member of your lab.

Thank you to my Thesis committee, Roger Hanlon, Stefan Helmreich, Laela Sayigh, and Dan Zitterbart, who have provided frequent and highly valuable guidance and feedback throughout my dissertation.

You have all helped me focus on and convey both the fine technical details and broader implications and applications of my research.

I want to thank the WHOI Summer Student Fellowship (SSF) staff, including Kama Theiler, and my SSF mentors Ann Tarrant and Amy Maas for the very positive and engaging research experience I had at WHOI as an undergrad. Your enthusiasm and dedication to this program and as mentors solidified my intent to pursue grad school at WHOI in the first place.

Thank you to everyone in the WHOI Academic Programs office and MIT JP office who consistently made me feel welcome and valued as a student, especially Julia Westwater, Lea Fraser, and Meg Tivey, Christine Charette, and Kris Kipp. You all ensured I had housing, travel, funding, and other essential resources, and top of these responsibilities you have always been there to address any miscellaneous student issues and concerns. My progress as a grad student would not have been possible without the passionate work you put into the Joint Program. Thank you as well to Gaynor Andrews for all your administrative assistance to folks in the MRF.

Looking back to my first summer conducting field work in St. John with the Mooney Lab, I have many student and faculty collaborators to thank for acclimating me to field work in a research field that was brand new to me, and maintaining a fun research environment in later field seasons. Thank you to Matt Long, Joel Llopiz, Amy Apprill, Ashlee Lillis, Laura Weber, Alexis Earl, Jessica Perelman, Jessica Dehn, Cynthia Becker, Jason Dinh, Morgan Bennett-Smith, Paul Caiger, Rod Catanach, Camille Pagniello and all 
the guest researchers and students involved in the Coral Chorus project whose guidance, enthusiasm, and encouragement helped me adjust to this work in my first field season and made it even more enjoyable in later field seasons. Thanks to Travis Hamlin who helped keep me sane and our boat operational amidst engine and weather issues. Thank you to the staff of the Virgin Islands Environmental Resource Station and National Parks Service at St. John who accommodated and permitted this research. Thank you especially to Justin Suca (leader of "Team 'Murica", among other names) who taught me so much about fish ecology and boating, including how to survive using the ill-fated $R / V$ Sonic Youth, and for on more than one occasion preventing me from getting stranded on it. Justin, not only have you been a valuable peer to me as we were adjusting to PhD life (and trying to figure out basic acoustic data processing), you've been a great friend from the very start.

Thank you to Casey Zakroff who immediately made me feel welcome as a newcomer to the Mooney lab and whose advice helped me get started with squid husbandry. I always enjoyed chatting with you in the MRF Fish Bowl whether it was about how our work was going, exchanging advice on data visualization, or navigating WHOI bureaucracy.

Thank you to Jenni Stanley, who has always been an absolute blast to conduct research with and whose mentorship has made a huge positive impact on how I design bioacoustic experiments and report acoustic measurements. You never failed to remind me of the rigor and broader importance of my research whenever I doubted it.

Thank you to all the students who have played critical roles assisting with squid behavior experiments and analyses and, and roles as co-authors on my manuscripts, including Hadley Clark, James Peyla, Zongchang Song, and Madison Schumm. These projects wouldn't have been possible without your help, and your ideas helped shape the course of our experiment designs and analysis methodologies. Thank you to Sophie Ferguson for helping with boat noise auditing of soundscape data. Thank you to all Mooney Lab members, WHOI guest students, volunteers, and researchers who also assisted me countless times with trucking squid from MBL to WHOI, collecting killifish, and squid husbandry.

Thank you to the faculty researchers who have also served critical roles as advisors and collaborators as I was figuring out how to record and report particle motion. Thank you to Julien Bonnel for your advice on visualizing and describing particle motion fields in my squid experiment tanks. Thank you to Walter Zimmer and Michael Gray who guided me through much of my reef soundscape particle motion analyses. Michael Gray also helped with the hydrophone array design, and constructed the housings for the accelerometers I've depended on for all my lab-based experiments at WHOI.

Thank you to the many postdocs, JP students, guest students, and investigators of the Mooney Lab who helped me along the way by providing advice on my progress at lab meetings and presentations, and who generally made the lab a fun, engaging, and welcoming atmosphere, including Adam Smith, Amy Van Cise, Andria Salas, Ashlee Lillis, Francesco Caruso, Frants Jensen, Matt Long, Seth Cones, Tammy Silva, and Nadège Aoki.

I also wish to thank Youenn Jézéquel and Jenny Panlilio with whom I was able to work on really cool projects that were part of their dissertations, and which enriched my graduate experience. I hope we are able to collaborate again in the future.

Thank you as well to Grace and Saniya whom I had the fortune of mentoring during their high school science fair projects. Your enthusiasm for scientific discovery helped boost my own enthusiasm and my passion for mentoring. 
I cannot thank Rick Galat, Ed The Plumber, and WHOI facilities staff enough for all their hard work maintaining the Environmental Systems Lab (ESL) at WHOI, and for helping me set up tanks and ensuring I had the seawater requirements I needed. Thank you to the MBL Marine Resource Center director Dave Remsen, coordinator Scott Bennet, Dan Calzarette, and Gemma captain and crew members Bill, Bill, and Bill for providing me (many) squid. Thank you as well to Arthur Newhall, Gopu Potty, and their colleagues who provided field acoustic data of pile driving noise. None of my squid experiments would have been possible without all of you. Thank you as well to Dave Bailey who helped orient me to the ESL and was patient with me when I made mistakes (like shutting off the wrong circuit breakers).

I am so thankful to all the members of GLOW, who have always made me feel welcome and safe at WHOI and in Woods Hole, and helped keep me actively and socially engaged in the local LGBTQIAP+ community.

Thank you to my fellow JP students, who across cohorts have always maintained a fun, inclusive and active social community even when I've been spatially removed from them. I'll fondly remember our social hours, tropical parties, Jelly Talks, winter retreats, barbecues, and other events. And thank you to every JP student who has acted to push for a more diverse and equitable WHOI and Joint Program.

Thank you to all the WHOI dogs who brightened my work days.

I also want to thank all of the friends I've made the last few years outside the MIT-WHOI social sphere who have helped me survive my PhD by greatly enhancing my social life. Thank you to the Furry Fandom and Boston Pets who have given me much needed outlets of escapism from the everyday challenges of being a human PhD student. Interactions with these communities really boosted my confidence in social situations; a change which has carried over to my professional life.

Thank you to dance music, which gotten me through many hours working with MATLAB, measuring tank acoustics, and writing. Thank you to the Anjunafamily, Anjunimals, and E.C.F. DJs who have kept me sane through our shared passions for electronic music and DJing. And thank you to all the music artists who unknowingly produced the soundtrack to my PhD.

Thank you to my family and especially my parents for always having confidence in me, and always being enthusiastic about my work. I can't imagine getting as far as I have without your constant

encouragement and support. Thank you to my brother who continues to inspire me with his creativity and love of nature.

Finally, thank you to my partner Evan, who has been with me on this path since the start of undergrad. You have always rooted for me and been immensely supportive of my science endeavors. Thank for always reminding me when I get stressed that things will be okay, and thank you for your patience and support as I worked through the most challenging parts of my dissertation. I love you and I can't imagine being on this journey without you. 


\section{Table of Contents}

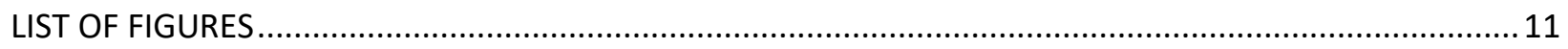

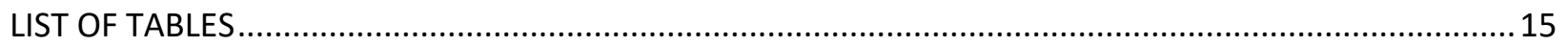

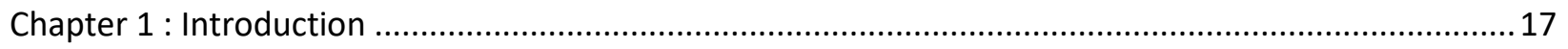

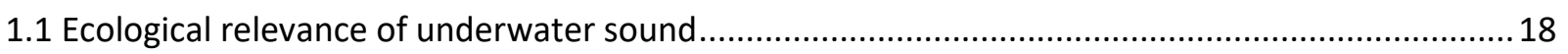

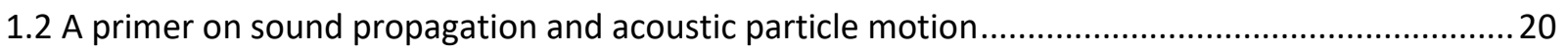

1.3 Anthropogenic noise pollution from an invertebrate perspective ...............................................22

1.4 Cephalopoda as a model taxon for invertebrate bioacoustics .................................................... 24

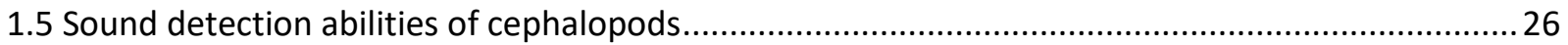

1.6 Coral reefs as model ecosystems for marine soundscape studies ............................................... 27

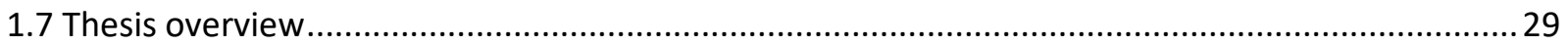

Chapter 2 : Impulsive pile driving noise elicits alarm responses in squid (Doryteuthis pealeii) ................. 33

Ibstract

Chapter 3 : Changes in feeding behavior of longfin squid (Doryteuthis pealeii) during laboratory exposure

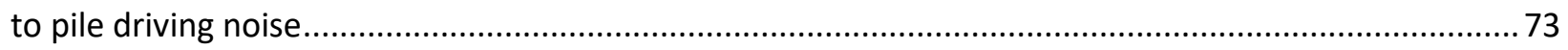

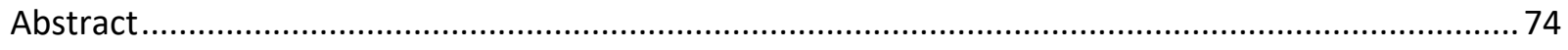

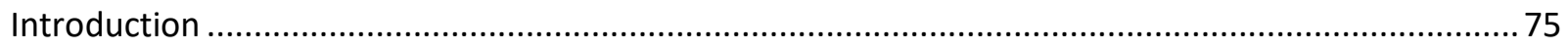

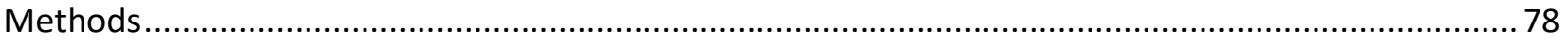

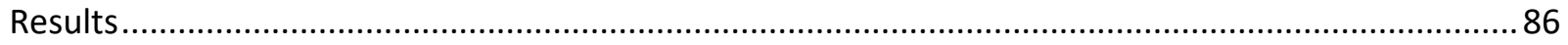

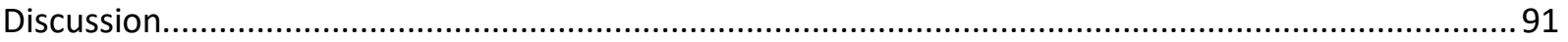

Chapter 4 : Context is key: squid reproductive behaviors and spawning withstand wind farm pile driving

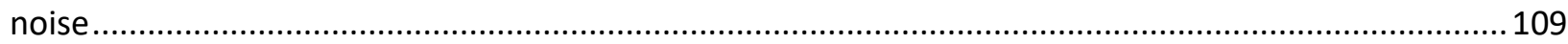

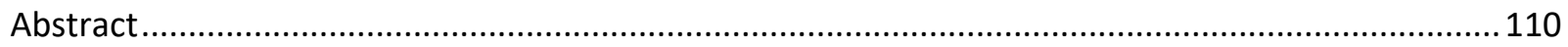

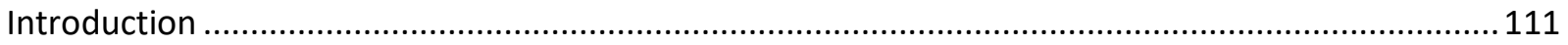

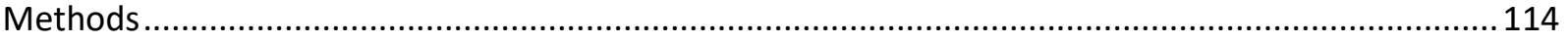

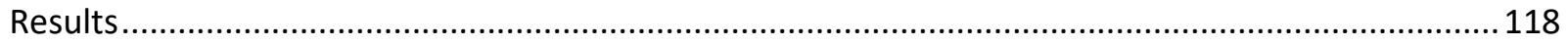

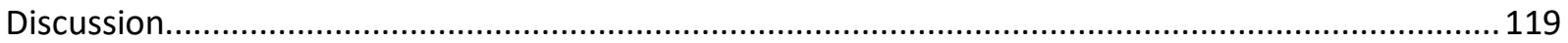

Chapter 5 : Natural cues for invertebrate and fish hearing: particle motion measurements on reefs.... 127

Abstract. 
Introduction 129

Methods 132

Results 137

Discussion. 142

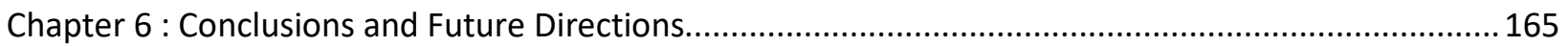

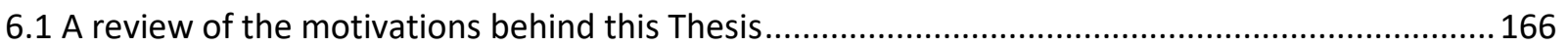

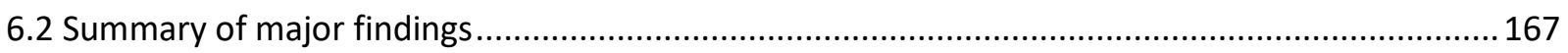

6.3a Context-dependent responses of squid to noise: broader ecological and management implications 168

6.3b Future directions......

6.4a Particle motion of coral reef soundscapes and their detectability by invertebrates and fishes: broader ecological and management implications 173

6.4b Future directions

6.5 Recommendations for lab and field-based particle motion measurement

6.6 Final thoughts 177

Appendix A: Chapter 2 Supplementary Materials . 179

Appendix B: Chapter 3 Supplementary Materials 183

Appendix C: Chapter 4 Supplementary Materials 193

Appendix D: Chapter 5 Supplementary Results. 199

Appendix E: Complexities of tank acoustics warrant direct, careful measurement of particle motion and pressure for bioacoustics studies

Appendix F: Example MATLAB code used to calculate particle acceleration from accelerometers and hydrophone arrays 225

BIBLIOGRAPHY 237 


\section{LIST OF FIGURES}

\section{CHAPTER 2}

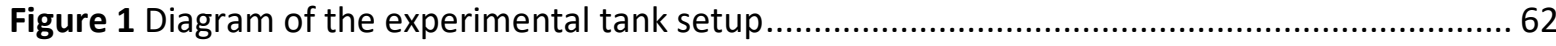

Figure 2 Example images of squid alarm responses observed during noise exposure .................... 63

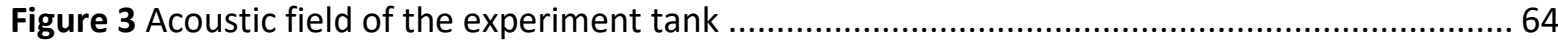

Figure 4 Received sound levels for the first three noise impulses ............................................. 66

Figure 5 Alarm response proportions for each response type and trial type ..............................67 67

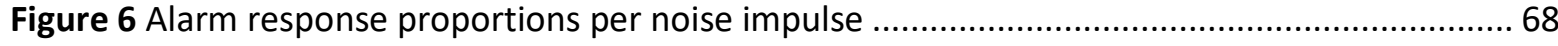

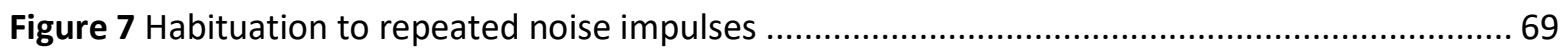

Figure 8 Body pattern change responses separated by groups of chromatic components ............... 70

Figure 9 Amplitude dependence of alarm responses to noise ................................................... 71

\section{CHAPTER 3}

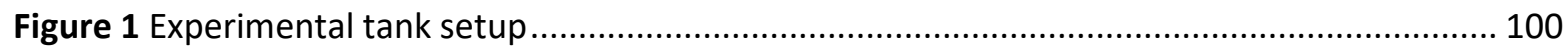

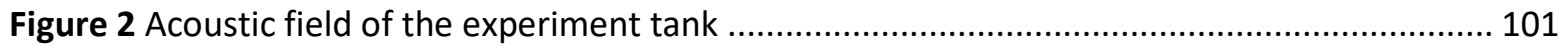

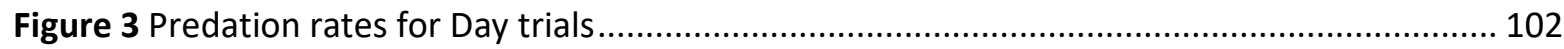

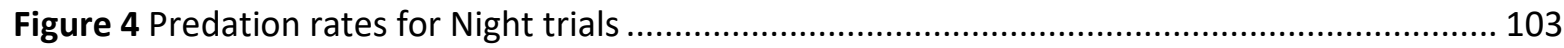

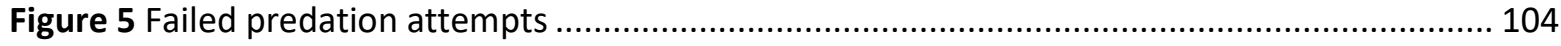

Figure 6 Squid and killifish hearing thresholds compared with noise in the experiment tank ........ 105

Figure 7 Squid predation latency versus killifish locomotion ..................................................... 106

Figure 8 Alarm response proportions for squid before and during playback.............................. 107

Figure 9 Habituation of alarm responses for squid in feedings trials........................................ 108

\section{CHAPTER 4}

Figure 1 Mate guarding behaviors of large and small male squid............................................. 123

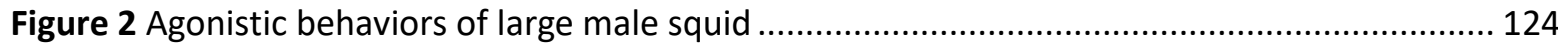

Figure 3 Relative response rates of squid to noise compared across Chapters $2-4 \ldots \ldots \ldots \ldots \ldots \ldots . . . . . . .125$ 


\section{CHAPTER 5}

Figure 1 Map of study sites and image of acoustic recorder deployed......................................... 153

Figure 2 Daily averages of particle acceleration and pressure .................................................... 154

Figure 3 Correlation plots of 3D particle acceleration and pressure .......................................... 155

Figure 4 Diel periodicity of 3D particle acceleration ............................................................... 156

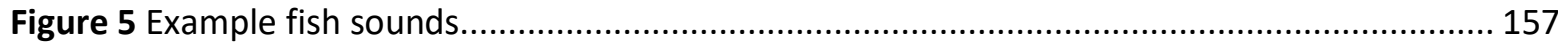

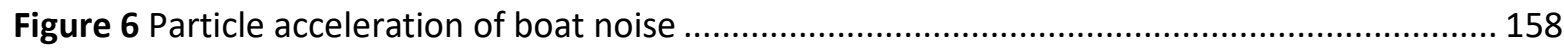

Figure 7 Invertebrate and fish audiogram data compared with soundscape data ....................... 160

Figure 8 One-octave band levels of soundscape data ......................................................... 162

Figure 9 Strength of 3D particle acceleration diel trend correlated with habitat quality data ........ 163

\section{APPENDIX A}

Figure S1 Alarm response rates per noise during and prior to playback ................................... 181

Figure S2 Body pattern change responses separated by individual chromatic components........... 182

\section{APPENDIX B}

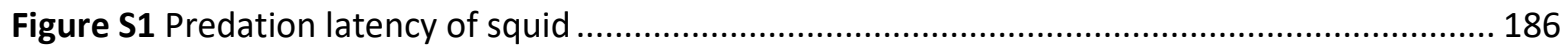

Figure S2 Proportions of alarm response types during Day and Night trials.............................. 187

Figure S3 Alarm response rates per noise during and prior to playback ................................... 188

\section{APPENDIX C}

Figure $\mathbf{S 1}$ Acoustic field of the experiment tank 195

\section{APPENDIX D}

Figure S1 Sensitivity analysis of amplitude error for hydrophone arrays .................................. 200

Figure S2 Residual plots for regression analysis of particle acceleration and pressure ................. 201

Figure S3 Finite-difference power spectra compared with plane wave approximations ............... 202

Figure S4 Diel periodicity of 1D particle acceleration and pressure............................................ 203

Figure S5 Spectrograms of 1D particle acceleration and pressure for an example boat pass ......... 204 
Figure S6 Diel trend strength of pressure correlated with habitat quality data

\section{APPENDIX E}

Figure 1 Image of the experiment tank setup ..................................................................... 218

Figure 2 Spatial maps of broadband pressure and particle accleration in the tank....................... 219

Figure 3 Pressure and particle acceleration versus distance from the speaker ............................. 220

Figure 4 Spectrograms of pile driving field recordings and in-tank playbacks .............................. 221

Figure 5 Spatial maps of narrowband pressure and particle accleration in the tank ..................... 222

Figure 6 Waveforms and cross-correlation revealing effects of reverberation in the tank ............. 223 


\section{LIST OF TABLES}

\section{CHAPTER 2}

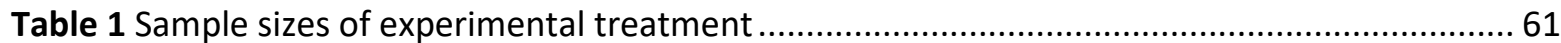

\section{CHAPTER 3}

Table 1 Sample sizes of experimental treatments.

CHAPTER 5

Table 1 Visual survey data of reef habitat quality..... 152

\section{APPENDIX B}

Table S1: Detailed statistical results 189

\section{APPENDIX C}

Table S1 GLMM results of mate guarding by large males. 196

Table S2 GLMM results of mate guarding: trials where only large males mate guarded 196

Table S3 GLMM results of agonistic chases by all large males 197

Table S4 GLMM results of agonistic lunges by all large males 197 
CHAPTER 1 : INTRODUCTION 


\subsection{ECOLOGICAL RELEVANCE OF UNDERWATER SOUND}

Sound is a vital sensory cue for marine animals including mammals, fishes, and invertebrates. Each of these groups utilize sound for a myriad of ecological functions, including detection and avoidance of predators, foraging, navigation, and communication with conspecifics in reproductive or competitive contexts (Au \& Hastings, 2008; Hughes et al., 2014; Ladich, 2019; Popper et al., 2001; Radford, Stanley, et al., 2011; Tricas \& Boyle, 2014; Webster et al., 1992). Ambient sounds from natural habitats also induce settlement behavior in many pelagic invertebrates and fishes (Gordon et al., 2019; Lillis, Apprill, et al., 2018; Lillis et al., 2013; Stanley et al., 2012; Suca et al., 2020).

Hearing in marine taxa is a basal sensory mechanism. It likely evolved not for communication, but rather to detect acoustic cues and gain information about ones' surrounding environment by listening and constructing an "auditory scene" (Fay, 2009; Popper \& Fay, 1993). This auditory scene refers to the detection, segregation, and identification of individual sound components in an environment.

Collectively, these sounds make up the ambient "soundscape". A natural soundscape (i.e., without human activity) consists of biological and geophysical sounds. Although biological sounds may arise from signals purposefully produced by animals to communicate, many sounds are unintentionally produced as byproducts from feeding, swimming, or other activities (Lobel et al., 2010). Underwater geophysical sounds arise from sources such as wind and wave motion, storms, or earthquakes. A wide variety of biological and non-biological sounds are naturally available in the oceans, and marine animals across taxa have evolved to detect these sounds to gain ecologically relevant information about their habitats and other organisms.

Historically, research on sound detection, production, and acoustic ecology has largely concentrated on marine mammals. Fish have received less focus, and invertebrates remain the least studied group (Hawkins et al., 2015). The dearth of acoustic research on invertebrates is surprising considering they represent large proportions of total biomass and species diversity in marine ecosystems (Costello et al., 2010). In recent decades, appreciation and understanding of sound detection and use by marine invertebrates has grown and more research has quantified 
sound-mediated abilities in diverse taxa, including mollusks, arthropods, and cnidarians (e.g., Jézéquel et al., 2021; Lillis et al., 2016; Mooney et al., 2010; Packard et al., 1990; Roberts et al., 2015b; Stanley et al., 2011). Many details about the hearing abilities and ecological functions of hearing in these taxa remain unknown, relative to what is known for fishes and mammals (Popper \& Hawkins, 2018). Little is understood regarding how noise pollution from anthropogenic sounds will adversely affect the physiology and behavior of marine invertebrates, their sound-detection abilities, and how such changes may lead to populationlevel consequences (Hawkins et al., 2015). The ocean is rapidly changing, in part because of increasing human-produced noise. It is timely to conduct research focused on model invertebrate species and habitats wherein the sound cues available to these species, and responses of invertebrates to sound can be thoroughly quantified and better understood.

All fishes and many invertebrates detect particle motion, which refers to the "back-andforth" vibratory movement of particles in a sound field. Conversely, marine mammals and some fish (with compressible air cavities such as an air-filled inner ear) detect sound pressure, but pressure detection has not been described in marine invertebrates. Invertebrates, which generally lack compressible, air-filled cavities, appear only sensitive to particle motion. Until recently, a lack of accessible instrumentation for quantifying acoustic particle motion underwater, and limited understanding or agreement among researchers about how best to do so, prevented particle motion from being reported in many studies. Fortunately, scientists are now quantifying particle motion more frequently. This is in part thanks to a greater availability of commercial instruments and an increased understanding of the necessity to measure particle motion when examining the acoustics of fishes and invertebrates (Jesus et al., 2020; Kaplan \& Mooney, 2016; Nedelec et al., 2016; Wahlberg \& Westerberg, 2005). These advancements are relatively recent and the underwater particle motion of many ecologically relevant cues and anthropogenic noise sources has yet to be well-quantified and understood (Hawkins \& Popper, 2018; Nedelec et al., 2016; Wahlberg et al., 2008). 


\subsection{A PRIMER ON SOUND PROPAGATION AND ACOUSTIC PARTICLE MOTION}

Any object that moves a fluid medium will propagate sound and generate an acoustic field. An acoustic field is made up of vibrating "particles", defined as the smallest parcel of fluid that represents that fluid's mean density (ISO/DIS, 2017). These particles oscillate in a linear or elliptical pattern with no net transport, and this movement is referred to as "particle motion". Motion of groups of particles will lead to alternating areas of high pressure (compression, where particles move closer together) and low pressure (rarefaction, where particles move further apart), resulting in propagation of a pressure wave. At a given point in space, pressure is a scalar quantity with equal magnitude in all directions, and particle motion is a vector quantity with a magnitude and a direction. Particle motion can be quantified as displacement, velocity, or acceleration. Acceleration is considered the most relevant transduction stimulus for acoustic sensory structures of invertebrates and fishes (Packard et al., 1990; Popper \& Hawkins, 2018; Popper et al., 2001). I selected acceleration to describe particle motion throughout this Thesis. I also quantified sound pressure to allow comparison with other studies, and to better understand relationships between particle motion and sound pressure in the lab and field environments in which my research took place.

The relationship between particle acceleration and pressure and the attenuation of these quantities with distance from the sound source are dependent on multiple variables, including sound frequency, radiation pattern (e.g., monopole versus dipole), depth, and proximity to boundaries (e.g., walls, sea surface, or seafloor). Away from boundaries and in relatively deeper water, sound fields can be approximated as a "plane waves" in which the wave fronts are plane surfaces perpendicular to the axis of propagation. When such conditions are met, pressure and particle acceleration will scale predictably with each other, and the magnitude of acceleration can be calculated from pressure as:

$$
a_{p w}=\frac{2 \pi f * P}{\rho c}
$$

where $f$ is the frequency $(\mathrm{Hz}), P$ is sound pressure $(\mathrm{Pa})$ at a given frequency, $\rho$ is the seawater density $\left(\mathrm{kg} \mathrm{m}^{-3}\right), c$ is the sound speed $\left(\mathrm{m} \mathrm{s}^{-1}\right)$, and $a_{p w}$ is the particle acceleration $\left(\mathrm{m} \mathrm{s}^{-2}\right)$ of a plane wave at a given frequency (Nedelec et al., 2016). The range from a sound source at which 
this relationship approximately holds true is termed the "far field". Closer to a sound source in the "near field", the ratio of particle acceleration to pressure is greater than that predicted by Equation 1, and wave fronts are more curved instead of planar (Rogers \& Cox, 1988). The distance at which the near field transitions to the far field is often approximated as $\lambda / 2 \pi$, where $\lambda$ is the wavelength of the sound at a given frequency $(\lambda=c / f)$. Thus, the near field extends to greater distances for lower-frequency sounds. Note that there is no single exact boundary; in reality a gradual transition exists between near and far field propagation (Kalmijn, 1988).

The spatial range of the near field also depends on whether the sound radiates as a monopole, dipole, or multipole. Monopole sounds radiate equally in all directions (also known as "spherical waves"), dipole sounds have a figure-eight pattern with two lobes of maximal pressure and orthogonal areas of minimal pressure, and multipole sounds have multiple lobes. Dipole and multipole sounds thus have more spatial variability in pressure and particle acceleration magnitudes than monopole sounds. Natural sounds produced and detected by invertebrates and fishes are thought to exist more commonly as multipoles or dipoles in the near field than as monopoles (Kalmijn 1988).

Close to boundaries, in shallow environments, and in confined environments such as tanks, the relationships between particle motion and pressure are more complex. Such conditions are met for invertebrates living in nearshore shallow habitats, in close proximity to the seafloor, or in laboratory enclosures. In these environments, particle acceleration cannot be accurately estimated from pressure via the plane wave approximation (Hawkins \& Popper, 2018; Nedelec et al., 2016; Rogers et al., 2016). Rather, it must be directly measured (Gray et al., 2016; Nedelec et al., 2016), such as with an accelerometer (as in Chapters 2-4, measurements in tanks), or estimated via measured pressure differences between pairs of hydrophones (as in Chapter 5, measurements at shallow coral reefs). Further, the direction of particle motion can only be quantified via transducers measuring along multiple axes, and not measurements from single pressure transducers.

Plane wave propagation is limited by depth and frequency. There is a "cutoff frequency" below which sounds will not propagate as plane waves, defined as:

$$
f_{c}=\frac{\left(\pi-\rho_{s e d} / \rho_{w}\right)}{2 \pi \sin \psi_{c}} \frac{c_{w}}{H},(2)
$$


where $\rho_{\text {sed }}$ and $\rho_{w}$ are the densities $\left(\mathrm{kg} \mathrm{m}^{-3}\right)$ of the sediment and seawater, respectively, $c_{w}$ is the sound speed in seawater $\left(\mathrm{m} \mathrm{s}^{-2}\right), \psi_{c}=\arccos \left(c_{w} / c_{s e d}\right)$, where $c_{\text {sed }}$ is the sound speed in the sediment $\left(\mathrm{m} \mathrm{s}^{-1}\right)$, and $H$ is the water depth (m) (Ainslie, 2010). Thus for a given substrate, shallower depths will have greater $f_{c}$.

\subsection{ANTHROPOGENIC NOISE POLLUTION FROM AN INVERTEBRATE PERSPECTIVE}

In the past century, anthropogenic noise has become more prevalent in underwater environments due to increased human use of the oceans. For example, shipping activity and associated noise has increased dramatically over recent decades and is predicted to further increase in this decade (Kaplan \& Solomon, 2016; McDonald et al., 2006). Other common anthropogenic noise sources include sonar from resource exploration, geophysical research, and military operations, noise from construction of marine platforms, and operational noise from marine energy platforms such as wind turbines (Mooney, Andersson, et al., 2020;

Slabbekoorn et al., 2010). Anthropogenic noise dominates low frequencies ( $<10 \mathrm{kHz}$ ), and has peak frequencies often in the hearing range of invertebrates $(<1 \mathrm{kHz})$. Similar to research involving animals' detection of and responses to natural sounds, research of anthropogenic noise impacts on invertebrates is limited. Given the overlap in animals' sound sensitivities and the frequencies of anthropogenic noise, many invertebrates may detect and respond to anthropogenic sounds (Carroll et al., 2017; Murchy et al., 2020; Roberts \& Elliott, 2017).

Studies on invertebrate responses to various anthropogenic noise sources have revealed a suite of behavioral changes, including startle responses of squid (Fewtrell \& McCauley, 2012), hermit crabs (Roberts et al., 2016), and mussels (Roberts et al., 2015b), impaired feeding and resource searching of crabs (Roberts \& Laidre, 2019; Wale et al., 2013a), delayed metamorphosis of pre-settlement crabs (Pine et al., 2012), impaired escape behaviors in rock lobsters (Day et al., 2019), and physiological changes indicating stress or reduced capacity for homeostasis in scallops and crabs (Day et al., 2017; Wale et al., 2013b). Temporary reductions in hearing sensitivity and masking effects (reduced detectability of ecologically relevant cues, in 
part due to lower signal-to-noise ratios) during noise have been demonstrated in some marine mammals and fishes (Caiger et al., 2012; Kastelein et al., 2016; Popper et al., 2005), but have not been shown in invertebrates.

Much of my Thesis (Chapters 2-4) focuses on impacts of pile driving as an anthropogenic noise source, which involves repeated hammering of large, cylindrical piles into the seabed to support foundations of offshore energy platforms such as wind turbines or oil rigs, or nearshore docks, piers, and boat slips. Research characterizing pile driving noise and its impacts on marine life has recently grown due to increased pile driving activity as the offshore wind industry develops globally (Musial et al., 2020). Water-borne pile driving noise exceeds peak sound pressures of $200 \mathrm{~dB}$ re $1 \mu \mathrm{Pa}$ within a few hundred meters from the pile, and is detectable above natural ambient ocean noise as far as $80 \mathrm{~km}$ from the pile (Bailey et al., 2010; Lippert \& von Estorff, 2019; Lippert \& von Estorff, 2014). Sound waves propagate through the seabed as well, but this is rarely measured (Amaral et al., 2018; Reinhall \& Dahl, 2011; Roberts \& Elliott, 2017a). Pile driving schedules for offshore wind farms are variable, depending on platform type, benthic composition, pile dimensions, etc., and several driving bouts may occur per day, each ranging from several minutes to several hours in duration. Driving may span weeks to months depending on project scale (Amaral et al., 2018; Tougaard et al., 2009). Therefore, organisms occupying both the water column and the substrate are exposed to this intermittent but intense noise over great spatial and temporal scales. Pile driving has complex sound propagation, with sound waves radiating from reflected vibrations up and down the pile, and reflections off the seafloor and sea surface outward horizontally from the pile (Reinhall \& Dahl, 2011). Consequently, pile driving noise may not be accurately approximated simply as a plane wave, and the usual distinctions between near field and far field (described above) do not hold (Ainslie et al., 2020), necessitating particle motion measurement in order to understand its influences on marine invertebrates. 


\subsection{CEPHALOPODA AS A MODEL TAXON FOR INVERTEBRATE BIOACOUSTICS}

Cephalopods are mollusks belonging to the class Cephalopoda, including squid, cuttlefishes, and octopuses (and nautiluses, which are not considered here). These animals, especially select species of squid such as Doryteuthis (formerly Loligo) pealeii, common cuttlefish Sepia officinalis, and octopuses such as Octopus vulgarus, have long been the subjects of neurobiological and behavioral research (Gilbert et al., 1990; Hanlon \& Messenger, 2018). For certain cephalopod species, much is known about the behaviors of these enigmatic animals, including behaviors employed for defense, camouflage, feeding, and reproduction, and they are models for ethology and neurobiology (Hanlon \& Messenger, 2018; Hanlon et al., 1999a; Shashar \& Hanlon, 2013). Extensive investigations have been conducted on cephalopod sensory systems, including visual receptors, mechanoreceptors, chemoreceptors, and neural pathways between these sensory systems, the brain, and "effectors", e.g., muscles that carry out behavioral responses to sensory stimuli (Gleadall \& Shashar, 2004; Hanlon \& Messenger, 1988; Hanlon \& Shashar, 2003; Wells \& Wells, 1956). Further, learning and cognitive abilities, many of which are considered "advanced" among invertebrate taxa, have been described in multiple cephalopod species, including habituation of Lolliguncula brevis to visual stimuli (Long et al., 1989), and associative learning, spatial learning, and memory of S. officinalis, O. vulgarus, and other species (Mather, 1991; Mather \& Kuba, 2013; Scatà et al., 2016; Schnell et al., 2021). Several species such as the Hawaiian bobtail squid (Euprymna scolopes) have been cultured as model organisms for studying developmental biology, neurobiology, gene regulation, cospeciation, and various aspects of behavior and physiology (Hanlon et al., 1997; Kerwin et al., 2021; Montague et al., 2021; Zepeda et al., 2017).

The longfin squid D. pealeii, which is the study species for Chapters $2-4$, has been the study subject of decades of neurophysiological and behavioral research. It has long been a model species for biomedical and neurobiological work, and much of the basic knowledge of nerve fiber mechanisms has been obtained from the giant axon of this species (Gilbert et al., 1990). Behaviors of $D$. pealeii have been extensively observed and quantified, including antipredator defense behaviors and strategies (Crook et al., 2014; Staudinger et al., 2011), and 
reproductive behaviors around egg spawning beds (Shashar \& Hanlon, 2013). A detailed ethogram describing their behavioral repertoire has been published (Hanlon et al., 1999).

Compared to other marine invertebrate phyla, the morphology of the cephalopod sound detection sensory organ, the statocyst, is relatively well understood (Budelmann, 1979, 1990; Hanlon \& Budelmann, 1987). However, bioacoustic research on cephalopods is in its infancy; little is known about their sensitivities to sound outside a handful of studies (e.g., Budelmann and Williamson 1994; Mooney et al. 2010; Packard et al. 1990; Samson et al. 2016). There is a clear avenue to leverage the broad wealth of foundational neurophysiological, behavioral, and ecological research on cephalopods toward examining these animals' acoustic sensitivities.

Beyond interest among research groups, cephalopods are of considerable commercial value, making up a $6 \%$ share (USD) of global exports and about $4.5 \%$ of global capture production from 2012-2018 (FAO, 2021). Select species hold great commercial value in certain regions. For instance, the longfin squid (D. pealeii) fishery in New England has had annual landed values of about $\$ 30$ million since 2010 (NMFS, 2021). Cephalopoda is further considered an ecological keystone taxon because many species occupy central positions in marine food webs (Boyle \& Rodhouse, 2005). Cephalopods comprise large portions of the diets of many marine mammals, seabirds, and predatory fish. For example, squid can constitute over $50 \%$ of regional seabird diets and up to $95 \%$ of odontocete diets [ibid]. In turn, many cephalopods are opportunistic predators that feed on a wide variety of prey throughout their lifetime, such as copepods (consumed by pelagic pre-adults), benthic crustaceans and bivalves, and fishes (Boyle \& Rodhouse, 2005; Hunsicker \& Essington, 2006). The widespread ecological roles and the commercial values of cephalopods incentivize their use in research to better understand marine invertebrates' ecological uses of sound and how anthropogenic noise pollution may adversely impact individual fitness and populations. 


\subsection{SOUND DETECTION ABILITIES OF CEPHALOPODS}

Squid, cuttlefish, and octopuses possess paired fluid (endolymph) filled sensory organs called statocysts, which function as gravity and particle motion receptors (Budelmann, 1990, 1992b). The pair of statocysts is located in the head, just ventral and posterior to the eyes. Statocyst morphologies in decapod cephalopods (cuttlefish and squid) indicate these animals should be able to detect particle motion three-dimensionally. The statocysts contain three orthogonally arranged hair cell beds ("maculae"). Each statocyst contains a hard calciumcarbonate (aragonite-based) "statolith", which rests on one of the maculae. The other two maculae have morphologically different but functionally similar structures ("cupulae") overlying them. The statoliths and cupulae are denser than the surrounding fluid and tissue of the squid. In principle, the statocysts operate as mass-loaded, inertial accelerometers.

In an acoustic field, the squid vibrates in phase with the particle motion of the water surrounding it, the squid itself in essence acting as "particle". The statolith and cupulae lag behind in movement and displace underlying hair cells which send neural signals to the brain (Kaifu et al., 2008). Like those of fishes, the hair cells themselves are directionally sensitive, each having a particular axis of maximal sensitivity to motion (Budelmann, 1979; Budelmann \& Williamson, 1994; Sisneros \& Rogers, 2016). Particle acceleration, rather than particle displacement or velocity, is considered the relevant transduction stimulus for statocyst structures of invertebrates, as well as the analogous inner ears of fishes ("otocysts") (Kaifu et al., 2008; Mooney et al., 2010; Packard et al., 1990). Cephalopods sense frequencies below $1000 \mathrm{~Hz}$ and many are most sensitive to those below $100 \mathrm{~Hz}$ (Mooney et al., 2010; Packard et al., 1990; Wilson et al., 2018). Therefore, the hearing capabilities of cephalopods and the physical propagation of sounds favor greater detectability of particle acceleration at relatively low frequencies.

Ecological functions for cephalopods' hearing abilities remain uncertain, though behavioral studies suggest particle acceleration cues may be utilized for detecting and evading predators (Wilson et al., 2018; York \& Bartol, 2014). Additional hypothetical uses include detection of nearby prey (Budelmann et al., 1991), or use of an auditory scene for orientation 
and navigation (Fay, 2009). Cephalopods are not known to produce purposeful sound for communication (Hanlon \& Messenger, 2018).

Several previous studies have observed structural damage to cephalopods' sensory organs after exposure to anthropogenic noise (Solé et al., 2013, 2017), and behavioral alarm and escape responses to air gun noise or artificial tones and vibrations (Fewtrell and McCauley 2012; Mooney et al. 2016; Samson et al. 2014; Wilson et al. 2018). Overall, cephalopods appear vulnerable to physical and behavioral effects of anthropogenic noise. Due to the high ecological and commercial importance of cephalopods, the large existing body of baseline neuroethological research on them, and growing examples of cephalopods' responses to sound, Cephalopoda is a suitable model taxon for investigating sound detection by, and effects of anthropogenic noise on marine invertebrates.

\subsection{CORAL REEFS AS MODEL ECOSYSTEMS FOR MARINE SOUNDSCAPE STUDIES}

Coral reef structure provides habitat for diverse and abundant invertebrate and fish communities, including many species that produce sounds (Lobel et al., 2010; Mooney, lorio, et al., 2020; Tricas \& Boyle, 2014). Coral reefs are increasingly threatened by a multitude of human-driven factors including terrestrial runoff, noise pollution, hypoxia, ocean acidification, and warming (Altieri et al., 2017; Fabricius, 2005; Ferrier-Pagès et al., 2021; Hughes et al., 2018; Mollica et al., 2018). Caribbean reefs have further suffered substantial losses in live coral cover from a recent outbreak of a novel stony coral tissue loss disease (Heres et al., 2021). Given the strong environmental stressors currently faced by coral reefs and their rapidly changing community structures due to these stressors (Tsounis \& Edmunds, 2017), there is critical need among research, regulatory, and conservation bodies to develop and apply efficient and practical methods for monitoring and predicting these changes, and for assessing overall reef health.

"Ecoacoustics", or the study of natural soundscapes and their connections to acoustic ecology, is an emerging and rapidly growing research field (Farina \& Gage, 2017). Among 
underwater soundscapes, those of coral reefs have received substantial attention due to their complexity and the diversity of soniferous (sound-producing) coral reef inhabitants (Lobel et al., 2010; Tricas \& Boyle, 2014), and growing interest in how coral reef sounds are utilized by preadult fishes and invertebrates as settlement cues (Gordon et al., 2018; Lillis, Apprill, et al., 2018; Suca et al., 2020). There is strong motivation to apply soundscape data to monitor and predict changes in community structure of reefs and other underwater habitats, for example by finding links between traditional non-acoustic indicators of reef health and acoustic characteristics of their soundscapes, or even using reef sounds to encourage fish settlement as part of reef restoration (Gordon et al., 2019; Mooney, lorio, et al., 2020).

To date, these studies have favored reporting acoustic sound pressure measurements. Those that address particle motion focus on brief "snapshot" recordings lasting minutes to days (Jesus et al., 2020; Kaplan \& Mooney, 2016). There are no studies that quantify particle motion cues of these soundscapes over longer periods, address the natural temporal variability in cues available, or compare particle motion levels of these cues among reefs.

This lack of particle motion data leaves a major gap in our understanding of acoustic ecology and the auditory scene for multiple reasons. For one, hearing is a fundamental sensory modality. We know of no deaf taxa. Second, particle motion is the primary acoustic stimulus for most fishes and invertebrates. Third, we know many underwater soundscapes have substantial variability in sound pressure, and it is unclear whether particle motion is similarly variable. This variability may be temporal, e.g., over diel, lunar and seasonal periods (Kaplan et al., 2018; Staaterman et al., 2014), and spatial, e.g., between reefs of different coral cover and fish abundance (Kaplan et al., 2015; Lillis, Caruso, et al., 2018; Radford et al., 2014). Outlier pressure levels of cues such as fish sounds or snapping shrimp snaps also have substantial variability in amplitude above median ambient levels (Kaplan \& Mooney, 2016; Salas et al., 2018). Further, as noted earlier, for many shallow reef environments particle acceleration will not scale predictably (as for a plane wave) with sound pressure. We cannot predict how particle motion cues will vary and impact reef biota from our pressure measurements. Thus we are completely unaware of the availability of this cue for the animals that depend on reefs, and it is imperative 
that more coral reef soundscape studies report particle motion to place available soundscape cues in the context of what the majority of sound-sensitive coral reef animals detect.

\subsection{THESIS OVERVIEW}

My Thesis aims to improve understanding of invertebrate behavioral responses to anthropogenic noise and natural particle motion cues of soundscapes detectable by marine invertebrates, leveraging squid as model organisms and coral reefs as model habitats.

Chapters 2-4 are unified under a common theme of quantifying behavioral responses of the longfin squid, Doryteuthis pealeii, to laboratory exposures of pile driving noise. Noise played to squid was originally recorded from the construction of an offshore wind farm near Block Island, Rhode Island. A primary impetus of this project was the growing concern among ocean energy management entities and fishing industry members over how expansion of offshore wind energy (specifically in Northeast U.S. continental shelf waters) could negatively impact commercially and ecologically valuable species, including D. pealeii. In addition to being highly relevant to marine resource management, this project offered an opportunity to study behavioral responses to anthropogenic noise in great detail and in multiple contexts. These studies lay the groundwork for continued investigation of cephalopods' sensitivities to anthropogenic stressors. These chapters span three different behavioral contexts, including individual squid swimming at rest, individual squid involved in feeding tasks, and groups of squid involved in reproductive behaviors.

In Chapter 2, "Impulsive pile driving noise elicits alarm responses in squid (Doryteuthis pealeii)," I reported multiple types of squid alarm behaviors (naturally employed for predator defense and evasion) elicited by noise, over repeated impulses and exposure days. I conducted these experiments in a controlled tank environment and calibrated the particle motion and sound pressure fields in the tank with high spatial resolution. I investigated habituation (learned decreases in response over time) to noise exposure and amplitude-dependent responses to establish baseline dose-response patterns to pile driving noise. I then discussed potential implications of these observations for squids' responses to predators. 
In Chapter 3, "Changes in feeding behavior of longfin squid (Doryteuthis pealeii) during laboratory exposure to pile driving noise," I examined squids' predation behavior on killifish (Fundulus heteroclitus) prey. I monitored squid alarm behaviors and killifish locomotion when noise commenced during active feeding, and when squid were allowed to feed minutes after noise began. This study used a similar experimental setup to Chapter 2. I quantified prey capture rates, time taken to capture, and missed predation attempts. I also conducted novel hearing measurements on killifish to determine their ability to detect the noise treatment. I discussed ecological implications of squids' altered feeding behavior during noise.

In Chapter 4, "Context is key: squid reproductive behaviors and spawning withstand wind farm pile driving noise," I reported the reproductive dynamics of mixed-sex groups of squid in a similar but larger laboratory setup compared to that of the previous two chapters. I quantified mating, egg laying, agonistic (fighting) behaviors and other key mating-related behaviors over three successive bouts of pile driving noise. I compared results with those from Chapters 2 and 3 to discuss context-dependence of noise effects on $D$. pealeii; a theme that has been widely discussed for the study and management of marine mammals (Ellison et al., 2012), but not for marine invertebrates with respect to anthropogenic noise impacts.

In Chapter 5, "Natural cues for invertebrate and fish hearing: particle motion measurements on reefs," I reported multiple months of particle acceleration data recorded from coral reefs of varying habitat quality, and compared particle motion trends with those of pressure data. I then placed these data in the context of known particle acceleration sensitivities of marine invertebrates and fishes to address the acoustic cues available on reefs. Though the primary focus of my Thesis is on invertebrate taxa, I also considered fishes in this chapter because they detect particle motion, and have similar frequency ranges of hearing compared to marine invertebrates $(<1 \mathrm{kHz})$. Further, predominant biological coral reef sounds in this frequency range are produced by fishes, including many for communication, e.g., courtship and predator defense (Tricas \& Boyle, 2014). These fish sounds further may be utilized by invertebrates as navigation or settlement cues (Lillis, Apprill, et al., 2018; Salas et al., 2018; Vermeij et al., 2010) or for detecting the presence of predators (Hughes et al., 2014). 
Appendices A-D include supplementary results and methods for Chapters $2-5$. In Appendix E I provide further detail and recommendations on the measurement and reporting of underwater sound fields in tanks, utilizing recordings from the tank setup in Chapters 2 and 3. In Appendix F I provide example MATLAB scripts I wrote to calculate particle acceleration throughout my Thesis.

In Chapter 6, "Conclusions and future directions," I synthesize results from Chapters 2-5 to discuss how my research has contributed to broader understanding of cephalopods' and invertebrates' sensitivities to anthropogenic noise, and the relevance of natural particle motion cues to invertebrates. I consider implications of squids' context-dependent responses to anthropogenic noise from ecological, offshore energy development, and noise pollution management perspectives. I place my conclusions from the coral reef soundscape study in broader contexts, including the utility of these methods for answering questions about invertebrates' and fishes' ecological use of particle motion, and the applications of soundscape data to habitat monitoring and conservation. As well, I make recommendations on different methods of particle motion measurement and instrumentation based on my experiences throughout my Thesis, and outline the pros and cons of these methods for different research applications. Finally, I describe remaining knowledge gaps and recommended foci for future investigation of invertebrates' use of natural sounds and effects of anthropogenic noise on invertebrates. 


\section{CHAPTER 2 : IMPULSIVE PILE DRIVING NOISE ELICITS ALARM RESPONSES IN SQUID (DORYTEUTHIS PEALEII)}

This chapter was originally published as: Jones, I. T., Stanley, J. A., Mooney, T. A. (2020). Impulsive pile driving noise elicits alarm responses in squid (Doryteuthis pealeii). Marine Pollution Bulletin, 150. 110792. https://doi.org/10.1016/i.marpolbul.2019.110792. The Supplementary Materials for this chapter can be found in Appendix A. 


\begin{abstract}
Pile driving occurs during construction of marine platforms, including offshore windfarms, producing intense sounds that can adversely affect marine animals. We quantified how a commercially and economically important squid (Doryteuthis pealeii: Lesueur 1821) responded to pile driving sounds recorded from a windfarm installation within this species' habitat. Fifteen-minute portions of these sounds were played to 16 individual squid. A subset of animals $(n=11)$ received a second exposure after a $24-h$ rest period. Body pattern changes, inking, jetting, and startle responses were observed and nearly all squid exhibited at least one response. These responses occurred primarily during the first 8 impulses and diminished quickly, indicating potential rapid, short-term habituation. Similar response rates were seen 24$\mathrm{h}$ later, suggesting squid re-sensitized to the noise. Increased tolerance of anti-predatory alarm responses may alter squids' ability to deter and evade predators. Noise exposure may also disrupt normal intraspecific communication and ecologically relevant responses to sound.
\end{abstract}




\section{INTRODUCTION}

Sound travels efficiently in the ocean, often over long distances, and is a readily available sensory cue for many marine animals. Sound cues are utilized by marine animals for many ecologically important functions, including predator and prey detection, navigation, and communication with conspecifics. However, anthropogenic ocean noise can impair soundsensitive animals' ability to detect vital sound cues, and can have detrimental physiological, behavioral, and ecological impacts on marine animals (Gedamke et al., 2016; NRC, 2003). Predominant sources of marine anthropogenic noise include vessel traffic, seismic surveys, and marine construction activity (NRC, 2003). Research regarding anthropogenic ocean noise impacts and noise regulations have focused mainly on marine mammals and fish, and far less is known regarding impacts on aquatic invertebrates (Gedamke et al., 2016; Hawkins et al., 2015).

Impulsive pile driving is a major noise source of concern (Bailey et al., 2014; Dahl et al., 2015). This sound is associated with the construction of docks, piers, and offshore energy platforms (e.g., for wind turbines), propagating from hammering piles (large pipes or poles) into the seabed. Offshore pile driving activity is increasing in many waters around the globe due to a rapid expansion of offshore wind energy (Bailey et al., 2014; Musial et al., 2020). The U.S. is in the early stages of offshore wind development with only one operational offshore wind farm as of 2019, located southeast of Block Island, Rhode Island. However, with 28 additional offshore wind farm projects in the development pipeline (Musial et al., 2020), the occurrence rate and spatial range of pile driving noise are likely to increase in U.S. waters in the near future.

Offshore pile driving noise is typically high-amplitude at its source, and depending on pile characteristics and environmental conditions, also tends to propagate great distances though the water and seabed, and is often detectable in the water column above ambient ocean noise levels over $10 \mathrm{~km}$ away (Bailey et al., 2010; Madsen et al., 2006). This noise may have a wide variety of adverse impacts on marine animals, although most research has focused on short-term (e.g., within a day) rather than longer term effects. Physiological impacts in vertebrate taxa may include temporary reductions in hearing sensitivity, as shown in harbor porpoises (Kastelein et al., 2016), damage to bodily tissues, as shown in some fish species (Casper, Halvorsen, et al., 2013; Halvorsen et al., 2011), and changes in oxygen consumption in 
fish (Bruintjes et al., 2017; Debusschere et al., 2014). Locomotor alarm responses are also often observed in fish at the onset of pile driving noise (Kastelein et al., 2017; Spiga et al., 2017). Such responses are associated with the perception of a predatory threat and may indicate general anxiety and stress (Maximino et al., 2010). Several studies have addressed simulated pile driving noise impacts on benthic invertebrates such as bivalves and crustaceans. Substrateborne vibrations generated in a tank induced apparent alarm responses in hermit crabs (Pagurus bernhardus), inducing sudden onset or cessation of locomotion, and digging (Roberts et al., 2016). In a similar experimental setup with the same vibration stimuli, blue mussels (Mytilus edulis) reduced valve gape, sometimes to full closure, potentially disrupting normal feeding, excretion, and respiration (Roberts et al., 2015). During pile driving in situ, M. edulis increased clearance rates, suggesting an increased metabolism as a stress response (Spiga et al., 2016).

The longfin squid, Doryteuthis pealeii, (Lesueur 1821; formerly Loligo pealeii) is a commercially important species in the United States, contributing mean annual landings and value of about 11,000 mt and $\$ 30$ million since 2010 (NMFS, 2021). These squid are considered ecologically important in marine food webs, since they are prey items for many marine mammals, seabirds, and fish, and are predators of many small fish and crustaceans (Boyle \& Rodhouse, 2005; Hunsicker \& Essington, 2006). Doryteuthis pealeii is relatively widespread, inhabiting coastal waters in the western Atlantic from Newfoundland to Venezuela, and has a typical lifespan of 9-12 months (Hanlon et al., 2013). This species is most abundant between Georges Bank and Cape Hatteras, NC (Hanlon et al., 2013). Within its range, lease and call areas for future offshore wind farm construction currently exist in 9 U.S. states (Musial et al., 2020). This species is considered epi-benthic: wild adult $D$. pealeii generally forage near and rest on the seabed during daylight and disperse higher in the water column at night (Hanlon et al., 2013). Given the overlapping range of these squid and offshore windfarm construction, it is expected that this taxon will face both water-borne and seabed-borne pile driving sounds.

Longfin squid detect water-borne sounds, and potentially detect substrate-borne sounds as well (Mooney et al., 2010; Roberts \& Elliott, 2017). They exhibit a suite of behavioral alarm responses to water-borne pure tone sounds at and below frequencies of $1 \mathrm{kHz}$, with 
occurrence rate and intensity of responses dependent on stimulus amplitude (Mooney, Samson, et al., 2016). Studies of behavioral and physiological hearing thresholds indicate this species is most sensitive to sounds between $100-300 \mathrm{~Hz}$; physiological hearing thresholds in this range are about -26 dB re $1 \mathrm{~m} \mathrm{~s}^{-2}$ (Mooney et al., 2010; Mooney, Samson, et al., 2016). Importantly, sound energy of water-borne pile driving noise is typically highest within this same range (Amaral et al., 2018; Nedwell et al., 2007). Squid appear to only sense acoustic particle motion (the back-and-forth vibratory component of sound), with particle acceleration likely being the most relevant metric (Budelmann, 1990; Packard et al., 1990). There is no evidence to-date for sound pressure detection by cephalopods. Cephalopods detect particle acceleration via paired statocyst organs in the head, which contain a calcium-carbonate 'statolith' sensitive to linear acceleration; the statolith is denser than the surrounding tissues and lags relative the rest of the animal when set in motion by acoustic/vibratory stimuli, and displaces underlying hair cells to transduce this motion to a neural signal (Budelmann, 1990).

The ecological functions of squid and other cephalopods' hearing abilities are unknown. Cephalopods might utilize sound to assess the 'auditory scene' of their natural environment, orienting to and extracting information from their environment by segregating discrete components of natural soundscapes, which is thought to be a basal function of hearing (Fay, 2009). Sound might also be utilized by squid to detect the presence of nearby predators (York \& Bartol, 2014), especially when vision is impeded, e.g., at night or in murky water. Squid are not known to utilize sound for communication (Hanlon \& Messenger, 2018). Indeed, their primary communication system is considered to be visually based, leveraging complex chromatophore skin patterning (Hanlon \& Messenger, 2018). Vibrant and subtle colorations and pattern grades can indicate a range of signals displayed to predators and conspecifics, and squid depend on such displays for survival and reproduction. Some of these pattern changes serve as 'alarm' signals to bluff or intimidate predators, some serve to camouflage squid in order to avoid detection by predators, and others are signals displayed to conspecifics during courtship and agonistic (fighting) sequences when competing for mates. Other behaviors such as releasing ink and escape jetting are important for defense against predators. Survival and reproduction could 
be influenced across sensory modalities if changes in such visual communication and locomotor defense behaviors occur during noise exposure.

Regardless of natural function of their sound-sensitivity, squid and other cephalopods may be vulnerable to adverse behavioral and physiological effects of anthropogenic noise. Laboratory studies utilizing ferry noise elicited apparent behavioral stress responses in cuttlefish (Sepia officinalis) including increases in frequency of visual displays and time spent swimming (Kunc et al., 2014). In situ exposure of caged squid (Sepioteuthis australis) to impulsive noise from air guns induced behavioral alarm responses such as jetting (Fewtrell \& McCauley, 2012). Though results from this small handful of studies suggest adverse effects, noise sources and cephalopod species are diverse, and little is known regarding how $D$. pealeii or other cephalopod species may behaviorally respond to anthropogenic noise.

This study sought to determine how $D$. pealeii individuals behaviorally respond to waterborne pile driving noise. To limit extraneous stimuli, experiments were conducted in a relatively quiet, controlled laboratory environment. Playback stimuli were pile driving impulse sounds recorded during construction of the Block Island Wind Farm (BIWF) in Rhode Island. Our goal was to categorize the types of responses demonstrated and their occurrence rate with respect to noise duration and received sound level in a controlled, consistent and well-calibrated environment. Response rates were first quantified during initial (15 $\mathrm{min}$ ) noise exposures to pile driving noise. Exposures were repeated after a 24-h rest period to evaluate potential long-term habituation. Both particle motion and sound pressure were quantified in order to describe received levels of the sound component relevant to squid (particle motion) and to support the use of these data for potential management needs.

\section{METHODS}

\section{Animal collection and holding conditions}

Squid were collected in Vineyard Sound via trawl, by the Marine Biological Laboratory (Woods Hole, MA). As the collection vessel returned to dock, squid with minimal physical damage (e.g., few or no skin lesions or tears) were gently hand-transferred to coolers filled with 
ambient temperature Vineyard Sound seawater. The squid were immediately driven to the Environmental Systems Laboratory (ESL) at Woods Hole Oceanographic Institution, Woods Hole MA, where husbandry and experiments were conducted. Upon arrival at the ESL, squid were immediately and gently hand-transferred into three circular holding tanks of at least $1.2 \mathrm{~m}$ diameter $(3400 \mathrm{~L})$, with ambient flowing seawater and air stones to ensure high dissolved oxygen (DO). All tanks were located in a facility subject to a natural light cycle. Sexes were kept separate and densities were below one squid per $680 \mathrm{~L}$. During the experimental period, means \pm SD of environmental measurements across holding tanks were as follows: temperature: 20.63 $\pm 1.06{ }^{\circ} \mathrm{C}$; salinity: $30.49 \pm 1.40 \mathrm{PSU} ; \mathrm{pH}: 7.93 \pm 0.05$; DO saturation: $100 \pm 2 \%$. Squid were handfed daily with Fundulus spp. collected from local estuaries (WHOI IACUC approval to TAM). Squid were held in these lab conditions for a minimum of 24 hours prior to being tested, and experimenters took care to minimize sound in and near the holding tanks during this period.

\section{Experimental tank and playback setup}

Experiments took place in 2017 from September $2^{\text {nd }}$ to October $28^{\text {th }}$ ( $n=23$ trials), and in 2018 from June $15^{\text {th }}$ to June $19^{\text {th }}$ ( $\mathrm{n}=11$ trials). All experiments were conducted in a $110 \mathrm{~cm}$ diameter circular tank filled to a $50 \mathrm{~cm}$ depth with ambient flowing seawater (Fig. 1). The tank was isolated from nearby vibrations with cinderblocks, plywood, and two layers of neoprene between the tank and the concrete floor of the lab. An UW-30 underwater speaker (ElectroVoice, Fairport, NY) was suspended, facing horizontally, $23 \mathrm{~cm}$ from the surface and $15 \mathrm{~cm}$ from the closest tank wall. To ensure squid did not swim into or behind the speaker, a plastic mesh barrier attached to a PVC frame was placed $15 \mathrm{~cm}$ in front of the speaker. To monitor ambient tank sound and noise playbacks during experiments a hydrophone (High-Tech Inc., Long Beach, MS) was placed just behind the mesh barrier $1 \mathrm{~cm}$ from the tank wall, $44 \mathrm{~cm}$ from the speaker, and $35 \mathrm{~cm}$ deep. The hydrophone was attached to a Song Meter SM2 (Wildlife Acoustics, Maynard, MA) data acquisition device. Two cameras were used to record squid behavioral responses to pile diving stimuli. An overhead camera (HDRCX440 Handycam, Sony) was used for all quantitative video analyses and a GoPro mounted above and to the side of the tank allowed a closer view of subtle behaviors post-hoc. Two $15 \mathrm{~cm}$ long rulers were placed on the top and 
bottom of the tank to determine depth of squid in video analyses. A red LED light was clamped to the side of the tank in view of the camera but not visible to the squid, to indicate in subsequent video analyses when pile noise or control playbacks were on.

Audio files of pile driving noise used for experiments were recorded during construction of BIWF on October 25 th 2015 (between 16:00 and 20:00 UTC), from a hydrophone (High Tech Inc., model: HTI-94-SSQ, sensitivity: $-203.8 \mathrm{~dB}$ re $1 \mathrm{~V} / \mu \mathrm{Pa}$, gain: $6 \mathrm{~dB}$, flat frequency response from $2 \mathrm{~Hz}$ to $30 \mathrm{kHz}$ ) on a benthic sled located $26 \mathrm{~m}$ deep and $0.5 \mathrm{~km}$ away from a pile driving site (Amaral et al., 2018). The hydrophone was about $1 \mathrm{~m}$ above the seabed, and part of a tetrahedral array of hydrophones used to calculate particle acceleration via pressure differentials between hydrophones along three orthogonal axes. The hydrophones were spaced $0.5 \mathrm{~m}$ apart, and were the same model and had the same sensitivity and frequency response noted above. These acceleration data, in $\mathrm{m} / \mathrm{s}^{2}$, were provided to the authors (see acknowledgements). These files were recorded at a $9766 \mathrm{~Hz}$ sample rate. The steel, hollow pile had a diameter of $127.0 \mathrm{~cm}$, wall thickness of $3.8 \mathrm{~cm}$, a rake of $13.27^{\circ}$ with respect to vertical, and was driven up to $76.2 \mathrm{~m}$ deep into the seabed.

To prevent pseudoreplication of playback stimuli, three 15 min long recordings, hereafter referred to as Pile1, Pile2, and Pile3, were extracted from two different pile driving bouts and edited in Adobe Audition (Adobe Systems, San Jose, CA) prior to playback. A 3-s fadein of the sound file (before the pile driving sound was emitted) was applied to each recording to prevent artifacts that could result from the playback beginning at a higher amplitude. Each recording was amplified by a custom magnitude to obtain the highest playback sound levels possible without clipping, with the goal to match received zero-to-peak sound pressure levels in the tank with those present $0.5 \mathrm{~km}$ from the BIWF pile driving site, i.e. 190-194 dB re $1 \mu \mathrm{Pa}$. For Pile1, Pile2, and Pile3 respectively, median inter-pulse intervals were $1.53 \mathrm{~s}$ (IQR: 1.52-1.55 s), $1.81 \mathrm{~s}$ (IQR: 1.80-1.82 s) and $2.35 \mathrm{~s}$ (IQR: 2.32-2.42 s), median zero-to-peak pulse amplitudes were 190.6 dB (IQR: 190.0-191.2 dB), 193.8 dB (IQR: 193.6-194.0 dB), and 194.2 dB (IQR: 193.6-194.5 dB) re $1 \mu \mathrm{Pa}$, and median root-mean-square inter-pulse amplitudes were $140.7 \mathrm{~dB}$ (IQR: 140.5-141.0 dB), 138.4 dB (IQR: 138.2-138.7 dB), and $137.8 \mathrm{~dB}$ (IQR: 137.6-138.0 dB) re 1 $\mu \mathrm{Pa}$. Notably, pulse amplitude, inter-pulse time interval, and inter-pulse interval amplitude 
within a pile driving event will vary slightly from impact to impact and as the pile is driven into the sediment. Here, playback amplitudes and inter-pulse interval were not manipulated to be identical within or across the three pile driving files because we were interested in studying behavioral impacts of pile driving noise at a simulated distance from the pile, rather than studying responses dependent on these specific metrics. The variability in these metrics across impulses reflects that which a wild squid may experience near an offshore pile driving site. In control trials a 15 min long silent file was played.

\section{Experimental procedure}

All trials were conducted during the daytime, and squid were tested individually for each trial (no pairs or groups of squid were tested in a given trial). Trial order for experimentally naïve squid was randomized between controls and the three pile driving noise files. Noise files for retests were also randomly selected regardless of the file used for a squid's first noise exposure. Individual squid (mean mantle length \pm SD: $16.5 \pm 4.1 \mathrm{~cm}, \mathrm{n}=23$ ) were transferred by net into a container with a volume of at least $19 \mathrm{~L}$ for transport to the experimental tank. Only squid that displayed normal behaviors (i.e., normal body patterning, swimming normally in the water column) and were without major skin damage (Hanlon \& Messenger, 2018) were used for experiments. Squid were then hand-transferred into the experimental tank, and hydrophone and video recordings began immediately thereafter. To allow squid to acclimate to the new tank and be able to compare their behaviors before and during noise, squid were allowed 5 min to acclimate in the tank, followed by an additional 15 min (termed the "preexposure" period) before control or pile driving files were played. Playback of pile driving or silent control files ran for $15 \mathrm{~min}$ (the "exposure" period). Trials for which pile driving was played to experimentally naïve squid are hereafter referred to as "Day 1 " trials. To examine the effects of repeated exposures, a subsample of squid was retested $24 \mathrm{~h}$ after the first exposure ("Day 2" trials). To maintain these animals in between trials, they were housed in a separate building in separate, $1.2 \mathrm{~m}$ diameter tanks with opaque covers, but otherwise with the same conditions as pre-experiment holding tanks. The experimental procedure on Day 2 trials was identical to that for Day 1 trials. Sample sizes for each trial type are summarized in Table 1. 


\section{Acoustic calibration of the experimental tank}

The experimental tank was calibrated in $10 \mathrm{~cm}$ increments in all 3 dimensions (224 positions total) without animals present, creating a 3D array of received sound levels. At each position, the first minute of each of the three pile driving noise files was played and recorded at a $48 \mathrm{kHz}$ sampling rate by a triaxial ICP accelerometer (Model W356B11, PCB Piezotronics) and Reson TC4013 hydrophone (Teledyne Marine) spaced $10 \mathrm{~cm}$ apart. The accelerometer was wired through a signal conditioner (Model 480B21, Piezotronics). The accelerometer signal and hydrophone were input to two analog filters (Model 3382, Krohn-Hite Corporation), which each applied an anti-aliasing low-pass filter at $24 \mathrm{kHz}$ and a $20 \mathrm{~dB}$ gain. Output of the filters was input to a data-acquisition board (USB 6251, National Instruments), which was in turn connected to a Lenovo X1 Carbon laptop that ran a custom MATLAB (Mathworks, Natick, MA) script to record the audio files.

\section{Data analysis}

All acoustic data were processed with custom MATLAB scripts. Audio data were first bandpass filtered to $20-1000 \mathrm{~Hz}$ using an $8^{\text {th }}$ order Butterworth filter. This frequency range spans the hearing range of squid and the predominant acoustic energy range of pile driving strikes (Amaral et al., 2018; Mooney et al., 2010; Nedwell et al., 2007). For simplicity, the Euclidean norm of the three measured directions of particle acceleration was calculated (for comparison, the Euclidian norm of particle acceleration of BIWF pile driving recorded in the field was calculated as well). Zero-to-peak sound pressure levels (SPLz-pk) and zero-to-peak sound acceleration levels ( $S A L_{z-p k}$ ) for individual pile pulses were calculated over a time window from $0.15 \mathrm{~s}$ before to $1 \mathrm{~s}$ after the time point of the detected pulse peak. $S P L_{z-p k}$ and $S A L_{z-p k}$ were calculated as:

$$
\mathrm{SPL}_{\mathrm{z}-\mathrm{pk}} \text { or } \mathrm{SAL}_{\mathrm{z}-\mathrm{pk}}=20 * \log _{10}\left(X_{\text {peak }}\right) \text {, (3) }
$$

where $X_{\text {peak }}$ is the maximum absolute $\mu \mathrm{Pa}$ or $\mathrm{m} \mathrm{s}^{-2}$ value over a given measurement period, for pressure and acceleration respectively. Units are $\mathrm{dB}$ re $1 \mu \mathrm{Pa}$ and $\mathrm{dB}$ re $1 \mathrm{~m} \mathrm{~s}^{-2}$ for $S P L_{z-p k}$ and $\mathrm{SAL}_{z-p k}$, respectively. To quantify sound energy distribution over frequencies from $20-1000 \mathrm{~Hz}$, power spectral density (PSD) curves were calculated in $10 \mathrm{~Hz}$ bins for both sound pressure and 
acceleration using Welch's method, with 50\% overlap of time windows. PSD was integrated over pulse windows, defined as the time ranging from $5 \%$ to $95 \%$ of the cumulative curve of time-integrated squared acceleration (or pressure) within the time range of $0.15 \mathrm{~s}$ before to $1 \mathrm{~s}$ after the detected pulse peak. PSD was also calculated over inter-pulse windows, defined as times outside of the pulse windows. In MATLAB, A Kruskal-Wallis test was performed to compare median $S A L_{z-p k}$ levels over the 224 recording positions and among the three pile driving files. Wilcoxon rank sum tests were then used to test pairwise differences between the three files, applying the Bonferroni correction for multiple comparisons, thus testing at a significance level of $\alpha=0.017$. From the acceleration data, particle velocity in $\mathrm{m} \mathrm{s}^{-1}$ was calculated by integrating the time series of acceleration for each axis in frequency space using omega arithmetic, and the Euclidean norm of velocity was calculated.

The squids' in-tank position was quantified using the software Tracker (Open Source Physics) to address behaviors respective to received sound levels during a particular pulse. For the first minute of noise playback, squid positions were measured at the video frame closest in time to the onset of each pile driving pulse. The squid's location in the $X$ and $Y$ plane (see Fig. 1) was found by measuring the perpendicular distances from the center of the squid's head to the $X$ and $Y$ axes, with the center-front edge of the speaker set as the origin. The center of the squid's head is approximately where the statocyst sensory structures involved in particle motion detection are located. Squid depth was calculated based on the lengths of the calibration rulers, known depth difference between them, and the squid's mantle length. For a given playback file and pile driving pulse number, $S P L_{z-p k}$ and $S A L_{z-p k}$ at the calibration position closest to the squid's measured position were taken as the received levels. $S P L_{z-p k}$ of the original playback files varied from pulse to pulse (range of $4 \mathrm{~dB}, 1 \mathrm{~dB}$, and $4 \mathrm{~dB}$ for Pile1, Pile2, and Pile3 respectively over the first minute of playback). Therefore, received levels of each pulse were 'normalized' by their relative amplitudes. This normalization was calculated separately for Pile1, Pile2, and Pile3, with the following equation:

$$
R L_{n o r m, i}=R L_{i}-\left(L_{i}-\operatorname{mean}(L)\right) \text {, (4) }
$$

where $R L_{\text {norm, } i}$ is the normalized received level for impulse $i, R L_{i}$ is the estimated received level, in $\mathrm{dB}$ re $1 \mathrm{~m} \mathrm{~s}^{-2}$, from tank calibration data for a squid at a given tank location during a given 
impulse, $L_{i}$ is the $S P L_{z-p k}$, in dB re $1 \mu \mathrm{Pa}$, of the original playback file for that impulse, and mean $(L)$ is the mean $S P L_{z-p k}$ over the first minute of the original playback file. The field recording used for playback was recorded with a hydrophone, and acceleration data at this location was not available at the time of writing.

To test for trends in normalized received level over the first minute of noise playback, slopes of linear regression lines were found for each individual trial, and differences between slopes were tested with a two-way ANOVA. There were no significant differences in slopes between the 3 pile files $\left(\mathrm{SAL}_{z-\mathrm{pk}}: F=0.28, P=0.76\right)$ or between Day 1 and Day 2 exposures $\left(\mathrm{SAL}_{z-}\right.$ pk: $F=0.19, P=0.67)$; thus all experimental trials were combined and a single linear regression was performed. Spearman's rank correlation was performed to test for a significant trend of received level over the first minute. Median normalized levels were also compared between the first three pile impulses with Mann-Whitney $U$ tests, at a significance level of $\alpha=0.017$ applying the Bonferroni correction for three pair-wise comparisons.

For video analyses, data were subsampled to the last minute of the pre-exposure period, and data from experimental trials were subsampled to sets of 30 pile impulses encompassing the $1^{\text {st }}, 5^{\text {th }}, 10^{\text {th }}$, and $15^{\text {th }}$ minute of the exposure period. Upon initial analysis, behavioral alarm responses were typically timed closely with pile driving pulses (within $1 \mathrm{~s}$ ), thus for noise exposure periods, time windows were binned between the onset of a pile pulse up until the onset of the following pulse. For all other time periods, as well as 'exposure' periods of control trials, behaviors were recorded in bins of $2 \mathrm{~s}$, equal to the average inter-pulse interval of the pile driving noise (1.85 s) rounded to the nearest second. Videos were viewed by a trained observer at half-speed to quantify occurrences of inking, jetting, startle, and body pattern change responses following descriptions from previous studies (Hanlon et al., 1999; Mooney, Samson, et al., 2016). Examples of these behaviors are shown in Figure 2. Body patterns in cephalopods by definition contain chromatic, postural, and locomotor components (Hanlon et al., 1999), but "body pattern changes" reported in the present study refer specifically to changes in chromatic components. These chromatic components arise from changes in skin color and pattern due to a change in the expression of specialized skin cells (chromatophores and leucophores). Following Hanlon et al. (1999), chromatic components 
were classified into "All dark", "Clear" (both indicative of a "major" state of alarm), "White arms", "Dark arms", "Dark arms/head", "Dark arm stripes" (these four indicating a "minor" state of alarm), and "Bands", which typically indicates an attempt at crypsis (camouflage) and serves to hide squid from predators when squid are above or on a natural sandy substrate. These body pattern changes occur naturally during predator encounters and in reproductive contexts (e.g., displays to agonistic males or to mates). In the present study all observed behaviors were recorded, thus more than one response (i.e., inking, jetting, startle, and body pattern change) could be noted at a given time point. For example, inking was always followed by jetting, but jetting could occur in the absence of inking. Recorded body pattern changes were limited to acute patterns (lasting up to several seconds) likely associated with an alarm response. To reduce inclusion of false-positive, extraneous responses, criteria for identifying a body pattern change as alarm-type were that it did not occur: 1) in synchrony with the squid swimming into a tank wall or barrier, 2) as a regular, periodic change timed with swimming movements, or 3 ) as a result of the squid transitioning between sitting on the bottom of the tank and swimming, or vice versa. Notably, Bands often occurred during a transition from bottom-sitting to swimming, but any Bands reported here occurred only when the squid was swimming.

Fisher's exact tests were performed to compare proportions of behaviors between Day 1 and Day 2 trials, within given impulse bins, or within and between longer experimental time periods. When comparing behaviors among Day 1, Day 2, and controls, or among three time periods, the Bonferroni correction was used, yielding a significance level of $\alpha=0.017$. Generalized linear models (GLMs) were used for regression analysis in $\mathrm{R}$ to describe the occurrence of each behavior over the first 30 pile impulses (R Core Team, 2017). Two GLM models were used for each of the four behavior types: the first modeled Day 1 and Day 2 on separate curves with pile impulse number as the only explanatory variable, and the second compared the effects of both pile impulse number and treatment day (Day 1 or Day 2) for data from both days. Initially, zero-inflated Poisson GLMs ran using the 'pscl' package (Zeileis et al., 2008) indicated no significant excess zeros in the data. Thus, an ordinary Poisson, log-link GLM was performed. Subsequently, Nagelkerke/Cragg-Uhler pseudo $\mathrm{R}^{2}$ values were calculated using 
the "rcompanion" package (Mangiafico, 2017). Although these pseudo $\mathrm{R}^{2}$ values have the same range (i.e. from 0 to 1 ) as $R^{2}$ values from ordinary least squares (OLS) regression, they are not directly comparable to $\mathrm{R}^{2}$ values from OLS models and cannot be interpreted as the proportion of variability in the dependent variable that is explained by the model. Instead, the pseudo $\mathrm{R}^{2}$ values describe how improved a fitted model is from a null model (UCLA Statistical Consulting Group, n.d.).

For each pile impulse and all experimental trials, absolute (i.e., not normalized) received $\mathrm{SAL}_{z-p k}$ was plotted for each alarm response type to summarize which acceleration amplitudes elicited each behavioral response type. A Kruskal-Wallis test was performed to compare differences in these received levels across response types, followed by Wilcoxon rank sum tests for pairwise comparisons at a significance level of $\alpha=0.005$ (after a Bonferroni correction for 10 pairwise comparisons). Response types versus received level were also ranked for regression analysis with a Spearman's Rho test, at significance level $\alpha=0.05$, classifying "Inking" as the "strongest" response (Rank 1), followed by "Jetting", "Startle", "Body pattern change", then "No response" as the "weakest" (Rank 5).

\section{RESULTS}

Tank acoustics and sound exposures

Background sound levels of the tank were low, starting at a PSD of ca. $80 \mathrm{~dB}$ re 1 $\mathrm{uPa}^{2} / \mathrm{Hz}$ at $20-100 \mathrm{~Hz}$ and decreasing in a logarithmic fashion down to ca. $50 \mathrm{~dB}$ re $1 \mathrm{uPa}^{2} / \mathrm{Hz}^{2}$ at 600-1000 Hz (Fig. 3A). Acceleration levels of ambient sound in the tank and silent playback were below the self-noise floor of the accelerometer, evidenced by flat power spectra of these recordings at $-70 \mathrm{~dB}$ re $1\left(\mathrm{~m} \mathrm{~s}^{-2}\right)^{2} / \mathrm{Hz}$ (not shown). This relatively quiet tank environment enabled us to isolate and assess potential effects of water-borne pile driving noise on squid while minimizing extraneous stimuli that are found in natural field environments. The PSD curves of pile driving pulses in the tank indicated that noise playback created a substantially elevated sound field (by up to $50 \mathrm{~dB}$ ) and this noise was generally similar among the three playback files, with some differences in spectral shape between pressure and acceleration 
metrics (Fig 3A-B). The highest received pile driving pulse energy was between $100-300 \mathrm{~Hz}$, a range within which $D$. pealeii has relatively high sound sensitivity (Mooney et al., 2010; Mooney, Samson, et al., 2016). Across the frequency range of 20-1000 Hz, acceleration PSD of pile driving pulses was 20-30 dB higher than that of inter-pulse intervals. Spectral curves of sound pressure of pile driving pulses received in the tank had roughly similar shapes but lower amplitudes (by 20-40 dB) compared to those received in the field $0.5 \mathrm{~km}$ from the pile installation. These sound pressure levels in the tank were in the range of levels typically present in the water column 2-4 km away from the Block Island Wind Farm piles (Amaral et al., 2018). Acceleration PSD of pile driving pulses in the tank was about $20 \mathrm{~dB}$ higher than acceleration in the field at most frequencies from $20-1000 \mathrm{~Hz}$, and about $40 \mathrm{~dB}$ higher near $300 \mathrm{~Hz}$. Median peak particle velocity (Euclidian norm) across all tank recording positions and files was -40 (IQR: -43 to -37$) \mathrm{dB}$ re $1 \mathrm{~m} \mathrm{~s}^{-1}$, compared with measured peak particle velocity of $-70 \mathrm{~dB}$ re $1 \mathrm{~m} \mathrm{~s}^{-1}$ at about $1 \mathrm{~m}$ above the seabed and $-60 \mathrm{~dB}$ re $1 \mathrm{~m} \mathrm{~s}^{-1}$ at the seabed, $0.5 \mathrm{~km}$ from the pile (Amaral et al., 2018). A prior study that modeled peak particle velocity propagation from simulated pile driving sound predicted $-40 \mathrm{~dB}$ re $1 \mathrm{~m} \mathrm{~s}^{-1}$ at the seabed, $150 \mathrm{~m}$ away from the pile (James $\mathrm{H}$. Miller et al., 2016). This model utilized the same pile dimensions as those used for the BIWF, and same water depth (26 m) as at the BIWF site. Importantly, acoustic propagation of pressure and particle motion from pile driving activity in the field varies based on many variables, including but not limited to pile dimensions and rake (angle with respect to the seabed), hammering equipment and hammer energy, bathymetry, and seabed properties. This acoustic propagation varies widely over space and time, thus sound levels of pile driving noise at one point are not representative of levels at other locations and times.

There was considerable spatial variation in sound levels throughout the tank, with a trend of higher amplitudes near tank walls compared to the center of the tank (Fig. 3C). Wilcoxon rank sum tests revealed that $\mathrm{SAL}_{z-p k}$ for impulses of Pile1 was significantly lower than that for Pile2 $(z=-7.7, P<0.001)$ and Pile3 $(z=-7.6, P<0.001)$, but there was no significant difference in $S A L_{z-p k}$ between Pile2 and Pile3 $(z=0.074, P=0.94)$. Significant results may reflect high statistical power ( $n=224$ calibration positions) and these differences were small in magnitude; notably there was substantial overlap of PSD curves for each pile driving noise file 
(Fig. 3B) and high overlap of $\mathrm{SAL}_{z-p k}$ across calibration recording positions, with medians of 14 (IQR: 11-16), 17 (IQR: 14-20), and 17 (IQR: 14-20) dB re $1 \mathrm{~m} \mathrm{~s}^{-2}$ for Pile1, Pile2, and Pile3 respectively. Therefore, the three pile driving files were combined for subsequent behavior analyses. Estimated absolute (not normalized) $S A L_{z-p k}$ of pile driving impulses received by squid over the first minute of noise playback ranged from 4 to $24 \mathrm{~dB}$ re $1 \mathrm{~m} \mathrm{~s}^{-2}$ (mean of $15 \mathrm{~dB}$ re $1 \mathrm{~m}$ $\mathrm{s}^{-2}$ ), reflecting the spatial variability in the tank as seen in Fig. 3C. For normalized received levels, $\mathrm{SAL}_{z-\mathrm{pk}}$ over the first minute of noise playback ranged from 2 to $24 \mathrm{~dB}$ re $1 \mathrm{~m} \mathrm{~s}^{-2}$ (mean of $15 \mathrm{~dB}$ re $1 \mathrm{~m} \mathrm{~s}^{-2}$ ). Spearman's rank correlation was used to test for statistically significant trends in received $S A L_{z-p k}$ over this first minute of playback. Combining all Day 1 and Day 2 trials (27 trials total), Spearman's rank correlation with normalized received levels indicated a nonsignificant negative correlation $(P=0.08)$ of squids' received $S A L_{z-p k}$ over the first 30 pile impulses, with high variability across trials and impulse bins and a small correlation coefficient $(r=-0.06)$. For non-normalized received $S A L_{z-p k}$, Spearman's rank correlation was similarly weak, and statistically significant $(P=0.04, r=-0.07)$. Comparing the first three pile impulses only, median normalized received level was significantly lower, by about $3 \mathrm{~dB}$ re $1 \mathrm{~m} \mathrm{~s}^{-2}$, for the second impulse compared to the first impulse (Mann-Whitney $U$ test, $P<0.01$; Fig. 4 ), and levels were not significantly different between the second and third impulses (Mann-Whitney $U$ test, $P>0.017)$.

\section{Alarm responses and repeated exposures}

Nearly all squid (15 out of 16 individuals) exhibited at least one of the recorded alarm behaviors (inking, jetting, startle, body pattern change) during the first 30 pile driving noise impulses (Fig. 5A). These 30 impulses amounted to 45 to 69 s of playback, depending on interpulse interval. In contrast, only one control squid exhibited any of these behaviors during the first minute of silent playback. This control squid displayed one body pattern change, a brief flash of Bands, $7 \mathrm{~s}$ into silent playback. Proportions of squid exhibiting each of the four alarm behavior types during the first 30 impulses did not differ significantly between Day 1 and Day 2 trials, reflecting similar responses despite a ca. 24 -hr no-playback period (inking: $P=0.66$; jetting: $P=1.00$; startle: $P=1.00$; body pattern change: $P=0.45$; Fisher's exact test). Over the 
first minute of playback, a significantly greater proportion of control squid had 'No response' compared to Day 1 squid ( $P<0.001$; Fisher's exact test) and compared to Day 2 squid $(P<0.01)$. Notably, inking only occurred during experimental trials. Relatively few alarm behaviors occurred at times outside the first 30 pile impulses, and these are described in the supplementary material.

For Day 1 and Day 2 trials, responses occurred more frequently for all alarm response types during the first 30 impulses, compared to before the exposure or later during the exposure. For Day 1 trials, there were significant differences between the last minute of preexposure and the first 30 noise impulses in the proportions for all behaviors except inking (jetting, startle: $P<0.01$; body pattern change: $P<0.001$; Fisher's exact test; Fig. $5 B$ ). For Day 2 trials, there were significant differences in jetting and startle between these two time periods $(P<0.017 ;$ Fisher's exact test). Yet for Day 1 and Day 2 treatments, there were no significant differences in the proportion of trials squid exhibited any of the four alarm response types between the last minute of the pre-exposure period and pooled three subsampled periods after 5 min of noise exposure ( $P>0.05$; Fisher's exact test; Fig. $5 B)$. For Day 1 , there was a significant difference in proportion of trials with jetting between the first 30 noise impulses and pooled three subsampled periods after 5 min of noise exposure ( $P<0.01$; Fisher's exact test), and comparing these time periods for Day 2, significant differences in proportions of trials with jetting and startle responses ( $P<0.017$; Fisher's exact test). For all four alarm response types, pre-exposure behaviors were not significantly different between Day 1 and Day $2(P<0.05)$, indicating baseline behaviors were similar across experiment days.

During the first minute of playback, inking and jetting behaviors were confined entirely to the first 18 impulses, i.e., up to about $40 \mathrm{~s}$ of playback, and the majority of recorded behaviors occurred during the first eight impulses (Fig. 6). The greatest proportion of each alarm response type occurred at the first impulse and responses quickly diminished with successive impulses. There were no significant differences in proportions of any alarm behavior between Day 1 and Day 2 trials in any of the first 30 impulse time bins $(P>0.05$; Fisher's exact test). Pile driving impulse number was a significant predictor of the number of each alarm behavior over the first 30 impulses for Day 1 (inking: $P<0.05$; jetting: $P<0.001$; startle: $P<$ 
0.001; body pattern change: $P<0.01$; Poisson GLMs) and for Day 2 (inking: $P<0.05$; jetting: $P<$ 0.001; startle: $P<0.01$; body pattern change: $P<0.001$; Poisson GLMs). With the exception of body pattern change for Day 1 , the pseudo $R^{2}$ values from this analysis were relatively close to 1 (see Fig. 7 caption), suggesting the regression models provided a good fit for the trend of decreasing occurrence of each behavior type over time (Fig 7). The models predicted that first inking behavior, followed by startle responses and jetting, extinguished before the $30^{\text {th }}$ pile driving noise impulse, and predicted extinction or near-extinction of alarm-type body pattern changes by this point. Visually, high overlap of $95 \%$ confidence intervals for these GLM curves indicated that the initial response rates and decay in responses over time for inking, jetting, and startle responses were similar between Day 1 and Day 2 (Fig 7C-F). However, for body pattern changes the models predicted more responses on Day 2 for the first ten impulses than on Day 1. Additional Poisson GLMs (not shown in Fig. 7) including both pulse impulse number and treatment day as explanatory variables were run to examine the influence of day; these models also found that pile impulse number had a significant effect for all behaviors (inking: $P<0.01$; jetting, startle, and body pattern change: $P<0.001)$, but day did not have a significant effect on any of the behaviors $(P>0.05)$. For a further breakdown of alarm responses plotted per impulse number 5-15 min into exposure, and during the last minute of the pre-pile period, refer to the supplementary material (Fig. S1).

Body pattern changes were separated by chromatic components (Fig. 8, Fig. S2). A state of major alarm is indicated by All dark and Clear components, which combined made up almost half of the body pattern changes during the first minute of noise playback (45.7\%; All dark: 23.7\%; Clear 22.0\%). Chromatic components that indicate a relatively minor alarm state, White arms, Dark arms, and Dark arms/head, combined also made up nearly half of these body pattern changes during the first minute (47.5\%; Dark arms: 44.1\%; Dark arms/head: 1.7\% White arms: $1.7 \%$ ). There was only one occurrence of Dark arms/head (on impulse number 1) and one occurrence of White arms (on impulse number 27) during this minute. Dark arm stripes, also indicative of minor alarm, occurred only within the 15th minute of noise exposure. Bands, which typically indicates an attempt at crypsis (camouflage) but could also indicate a minor state of alarm, was less frequent during the first minute (8.5\%). 


\section{Alarm responses versus acceleration levels}

Analyzing over the first minute of pile driving noise playback, for Day 1 and Day 2 trials, median received $S A L_{z-p k}$ at which squid inked was greater than for any other alarm response behavior (Fig. 9A). Distributions of $\mathrm{SAL}_{\mathrm{z}-\mathrm{pk}}$ for jetting and startle responses, followed by body pattern changes, spread to lower values than those for inking. For Day 2 trials, median $\mathrm{SAL}_{z-\mathrm{pk}}$ was lowest for impulses at which "No response" occurred. A Kruskal-Wallis test indicated significant differences in $\mathrm{SAL}_{z-p k}$ among these five response categories for Day $2(P<0.05)$ but not for Day $1(P>0.05)$. Pairwise tests indicated no significant differences in received $\mathrm{SAL}_{z-\mathrm{pk}}$ among the four alarm response types and 'No response' for Day 1 or Day 2 trials (Wilcoxon rank sum tests, $P>0.005)$. Regression analyses of these same data were performed after ranking the ordinal alarm response categories with "Inking" being the 'strongest' response, followed by "Jetting", "Startle", "Body pattern change", and "No response" being the 'weakest' response (Fig. 9B). This revealed a significant, monotonic relationship between received acceleration level and behavioral response for Day 2 , with the received amplitude decreasing as the 'strength' of the response decreased (Spearman's Rho: $r=-0.16, p<0.01$ ). For Day 1 , this regression analysis resulted in a similar trend with weaker correlation and a non-significant result (Spearman's Rho: $r=-0.03, p>0.05)$.

\section{DISCUSSION}

All squid exposed to pile driving noise responded with alarm behaviors, indicating squid detected and were dramatically influenced by this noise. The responses (inking, jetting and pattern change) were clearly identifiable. Such behaviors are used for escaping predators and communication with conspecifics, including in reproductive contexts (Hanlon et al., 1999; Shashar \& Hanlon, 2013). This study sought to focus on individual squid to assess the overall likelihood of responses without the complexities of ecological interactions (yet). Hence we did not test for disruption to inter- or intraspecific communication in the present study, yet we found noise affected fundamental behaviors (e.g., body pattern changes, evasion) typically enacted by squid as part of communication and ecological interactions. Therefore, noise 
exposure could potentially influence these fundamental communication pathways. Given the ecological and commercial importance of squid, the potential of such an impact should be of concern to fishers, and those who seek to manage squid stocks and the ecosystems with which they interact.

The files of pile driving used for playback in the present study were from hydrophones about $1 \mathrm{~m}$ from the bottom at $500 \mathrm{~m}$ away from pile driving sites. Particle acceleration values in our experiment tank reflected those squid may experience at and near the seabed within $500 \mathrm{~m}$ from offshore pile driving sites, based on the fact that particle acceleration values in our experiment tank exceeded those measured in the field both at and just above the benthos 500 $m$ away. However, there is a paucity of measured data on water-borne (and sediment-borne) particle motion from offshore pile driving; indeed, there are few published field-based particle motion data sets for any sound source (Roberts et al., 2016). A better understanding of particle motion propagation in the field is needed before accurate predictions can be made on how squid are differentially impacted by pile driving noise with distance from the pile and proximity to the seabed. Notably, given the inherent complexity of acoustic propagation in small tanks (Rogers et al., 2016), it was not possible to recreate the exact pile driving noise field present in the squids' natural environment. The noise field in our tank was our best approximation of water-borne noise from pile driving, given available resources. Future tests should examine squid behavior in the field, where propagation of pile driving noise playback may better reflect signals from real pile installations.

Due to the high spatial variation of sound acceleration in the tank (Fig. $3 \mathrm{C}$ ), the squid probably could not seek refuge in locations of the tank subject to lower particle acceleration levels. The highly variable trends in normalized $\mathrm{SAL}_{z-\mathrm{pk}}$ received by the squid (estimated based on squids' location at the onset of each noise pulse) suggest squid did not, or could not, remain in locations of the tank with lower particle acceleration magnitude. Possible locomotion of squid at the playback onset towards tank locations with lower acceleration levels is suggested by the $3 \mathrm{~dB}$ decrease in median normalized received levels from the first to the second pile impulse. However, this difference may be attributed to the fact that the normalized levels recorded in the tank were on average (across all files and calibration recording positions shown 
in Fig. 3C) $2 \mathrm{~dB}$ higher for the first impulse compared to the second impulse. This was an amplification of the first impulse near the beginning of the file that was not present in the recording used for playback, perhaps caused by distortion of playback through the amplifier and speaker. Although squid positions were not tracked beyond the first minute of playback, it seems unlikely that clear behavioral avoidance of areas of higher sound levels would be determined in this experimental setup because of the complex particle acceleration field in the tank, with a non-monotonic amplitude gradient with distance from the speaker, and because of the small tank size relative to the animal. Future studies in larger enclosures, and ideally on unconfined wild squid, would be better suited for assessing directional behavioral responses to pile driving noise.

The alarm and escape behaviors observed during noise playback are characteristic squid responses to perceived predatory threats (Hanlon \& Messenger, 2018; Staudinger et al., 2011). Squid rely on these 'secondary defenses' to survive predatory attacks when primary defenses, e.g., camouflage, fail to prevent detection by the predator (Staudinger et al., 2011). Inking serves to confuse and deter predators during an escape sequence (Derby et al., 2013; Staudinger et al., 2011; Wood et al., 2010), and may act as an alarm cue for conspecifics (Wood et al., 2008). Various body pattern changes and startle movements are employed to bluff or intimidate predators, whereas jetting is the primary means of escape. Startle responses such as forward lunging and chromatic visual displays also occur during aggressive interactions with conspecifics (Hanlon et al., 1999), but given the solitary experimental setup, it is likely that squid in the present study were reacting to perceived threats (i.e., particle acceleration impulses) unrelated to conspecifics. Chromatic components of body pattern changes indicated relative states of alarm, with Dark arms (indicating a minor state of alarm) the most common during the first minute of noise exposure, and All dark and Clear components (indicating a relatively stronger state of alarm) being the second and third most common, respectively. These components, along with flashes of Dark arms/head, White arms, and Dark arm stripes, may all be employed to startle and bluff squids' would-be predators. Bands displayed in sync with noise impulses could either represent brief attempts at crypsis or may be incorporated with other chromatic changes as part of an anti-predator 'bluff' sequence. 
Squid exhibited similar proportions of alarm responses between Day 1 and Day 2 trials over the first $30 \mathrm{impulses,} \mathrm{and} \mathrm{in} \mathrm{both} \mathrm{groups} \mathrm{alarm} \mathrm{responses} \mathrm{diminished} \mathrm{quickly} \mathrm{over} \mathrm{time,}$ with GLMs indicating all recorded behavior types but body pattern changes were extinguished by the $30^{\text {th }}$ impulse. Regression analysis predicted near (but not total) extinction of alarm-type body pattern changes by the $30^{\text {th }}$ impulse, but this model did not consider body pattern changes occurring at later subsamples of the noise exposure period. This suggests that responses, although rare, are likely to still occur across the entire pile driving exposure. Given that body pattern changes persisted the longest, visual communication systems would potentially be disrupted for much of the pile driving event. Further, there may be disruption to their predator-avoidance system, even if escape responses are not clearly exhibited.

Reduction in alarm responses over several noise impulses indicates increased tolerance over time to the noise source, and suggests these squid may have behaviorally habituated. This pattern of decreased responses, and re-sensitization after a rest period, would be expected in response to natural stimuli as well, such as stimuli from potential predators wherein there is no reinforcement of repeated stimulation or escalation of threat. True behavioral habituation is defined as a learned, persistent reduction of an individual's response to a stimulus repeated over time, as individuals learn the stimulus has neither adverse nor beneficial consequences (Bejder et al., 2009). Because we did not test for learning mechanisms in the present study, we cannot with certainty define the decrease in responses over time as "habituation". Day 1 and Day 2 response rates occurred at statistically similar proportions, indicating squid had resensitized to the noise source by $24 \mathrm{~h}$ after the first exposure. Poisson GLMs indicated that decreases in responses occurred at similar rates during the noise exposure on Day 2 as on Day 1. This suggests a lack of long-term increased tolerance (in terms of alarm responses) after extended gaps in pile driving bouts, i.e., the reduction in responses by squid on Day 1 did not impact response rates on Day 2. We do not know the effect of this increased tolerance to subsequently detecting and responding to biologically relevant stimuli. Perhaps this saturation of response rates during pile driving may impair or impede signaling and responses to predators or conspecifics.

The increased tolerance observed in the present study reflects that seen in previous 
studies that reported rapid habituation of squid to sound and visual stimuli. Authors of a recent study played $200 \mathrm{~Hz}$ tones to D. pealeii in a similar experimental setup to the present study (Mooney, Samson, et al., 2016). These tones were repeated once every minute and resulted in logarithmic decreases of jetting and body pattern changes over time. An earlier study investigated habituation of a different squid species, Lolliguncula brevis (Blainville 1823), to presentations of a visual predator model once per minute (Long et al., 1989). The duration of body pattern changes and number of jets diminished quickly, with jetting extinguished by the $10^{\text {th }}$ trial. The authors found complete recovery of both behaviors after a $1 \mathrm{~h}$ rest period. Further, sets of stimulus presentations spaced with $1 \mathrm{~h}$ rest periods contributed to longer-term habituation that carried over to the second day of trials. This result suggests squid alarm responses to pile driving noise may recover after rest periods much shorter than $24 \mathrm{~h}$, and shorter rest periods may lead to faster increases in tolerance at the onset of each noise exposure period.

In a typical offshore construction scenario such as that for the BIWF, several piles may be driven per day, each lasting on average 30-90 min, with rest periods in between ranging from several minutes to several hours (Amaral et al., 2018). It may be informative for future experiments to test for recovery and habituation of alarm responses after shorter rest periods. Additionally, ramp up procedures might reduce the occurrence rate of startle responses. In a ramp up procedure, hammer blow energy, which is proportional to emitted noise intensity from the pile, is gradually increased up to full power. Although effectiveness of pile driving ramp up in reducing adverse impacts on marine animals has not been systematically tested, it is sometimes employed as a mitigation technique for pile driving (Bailey et al., 2014); thus future research should consider its influence on behavioral responses.

As noted above, we did not test for learning mechanisms, thus reduced response rates may have occurred due to mechanisms other than habituation. One such mechanism could be that over time squid failed to exhibit inking or locomotor responses due to depleted ink or energy reserves. This is unlikely given the small number of inking and jetting behaviors observed during trials, and because many squid jetted and inked during handling after the trials ended. Another potential mechanism is the occurrence of an auditory threshold shift (TS), i.e., 
temporary or permanent hearing loss after repeated noise exposure. For context, the sound pressure levels over the first minute of the present study were substantially lower than thresholds suggested for TS in fish caused by pile driving noise (Popper et al., 2014). However, because no comparative data exist (to the authors' knowledge) on TS in cephalopods or other invertebrates or on TS in terms of particle motion, and because physiological variables were not measured in the present study, the possibility that physiological mechanisms contributed to reduced behavioral responses over time cannot be ruled out.

In addition to its dependence on the number of noise impulses over time, there was a dependence of alarm response occurrence on received acceleration levels, with squid exhibiting "No response" having received significantly lower $\mathrm{SAL}_{z-p k}$ during analyzed impulses than on impulses to which they exhibited an alarm response. Lower received $\mathrm{SAL}_{z-p k}$ correlated with ranked alarm responses of lower 'strength' (i.e. minor alarm responses such as startle and body pattern changes or no response, compared to 'stronger' inking and jetting responses). Out of the four alarm response types, inking occurred at a higher median $\mathrm{SAL}_{z-p k}$ than jetting or

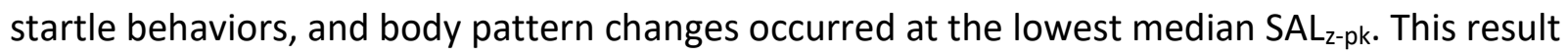
matches the trend of amplitude-dependent alarm responses observed previously for this species in response to sinusoidal tones, where inking occurred at the highest sound levels (and lowest frequencies), body pattern changes occurred at sound acceleration levels an order of magnitude below inking, and jetting and startle behaviors occurred at sound acceleration levels between these at low frequencies (Mooney, Samson, et al., 2016). This suggests that, as for artificial tones, higher-amplitude (or potentially closer) pile driving noise is more likely to elicit locomotor escape responses including inking and jetting, which are naturally employed to avoid capture by predators, versus lower-amplitude impulses that are more likely to elicit body pattern changes or no response.

\section{Comparisons with related noise studies}

To our knowledge, this is the first study to test effects of aspects of pile driving noise on any cephalopod, and follows a comparative study that examined impacts of a separate type of anthropogenic noise, air gun pulses, on squid (Fewtrell \& McCauley, 2012). Their work found 
that seismic air gun arrays, which passed over caged groups of Sepioteuthis australis, elicited alarm responses similar to those in the present study, including inking, jetting, and body pattern changes. In a study with cuttlefish (Sepia officinalis), in-lab playbacks of boat noise elicited body pattern changes, specifically increased frequency of the Raised arms posture and changes in chromatic components, as well as time spent swimming (Kunc et al., 2014). These changes suggested an increased state of alarm and stress during noise playback. Startle responses to substrate-borne vibration have also been observed in the mussel Mytilus edulis and hermit crab Pagurus bernhardus (Roberts et al., 2015, 2016). Though the present study only considered water-borne particle motion, pile driving also produces large substrate-borne vibration (Roberts \& Elliott, 2017). Doryteuthis pealeii naturally rests on the substrate and would potentially sense this "ground-roll" as well; accordingly, future studies on this species should examine the influence of substrate-borne vibration.

Squid schooling and shoaling behaviors could be disrupted during impulsive noise, as has been demonstrated in fish. For example, schools of European sea bass (Dicentrarchus labrax) responded to anthropogenic noise impulses by increasing swimming depth and school cohesion, both considered primary stress responses (Neo et al., 2014, 2015). Decreased school cohesion has also been observed in D. labrax during pile driving noise, which might increase predation risk (Herbert-Read et al., 2017a). Future studies with groups of squid should monitor changes in schooling behaviors, in addition to fighting, visual displays, and other behaviors naturally occurring in agonistic and reproductive scenarios. Since pile driving activity could occur during the day or night and wild $D$. pealeii occupy different parts of the water column between day and night (more benthic and schooling during the day, more pelagic and dispersed at night), differences in behavior during these periods should also be considered. Diel differences in responses to simulated noise impulses have been found in fish that naturally exhibit diel differences in schooling behavior (Hawkins et al., 2014).

\section{Ecological implications and conclusions}

Short-term effects such as startle responses and subsequent increased tolerance or habituation are often dismissed as ecologically irrelevant but there is an increasing awareness 
of the effects such responses may incur (Bejder et al., 2009). We suggest several hypotheses for ecological impacts that can be drawn based on the present study and previous work, which are worth investigating in future studies. First, as squid become more tolerant of a noise stimulus they might fail to exhibit alarm and escape responses in order to evade predators, possibly due to: 1) learned unresponsiveness to threat stimuli, 2) distraction by the noise source (the "distracted prey" hypothesis, see Chan et al., 2010), or 3) failure to detect particle motion associated with important signals (e.g., predator sounds) in the presence of interfering noise, a phenomenon termed "masking". Squids' initial natural responses to a predator are often crypsis or schooling; short-term startle responses have the potential to disrupt schooling or cause animals to reveal themselves to predators. In both cases they are more vulnerable to predation. The aforementioned habituation study on the squid L. brevis found habituation specificity of this species to visual predator models, meaning that after habituating to one model, the squid showed elevated jetting and body pattern change responses when presented with a new, different model (Long et al., 1989). Squid that have diminished responses to pile driving noise may restore behaviors in response to new sound or visual stimuli, such as those of predators, however this mechanism should be empirically evaluated. Additionally, exposure to noxious stimuli may increase squids' alertness to predators. After physical injury, D. pealeii exhibited increased responsiveness to black sea bass (Centropristis striata) attacks, initiating alert and flight reactions sooner and at longer distances from the predator (Crook et al., 2014). It is yet unclear whether squid would similarly be sensitized (or desensitized) to predatory threats during or after noise exposure.

Although it is not known how squid utilize natural sound cues, squid possess sets of hair cells along the external surface of their skin which detect nearby water movements and play an important role in successful predator evasion (York \& Bartol, 2014). Squid also possess internal statocyst structures known to detect particle motion, specifically acceleration (Budelmann, 1990; Mooney et al., 2010). Exposure to pile driving noise could potentially interfere with the detection of particle motion cues from nearby, swimming predators via masking though both these sensory systems, though masking effects have not yet been investigated in any cephalopod. 
Although the present study did not test for noise-amplitude dependent alarm responses, inking and jetting are expected to occur more frequently in squid as the amplitude of low-frequency noise increases (Mooney, Samson, et al., 2016). In future studies, when significant impacts of pile driving noise on ecologically relevant behavioral and physiological variables are found, attempts should be made to establish amplitude and duration (for a given inter-pulse-interval) thresholds for adverse effects to likely occur. Such thresholds would better inform regulations for acceptable limits on pile driving activity in areas utilized by ecologically and economically important species such as $D$. pealeii.

Our study is the first to demonstrate behavioral effects of pile driving noise on any cephalopod species, and focused on a commercially important squid species whose range overlaps with areas where marine construction projects involving pile driving may occur in the near future. We observed a variety of alarm responses associated with anti-predator reactions at the onset of pile driving noise. Squid had rapidly diminished alarm responses within the first minute of noise exposure in all trials, and had re-sensitized to the noise after a 24-h rest period. Diminished predator-defense and escape behaviors may alter squid susceptibility to predation, depending on potential factors such as squids' habituation specificity and their predators' responses to noise. Although caution must be taken when extrapolating lab-based results to free-swimming wild animals that may potentially escape a noise source, controlled lab-based studies are useful for analyzing such interactions without the influence of confounding environmental variables. That said, to conclusively test ecological implications of noise exposure, field studies are also needed, in which acoustic conditions are more realistic and squid are less confined, and thus allowed to behave more naturally. Many other ecologically important behaviors such as mating and foraging behavior remain to be examined, along with potential physiological changes, e.g. in respiration rate and energy expenditure. The present findings of the influence of pile driving noise on squid alarm responses will leverage future studies on behavioral and physiological effects of anthropogenic noise on squid and other cephalopods, as well as impacts on these animals' ecology. 
Table 1. Number of trials (one squid tested per trial) for each of the three pile driving playback files, and control trials.

\begin{tabular}{cccc}
\hline Treatment & File Played & Day 1 & Day 2 \\
\hline Pile Driving & Pile1 & 6 & 6 \\
& Pile2 & 6 & 3 \\
& Pile3 & 4 & 2 \\
& Total & 16 & 11 \\
\hline Control & Silent & 7 & N/A \\
\hline
\end{tabular}




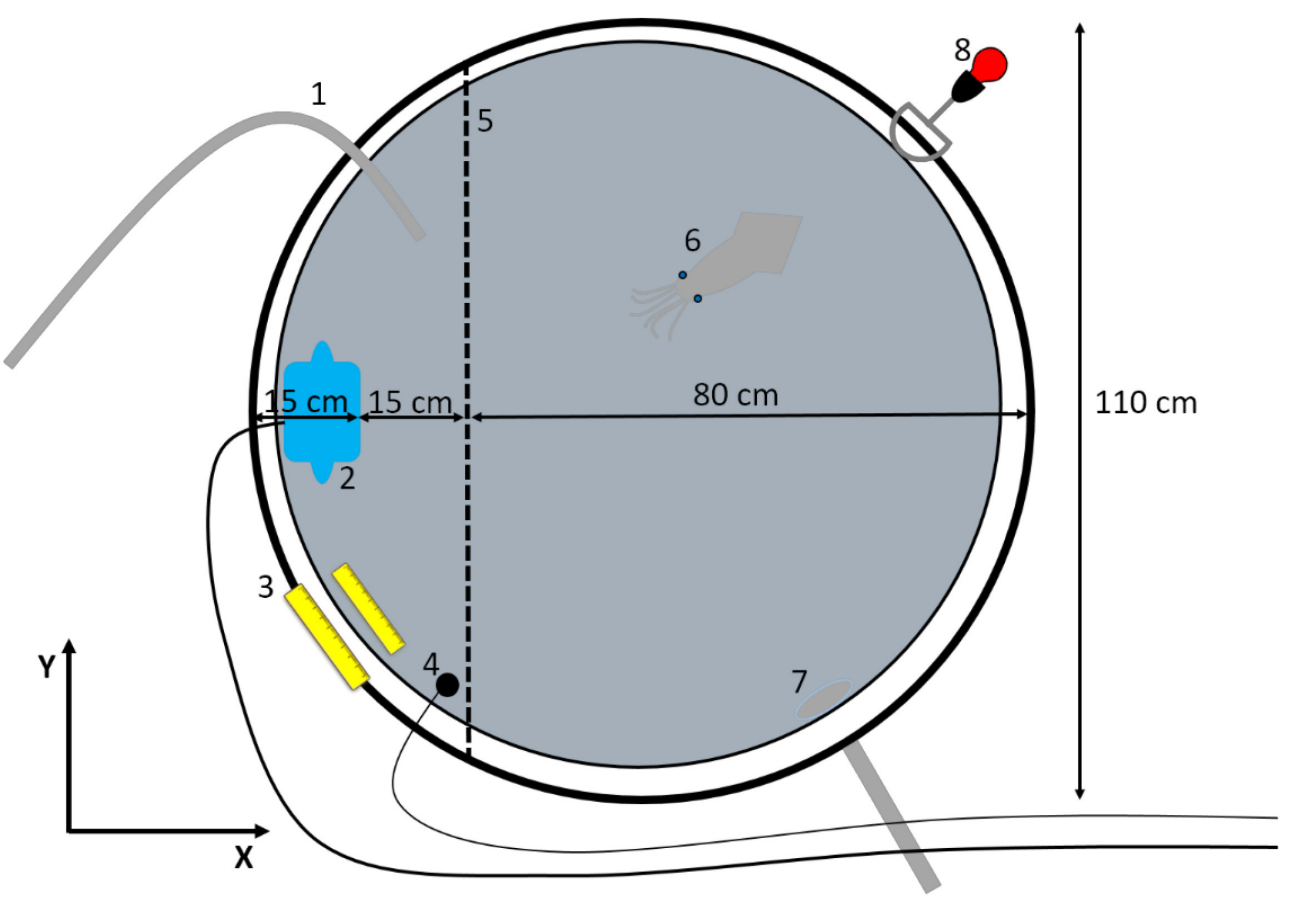

Figure 1. Top-down diagram of the experimental tank setup. 1) Water inflow hose, 2) underwater speaker, 3) calibration rulers (one on top edge of tank, one on bottom of tank), 4) hydrophone, 5) mesh barrier, 6) squid, 7) water outflow opening and pipe (in grey), 8) LED light clamped to the top edge of the tank. Measurements, but not all objects shown, are to scale. 


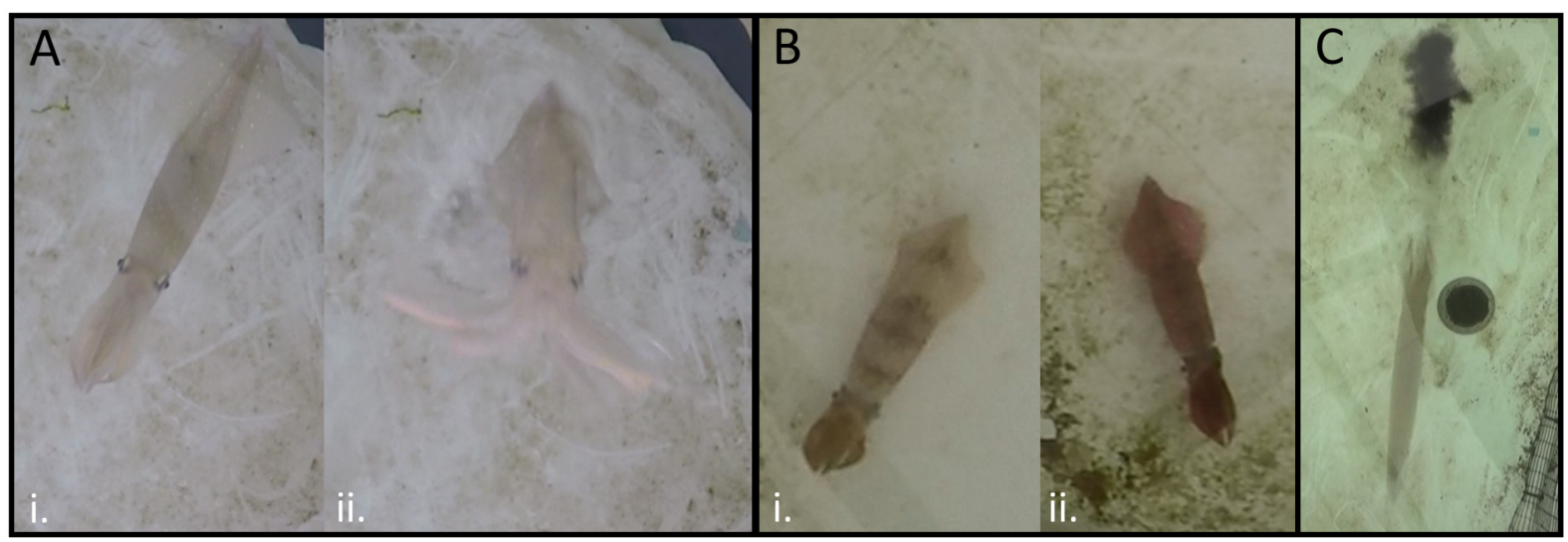

Figure 2. Examples of alarm responses observed during pile driving noise exposure. A) Calm, swimming squid with a lightly-shaded (termed Clear) body pattern before noise onset (i) that subsequently exhibited a startle response, in this case a forward lunge, during the first noise impulse (ii). B) Example of a body pattern change in another squid, in which the squid changed from Bands (i) between noise impulses to an All dark body pattern (ii) during the next impulse, indicating a change from a cryptic to an alarmed state. C) Example of simultaneous inking and jetting. See Hanlon et al. (1999) for a detailed ethogram of this species. 
Figure 3. Power spectral density (PSD) curves, in $10 \mathrm{~Hz}$ bins, for sound pressure (A) and acceleration data (B) of each pile driving file as received in the experiment tank ("Tank Pile"). Also shown are spectra for the original files used for playbacks, as received in the field $0.5 \mathrm{~km}$ away from a pile installation ("Field Pile"), and mean ambient noise in the experiment tank during the pre-exposure period ("Tank Ambient") and during the 'exposure' period of control trials ("Silent Playback"). Field recordings were provided by Jim Miller and Arthur Newhall. The PSD curves of pile driving noise in the experiment tank are from the calibration position squid were most frequently closest to ( $X=45, Y=20, Z=40$; as shown in $C$ ), and are integrated over the pulse length ('Pulse') as defined in the methods, or inter-pulse intervals (at times outside the pulse length). The PSD curves for the field data were also calculated over the first minute of each file. PSD levels for "Tank Ambient" and "Silent Playback" curves were calculated over the 15 min pre-exposure and 15 min control exposure periods, respectively. C) Maps of mean received zero-to-peak (z-pk) acceleration (top row) and z-pk sound pressure (bottom row) in the tank from the acoustic calibration, at each water depth (columns). Median z-pk level across pile impulses for the first minute of each of the three pile files was found, then for each recording position the mean value across files was calculated, shown here. The origin for the XY plane is the speaker. 
A

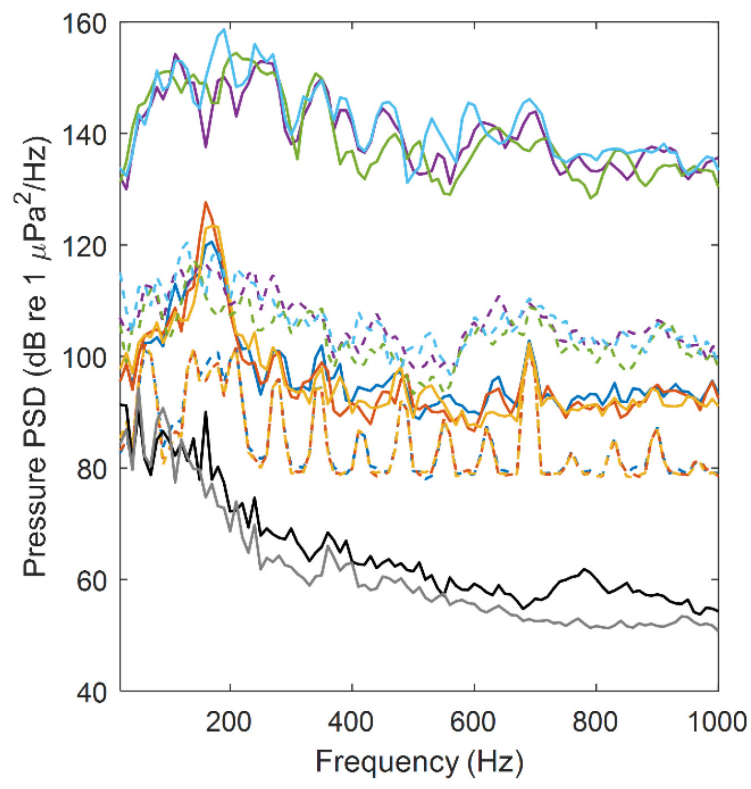

C
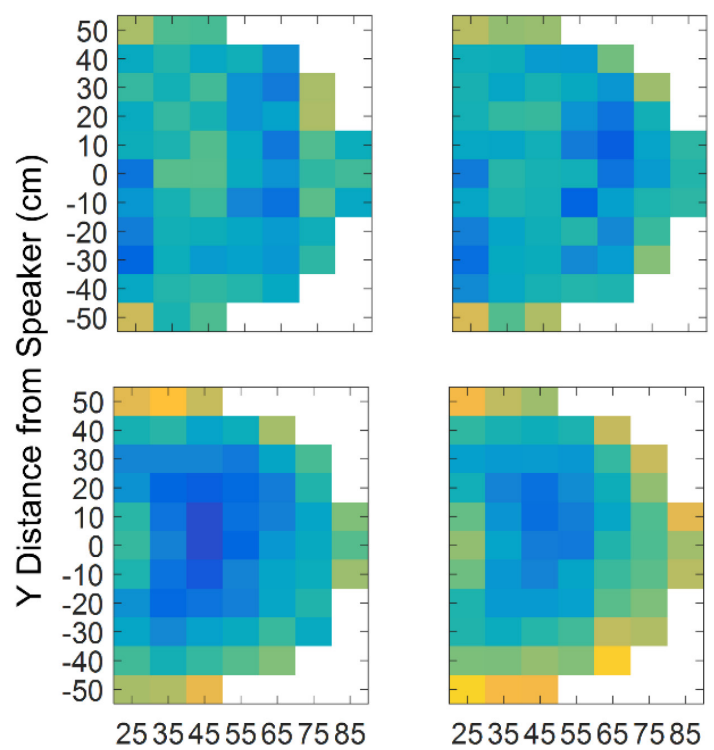

25354555657585
B

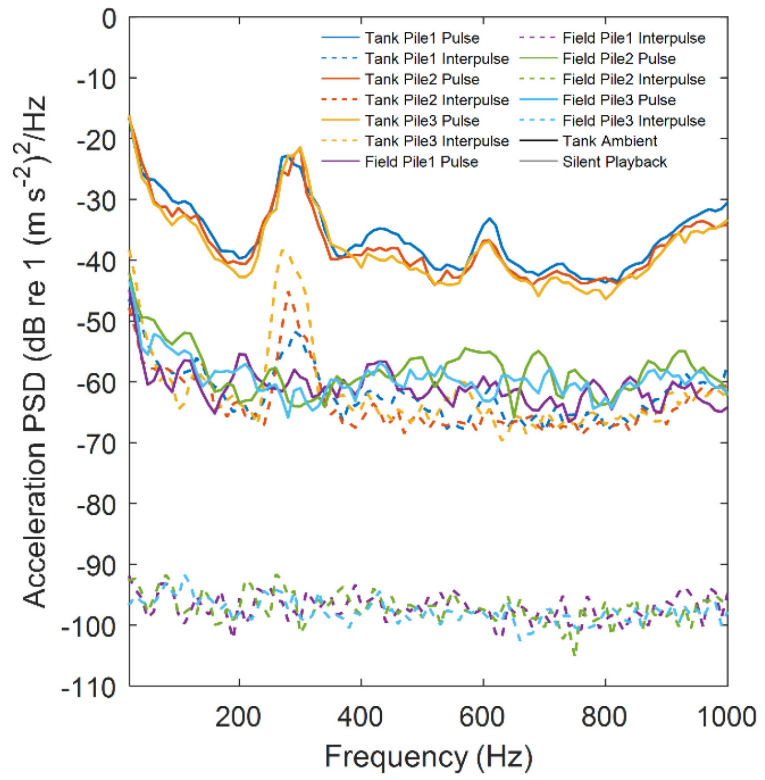

$\mathrm{Z}=30 \mathrm{~cm}$

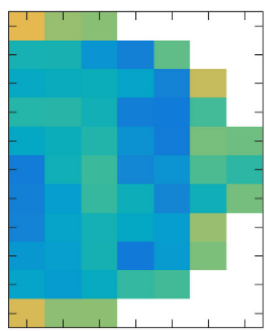

$Z=40 \mathrm{~cm}$

$X$ Distance from Speaker $(\mathrm{cm})$
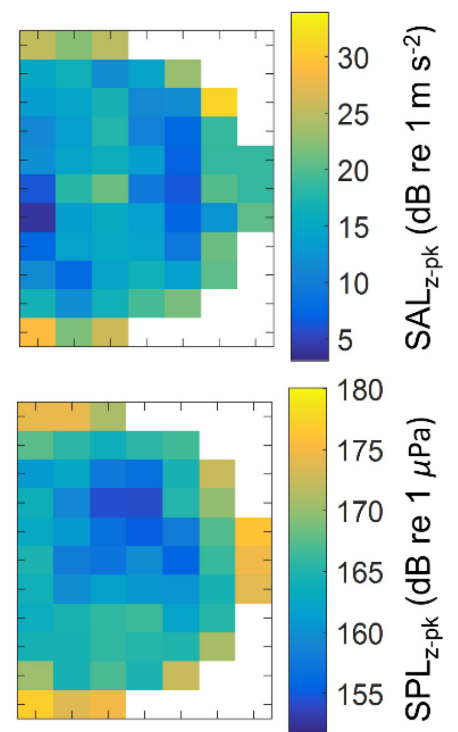

25354555657585

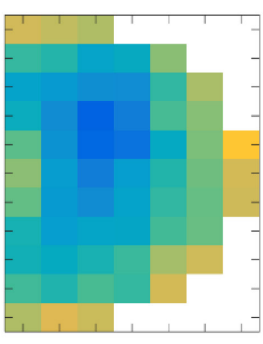

25354555657585 


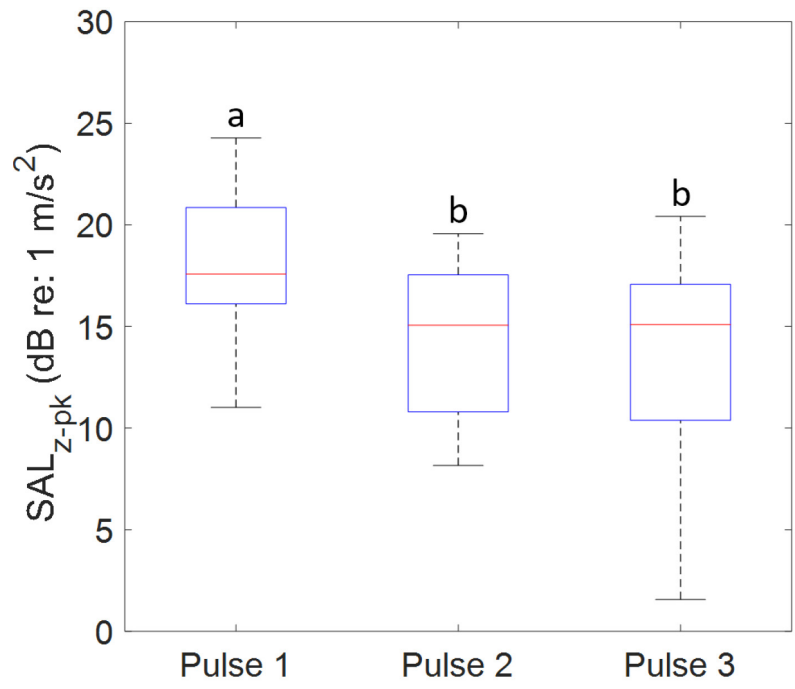

Figure 4. Normalized received zero-to-peak sound acceleration levels for the first three pulses of Day 1 and Day 2 trials combined. Red lines indicate median values, bottom and top of the boxes indicate 25th and 75th percentiles, and whiskers encompass data up to $q_{3}+1.5 \times\left(q_{3}-q_{1}\right)$ and down to $q_{1}-1.5 \times\left(q_{3^{-}}\right.$ $q_{1}$ ), where $q_{1}$ and $q_{3}$ are the 25th and 75th percentiles of the sample data, respectively. Different letters above box plots indicate significantly different groups (Mann-Whitney $U$ tests: $P<0.01$, with significance level $\alpha=0.017)$. 


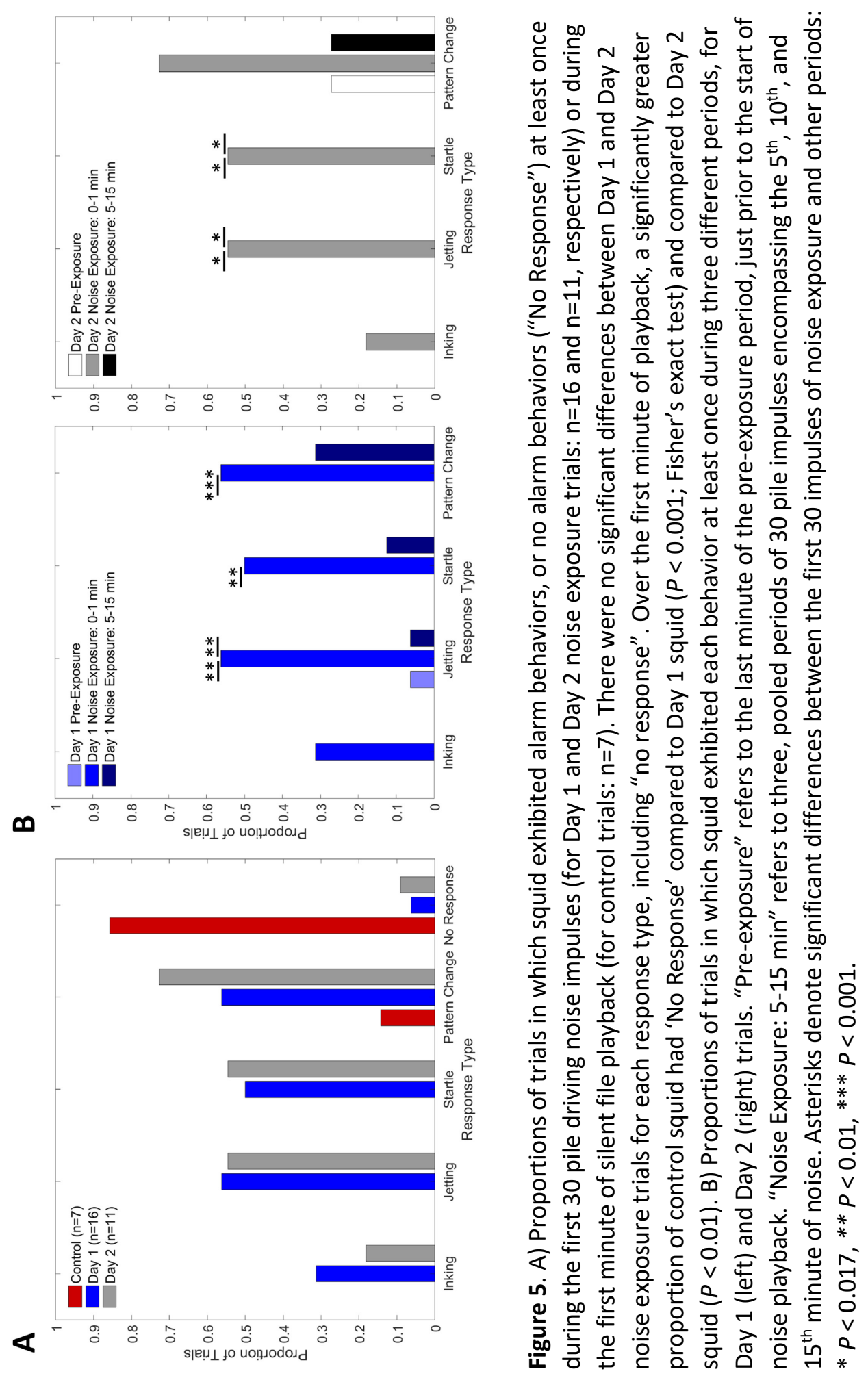




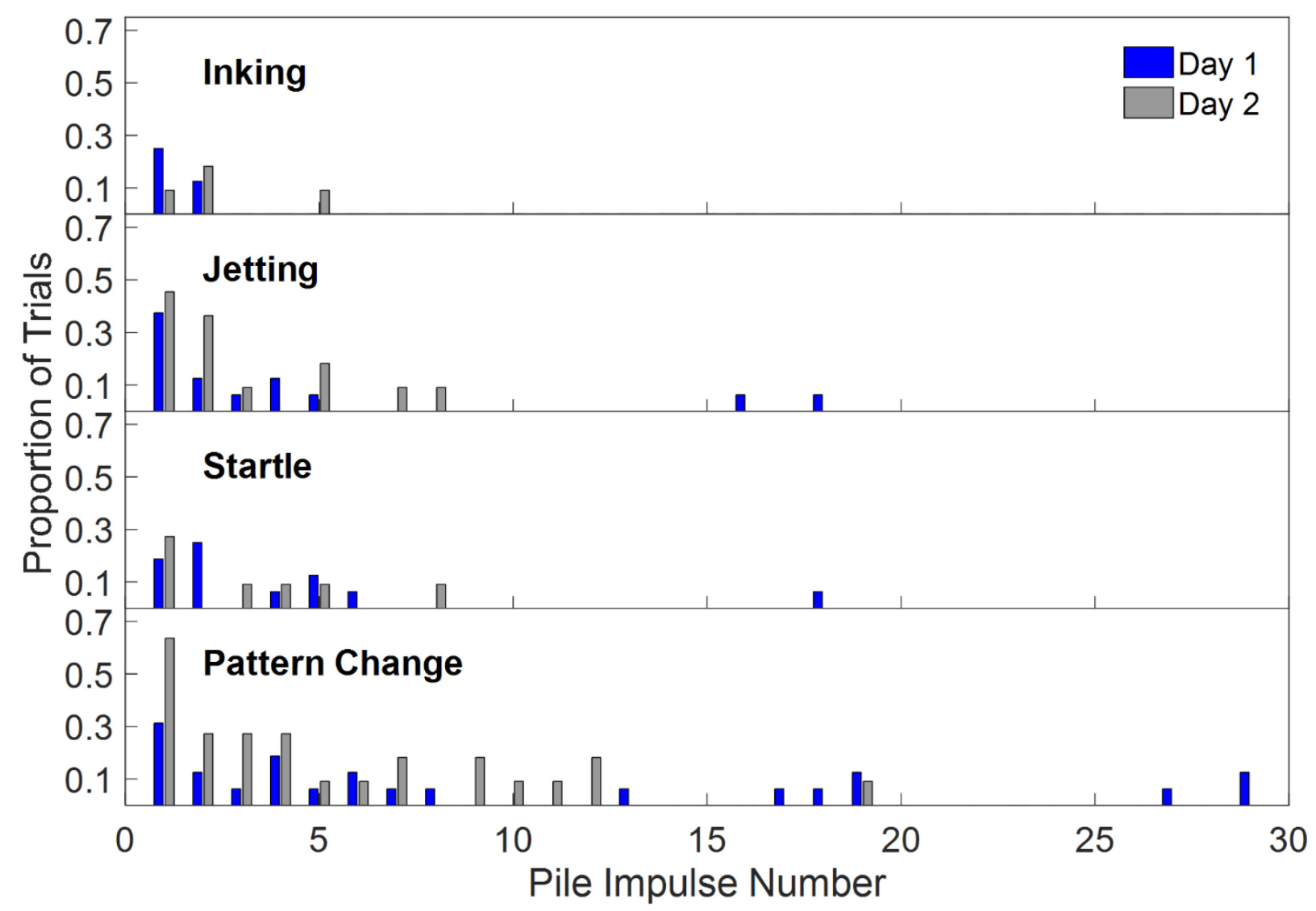

Figure 6. Proportions of trials with squid that responded with inking, jetting, startle, and body pattern change behaviors in given pile impulse time bins over the first 30 impulses, for Day 1 and Day 2 trials. 
A
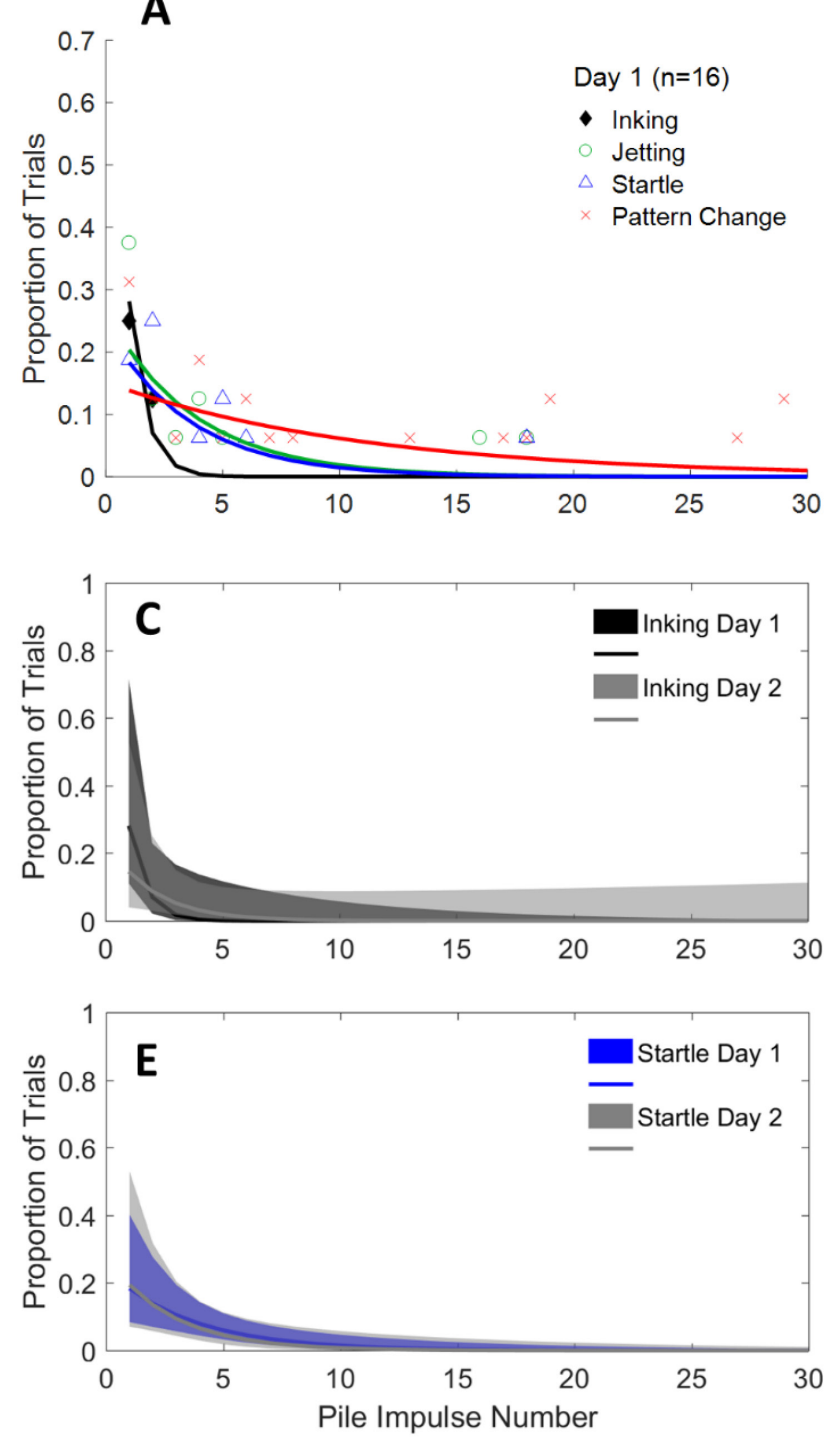

B
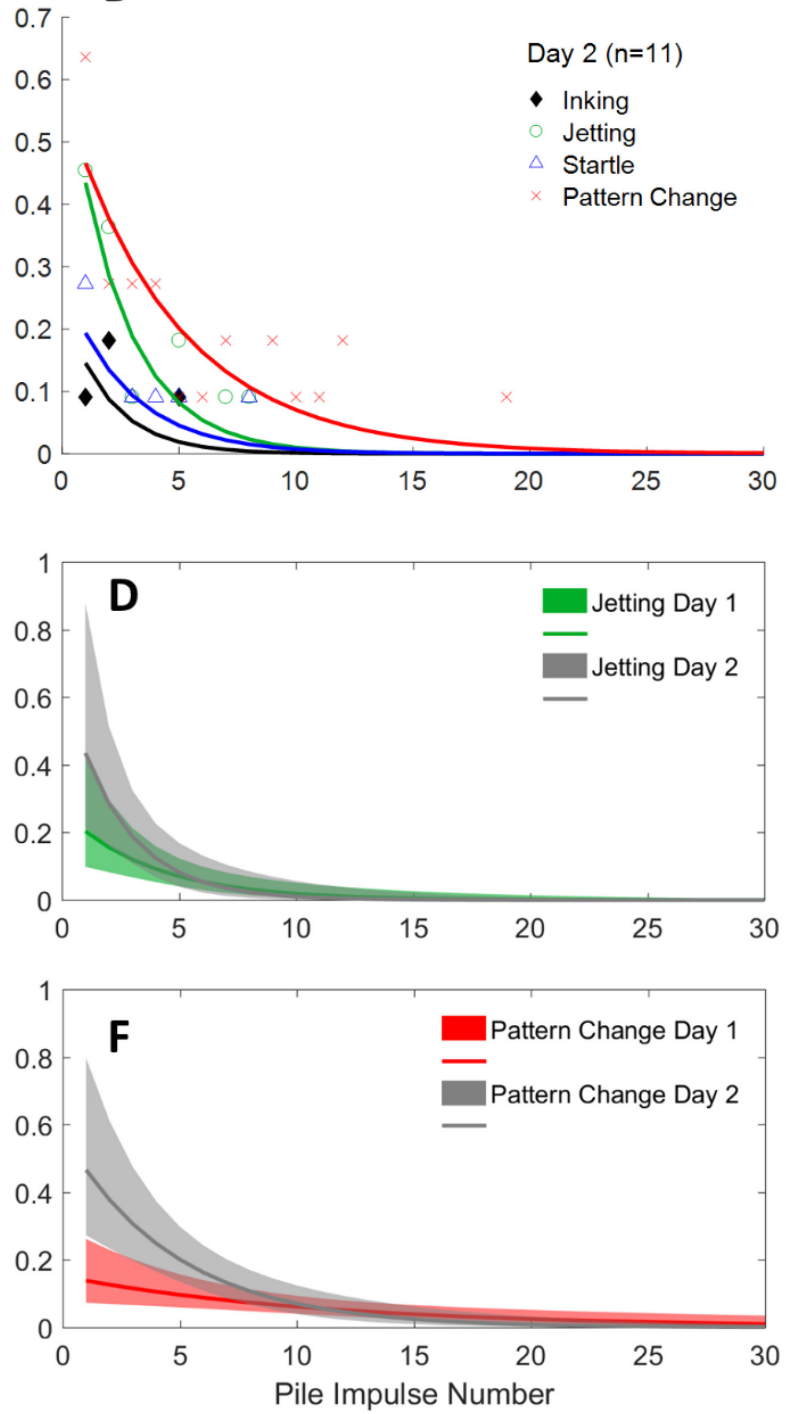

Figure 7. A-B) Proportions of alarm responses (points) across pile impulse number, with Poisson Generalized Linear Model curves overlaid for Day $1(A)$ and Day $2(B)$ trials, with the following pseudo $R^{2}$ values: Day 1 Inking $R^{2}=0.8980$, Day 1 Jetting $R^{2}=0.7138$, Day 1 Startle $R^{2}=0.6978$, Day 1 Pattern Change $R^{2}=0.3647$, Day 2 Inking $R^{2}=0.6420$, Day 2 Jetting $R^{2}=0.8504$, Day 2 Startle $R^{2}=0.6622$, Day 2 Pattern Change $R^{2}=0.8233$. C-F) Poisson GLM curves from $A$ and $B$ for Day 1 (bold solid lines) and Day 2 (bold dashed lines) for each alarm response. Corresponding thin solid lines (Day 1 ) and thin dashed lines (Day 2 ) below and above the bold lines indicate the $95 \%$ confidence regions of each curve. 


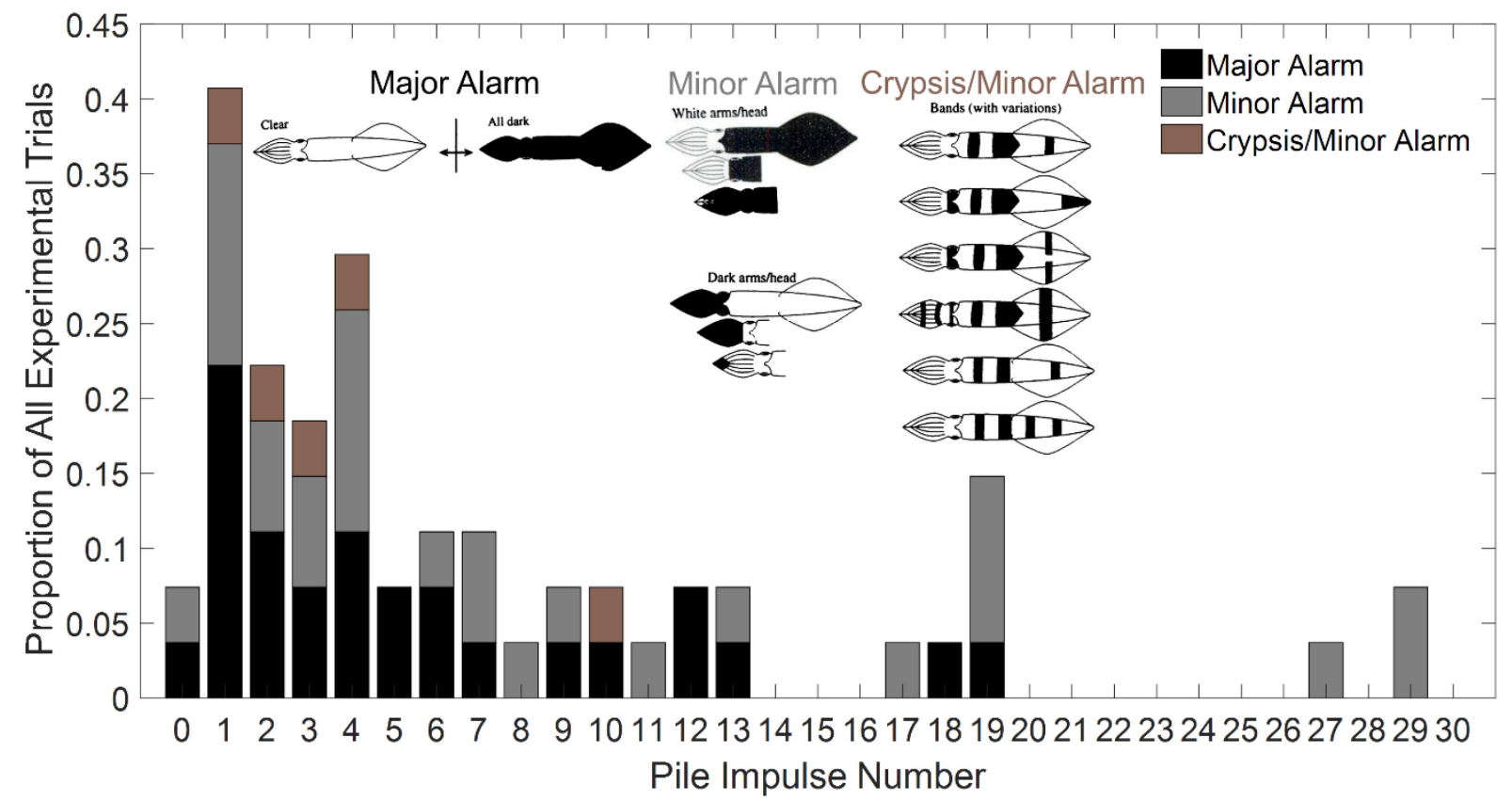

Figure 8. Proportions of all trials (Day 1 and Day 2 combined, excluding controls), for which squid exhibited various degrees of chromatic components that make up the body pattern changes reported in Fig 5-7. Drawings of each chromatic component are shown (adapted from Hanlon et al. 1999). A state of "major" alarm is indicated by Clear and All dark. "Minor" alarm is suggested by "Dark arms/head" and "White arms/head". "Bands" is classified as "crypsis/minor alarm", since it is typically used for camouflage (i.e., crypsis) but could also be presented in a minor alarm state. Proportions are displayed per pile driving impulse for the first 30 impulses. Impulse " 0 " refers to the $3 \mathrm{~s}$ fade-in period from the start of the playback file to the onset of the first pile impulse. 

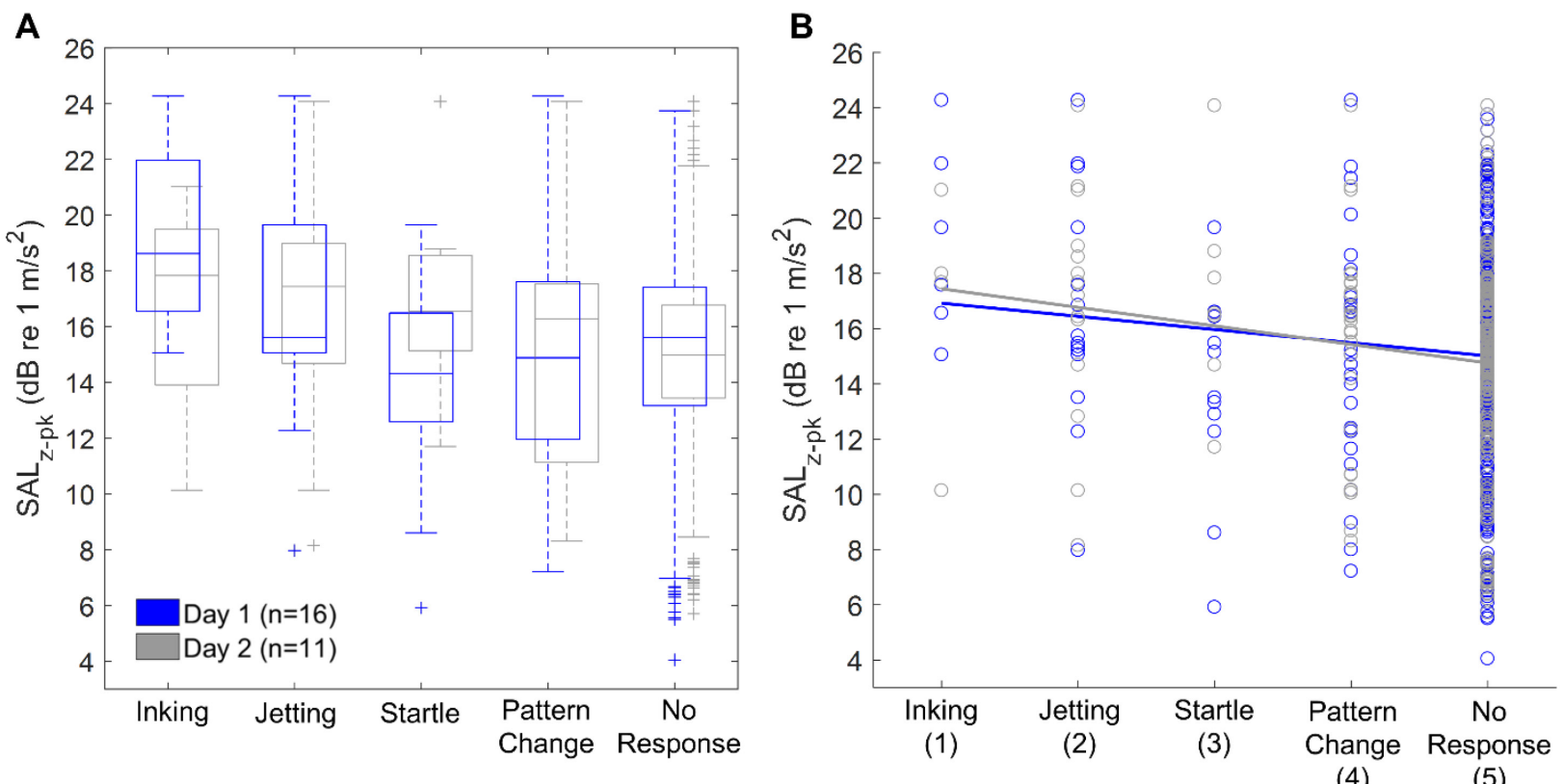

Figure 9. Received zero-to-peak sound acceleration levels (not normalized) at which squid exhibited each alarm response type or no response, as a boxplot (A) and scatterplot (B). Data are shown for Day 1 trials (blue symbols) and Day 2 trials (gray symbols) for the first minute of pile driving impulses (30 impulses for Pile1 and Pile2, or 26 impulses for Pile3). All behaviors are shown here, with more than one alarm response sometimes occurring on a given impulse during a given trial. A) Horizontal lines in the middle of the boxes indicate median values, bottom and top of the boxes indicate 25th and 75th percentiles, whiskers extend to ranges 1.5 times the $25^{\text {th }}$ and $75^{\text {th }}$ percentiles, and whiskers encompass data up to $q_{3}+1.5 \times\left(q_{3}-q_{1}\right)$ and down to $q_{1}-1.5 \times\left(q_{3}-q_{1}\right)$, where $q_{1}$ and $q_{3}$ are the 25th and 75th percentiles of the sample data, respectively. Outliers, indicated by crosses, are defined for data points outside the whisker range. B) Points indicate received acceleration levels at which each response type occurred, and linear, least-squares lines of best fit are shown for Day 1 (blue) and Day 2 (gray) trials. Including the four alarm responses and "No Response", Spearman's Rho indicated a negative and significant monotonic relationship between received acceleration level and ranked behavioral response for Day $2(r=-0.16, P<0.01)$. 
CHAPTER 3 : CHANGES IN FEEDING BEHAVIOR OF LONGFIN SQUID (DORYTEUTHIS PEALEII) DURING LABORATORY EXPOSURE TO PILE DRIVING NOISE

This chapter was originally published as: Jones, I. T., Peyla, J. F., Clark, H., Song, Z., Stanley, J. A., Mooney, T. A. (2021). Changes in feeding behavior of longfin squid (Doryteuthis pealeii) during laboratory exposure to pile driving noise. Marine Environmental Research. 165.

https://doi.org/10.1016/j.marenvres.2020.105250. The Supplementary Materials for this chapter can be found in Appendix B. 


\begin{abstract}
Anthropogenic noise can cause diverse changes in animals' behaviors, but effects on feeding behaviors are understudied, especially for key invertebrate taxa. With the offshore wind industry expanding, concern exists regarding potential impacts of pile driving noise on squid and other commercially and ecologically vital taxa. We investigated changes in feeding and alarm (defense) behaviors of squid, Doryteuthis pealeii, predating on killifish, Fundulus heteroclitus, during playbacks of pile driving noise recorded from wind farm construction within squids' habitat. Fewer squid captured killifish during noise exposure compared to controls. Squid had more failed predation attempts when noise was started during predation sequences. Alarm responses to noise were similar whether or not squid were hunting killifish, indicating similar vigilance to threat stimuli in these contexts. Additionally, novel hearing measurements on $F$. heteroclitus confirmed they could detect the noise. These results indicate noise can disrupt feeding behaviors of a key invertebrate species, and will leverage future studies on how noise may disrupt squids' vital ecological interactions.
\end{abstract}




\section{INTRODUCTION}

Underwater noise from anthropogenic activities has increased in past decades in many areas of the oceans, and accordingly, concern has grown regarding impacts of anthropogenic noise pollution on marine taxa (Andrew et al., 2011; Frisk, 2012; Gedamke et al., 2016; Haver et al., 2017; McDonald et al., 2006; Miksis-Olds et al., 2013). Increased anthropogenic activities such as shipping, seismic surveys for oil and gas exploration, and marine construction all contribute to noise pollution that can harm the physiology and behavior of marine fauna (Gedamke et al., 2016; Hawkins et al., 2015; Slabbekoorn et al., 2010). Noise-related impacts have been noted from crustaceans to cetaceans, although fewer studies have addressed invertebrates compared to vertebrates (Gedamke et al., 2016; Kastelein et al., 2016; Wale et al., 2013a). Noise can interfere with key ecological functions such as predator and prey detection, communication, and navigation (Hawkins et al., 2015; Putland et al., 2019; Stanley et al., 2017).

Impact pile driving is one such noise source of concern in marine environments. This impulsive sound arises from repeated hammer strikes that drive long piles into the seabed. These piles support foundations for coastal docks, piers, and boat slips, and offshore energy platforms such as wind farms. As the offshore wind industry expands globally (Musial et al., 2020), regulatory agencies and fishers aim to predict impacts of wind farm construction on commercially and ecologically important marine fauna. Many offshore wind projects utilize impact pile driving to install turbine supports, typically in waters less than $60 \mathrm{~m}$ deep, including the North Sea and the Northwest Atlantic continental shelf (Musial et al., 2020). Underwater sounds from offshore pile driving are high-amplitude, with peak sound pressure levels typically exceeding $200 \mathrm{~dB}$ within a range of several-hundred meters from the pile (Bailey et al., 2010; S. Lippert \& von Estorff, 2019; T. Lippert \& von Estorff, 2014). Pile driving sounds are also broadband, spanning frequencies from $<100 \mathrm{~Hz}$ to $>10000 \mathrm{~Hz}$, with peak energy usually between 100 and $2000 \mathrm{~Hz}$. These sound are far-propagating, typically detectable by hydrophones well over $10 \mathrm{~km}$ away (Amaral et al., 2018; Bailey et al., 2010). As a pile is driven, acoustic waves radiate out from the pile via multiple paths through the water column and 
substrate (Reinhall \& Dahl, 2011); therefore, benthic and interstitial animals as well as animals occupying the water column will be exposed to this noise (Roberts \& Elliott, 2017).

Pile driving sounds can have a wide variety of impacts on marine taxa. For example, they can damage organs of fishes (Casper, Smith, et al., 2013; Casper, Halvorsen, et al., 2013; Halvorsen et al., 2011), and cause temporary reductions in hearing sensitivity, as observed in harbor porpoises and the harbor seal Phoca vitulina (Kastelein et al., 2016, 2018). Behavioral changes caused by impulsive anthropogenic noise (including, but not limited to pile driving) are diverse, and include directional swimming responses of fishes and mammals away from the noise source (Aarts et al., 2018; Graham et al., 2019; Mueller-Blenkle et al., 2010), alarm responses (e.g., startle and escape behaviors) and changes in fishes' schooling behaviors, which are important defenses against predators (Hawkins et al., 2014; Herbert-Read et al., 2017a; Neo et al., 2015).

Relatively few studies have investigated changes in aquatic animals' feeding and foraging behavior during noise. Sustained reductions in animals' feeding behaviors due to anthropogenic stressors could lead to reduced survival, especially in regions with patchy prey distribution or limited prey abundance. Some studies have reported reduced foraging activity of marine mammals during pile driving, sonar, and vessel noise (Aarts et al., 2018; Erbe et al., 2019; Miller et al., 2015). In studies on fishes and invertebrates, exposure to boat noise and white noise has resulted in reduced prey capture rate, increased food handling or discrimination error, and decreased time spent foraging (Ivanova et al., 2020; Mensinger et al., 2018; Purser \& Radford, 2011; Sabet et al., 2015). While the vast majority of studies on feeding behaviors during noise have focused on marine mammals or fishes, comparatively few have focused on marine invertebrate taxa, despite a growing number of invertebrates known to detect acoustic cues in (Carroll et al., 2017; Popper \& Hawkins, 2018; Samson et al., 2016) and their high biomass and central ecological role in ocean ecosystems (Costello et al., 2010). Cephalopods (squids, cuttlefishes, and octopuses) are a key trophic link between many top predators and smaller fish and invertebrate prey (Boyle \& Rodhouse, 2005; Hanlon \& Messenger, 2018). They are also considered sound-sensitive, demonstrating neural and behavioral responses to sounds below 1000 Hz (Kaifu et al., 2008; Mooney et al., 2010; Packard 
et al., 1990). The ecological functions of their hearing abilities remain elusive; hypothesized uses of natural sounds include prey detection, predator avoidance and navigation (Samson et al., 2016; Wilson et al., 2018). Their low-frequency hearing range overlaps with dominant frequencies of many anthropogenic noise sources, including pile driving (Slabbekoorn et al., 2010). Cephalopods are therefore at risk for noise-induced changes in hearing physiology and behavior, as has been demonstrated in a few studies.

Though studies investigating effects of anthropogenic noise on invertebrates' feeding behavior are limited, several studies have indicated adverse effects of anthropogenic noise on other behaviors, as well as on physiology, of cephalopods and other invertebrate taxa. Hair cells in hearing structures of the cuttlefish Sepia officinalis, and squids Loligo vulgaris and Illex coindetii suffered damage after 2 h continuous exposures of noise (Solé et al., 2017, 2018, 2019). Southern reef squid, Sepioteuthis australis, exhibited alarm responses, i.e., inking and jetting, during impulsive air gun noise (Fewtrell \& McCauley, 2012). Pile driving noise in a prior laboratory study elicited alarm responses in longfin squid, Doryteuthis pealeii, including inking, jetting, and body pattern changes (Jones et al., 2020). Rock lobsters, Jasus edwardsii, exposed to air gun noise had an impaired behavioral righting reflex (employed to escape predation), and had damage to statocyst structures important in controlling this righting response (Day et al., 2019). Further, bay scallops, Pecten fumatus, exposed to air gun noise had higher mortality rates, higher rates of recessing behavior, and changes in haemolymph biochemistry suggestive of reduced capacity for homeostasis (Day et al., 2017). These studies indicate diverse potential noise impacts on cephalopods and other invertebrates, though the extent of impacts on these taxa are only just beginning to be understood.

The current study focused on the longfin squid, Doryteuthis pealeii, which inhabits continental shelf waters in the Western Atlantic Ocean, ranging from Venezuela to Newfoundland. The species is most abundant in the Northeast U.S., between Cape Hatteras, NC, and Georges Bank (Hanlon et al., 2013). In that region, offshore wind farms are planned for construction in the 2020s and 2030s within 18 established lease areas (Musial et al., 2020). Longfin squid are commercially important in the United States, with average annual landings of about 11,000 mt and values of $\$ 30$ million since 2010 (NMFS, 2021). They are opportunistic 
predators that feed on a wide variety of fish and invertebrate species throughout their lifetime (Hunsicker \& Essington, 2006; Vovk, 1985). Small, young juveniles feed primarily on copepods, and they consume increasingly larger fish prey as they grow into adults. Squid rely heavily on visual cues for communication and finding prey, and are more likely to pursue mobile prey than stationary prey (Hanlon \& Messenger, 2018). Longfin squid are known to feed during the daytime and at night (Macy, 1982; Vovk, 1985). They have fast metabolisms, rapid digestion rates, and limited energy stores; thus it is suspected they need to frequently consume prey to survive in the wild (Hanlon et al., 2013; Hatfield et al., 2001).

In the present study, we examined how playbacks of sounds from impact pile driving influenced predation by the ecologically key squid $D$. pealeii. In both daytime and nighttime trials, live killifish, Fundulus heteroclitus, were added to the experimental tank to quantify squids' prey capture rates, failed predation attempts, and latencies to predation behaviors. We also quantified the mobility level of the killifish as a potential covariate in squid feeding behaviors, and we measured the hearing range of $F$. heteroclitus using neurophysiological auditory evoked potential (AEP) methods to assess their ability to detect the sounds. This study intends to elucidate how pile driving noise may alter feeding behaviors critical for individual squid's survival.

\section{METHODS}

Experiments during the daytime ("Day" trials) were conducted between June 23 and July 27, 2018 ( $n=54$ trials). Experiments during the nighttime ("Night" trials) were conducted between September 4 and October 21, 2018 ( $n=32$ trials). Day trials took place during daylight (between 09:00 and 18:45 local time), and Night trials took place after astronomical twilight (between 20:00 and 02:45 local time).

\section{Animal collection and care}

Squid were collected from Vineyard Sound $\left(41^{\circ} 22^{\prime} \mathrm{N} ; 70^{\circ} 47^{\prime} \mathrm{W}\right)$ via trawls conducted by the Marine Biological Laboratory (Woods Hole, MA). Recently trawled squid were 
transported in seawater-filled coolers to flowing-seawater facilities at Woods Hole Oceanographic Institution (WHOI; Woods Hole, MA). At WHOI, prior to experiments squid were held for 1-4 days in a semi-outdoor tented building in cylindrical holding tanks at least $1.2 \mathrm{~m}$ in diameter and with $0.8 \mathrm{~m}$ water depth $(900 \mathrm{~L})$ with ambient flowing seawater. The water temperature of these holding tanks was $21.7 \pm 0.9^{\circ} \mathrm{C}$ (mean \pm SD) from June to July and $19.0 \pm$ $1.5^{\circ} \mathrm{C}$ from September to October, and they were subject to the natural light cycle. Squid sharing a tank were same-sex and of similar size, to minimize damage to squid due to aggression, and densities were kept no higher than one squid per 225 L. Squid were fed every evening (16:00-19:00 local time) with killifish, Fundulus sp., (WHOI IACUC approved) collected from local estuaries.

\section{Experimental tank setup}

Experiments took place in a $1.1 \mathrm{~m}$ diameter cylindrical tank, filled to $0.5 \mathrm{~m}$ depth with ambient flowing seawater (Fig. 1). An Aqua-30 speaker (Theunissen Technical Trading, Malden, The Netherlands; frequency response: $100 \mathrm{~Hz}-10 \mathrm{kHz}$ ) was suspended, facing horizontally, at 25 $\mathrm{cm}$ depth and $15 \mathrm{~cm}$ forward of the closest tank wall. The speaker was connected to a PLA-2378 amplifier (Pyle Audio, Brooklyn, NY) powered by a $12 \mathrm{~V}$ battery. Audio files were played from a laptop connected to the amplifier. To monitor ambient tank sounds and noise playbacks during experiments, a hydrophone (HTI-96-MIN; High-Tech Inc., Long Beach, MS; frequency response: $2 \mathrm{~Hz}-30 \mathrm{kHz}$ ) was placed $2 \mathrm{~cm}$ from the tank wall, $44 \mathrm{~cm}$ horizontally from the speaker, and 35 $\mathrm{cm}$ deep. This hydrophone was attached either to a SongMeter 2 (Wildlife Acoustics, Maynard, MA) or (due to equipment failure) a SoundTrap ST4300 (Ocean Instruments, New Zealand). An overhead Sony video camera (for Day trials: model HDR-CX440; for Night trials: model HDRXR550) was used for all video analyses. A small PVC pipe cap with mesh over its bottom opening was used to hold an individual killifish in the tank until the experiment began. A rope was attached to the cap so the experimenter, out of view of the squid, could pull the cap up to reveal the fish. The water temperature of the experimental tank was $22.1 \pm 0.7^{\circ} \mathrm{C}$ for Day trials and, due to technical difficulties in efforts to heat tank water, temperatures were lower for Night trials: $16.1 \pm 1.9{ }^{\circ} \mathrm{C}($ mean $\pm S D)$. Squid generally increase their feeding rate and energy 
consumption with increased temperature, and feeding rates may be doubled with an increase in $10^{\circ} \mathrm{C}$, at least when prey are readily available (Boyle \& Rodhouse, 2005; O’Dor et al., 1980). Additionally, feeding habits of wild longfin squid may vary seasonally (Macy, 1982; Vovk, 1985). Due to the temperature and seasonal differences of Night trials from Day trials, Night trials were analyzed separately from Day trials, and comparisons between the two datasets are not made. Differences between holding tank temperatures and experimental tank temperatures at the time squid were transferred between these tanks were $0.6 \pm 0.6{ }^{\circ} \mathrm{C}$ for Day trials, and $3.0 \pm$ $1.9{ }^{\circ} \mathrm{C}$ for Night trials.

For Night trials only, black tarps were hung around the experimental tank to block out lights from playback equipment and the video monitor, and a U6R infrared light (Univivi, China; $850 \mathrm{~nm}$ wavelength) above the tank provided illumination for the camera, which was set to 'night mode' (similar to York et al., 2016). Visual photoreceptors of $D$. pealeii contain only one pigment, which has peak sensitivity at $493 \mathrm{~nm}$ (Hara \& Hara, 1976), well below the wavelength of the infrared light.

\section{Audio playback files}

Audio files of pile driving sounds used for the experiments were recorded during construction of the Block Island Wind Farm (BIWF, Rhode Island, USA) on October 25 ${ }^{\text {th }}, 2015$, at 19:38 UTC, from a hydrophone (HTI-94-SSQ, High Tech Inc., sensitivity: $-203.8 \mathrm{~dB}$ re $1 \mathrm{~V} / \mu \mathrm{Pa}$, gain: $6 \mathrm{~dB}$, flat frequency response from $2 \mathrm{~Hz}$ to $30 \mathrm{kHz}$ ) on a benthic sled $26 \mathrm{~m}$ deep and $500 \mathrm{~m}$ away from a pile driving site, and about $1 \mathrm{~m}$ above the seabed (Amaral et al., 2018). Files were recorded at a $9766 \mathrm{~Hz}$ sample rate. These data were provided to the authors (see Acknowledgements). The time interval between pile strikes was $1.8 \mathrm{~s}$. The steel, hollow pile had a diameter of $127.0 \mathrm{~cm}$, wall thickness of $3.8 \mathrm{~cm}$, a rake of $13.27^{\circ}$ with respect to vertical, and was driven up to $76.2 \mathrm{~m}$ deep into the seabed.

To avoid pseudoreplication of playback stimuli, three 10-min long recordings were generated from one pile driving bout and edited in Adobe Audition (Adobe Systems, San Jose, CA) prior to playback. The files consisted of a 1-min sequence of pile strikes with a randomized order of pile strikes, and this sequence was repeated for a total file length of $10 \mathrm{~min}$. The 
intervals between all pulses were edited to be $2 \mathrm{~s}$ long. The recordings were amplified by a custom magnitude to obtain the highest playback sound levels possible without clipping, with the goal to match received sound pressure levels in the tank with those present $0.5 \mathrm{~km}$ from the BIWF pile driving site, i.e. 190-194 dB re $1 \mu \mathrm{Pa}$ (zero-to-peak). For Control trials, a 10-min long silent file was played, to account for potential influences of the powered playback system's electrical field.

\section{Experimental procedure}

Only healthy squid (i.e., those without large skin lesions and with normal feeding behavior, having eaten a killifish the prior day) were selected for experiments (mantle length $8.5-23.0 \mathrm{~cm}$, mean $\pm S D: 15.5 \pm 3.0 \mathrm{~cm}$ ). At the start of an experiment on a given squid, approximately $24 \mathrm{~h}$ had elapsed since the squid last ate, so they were more likely motivated to feed during the experiment (Bidder, 1950). Each trial used a different squid; individuals were not retested. At the start of a trial, a killifish (total length $3.5-8.0 \mathrm{~cm}$, mean \pm SD: $5.2 \pm 1.2 \mathrm{~cm}$ ) was placed in the PVC cap in the experimental tank prior to adding the squid. The range in body length of prey that squid consume positively correlates with squid mantle length (Vovk, 1985), and we took care to scale the length of killifish added with squid length for each trial. Hydrophone and video recording began just after squid were transferred to the experimental tank. Squid were acclimated to the tank for at least 10 min prior to starting an experiment, until squid were consistently swimming back-and-forth in the middle of the tank and not interacting with the tank walls. As determined in prior studies, $10 \mathrm{~min}$ was sufficient time for these squid to return to this 'normal' in-tank behavior after transfer to a new tank (Jones et al., 2020; Mooney, Samson, et al., 2016).

There were three playback treatment types, designated "Onset", "5 min", and "Control". Treatment was randomly selected for each trial. In Onset trials, the experimenter raised the PVC cap to reveal the killifish prey after the acclimation period, and waited for the squid to start pursuing it, at which time the pile driving noise was immediately started. The noise exposure lasted for 10 min or until the squid captured and began consuming the fish. In $5 \mathrm{~min}$ trials, the pile driving playback was started after the acclimation period but five minutes before the fish 
was revealed. After revealing the fish, the playback continued for five more minutes or until the squid captured and began consuming the fish. Control trials had the same protocol as Onset trials, except that a 10-min long silent file was played instead of the pile driving noise file. For all trials, if the squid did not pursue the fish within $10 \mathrm{~min}$ after the fish was revealed, the trial was ended. The noise exposure duration was chosen based on observations of squid in preliminary noise trials that consumed prey less than $10 \mathrm{~min}$, often within $1 \mathrm{~min}$, after its reveal. Though durations of individual pile driving periods are variable in the field, this experimental duration was within the range of those observed for BIWF construction (Amaral et al., 2018). A summary of sample sizes is presented in Table 1.

\section{Acoustic calibration of the experimental tank}

Recordings of the sound field in the experimental tank followed methods and instrumentation used in Jones et al. (2020), and are briefly described here. The experimental tank was calibrated in $10 \mathrm{~cm}$ increments in all three dimensions ( 280 positions total) without animals present, creating a 3D array of received sound levels. The same noise file (looped in experiments) was played for $1 \mathrm{~min}$, with the same equipment as in experiments. Cephalopods are thought to detect acoustic particle motion rather than pressure (Budelmann, 1992b), and particle acceleration is likely the relevant transduction stimulus (Budelmann \& Tu, 1997). Particle acceleration in the tank was recorded with a PCB triaxial accelerometer (model W356B11; frequency response: $0.5 \mathrm{~Hz}-5 \mathrm{kHz}$, and sound pressure was recorded with a Reson TC4013 hydrophone (frequency response: $1 \mathrm{~Hz}-170 \mathrm{kHz}$ ) for ease of comparison with other studies.

\section{Acoustic data analyses}

Acoustic data analyses were conducted following methods described in detail in Chapter 2. Briefly, zero-to-peak levels of individual pile impulses were calculated, in $\mathrm{dB}$ for sound pressure $\left(\mathrm{SPL}_{\mathrm{z}-\mathrm{pk}}\right)$ and particle acceleration $\left(\mathrm{SAL}_{\mathrm{z}-\mathrm{pk}}\right)$ as follows:

$$
S P L_{z-p k} \text { or } S A L_{z-p k}=20 * \log _{10}\left(X_{p k}\right)
$$


where $X_{p k}$ is the maximum absolute $\mu \mathrm{Pa}$ or $\mu \mathrm{m} \mathrm{s}^{-2}$ (identical to Equation 3, Chapter 2). For simplicity, the 3D norm of particle acceleration (a vector quantity) was calculated and its magnitude is reported. Power spectral density (PSD) was calculated as in Chapter 2. All acoustic analyses were limited to a $20-1000 \mathrm{~Hz}$ frequency range.

\section{Behavior data analyses}

Squid predation sequences followed four stages categorized, in order of occurrence, as orient, pursuit, attack, and capture. 'Orient' is when the squid turns its body to face the prey head-on, 'pursuit' is when the squid swims head-first toward the prey, 'attack' is when the squid extends its tentacles in an attempt to capture the prey, and 'capture' is when the squid successfully catches the prey then brings the prey to its beak for ingestion.

Squid feeding behaviors were classified into three groups: 'no attempt', if the squid made no predation attempt (no pursuit or attack behaviors); 'failed attempt', if squid had at least one failed predation attempt (a pursuit or attack that did not result in successful fish capture) and no subsequent capture; and 'capture', if the squid successfully captured and began ingesting the fish (some of these squid had failed attempts beforehand). One squid in the Day Control treatment captured then immediately released the fish within $1 \mathrm{~s}$, and did not recapture the fish during the trial, thus was placed in the 'failed attempt' category. To compare proportions of squid with these outcomes across treatment types, 2x2 Fisher's Exact tests were performed (expected counts were too low to perform chi-square tests). Importantly, by experimental design, when limiting analyses to trials only in which a playback file was started, no squid in the Control and Onset treatments could belong to the 'no attempt' category since playback was only started if the squid pursued the fish. The proportion of squid that made no attempt to capture the fish in $5 \mathrm{~min}$ trials may include squid that were not motivated to feed during the time of experimentation, i.e., they may have similarly not pursued the fish if they were tested in Onset or Control trials. Therefore, statistical comparisons of capture rates among trials with audio playback were made only between Control and Onset treatments.

The number of failed predation attempts was also tracked, among a subset of trials that met two criteria: 1) a silent or pile driving playback was started, and 2) the squid made at least 
one predation attempt. Squid that captured the fish on their first predation sequence were assigned a failed attempt count of zero. These data were not normally distributed, thus KruskalWallis tests and pairwise Mann-Whitney $U(M W U)$ tests were used to compare the median number of failed predation attempts across treatment types. The time from when the killifish prey was revealed to when the squid first exhibited each of the four predation sequence stages (orient, pursuit, attack, capture) was quantified, hereafter referred to as 'predation latency'. (quantified separately for each stage). Statistical differences for predation latency were tested across treatment types with Kruskal-Wallis tests. Due to lower sample sizes for the 'Night' dataset, this analysis was only performed on the 'Day' dataset.

Locomotion of killifish during trials was quantified as the proportion of time the killifish spent swimming, and analyzed as a potential covariate of predation latency. 'Swimming' was defined as translational movement, as opposed to finning or pivoting in one location. Killifish swimming was quantified in $2 \mathrm{~s}$ time bins from the time the fish was revealed to the time it was captured, or until five minutes elapsed, whichever came first. These data were log-transformed to reach an approximately normal distribution, for use in linear regression analyses and analysis-of-covariance (ANCOVA). Again, due to lower sample sizes for the 'Night' dataset, this analysis was only performed on the 'Day' dataset.

Squid alarm responses, including inking, jetting, startle responses, and body pattern changes were also quantified. These behaviors were depicted and their ecological functions described in detail in Hanlon et al. (1999); criteria for their classification can be found in Jones et al. (2020). We chose to focus on alarm responses naturally employed by squid as antipredator defense behaviors. Inking refers to the release of ink, jetting is a fast, backwards propulsive escape response, and startle responses are sudden locomotor movements other than jetting. Body pattern changes are changes in the color and pattern of a squid's skin via specialized organs (chromatophores) and cells (e.g. iridophores). Body pattern changes are expressed to startle, bluff, or distract predators, and to communicate with conspecifics. We sought to compare alarm response rates when squid were hunting killifish at the start of noise (Onset trials) to those rates when the killifish was hidden from the squid ( $5 \mathrm{~min}$ trials) to see if the presence of prey and active hunting behavior altered these response rates. These analyses 
were similar to those from a prior study analyzing alarm responses in the same experimental setup but not in a feeding context (Jones et al., 2020). In that previous study, nearly all alarm responses occurred during the first minute of playback. Thus, analyses of alarm behaviors during playback periods in the present study focused on the first minute of playback. Inking, startle behaviors, and body pattern changes were difficult to observe from video in Night trials, thus most analyses of alarm responses were limited to Day trials, and only jetting was analyzed in Night trials. Alarm response rates were quantified and compared in three ways: 1) between the last minute preceding the start of noise or Control playbacks (pre-playback period) and the first minute of the playback period (Fisher's Exact tests), 2) during the first minute of playback among the three playback treatment types (Fisher's Exact tests), and 3) per pile impulse (quasipoisson GLMs).

Whenever multiple pairwise tests (either Fisher's Exact or MWU) were performed to compare playback treatments (three comparisons), Holm's sequential procedure was used to determine significance thresholds $(\alpha)$. Holm's is a modification of the Bonferroni procedure, and is just as effective at controlling for type I error, but reduces the likelihood of type II error (Eichstaedt et al., 2013). Briefly, this involves first performing each comparison, then ordering resulting $\mathrm{p}$ values from smallest to largest. The comparison with the lowest $\mathrm{p}$ value is tested with a Bonferroni adjustment for all other comparisons, in our case at $\alpha=0.0167$. The comparison with the second lowest $\mathrm{p}$ value is tested with a Bonferroni adjustment for one fewer test, in our case at $\alpha=0.025$, and so on, so our third comparison is tested at $\alpha=0.05$. The procedure stops at the first non-rejection of the null hypothesis.

\section{Fundulus heteroclitus auditory evoked potentials (AEPS)}

The AEP method was used to determine the general hearing range of $F$. heteroclitus. This method records electrical responses of groups of auditory nuclei in the brainstem and eighth cranial nerve of fishes. Tonal stimuli are played to determine minimum response thresholds at multiple individual frequencies. The goal was to determine the species' hearing thresholds and range of sound sensitivity, and place these data in context with the frequency spectrum of pile driving noise played to killifish during experimentation. Killifish not used in 
feeding trials were used for these hearing tests $(n=6$, mean \pm SD of total length: $9.0 \pm 0.4 \mathrm{~cm})$. One additional, dead control fish (total length: $10.0 \mathrm{~cm}$ ) was measured to confirm responses recorded in live fish were of neural origin and not artifacts of the recording and playback system.

The experimental setup, recording, and calibration procedures were similar to those of (Stanley et al., 2020), and are described in detail in Appendix B (Supplementary Info). Briefly, fish were anesthetized in a dilute solution of $100 \%$ clove oil $(0.25 \mathrm{~mL}$ clove oil : $1 \mathrm{~L}$ seawater) to reduce large muscular movements. Fish were then placed in a $0.95 \times 0.6 \times 0.7 \mathrm{~m}$ (length, width, depth) PVC tank with a seawater temperature of $7.7 \pm 0.3^{\circ} \mathrm{C}$ (mean $\left.\pm \mathrm{SD}\right)$, and subdermal electrodes were inserted to measure neural responses. Auditory stimuli were generated using custom LABVIEW software (National Instruments) on a laptop (S6520 Lifebook S, Fujitsu), and were played through an underwater speaker (UW-30) in the tank. Stimuli were sinusoidal amplitude-modulated tone pips presented at $80,100,150,200,300,400,600$, and $800 \mathrm{~Hz}$, in random order. Stimuli were presented at a given frequency in decreasing amplitude until a response could no longer be seen in the recording waveform, then attenuated 5-10 dB further to ensure minimum response thresholds were reached. Auditory thresholds were determined by visual inspection of waveforms, whereby the lowest sound level at which a clear response was detected was taken as the threshold.

\section{RESULTS}

\section{Experimental acoustic field}

The confines of the tank provided a quiet and isolated background environment for the study. Pressure spectra of ambient sound in the tank were at least $30 \mathrm{~dB}$ lower than those of the pile driving noise, and spectral levels of the silent Control playbacks were similar to these ambient levels. Accelerometer recordings of ambient sound and silent playbacks resulted in flat spectra at the self-noise floor of the accelerometer, i.e. $55 \mathrm{~dB}$ re $1 \mu \mathrm{m} \mathrm{s}^{-2}$ (not shown), thus these conditions were likely at lower acceleration levels. 
$S A L_{z-p k}$ and $S P L_{z-p k}$ of pile driving playbacks were highly variable throughout the tank, ranging from about 130 to $150 \mathrm{~dB}$ re $1 \mu \mathrm{m} \mathrm{s}^{-2}$ and 160 to $180 \mathrm{~dB}$ re $1 \mu \mathrm{Pa}$, respectively (Fig. 2A). For both metrics, amplitudes were more variable in the horizontal plane than across depths, though generally, higher amplitudes were recorded at 20 and $30 \mathrm{~cm}$ depths than at 10 and 40 cm depths. Particle acceleration followed a complex, non-monotonic pattern with distance from the speaker. Sound pressure was higher closer to the speaker and dropped off with distance from the speaker along the $X$ and $Y$ axes, increasing again near the tank boundaries. Acceleration PSD in the tank exceeded that recorded in the field $(0.5 \mathrm{~km}$ from the Block Island Wind Farm), by up to $40 \mathrm{~dB}$ at frequencies below $400 \mathrm{~Hz}$ (Fig. 2B). Pressure spectra of noise pulses in the tank were generally lower than, and within $30 \mathrm{~dB}$ of PSD of the field recordings (Fig. 2C). Below $400 \mathrm{~Hz}$, the pressure spectra of pulses in the tank were closer to those in the field, generally within $20 \mathrm{~dB}$. The reader is referred to (Jones et al., 2019) for a more detailed description of these tank calibration data and acoustic propagation in the tank used for the present study.

Prey capture rates: Day trials

The proportion of squid that pursued or attacked fish without capture ("failed attempt") was greater in the Onset treatment, but not significantly so (Fig. 3A). Between Onset and Control treatments, there were no significant differences in rates of "failed attempt" (odds ratio $(O R)=0.25,95 \%$ confidence interval $(C I): 0.04-1.58, p=0.198)$ or 'capture' $(O R=4.06$, 95\% Cl: 0.63-26.1, $\mathrm{p}=0.198$; Fisher's Exact tests). A lower proportion of squid in the $5 \mathrm{~min}$ treatment captured the prey than in Control and Onset. In $5 \mathrm{~min}$ trials, some squid made no attempt to pursue or attack the fish ("no attempt"). Note that all squid reported in Fig. 3A were played a pile driving noise or silent Control file.

We sought to compare the proportion of squid that made no predation attempts in the 5 min treatment, with that of the other treatments (Fig. 3B). In this analysis, the "no attempt" category in the Control and Onset treatments defines squid that were likely not motivated to feed in the experiment tank, as these squid did not pursue the fish and were not exposed to any audio playback. Thus a similar "no attempt" proportion in 5 min treatments compared to 
the Control and Onset treatments suggests squid with "no attempt" in the 5min treatment were not motivated to feed prior to playback. Conversely, a higher "no attempt" proportion in the $5 \mathrm{~min}$ treatments suggests reduction in feeding behavior caused by noise playback. In Day trials, there was no significant difference in the proportion of squid that made "no attempt" in the $5 \mathrm{~min}$ treatment, compared to either Control (OR=0.16, 95\% $\mathrm{Cl}: 0.02-1.46, \mathrm{p}=0.104)$ or Onset (OR = 0.90, 95\% Cl: 0.21-3.66, $\mathrm{p}=1.000$; Fisher's Exact tests).

Prey capture rates: Night trials

In the "Night" dataset, there were similar rates of "failed attempt" or "capture" between Onset and Control treatments, though low sample sizes in these treatments, limited to the number of squid that pursued the fish, precluded our ability to perform statistical comparisons (Fig. 4A). Analyzing all Night trials performed, (as done for Day trials in Fig 3B), the "no attempt" proportion was similar (40-50\%) in Night Control and Night Onset treatments, and was higher (83\%) in the Night $5 \mathrm{~min}$ treatment than the control (though not significantly so: $p=0.074$, Fisher's Exact tests) (Fig. 4B).

\section{Failed predation attempts}

The median number of failed attempts was highest for squid in Onset trials (Fig. 5). There were significant differences among the three treatments $\left(\chi^{2}=8.08, d f=2, n=42, p=\right.$ 0.018; Kruskal-Wallis; Fig. 5). Pairwise tests revealed a significant difference in the median number of failed attempts between Onset and $5 \mathrm{~min}$ treatments $(z=2.43, U=138 ; p=0.015$; MWU with Holm's procedure; lowest $\mathrm{p}$ value of the three comparisons), and between Onset and Control treatments $(z=-2.36, U=50, p=0.018$; second lowest $p$ value). The failed attempt rate was statistically similar between squid in Control and 5 min treatments $(z=0.39, U=113, p$ $=0.697$ ).

In Day Onset trials, 53\% of failed attempts stopped at the pursuit stage, and the other $47 \%$ of failed attempts were missed attacks (15 total failed attempts). About $83 \%$ of failed attempts ended at the pursuit stage and $17 \%$ ended at the attack stage in Day 5 min trials (6 total failed attempts), and $43 \%$ and $43 \%$ in Control trials, respectively ( 7 total failed attempts). 
The remaining $14 \%$ in the Control trials represents one squid that captured then immediately released the fish.

\section{Predation latency}

Comparing the three playback trial types in Day trials, there were no significant differences in the time elapsed from when the fish was revealed to the squids' first display of each predation sequence behavior (orient, pursuit, attack, capture; $p>0.05$, Kruskal-Wallis tests; Fig. S1; detailed statistics in Table S1). Assessing the three playback treatments together, median latencies for orient, pursuit, attack, capture were 13, 15, 23, and 23 s respectively, with interquartile ranges of 3-37, 4-41, 9-80, and 11-100 s, respectively. Note that here, some sample sizes (see Fig. S1, Table S1) were smaller than those for analyses reported for Fig. 5 because not all squid exhibited all four predation sequence stages.

\section{Fundulus heteroclitus audiogram and activity levels}

Killifish responded to tones played at 80 to $400 \mathrm{~Hz}$ but did not respond to $600 \mathrm{~Hz}$ and $800 \mathrm{~Hz}$ tones (Fig. 6A). In terms of root-mean-square SPL, killifish had the most sensitive hearing (lowest thresholds) at $200 \mathrm{~Hz}$ and reduced sensitivities below and above $200 \mathrm{~Hz}$. Comparison with the frequency spectrum of in-tank pile driving noise playbacks from Fig. $2 \mathrm{C}$ indicates that killifish were able to detect the noise, at least at frequencies between 80 and $200 \mathrm{~Hz}$. For comparison, acceleration levels of pile driving noise during the experiment were close to or above squid hearing thresholds (reported in Mooney et al., 2010) from 100-300 Hz (Fig 6B).

Killifish mobility levels were correlated with squid predation latencies to address the hypothesis that squid would take more time to predate less mobile prey. The log-transformed time from fish reveal to the first occurrence of each of the four predation stages was negatively correlated with killifish activity levels (Fig. 7). There were significant correlations between prey mobility and log-transformed time to squids' first orient to, attack, and capture of the prey $\left(R^{2}=\right.$ $0.10,0.19,0.27$, and $p=0.044,0.010,0.003$, respectively). One-way ANCOVAs indicated that prey mobility was a significant covariate for the time to first attack ( $d f=1, F=7.60, p=0.010$ ), and capture $(d f=1, F=9.75, p=0.005)$, and not significant for the time to first orient $(d f=1, F$ 
$=4.09, p=0.051$ ) and pursuit $(d f=1, F=3.28, p=0.080)$. Neither playback treatment nor the interaction between playback treatment and prey mobility were significant factors in ANCOVAs, for latencies of any of the four predation stages ( $p>0.05$; detailed ANCOVA results in Table S1).

\section{Squid alarm responses}

We looked for potential effects of squids' engagement in hunting on their alarm responses to pile driving noise playbacks by comparing alarm response rates when the killifish was revealed and squid were pursuing it (Onset) to when the killifish was hidden (5min) at the beginning of playback. In Day Onset and Day $5 \mathrm{~min}$ treatments, there were larger proportions of each of the four alarm response types during the first minute of the playback period compared to the pre-playback period (Fig. 8). Proportions of 'no response' were higher in the pre-playback period compared to the first minute of playback. In the Day 5 min treatment, proportions of inking, jetting, startle, and body pattern change were significantly higher in the playback period $(p<0.001, p<0.001, p=0.007$, and $p=0.010$, respectively, Fisher's Exact tests; detailed statistics in Table S1). In Day Onset, only the proportion of jetting was significantly higher in the playback period $(p=0.002)$.

During the first minute of playback, a higher proportion of squid in Onset and $5 \mathrm{~min}$ treatments showed alarm responses compared to Controls, and a higher proportion of squid had no response in the Controls (Fig. S2). In Day trials, there was a significantly lower proportion of squid with 'no response' in the $5 \mathrm{~min}$ treatment compared to the Control treatment $(\mathrm{OR}=0.05,95 \% \mathrm{Cl}: 0.00-0.58, \mathrm{p}=0.015$, Fisher's Exact tests), and there were no significant differences in any response type between Onset and $5 \mathrm{~min}$ treatments. Pooling $5 \mathrm{~min}$ and Onset treatments and comparing them with Controls, there were significant differences in the proportions of inking $(O R=9.60,95 \% \mathrm{Cl}$ : 1.00-92.0, $\mathrm{p}=0.039)$, jetting $(\mathrm{OR}=10.50,95 \% \mathrm{Cl}$ : 1.56-70.76, $\mathrm{p}=0.016)$, and no response (OR =0.10, 95\% $\mathrm{Cl}: 0.01-0.67, \mathrm{p}=0.023$ ).

Alarm responses per pile driving impulse were analyzed for the first minute (30 impulses) of pile driving noise playback. The same patterns in alarm responses over time were observed in Day Onset trials, during which the squid was hunting at the start of playback, and Day $5 \mathrm{~min}$ trials, during which the fish had not yet been revealed (Fig. 9). Squid displayed alarm 
responses at the highest rates within the first 5 pile driving impulses. Inking behaviors were extinguished first, followed by jetting and startle behaviors, with body pattern changes persisting the longest. Quasipoisson GLMs indicated that pile impulse number was a significant predictor of each of the four alarm response types $(p<0.001)$, and that noise treatment was a significant predictor of inking behaviors ( $p<0.001$; higher rate for $5 \mathrm{~min}$ ), jetting behaviors $(p<0.05$; higher rate for Onset), and startle behaviors $(p<0.001)$, and not body pattern change behaviors ( $p>0.05$; detailed statistical results in Table S1). However, the low number of counts of inking behaviors and strong overlap in 95\% confidence regions (not shown) for GLMs of each alarm response type suggest similar initial response rates on the first impulse and similar rates of decreased response over time between $5 \mathrm{~min}$ and Onset trials. The reader is referred to Figure S3 for a comparison of these data, per pile impulse, to the 1 min period just preceding the start of pile driving noise playback.

\section{DISCUSSION}

Squid exposed to pile driving noise playbacks generally had lower prey capture rates, and squid were more likely to abandon pursuit of prey if noise started during their pursuit. Prey mobility significantly negatively correlated with squids' predation latency, whereas noise did not have a significant effect on predation latency. Squid exhibited similar alarm response rates during noise whether or not they were hunting at the start of noise. Together, these results suggest that pile driving noise seems to alter the feeding activity of squid and reduce squids' capacity to hunt. The extent or duration of this has yet to be addressed. Similar to the distracted prey hypothesis, noise may shift squid predators' attention away from feeding tasks and toward the noise, which, given the observed alarm responses, appears to be perceived as a threat.

In-tank acoustic levels relative to hearing thresholds and in-situ acoustics

Though in-tank particle acceleration levels of the pile driving noise playback were spatially variable, they remained near or above physiological hearing thresholds for longfin 
squid at some frequencies, notably around $100-200 \mathrm{~Hz}$. Along with the behavioral responses observed, this indicated squid were able to detect the noise playback. A comparison of sound pressure spectra of in-tank pile driving noise with killifish AEP data indicates that killifish were able to detect this noise at frequencies below $300 \mathrm{~Hz}$. In this frequency range, amplitudes of pile driving noise in the tank and in the field were higher compared to amplitudes at frequencies above $400 \mathrm{~Hz}$. Thus, as was the case with longfin squid, frequencies at which $F$. heteroclitus had highest sensitivities to sound overlapped with the frequency range at which pile driving noise tends to have highest energy.

Acceleration levels in the experimental tank exceeded those of the original file recorded in the field (Fig. 2). This result is to be expected; the ratio between particle motion and sound pressure is typically increased underwater in tanks, relative to deep-water, free-field conditions (Campbell et al., 2019; Rogers et al., 2016). Careful calibrations of pressure and particle motion, as done in the present study, are needed for tank studies to address the acoustic conditions of this complex environment. The higher particle acceleration levels in the tank relative to the field recording (500 $\mathrm{m}$ horizontally away from a pile and $1 \mathrm{~m}$ above the seabed) suggest that $S A L_{z-p k}$ in the tank correspond to water-borne $S A L_{z-p k}$ expected within $500 \mathrm{~m}$ from the BIWF pile driving operation (Amaral et al., 2018). Though acoustic propagation in tanks differs from that in the field, the present study allowed a controlled environment and presentation of sound, and detailed, individual-based quantification of behavior. The present study indicates that at least playbacks of pile driving noise appear to disrupt squid engaged in feeding behaviors, and serves as a stepping-stone to inform related, future noise exposure studies in field conditions.

\section{Prey capture rates}

There were overall trends of lower proportions of squid capturing prey in noise treatments compared to controls. Importantly however, lack of statistical significance in differences of these proportions prevents conclusive interpretations, and larger sample sizes would aid assessment of whether noise exposure significantly reduces the likelihood of squid attempting to capture and successfully capturing prey. Additionally, Fisher's Exact tests, which were used in our study to compare low-count proportion data, are potentially limited in 
statistical power (Lydersen et al., 2009). Though different conditions of Night trials (lower temperatures, fall season instead of summer) prevented us from assessing diel influences on feeding behavior during noise, variability in capture rates indicates the potential for diverse responses to noise under different environmental conditions.

\section{Failed predation attempts}

In the Onset treatment, squid had significantly more failed attempts compared to other playback treatments. This suggests that if a sudden onset of impulsive noise, such as pile driving, occurs while squid are actively feeding, squid are more likely to miss opportunities for prey capture. Notably, squid confined to the experiment tank readily had the opportunity for multiple capture attempts, always remaining in close proximity to the killifish prey which could not escape. Wild squid may not have additional chances at capturing a particular prey item, e.g., if squid abandon a predation attempt and prey escape. Such reductions in the success rate of prey capture could lead to lower squid survival particularly when squid might be more resource-limited, for example in winter months when squid have been found to have slower growth rates and a higher incidence of empty stomachs (Macy, 1982; Vovk, 1985). Squid in the family Loliginidae, including longfin squid, have relatively high metabolic rates, fast digestion rates (4-6 h), limited energy storage, and need to eat little at once, but often (Bidder, 1950; Boyle \& Rodhouse, 2005; Hanlon, 1990). Given this requirement of frequent feeding, if cessation of feeding during noise leads to longer-term reduced food intake, then the potential exists for population-level reductions in squid abundance. However, our study only addressed short-term impacts and we did not measure feeding behavior after noise exposure. As well, field pile driving operations occur for longer periods than in this study, up to several hours per day, though with variable noise (pile driving) and inter-noise (adjustment) periods (Amaral et al., 2018). Future studies should investigate chronic noise effects over longer exposures, and the potential for long-term habituation, with respect to feeding behaviors. Longer-term, comparative field studies will be necessary to address hypotheses regarding chronic, ecological, and population level effects. 


\section{Alarm responses in feeding vs. non-feeding contexts}

Squid that were hunting at the start of noise playback (Onset trials) and squid that had not yet encountered the fish at the start of noise playback ( 5 min trials) had similar alarm response rates to the first several noise impulses, and similar habituation rates over repeated impulses. Thus, the presence of a fish and a squid's engagement in feeding behavior did not influence the squid's alarm response rates during noise. Initial alarm responses and habituation rates to noise were similar to those in a prior experiment on solitary squids placed in the same tank, without any other animals (Jones et al., 2020). While squid show the potential to habituate to pile driving noise quickly, the fact that the onset of noise can disrupt a hunting sequence means that squids' hunting could be disrupted when pile driving suddenly commences, potentially causing it to lose a meal each time a hunt is interrupted. Indeed, the results of these alarm behavior analyses likely explain why the squid in the Onset trials exhibited significantly higher rates of failed prey capture attempts.

\section{Possible mechanisms driving behavior changes during noise}

There are several potential mechanisms for the observed increase in squids' failed predation attempts, which could be investigated in future studies. These include: 1) "distraction", or attention shifts, e.g. away from foraging behavior toward vigilance of potential predation threats, 2 ) increased stress which could arise via physiological changes and changes to behavioral motivation, 3) masking of hydrodynamic cues that might be utilized for detecting and accurately attacking prey, and 4) physical damage to squids' statocysts, which may detect acoustic cues, possibly including those resulting from prey movement.

Disruptions to foraging and feeding behaviors observed in the present study may be associated with "distraction", i.e. attention shifts toward threat stimuli. This is suggested by the fact that in most Onset trials with failed predation attempts, squid immediately ceased pursuit at the start of noise playback and simultaneously jetted away, sometimes inking as well. These are known natural defense behaviors employed in response to perceived predator threats, suggesting squids' attention was diverted from a feeding task and toward predator defense. Across multiple taxa, animals have been found to reduce their foraging activity in the presence 
of potential threat cues (i.e. cues suggesting a predation threat), including acoustic cues (Chan \& Blumstein, 2011; Dukas, 2002). For example, mud crabs (Panopeus spp.) in lab experiments significantly reduced their consumption rate of prey (clams) in the presence of played-back acoustic cues from predatory fish (Hughes et al., 2014). As evidenced by the occurrence of alarm responses in the present study, the attention shift hypothesis appears to be a likely candidate mechanism for squids' cessation of feeding behaviors during noise. However, the other potential mechanisms described below cannot be ruled out.

We did not measure physiological variables such as changes in stress hormones or changes in respiration rate, which have been found in several noise-exposure studies on crabs and fish (Purser et al., 2016; Putland et al., 2019; Simpson et al., 2015; Wale et al., 2013b). Thus, physiologically-induced stress cannot at present be excluded as a potential mechanism behind reduced feeding behavior during noise.

Squid appear to utilize hydrodynamic cues from swimming predators to avoid being captured (York \& Bartol, 2014; York et al., 2016), and, as suggested in cuttlefish (Komak et al., 2005), possibly utilize similar cues from prey to aid in making accurate attacks on prey. The presence of rows of hair cells (called the "lateral line analog") observed along the head and arms of cuttlefish and squid suggests that such a function may exist (Budelmann \& Bleckmann, 1988). Fish are known to utilize hydrodynamic cues detected by their lateral line in order to find prey (Coombs \& Braun, 2003). Although the ecological relevance of these available hydrodynamic cues to cephalopods remains unclear, water motion in noisy acoustic fields could in theory mask (i.e., prevent squids' detection and utilization of) hydrodynamic cues from prey, as has been suggested for fish (Mogdans, 2019). In the present study, this effect would be expected to contribute more to failed (i.e., missed) attacks (where the squid is closer to the fish and might receive stronger hydrodynamic cues), rather than the failed (i.e. abandoned) pursuits.

Some studies (e.g., Solé et al., 2017, 2018) have reported physical damage of sensory structures and hair cells in cephalopod statocysts and lateral line analogs after animals were exposed to long ( 2 h) continuous acoustic stimuli (sinusoidal frequency-modulated sweeps). Yet comparatively, impacts on auditory structures were limited or absent for fishes exposed to pile 
driving sounds, which were higher intensity than those used in the present study (Casper, Smith, et al., 2013). Indeed, though physical statocyst damage cannot be fully discounted, with the lack of comparative anatomical studies at shorter presentations ( $<10 \mathrm{~min}$ in this study), such damage may be less likely in our study.

\section{Comparisons with other taxa}

Only a handful of published studies have investigated influences of noise on invertebrate feeding behavior. A playback experiment with shore crabs (Carcinus maenas) found that if ship noise was played once crabs had found food and started feeding, they were more likely to cease feeding (Wale et al., 2013a). Similarly, squid in the present study often ceased pursuit of prey if noise was started during their predation sequence. Filter-feeding blue mussels (Mytilus edulis) increased their clearing rate during pile driving relative to ambient sound conditions, suggesting an increased feeding rate and metabolic demand during noise (Spiga et al., 2016). Mussels (M. edulis) have also responded to in-tank substrate vibrations by partially closing their valves, which could potentially lead to changes in food intake and respiration (Roberts et al., 2015). Natural noise (running water from a river) reduced maximum feeding rate and significantly reduced prey handling time in freshwater damselfly larvae (Ischnura elegans) feeding on Daphnia sp. (Villalobos et al., 2017). Thus, several studies have suggested negative effects of both anthropogenic and natural noise on feeding behaviors of aquatic invertebrates. Yet, noise impacts are only beginning to be investigated in these diverse taxa.

Few studies have investigated effects of noise on feeding and foraging behavior of fish as well. Because squid occupy similar trophic niches to many predatory fish (Boyle \& Rodhouse, 2005), such comparisons are useful to provide context, given the limited comparative cephalopod data available. Three-spined sticklebacks (Gasterosteus aculeatus) exposed to white noise had significantly higher food discrimination error and food-handling error when attempting to feed on Daphnia sp. prey (Purser \& Radford, 2011). Captive cichlids (Amatitlania nigrofasciata) significantly decreased foraging behavior, in terms of number of pecks and number of individuals foraging, during boat noise (McLaughlin \& Kunk, 2015). In a field-study, 
captive roach (Rutilus rutilus) and perch (Perca fluviatilis) made significantly fewer feeding attempts when exposed to noise from an actual boat motor (Magnhagen et al., 2017). Thus, like the present study with squid, multiple studies on fish have demonstrated reductions in feeding and foraging activity, and under a variety of noise conditions. These studies further demonstrate that a variety of variables pertaining to feeding behavior, such as quantity of food intake, capture rates, and time spent foraging, can be altered during noise, emphasizing the importance of quantifying multiple variables that may lead to changes in the amount or rate of energy intake.

\section{Conclusions and future directions}

The present study uniquely demonstrates how pile driving noise can alter the feeding behavior of squid. To the authors' knowledge, this study is the first to demonstrate changes in feeding behaviors of cephalopods during anthropogenic noise. These data underscore the importance of accounting for behavior of both predator and prey species, and for ecosystem dynamics, when assessing noise effects. Squid were significantly more likely to abandon pursuit of prey and have failed capture attempts when noise playback started during their predation sequences. In addition, a lower proportion of squid captured live killifish prey in noise exposure trials compared to silent Control trials, though these differences were not statistically significant. Missed opportunities for prey capture and lower feeding rates during anthropogenic noise could lead to reductions in growth or survival of individuals, particularly for longfin squid, with their high-metabolic rates that require frequent feeding; this could be especially damaging to squid survival when prey resources are limited. Future work should address potential longer term metabolic consequences of noise exposure. Squids' latency to capture prey was significantly negatively correlated with fish locomotion, emphasizing the importance of considering natural covariates at play when investigating effects of anthropogenic stressors on predator-prey relationships. Further, at the onset of noise exposure, when squid were engaged in hunting they had similar alarm response rates compared to when they were not hunting; this indicated that both in feeding and non-feeding contexts, individual squid were similarly alert to threat stimuli. Changes in feeding behaviors reported here have potential implications for 
reduced feeding activity of squid exposed to construction noise of marine pile driving operations. However, behaviors and acoustics observed in the laboratory may differ from those in situ. Thus, future comparative field studies are needed to further investigate influences of pile driving noise on foraging behaviors of squid. Further, the present results raise questions regarding how other key longfin squid behaviors such as breeding, shoaling, predator avoidance, and habitat selection may be impacted by noise. 
Table 1 Number of trials conducted for each of the six treatments. The first number is the total number of trials attempted, including both trials without playback and those with playback, and is the sample size for the analysis reported in Fig. 3B (Day) and Fig. 4B (Night). The second number (in parentheses) is the number of trials with playback, and is the sample size for analysis reported in Fig. 3A (Day) and Fig. 4A (Night). Results reported in Fig. 5, and Fig. 7-9 have sample sizes subset from numbers in parentheses here, according to conditions specified in their associated figure captions and text. Note that by experimental design, playback occurred in every ' $5 \mathrm{~min}$ ' trial, and playback was not started in Control or Onset trials if squid did not pursue the prey.

\begin{tabular}{lrrr}
\hline & Day & Night & Total \\
\hline Onset & $18(13)$ & $10(6)$ & $28(19)$ \\
5 min & $20(20)$ & $12(12)$ & $32(32)$ \\
Control & $16(15)$ & $10(5)$ & $26(20)$ \\
\hline Total & $54(48)$ & $32(23)$ & $86(71)$ \\
\hline
\end{tabular}




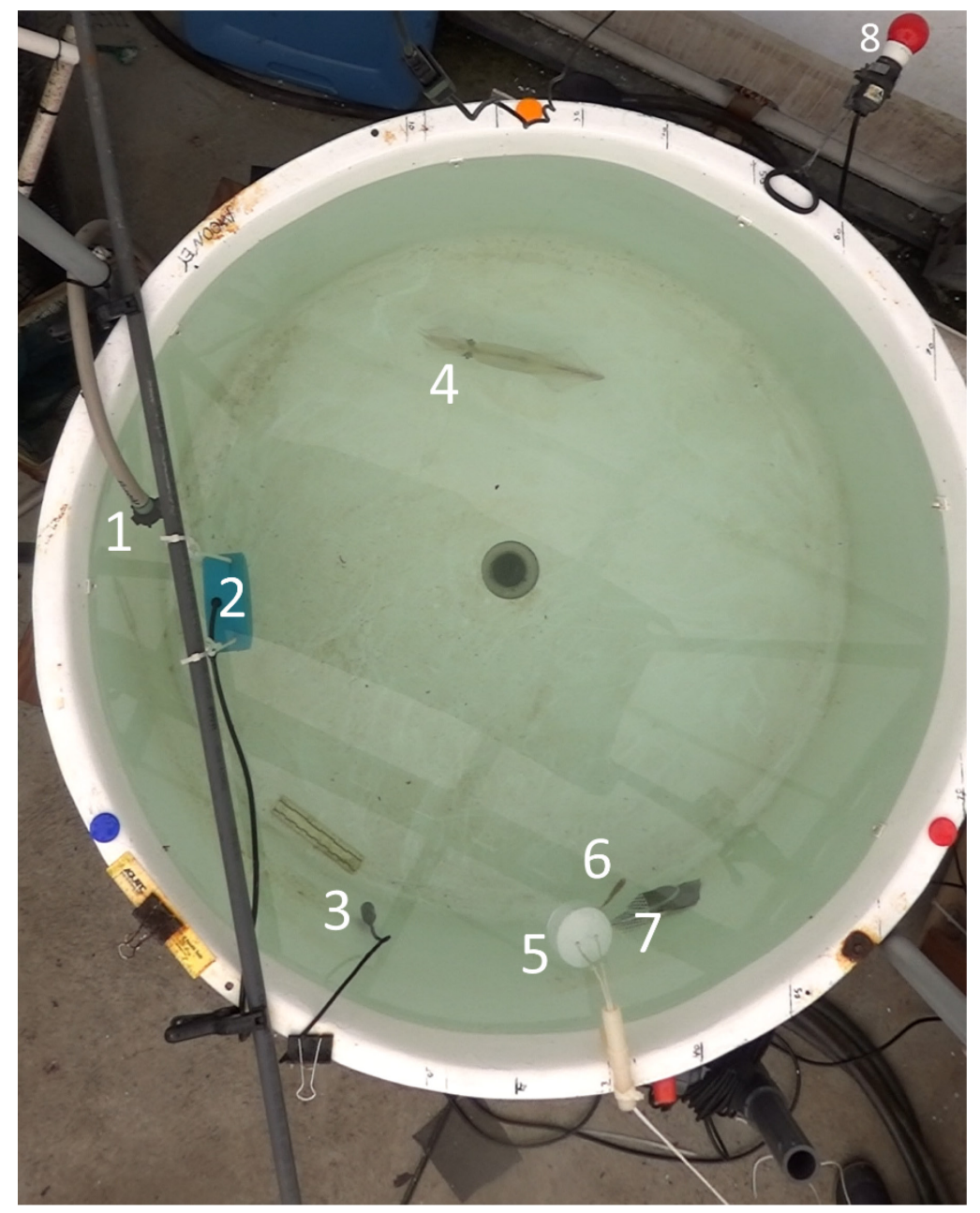

Figure 1. View of experiment tank from top-down video camera. 1) Inflow hose, 2) underwater speaker (Aqua-30), 3) hydrophone to monitor ambient sound and playbacks during experiments, 4) squid (Doryteuthis pealeii) 5) PVC container lifted to reveal fish, 6) fish prey (Fundulus heteroclitus), 7) flow outlet, covered with mesh, 8) LED to indicate start of playbacks (used only in Day trials). The dark circle in the center of the tank is a plugged outflow pipe 


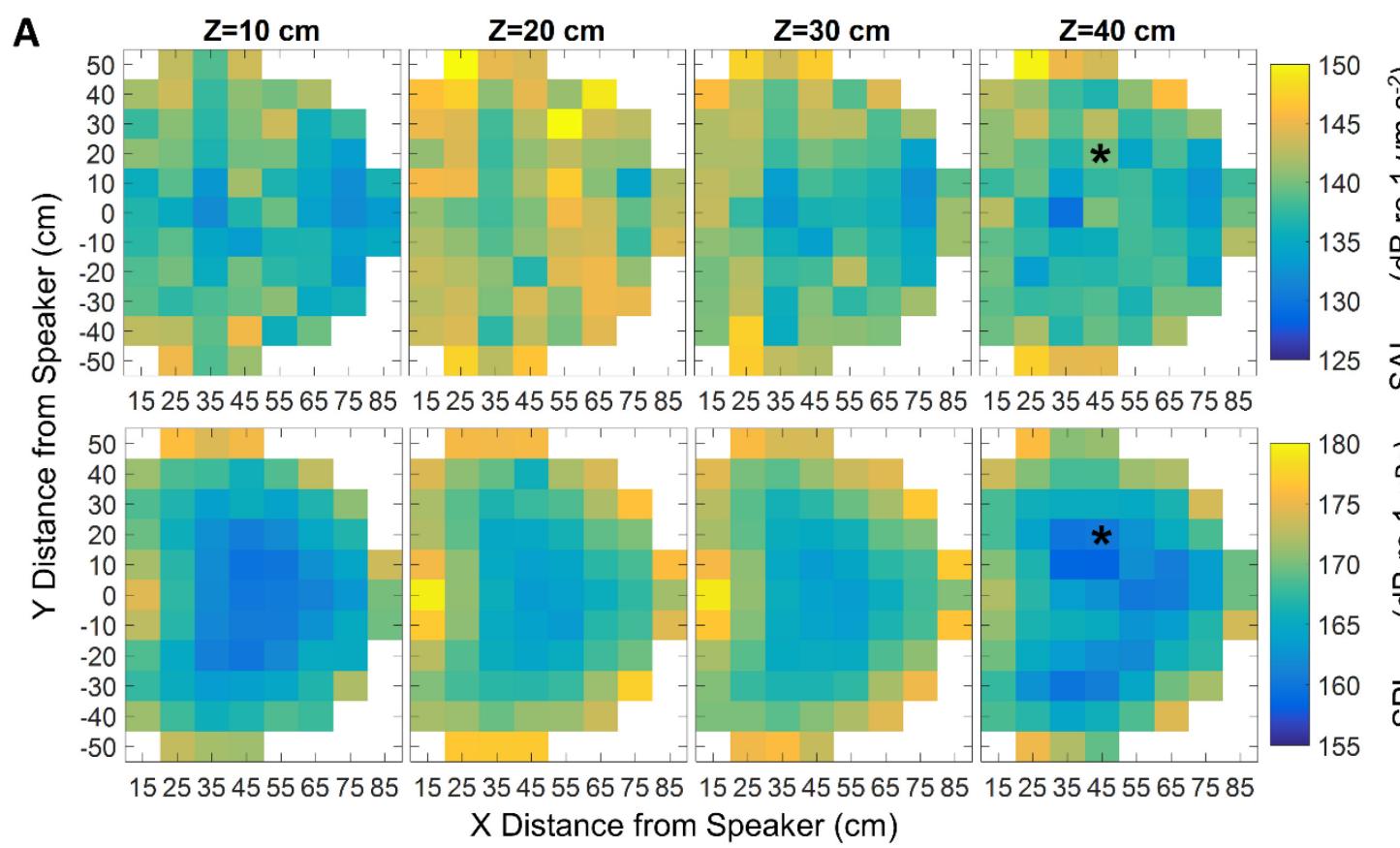

B

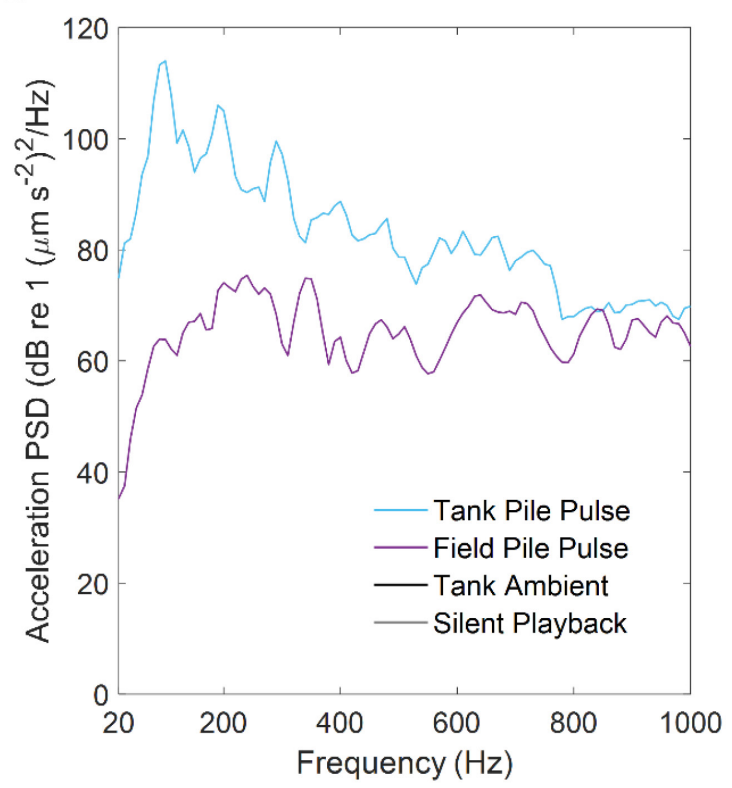

C

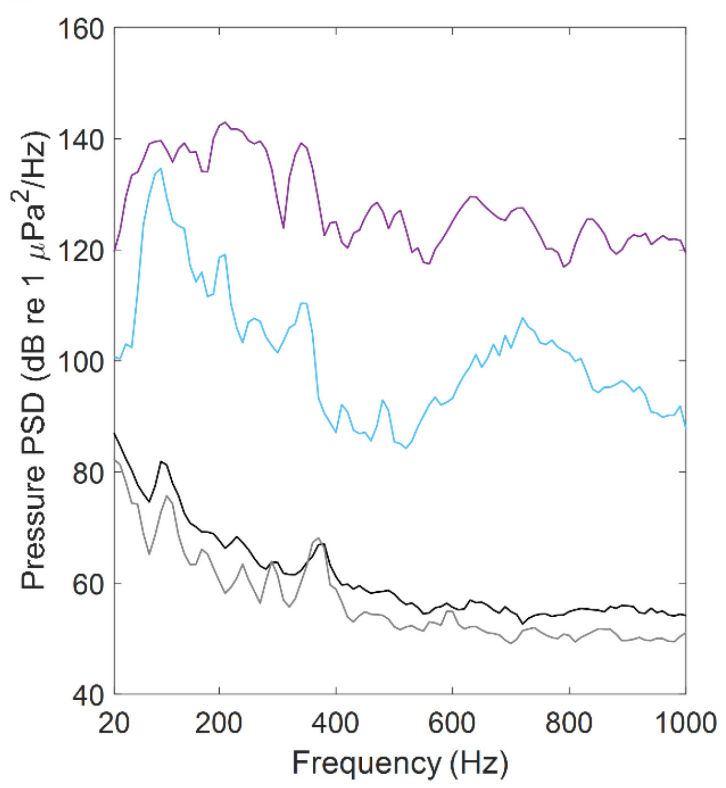

Figure 2. A) Spatial maps of zero-to-peak acceleration (top) and pressure (bottom) for four water depths (columns), shown from top-down in the horizontal plane, with the front-center of the speaker set as the origin. Data were band-pass filtered to $20-1000 \mathrm{~Hz}$ and median zero-to-peak values of pile pulses (across 30 pulses, i.e. $1 \mathrm{~min}$ ) are shown for each recording location. Asterisks indicate the recording location at which spectra are shown in B and C. Power spectral densities are shown for particle acceleration (B) and sound pressure $(\mathrm{C})$ in time windows covering pile driving pulses of in-tank playbacks and original field recordings. Median spectra of 30 pulses are shown. Spectra of ambient tank sounds (no playback) and the silent playback file are also shown in pressure, but not in acceleration since these conditions were below the noise floor of the accelerometer. 
A

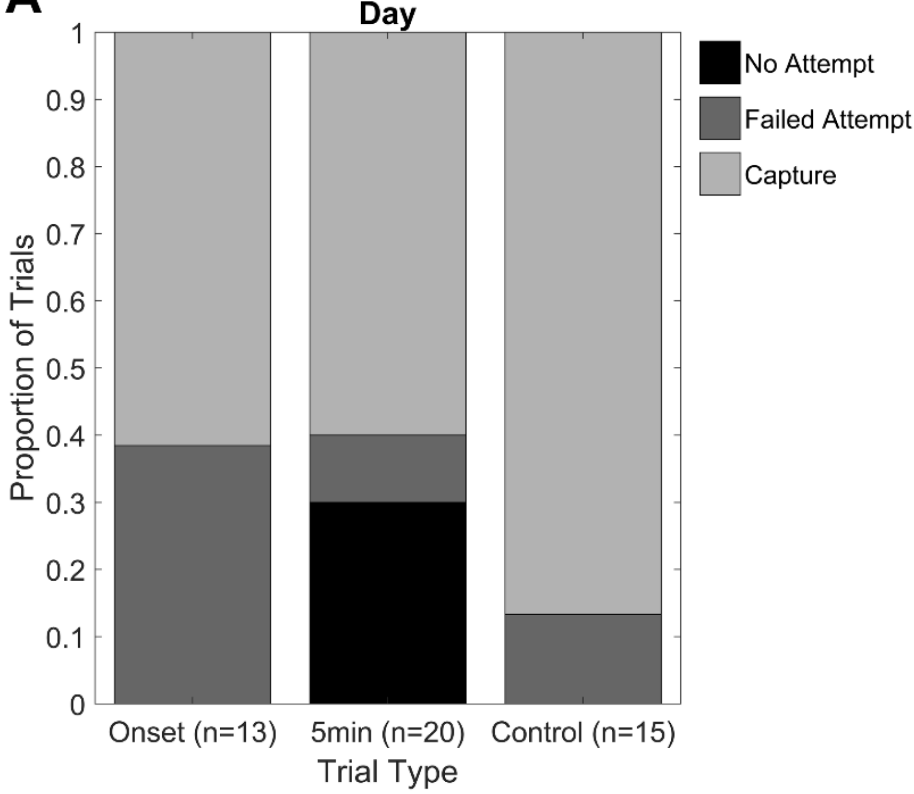

B

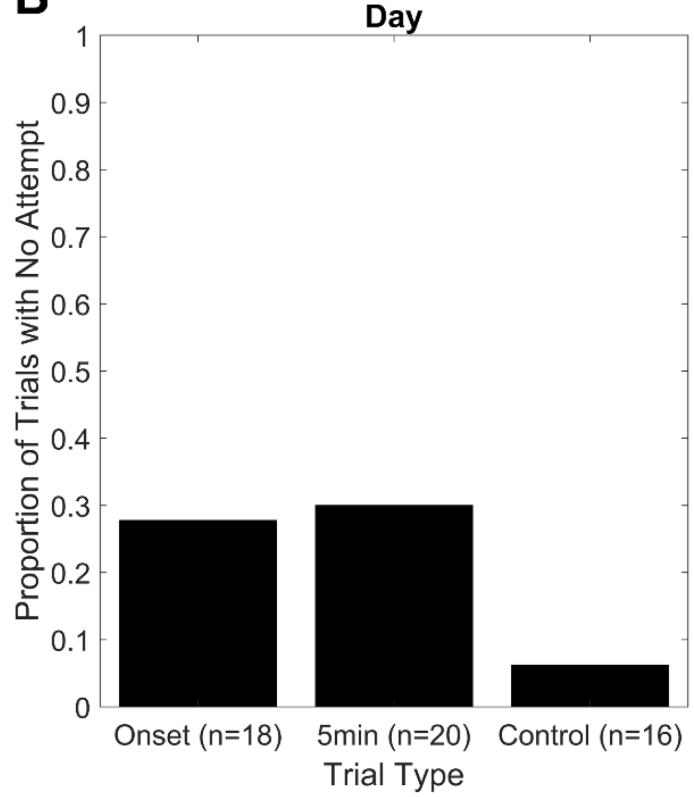

Figure 3. A) Proportions of trials in the 'Day' dataset, in which squid successfully captured and consumed the fish ('Capture'), attempted to capture (with pursuit and/or attack) but did not successfully capture ('Failed Attempt'), or made no attempt to pursue or capture the fish ('No Attempt') during playback, for each playback treatment. Only trials in which a silent or pile driving playback was started are included here. B) Proportions of squid that made no attempt to feed in the 'Day' dataset. Proportions of 'No Attempt' in Onset and Control treatments represent squid that received no noise exposure or control playback, respectively, as they did not pursue prey during the trial. Sample sizes for Onset and Control treatments are greater than in $3 \mathrm{~A}$ because trials in which no playback was started are included here. All squid in $5 \mathrm{~min}$ trials received noise exposure, starting $5 \mathrm{~min}$ before the prey was revealed. 

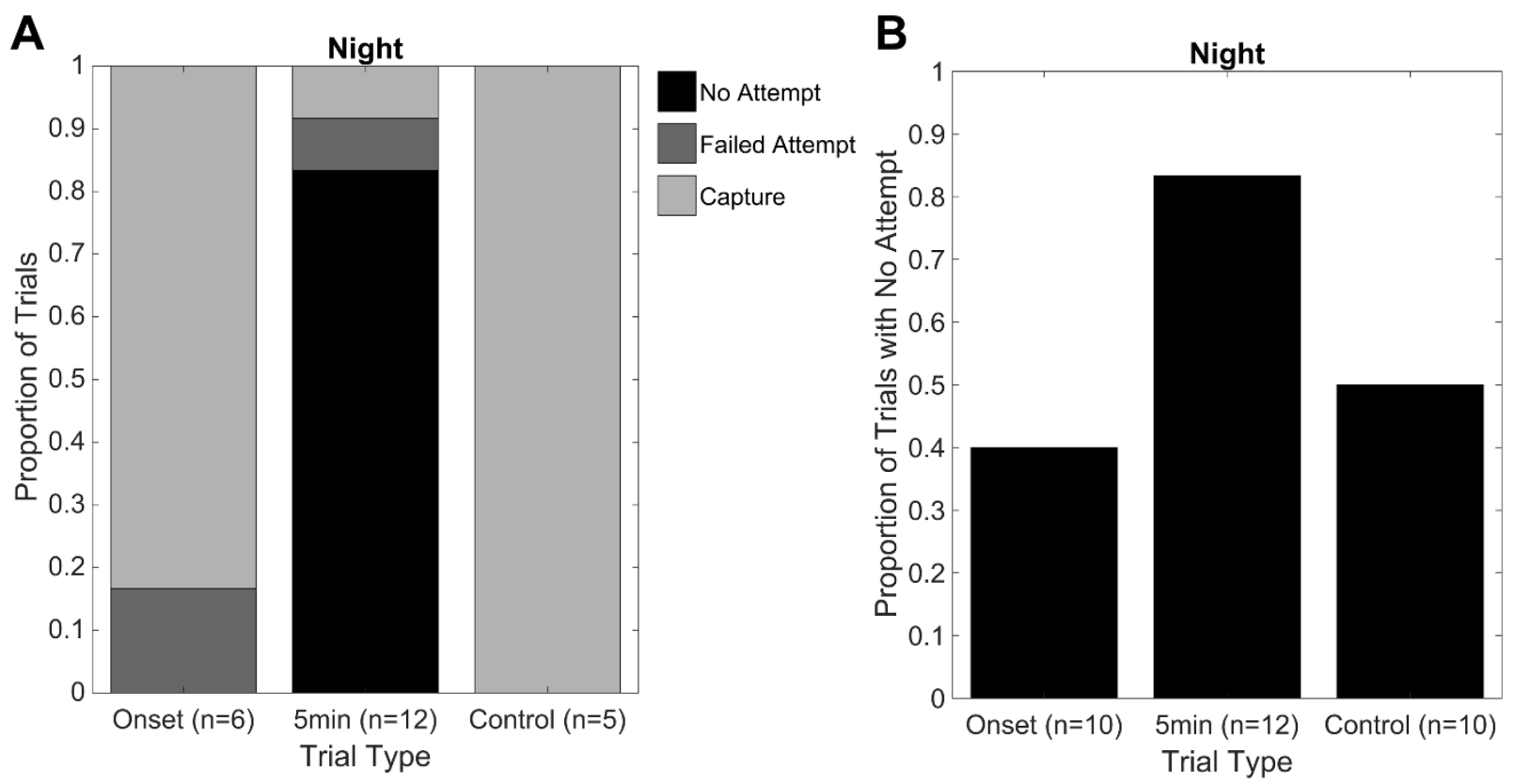

Figure 4. A) Proportions of trials in the 'Night' dataset for feeding behavior outcomes as detailed in Fig. 3A. Only trials in which a silent or pile driving playback was started are included here. B) Proportions of squid that made no attempt to feed in the 'Night' dataset. Proportions of 'No Attempt' in Onset and Control treatments represent squid that received no noise exposure or control playback, respectively, as they did not pursue prey during the trial. Sample sizes for Onset and Control treatments are greater than in Fig. 4A because trials in which no playback was started are included. All squid in $5 \mathrm{~min}$ trials received noise exposure, starting 5 min before the prey was revealed. 


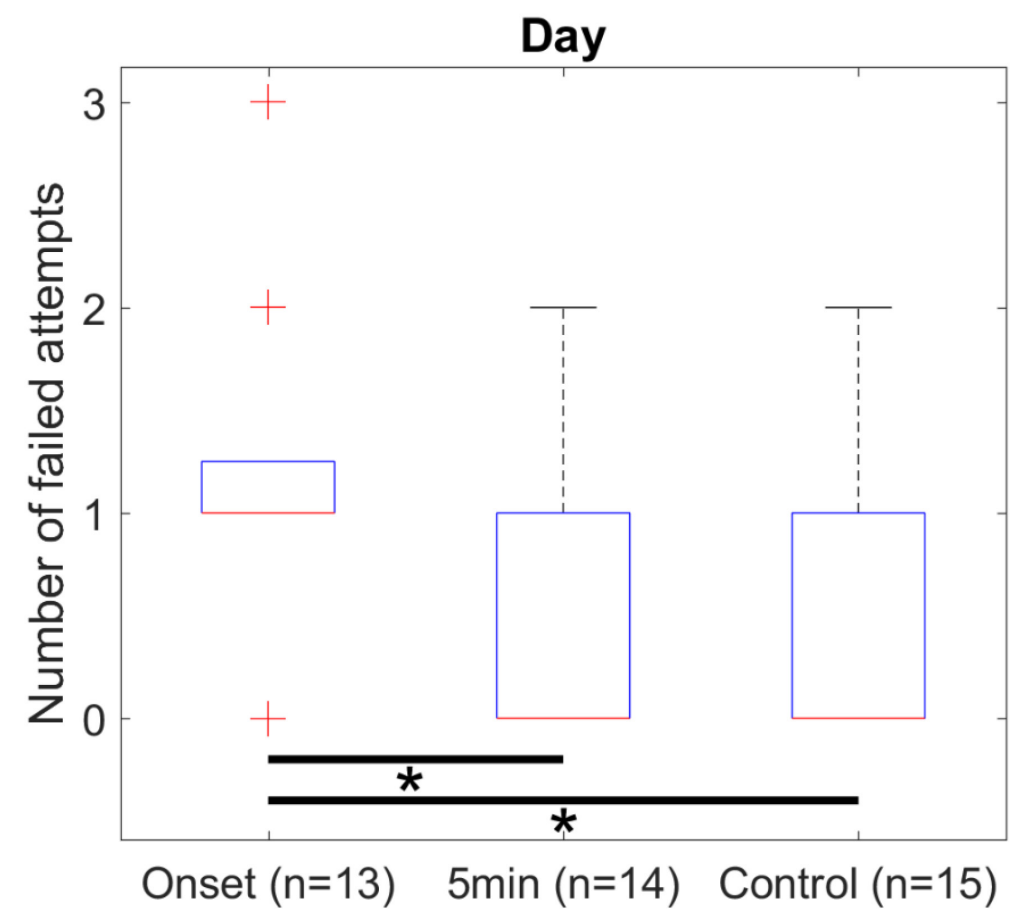

Figure 5. Number of failed predation attempts (defined as a pursuit and/or attack without capture during a predation sequence) for Day trials. Only trials in which a silent or pile playback was started are shown. Sample sizes are reduced here for the $5 \mathrm{~min}$ treatment compared to data presented in Fig. 3, because only trials in which squid made at least one predation attempt were included. Squid that captured the fish on their first predation sequence were assigned a failed attempt count of zero. Outliers (crosses) are defined outside the range $q_{3}+1.5 \times\left(q_{3}-q_{1}\right)$ and $q_{1}-1.5 \times\left(q_{3}-q_{1}\right)$, where $q_{1}$ and $q_{3}$ are $25^{\text {th }}$ and $75^{\text {th }}$ percentiles, respectively. Medians are at the bottom of each box, and whiskers extend to integer data points furthest from the median that are not outliers. ${ }^{*} p<0.017$ for Onset vs. 
A

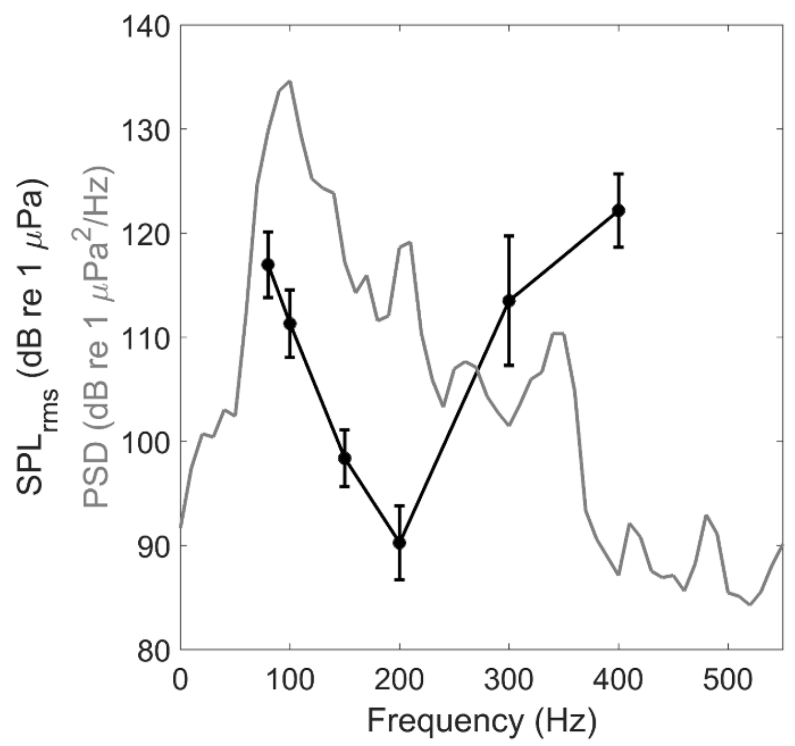

B

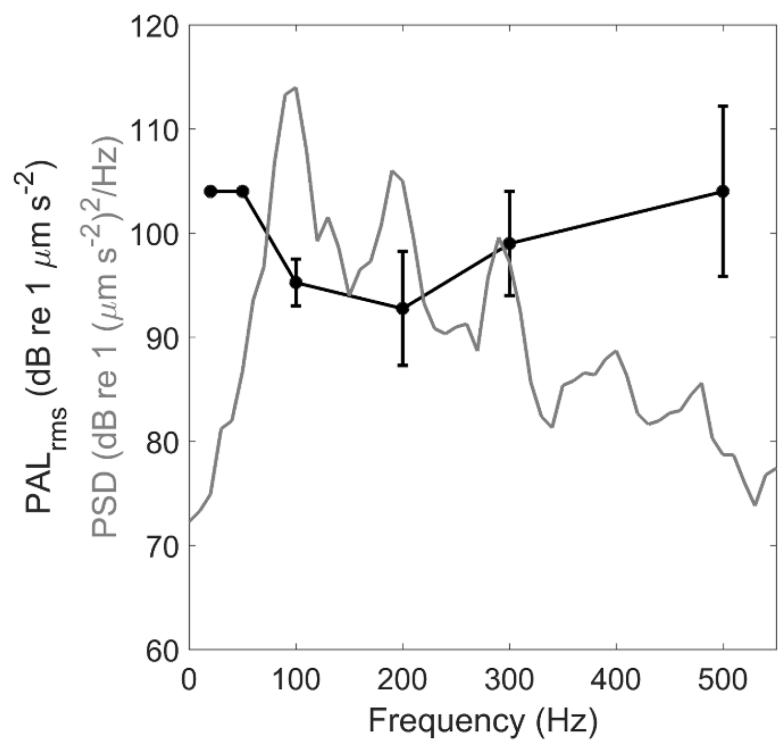

Figure 6. A) Black line: mean hearing thresholds of $F$. heteroclitus ( $n=6$ individuals) in terms of rootmean-square sound pressure levels at frequencies from $80-400 \mathrm{~Hz}$. No frequencies below $80 \mathrm{~Hz}$ were tested, and no responses above $400 \mathrm{~Hz}$ were detected (600 Hz and $800 \mathrm{~Hz}$ were tested). Grey line: pressure power spectral density (PSD) of pile driving noise in the experimental tank as shown in Fig. 2C. B) Black line: mean hearing thresholds of $D$. pealeii ( $\mathrm{n}=4$ individuals) in terms of root-mean-square particle acceleration levels at frequencies from $20-500 \mathrm{~Hz}$, adapted from Mooney et al. (2010). Grey line: particle acceleration PSD of pile driving noise in the experimental tank as shown in Fig. 2B. In A and $B$, error bars indicate standard deviation of the mean at each frequency. 

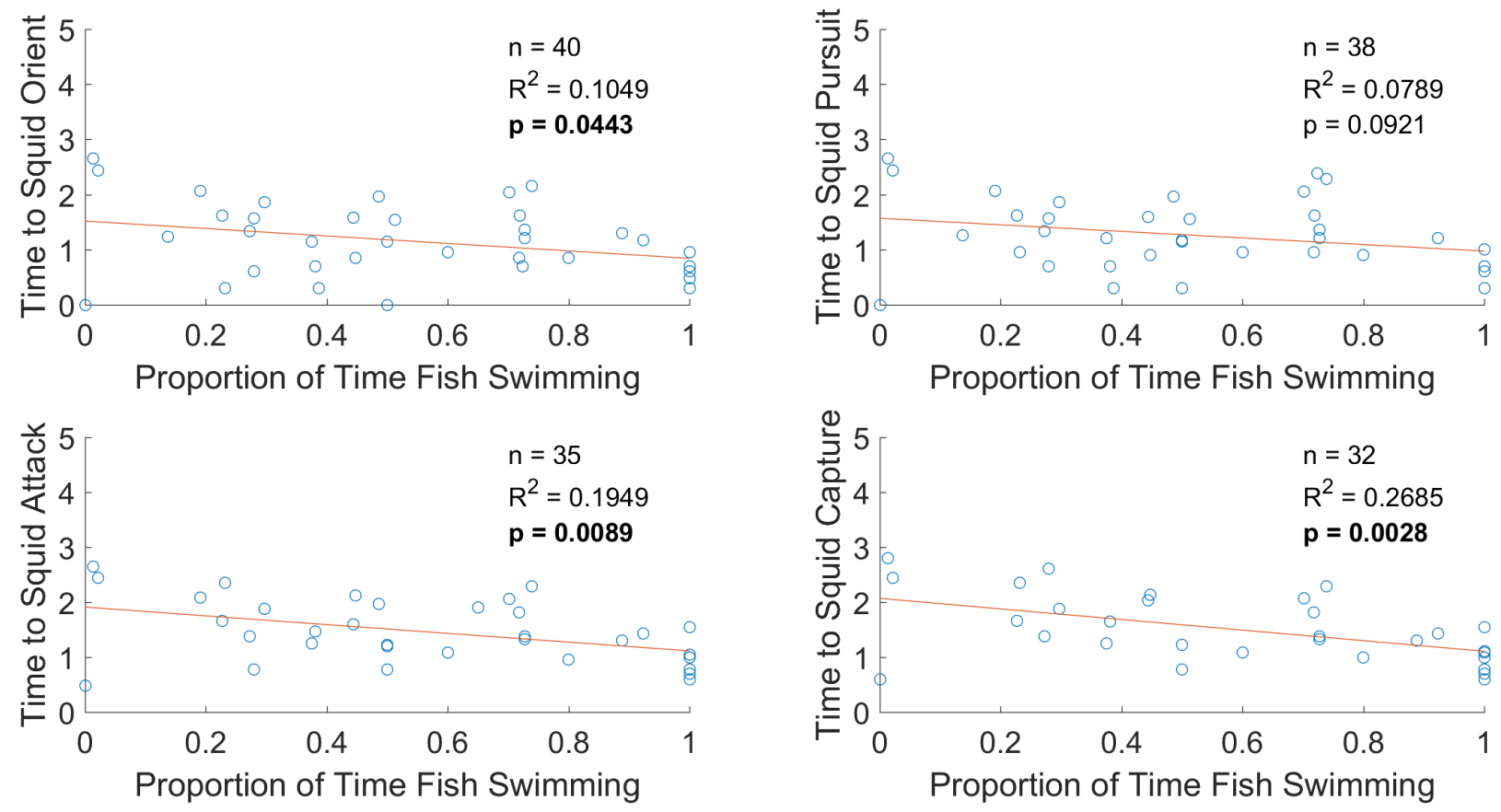

Figure 7. Log-transformed time from fish reveal to the first occurrence of four squid predation behaviors (orient, pursuit, attack, and capture) plotted against the proportion of time the fish spent swimming prior to the predation behavior or the passing of $5 \mathrm{~min}$, whichever occurred first. Data from all Day trials during which playback was started are presented here. Circles represent individual trials and lines are linear regression models. Significant $p$ values $(p<0.05)$ are in bold. Transformation for the $y$-axis units was $\log _{10}(s+1)$, where $s$ is time in seconds. In some trials, orient and pursuit occurred immediately upon prey reveal, thus time to these behaviors was assigned a value of $0 \mathrm{~s}$. 

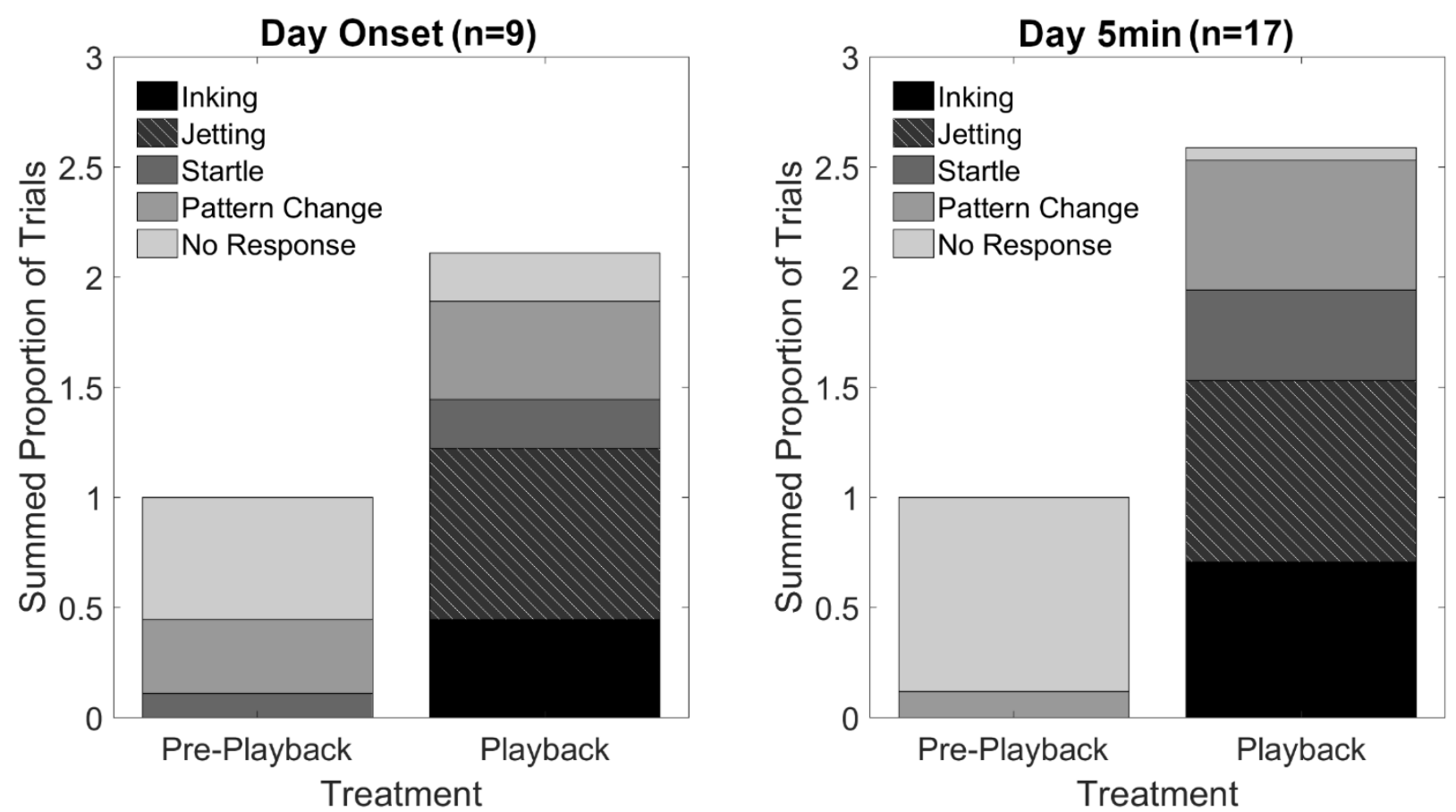

Figure 8. Summed proportion of trials in which squid exhibited a type of alarm response, or no response, during the last minute of the pre-playback period ("Pre-Playback") and during the first minute (first 30 pulses) of pile driving playback ("Playback"), for the Day Onset (left plot) and Day 5min (right plot) treatments. Only trials for which data were available for 30 pulses are shown here (playback for several Onset and Control trials was stopped before 30 pulses elapsed because the squid had captured the fish). Proportions were found individually for each behavior category, which, aside from 'No Response', are not mutually exclusive. Therefore, summed proportions may exceed 1. 


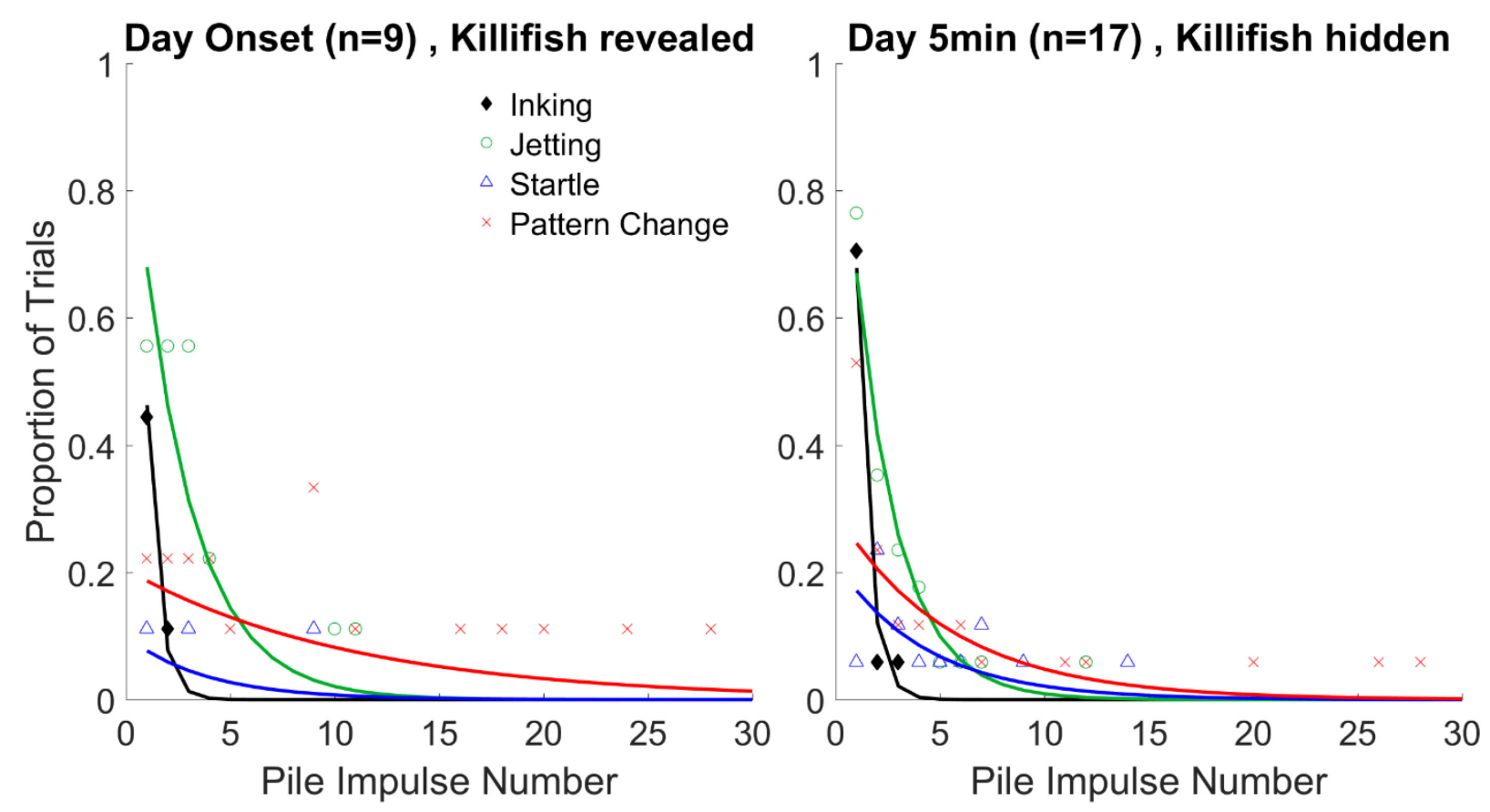

Figure 9. Proportion of trials squid exhibited each type of alarm response, at each pile impulse number, for Day Onset (prey already released) and Day $5 \mathrm{~min}$ (prey still hidden) trials. Each point in the scatterplot represents the proportion of trials in which a certain behavior occurred at that impulse number; solid lines of corresponding color are quasipoisson Generalized Linear Models (GLMs) for each response type. Only trials for which data were available for 30 pulses are shown here (playback for several Onset and Control trials was stopped before 30 pulses elapsed because the squid had captured the fish). 


\section{CHAPTER 4 : CONTEXT IS KEY: SQUID REPRODUCTIVE BEHAVIORS AND SPAWNING WITHSTAND WIND FARM PILE DRIVING NOISE}

This chapter has been submitted to Proceedings of the Royal Society B as: Jones, I. T., Schumm, M., Stanley, J. A., Hanlon, R. T., Mooney, T. A. (submitted July 7, 2021). Context is key: squid reproductive behaviors and spawning withstand wind farm pile driving noise.

The Supplementary Materials for this chapter can be found in Appendix C. 


\begin{abstract}
Anthropogenic noise is a pollutant inundating habitats worldwide, often adversely altering animal behavior and interactions. Yet, few studies have examined noise impacts on reproduction, nor empirically addressed how effects vary based on behavioral context. We investigated whether pile driving noise from offshore windfarm construction altered reproductive dynamics in mixed-sex groups of commercially and ecologically key squid, Doryteuthis pealeii. Pile driving construction noise is an intense, repetitive, far-reaching sound proliferating as the offshore wind energy industry expands globally and there is concomitant concern for its impacts on keystone species and vital fisheries taxa. Pile driving noise had no significant effects on occurrence rates of agonistic behaviors, mate guarding, mating, and egg laying, compared with silent control trials. These data suggest that the reproductive dynamics of squid may often be resilient to this increasingly pervasive environmental stressor. Results contrast starkly with behavioral response rates of the same squid species during feeding and resting and underscore that behavioral context guides how invertebrate taxa respond to noise. While some non-reproductive behaviors can clearly be disturbed, given that this is a short-lived semelparous species, results show that species with limited opportunity to reproduce can tolerate intense stressors to secure reproductive success.
\end{abstract}




\section{INTRODUCTION}

Humans are increasingly utilizing and developing coastal environments, with many activities having potential to impact marine taxa. The offshore wind energy industry is expanding globally, inducing concern over how anthropogenic noise pollution associated with construction, operation, and maintenance of wind farms will adversely impact aquatic wildlife (Mooney, Andersson, et al., 2020). Support vessels, sonars used for seismic surveys, construction equipment, and operational turbines emit anthropogenic noise during offshore wind farm development (Mooney, Andersson, et al., 2020). Impact pile driving during construction is considered the most intense and pervasive (Amaral et al., 2020). Pile driving involves repeated hammering of cylindrical piles into the seabed to support foundations for wind turbines, generally for those in water shallower than $60 \mathrm{~m}$ (Musial et al., 2019). It is a widespread noise source as pile driving is used in not just for the installation of windfarm turbine pilings, but also within harbors and coastal areas for a myriad of construction activities, in sum making it a sound of primary concern. To minimize negative impacts to marine life, studies investigating effects of pile driving noise on animal behaviors are crucial, especially behaviors that have direct implications for survival and reproduction.

Many studies have demonstrated effects of pile driving and other noise sources on the behavior of marine mammals and fish (Bruintjes et al., 2016; Graham et al., 2019; Nedelec et al., 2017), but far fewer have studied effects on marine invertebrates (Morley et al., 2014). Among fish, impulsive noise alters group cohesion and swimming dynamics of European seabass shoals (Dicentrarchus labrax); (Herbert-Read et al., 2017b; Neo et al., 2015, 2016). Fish exposed to pile driving noise also suffer temporary injuries to hearing structures and organs in close proximity to swim bladders (Casper, Smith, et al., 2013; Casper, Halvorsen, et al., 2013). For invertebrates (a diverse group of taxa), substrate vibrations from simulated pile driving cause filter-feeding mussels (Mytilus edulis) to reduce valve gape, (Roberts et al., 2015), elicit startle behaviors in hermit crabs (Pagurus bernhardus) (Roberts et al., 2016) and impair hermit crabs' (Pagurus acadianus) abilities to find key resources, i.e. empty shells (Roberts \& Laidre, 2019). 
There are no comparable data regarding impacts of far-reaching water-borne pile driving sound on invertebrates. The limited knowledge regarding impacts on numerous ecologically vital invertebrate taxa is both a concern and a risk as offshore windfarm construction progresses (Gedamke et al., 2016; Mooney, Andersson, et al., 2020). Furthermore, while ecological and behavioral contexts have been argued as critical to the management of anthropogenic noise pollution faced by marine mammals, (Ellison et al., 2012; Harris et al., 2018), such context-based approaches have not been addressed for most other marine taxa, including invertebrates.

Given this paucity of data, cephalopods (including squid, cuttlefish, and octopuses) are particularly important to examine anthropogenic noise impacts on, in part because of their high ecological and commercial relevance. They made up $4.5 \%$ of global capture production (tonnes) and 6.2\% of global fishery export values (USD) from 2012 to 2018 (FAO, 2021), and are key central trophic links in food webs (Boyle \& Rodhouse, 2005). Squid for example can constitute over $50 \%$ of regional seabird diets and up to $95 \%$ of odontocete diets [ibid]. Longfin squid (Doryteuthis pealeii) are of particular concern with respect to potential impacts of wind farm construction. This species is commercially fished in the United States, having average annual landings of about 11,000 mt and annual values of $\$ 30$ million since 2010 (NMFS, 2021). Lease areas for offshore windfarms in the northeast U.S. overlap or are adjacent to inshore spawning and fishing areas for D. pealeii (MARCO, 2021), and construction is planned within these areas from 2021 to beyond 2025 (BOEM, 2021; Musial et al., 2020). These squid detect and behaviorally respond to sounds at frequencies below $1000 \mathrm{~Hz}$ (Mooney et al., 2010; Mooney, Samson, et al., 2016), suggesting sound-sensitivity overlapping with the typical peak frequencies of pile driving noise. Ecological functions of cephalopod sound detection are unknown; however, they are thought to utilize sound and vibration for predator avoidance and possibly for developing an "auditory scene" to orient to and navigate in their environment, as has been discussed for fishes (Fay, 2009; York \& Bartol, 2014).

Moreover, key studies of their behavior, reproduction and sound-sensitivity provide a vital foundation, enabling this taxon to act as a model to address invertebrate noise impacts. Several studies have reported disruptive behavioral and physical effects of anthropogenic noise 
on cephalopods. Squid display alarm responses normally utilized for predator evasion, such as inking and jetting, during air gun (Sepioteuthis australis) and pile driving noise (D. pealeii) (Fewtrell \& McCauley, 2012; Jones et al., 2020). Pile driving noise also causes D. pealeii to cease their pursuit of prey (Jones et al., 2021). Additionally, noise may cause physical damage to cephalopod sound detection structures (statocysts) (Solé et al., 2013, 2017). To the authors' knowledge, to date no studies have addressed impacts of anthropogenic noise on reproductive behaviors of cephalopods, nor any marine invertebrate.

Like many cephalopods, $D$. pealeii is short-lived, and has an average lifespan of less than a year (Brodziak \& Macy, 1996; Macy \& Brodziak, 2001), mating for a short time period from May to October. Although they can mate and lay eggs multiple times over several weeks (Hanlon et al., 2013; Maxwell et al., 1998) they are considered semelparous because they only breed for one season and then senesce, making mating events highly important to individual fitness (Shashar \& Hanlon, 2013). Complex behavioral dynamics are associated with inshore mating and spawning of $D$. pealeii around communal egg beds and spawning dynamics are welldescribed (Shashar \& Hanlon, 2013). Briefly, females lay gelatinous capsules containing eggs on the substrate, which form large bundles called "egg mops". Males are visually attracted to and approach egg mops. The capsules contain a contact pheromone that elicits heightened aggression in males, leading to subsequent agonistic (fighting) behaviors (Buresch et al., 2003, 2004; Cummins et al., 2011). Males take on flexible mating roles according to their size relative to nearby males, and their success or failure in agonistic bouts. "Consort males" (usually larger males) will pair with and swim parallel to a female, and "guard" her from other males. Consort males perform the majority of successful mating. "Sneaker males" are usually smaller and keep their distance from larger males, but may quickly jet toward a female, attempting to mate with her. With either consort or sneaker males, mating involves transfer of sperm to the female, which the female then uses to fertilize egg capsules as she extrudes them.

The present study investigated anthropogenic noise impacts on reproductive behaviors of small mixed-sex groups of longfin squid Doryteuthis pealeii, via controlled laboratory experiments using underwater playbacks of pile driving sound recorded during wind farm construction. To determine if this noise impacted reproduction, we measured a suite of 
reproductive behaviors including: mate guarding (when consort males actively position themselves between a female and other males and defend that female resource), as well as male-male agonistic (fighting) behaviors, mating, and egg laying. To evaluate contextdependent effects, we compared response rates during reproductive activities to those of parallel studies with similar methods addressing noise-induced disturbances during feeding and resting.

\section{METHODS}

\section{Animal husbandry}

Squid were collected by trawl in Vineyard Sound, MA, USA from May-July 2019 by the Marine Biological Laboratory (Woods Hole, MA). Squid were transported to the nearby Environmental Systems Lab (ESL) at Woods Hole Oceanographic Institution and housed in flowing-seawater tanks prior to testing. Males and females were kept in separate tanks and male squid were further separated by relative size ("large" and "small"). Water temperature in holding tanks was maintained at $18-20^{\circ} \mathrm{C}$ and squid were exposed to a natural light cycle. Squid were fed killifish (Fundulus spp.) ad libitum daily and kept in holding tanks for 1-5 days before experimentation. Only visibly healthy squid with minimal skin damage were tested.

\section{Experimental setup}

Experiments occurred in a cylindrical, 1.8-m-diameter tank in the ESL during daylight hours. Water was maintained at a depth of $0.82 \mathrm{~m}$ and temperature of $18.0 \pm 1.2^{\circ} \mathrm{C}$ (mean \pm SD). A slow water inflow maintained tank temperature and high dissolved oxygen levels. The bottom of the tank had a $3 \mathrm{~cm}$ layer of sand. An Aqua-30 speaker (DNH, Netherlands; frequency response: $0.08-20 \mathrm{kHz}$ ) and hydrophone (High Tech Inc., USA; sensitivity: $-165 \mathrm{~dB}$ re $1 \mathrm{~V} / \mu \mathrm{Pa}$; frequency response: $0.002-30 \mathrm{kHz}$ ) were inserted into the tank to project and monitor sound respectively during experiments. The hydrophone was connected to a SoundTrap ST4300 acoustic recorder (Ocean Instruments, NZ; 4 dB gain) located outside the tank. Trials were recorded with an overhead camera (Sony Handycam HDR-XR550), and two underwater cameras 
(GoPro Hero 4) facing each other from opposing sides of the tank at mid-depth, so that video of the entire inside of the tank was captured.

\section{Mate guarding experiment}

During experiments, squid were exposed to either 5-min long pile driving playbacks or 5min long silent control playbacks (hereafter referred to as "pile" and "control" trials, respectively). Originally, pile driving files had an inter-pulse interval of $1.8 \mathrm{~s}$, and were recorded from a hydrophone array $500 \mathrm{~m}$ away from a pile driven for the Block Island Wind Farm, $1 \mathrm{~m}$ above the seabed, in water $26 \mathrm{~m}$ deep (Amaral et al., 2018, 2020). These field data, along with particle acceleration calculated from hydrophones in the field, were provided to the authors (see Acknowledgements). Three distinct pile driving playback files were prepared using Adobe Audition (version 3.0), with pulses of equal amplitude arranged in randomized order for a $1 \mathrm{~min}$ loop, which was looped five times for a total 5 min duration. All files were edited to have a consistent inter-pulse interval of $2 \mathrm{~s}$.

Squid were tested in groups of three, consisting of a large male, a small male, and a female, with respective mean $\pm S D$ mantle lengths of $22.5 \pm 3.3 \mathrm{~cm}, 15.6 \pm 2.2 \mathrm{~cm}$, and $14.3 \pm$ $1.5 \mathrm{~cm}$. Mantle lengths of each large male and small male pair differed by at least $3.5 \mathrm{~cm}$ (mean \pm SD: $6.9 \pm 2.1$ ). Large males were expected to act as consort males, and small males were expected to act as sneakers (Shashar \& Hanlon, 2013). All cameras were in place and recording before squid were added to the experiment tank. Squid were added sequentially to the tank at intervals of two minutes in a consistent order of large male, small male, then female. Immediately following the addition of the female to the tank, the SoundTrap was turned on to start monitoring sound levels in the tank. Two minutes after the female was added, an egg mop was added to the center of the tank, and a second egg mop was added near the tank wall (approximately a quarter of the tank's circumference away from the speaker). The addition of the egg mops marked the start time of each trial. Egg mops induced male aggression and mate guarding (Buresch et al., 2003; Cummins et al., 2011).

For all trials, squid were given at least ten minutes to habituate to the experiment tank before starting playback. An experimenter was stationed out of view of the squid and watched 
the trial on a screen to monitor the occurrence of mate guarding. Criteria for mate guarding were that the male remained within two body lengths of the female and actively positioned itself between the female and other male (Shashar \& Hanlon, 2013). Playback was started when mate guarding was observed for 15 s continuously (after the $10 \mathrm{~min}$ habituation period had passed). Although this meant the duration between the start of the trial and start of the first playback varied (median [IQR]: 14.3 [11.1-21.3] min), this playback start criterion was chosen because the experimental design was primarily focused on testing whether noise disrupted mate guarding. Playbacks were repeated up to three times if squid resumed mate guarding after the first and second exposure, with a minimum of 10 min of quiet (i.e. no playback) in between. Agonistic behaviors, including number of chases (forward acceleration in pursuit of another squid) and lunges (forward acceleration followed by attempt to grab another squid) by males were also quantified, as defined previously by Cummins et al. (2011).

Fifty-five trials were conducted. Of these, 30 trials had males that mate guarded (15 pile trials, 15 control trials) and were used for analyses. One control trial and one pile trial had a single playback period; the remaining 28 trials had three playback periods. Two trained observers watched videos and recorded time spent mate guarding, number of chases, and number of lunges during 5-min-long "playback" periods, and 5-min-long periods just preceding the second and third playback periods ("quiet1" and "quiet2", respectively). One observer analyzed half of the trials ( $n=15)$, and both observers analyzed the other half. For the trials analyzed by both observers, inter-observer reliability was high for both mate guarding and agonistic behaviors ( $r>0.96$, Pearson correlation). Mating and egg-laying events were also scored by one observer who watched the entire $(1-2 h)$ duration of each trial.

\section{Acoustic calibration of the experimental tank}

Mapping of the sound field in the experimental tank involved similar methods and instrumentation used in prior studies (Jones et al., 2021, 2020). Briefly, 1 min of a pile driving file (same as used in experiments) was played through the speaker and recorded in $20 \mathrm{~cm}$ increments in all three dimensions (240 recording positions total). Cephalopods detect acoustic particle acceleration rather than pressure (Budelmann, 1992b; Budelmann \& Tu, 1997; Mooney 
et al., 2010), therefore, particle acceleration was recorded, as well as sound pressure for comparison with other studies. Recording instruments were affixed at the end of a PVC probe in the following configuration: a PCB triaxial accelerometer (model W356B11, frequency response: $0.5 \mathrm{~Hz}-5 \mathrm{kHz}$, sensitivity of each axis: $1.04 \mathrm{mV} / \mathrm{m} \mathrm{s}^{-2}$ ) was centered at the recording position, and a Reson hydrophone (model TC4013, frequency response: $1 \mathrm{~Hz}-170 \mathrm{kHz}$, sensitivity: $-211 \mathrm{~dB}$ re $1 \mathrm{~V} / \mu \mathrm{Pa}$ ) was spaced $1.5 \mathrm{~cm}$ to the left of the accelerometer (facing the speaker).

Acoustic data analyses were performed following previous methods (Jones et al., 2020), and are briefly summarized here. Zero-to-peak levels of individual pile pulses, in decibels (dB), were calculated for particle acceleration $\left(S A L_{z-p k}\right)$ and sound pressure $\left(S P L_{z-p k}\right)$ as follows:

$$
S A L_{z-p k} \text { or } S P L_{z-p k}=20 * \log _{10}\left(X_{p k}\right)
$$

where $X_{p k}$ is the maximum absolute acceleration $\left(\mu \mathrm{m} \mathrm{s}^{-2}\right)$ or pressure $(\mu \mathrm{Pa})$. For simplicity, the 3D vector (Euclidean) norm of particle acceleration was calculated, and its magnitude is reported. Power spectral density (PSD) was calculated to visualize these magnitudes across frequencies. All acoustic metrics were limited to 20-1000 Hz, encompassing the hearing range of $D$. pealeii. Acoustic analysis results can be found in the electronic supplementary material.

\section{Statistical methods}

Statistics were performed in R version 4.0.4 using the Ime4 (Bates et al., 2015), car (Fox \& Weisberg, 2019), and glmmTMB (Brooks et al., 2017) packages. The significance threshold for all tests was $\alpha=0.05$. Generalized linear mixed effects models (GLMMs) were used to fit mate guarding and agonistic data with repeated measures. Proportion of time spent mate guarding was fit with a beta distribution, and counts of chases and lunges were fit with a negative binomial distribution. Treatment (pile vs. control) and period ("playback" periods 1, 2 and 3, and "quiet" periods 1 and 2) were included as main effects and squid individual was a random effect. Interactions between treatment and period were kept in final models if they were significant. Model best fit was selected according to Akaike information criteria (AIC). Post-hoc Wald type II $\chi^{2}$ tests were used to test significance of fixed factors. 


\section{RESULTS}

\section{Mate guarding}

Large males mate guarded the female squid in 25 of the 30 trials (12 control, 13 pile), and the small male mate guarded in eight trials ( 5 control, 3 pile). In three trials ( 2 control, 1 pile), the small male mate guarded first and the large male later took over as consort and mate guarded for the rest of the trial. These consort role shifts took place early in the trials, either before playback1, during quiet1, or during quiet2 periods. The number of mate-guarding small males was too low to perform GLMMs including all period and treatment groups, thus statistics were limited to large males.

There was no significant effect of the pile driving sound on time spent mate guarding by large males. Inter-quartile ranges of time spent mate guarding in pile and control treatments largely overlapped, whether comparing these treatments within playback periods, within quiet periods, or comparing playback and quiet periods within a given sound treatment (Fig. 1a). This reflects the similarity of behaviors in noise vs. quiet conditions, despite the high amplitude pile driving sound (Fig. S1). Analyzing all large males (Fig. 1a), period was a significant factor $\left(\chi^{2}=\right.$ $10.01, d f=4, p=0.04)$, treatment was not significant $\left(\chi^{2}=0.88, d f=1, p=0.35\right)$, and the interaction between period and treatment was significant $\left(\chi^{2}=14.0, d f=4, p<0.01\right.$; Table S1). For small males, mate guarding between pile and control treatments was more variable (Fig. 1b); differences between pile and control proportions (within a period) were likely due to individual effects of different small males rather than noise effects, since proportions within either sound treatment were more similar between playback and quiet periods. We analyzed large males in trials where small males did not mate guard to determine whether the significant period effect may have been due to consort role shifts from the small male to large male. In this subset of trials ( $n=10$ control, $n=12$ pile) period was not a significant factor $\left(\chi^{2}=2.69, d f=4, p=\right.$ $0.61)$, nor was treatment $\left(\chi^{2}=0.01, d f=1, p=0.93\right.$; Table S2). This suggests that the delay of several large males $(n=3)$ in taking on a consort role led to the significant time period factor for data in Fig. 1a. 


\section{Agonistic behaviors}

Pile driving noise did not have a significant effect on agonistic behaviors of large males, in terms of number of chases and number of lunges toward competing males (Fig. 2). For number of chases, neither period $\left(\chi^{2}=1.43, \mathrm{df}=4, \mathrm{p}=0.84\right)$ nor treatment $\left(\chi^{2}=1.88, \mathrm{df}=1, \mathrm{p}=\right.$ 0.17) were significant factors (Table S3). Similarly, for number of lunges, period $\left(\chi^{2}=1.05, d f=\right.$ $4, p=0.90)$ and treatment $\left(\chi^{2}=0.19, d f=1, p=0.67\right)$ were not significant (Table S4). There were no significant interactions between periods and treatment for chase or lunge behaviors. Small males did not chase or lunge at large males. Overall, these results indicated that aggressive behaviors of male squid were unaffected by the noise treatment.

\section{Mating and egg-laying}

Mating and egg-laying continued to occur during and after noise exposure. Mating occurred in $30 \%$ of trials ( 5 control, 4 pile), and was always between the large male and female. Mating between a given consort pair occurred once in six trials, twice in two trials, and thrice in one trial. There was no significant difference in duration of mating events between pile and control trials $(U=18, p=0.10$, Mann-Whitney $U$ test). Overall the median duration of the typical male parallel mating position was $17 \mathrm{~s}$ (IQR: 15-18), and durations ranged from 9-33 s, within those observed in wild squid (Shashar \& Hanlon, 2013). In two pile trials, noise playback (playback1 or playback3) started during mating, and mating continued. Durations of these two mating events were 18 and $28 \mathrm{~s}$, within the duration range of mating events that occurred in "quiet" periods and control playbacks. Egg laying (by females) also occurred after mating at statistically similar rates in pile and control trials (mean \pm SD: $0.90 \pm 0.30$, and $0.74 \pm 0.14$ eggs capsules $\min ^{-1}$, respectively; $U=21, p=0.41$, Mann-Whitney $U$ test).

\section{DISCUSSION}

\section{Resilience of reproductive behaviors during noise}

Here we present the first empirical dataset addressing potential effects of anthropogenic noise on reproductive behaviors of a marine invertebrate. Overall, there was no 
indication that high-intensity, repeated pile driving noise impacted any of the suite of reproductive behaviors measured, including agonistic, mate guarding, mating, or egg-laying behaviors. Typical behavioral dynamics (Shashar \& Hanlon, 2013) of sexually active squid continued to occur despite the repeated, high-intensity, impulsive noise treatment. These results are perhaps surprising given the array of impacts seen in other behaviors of cephalopods and in other taxa (Mooney, Andersson, et al., 2020); however, they underscore the exceptionally strong motivation of these squid to reproduce. Squid engaging in these behaviors are nearing the end of their lifespan; females may continue to mate with multiple males and spawn over a few weeks, but both sexes will soon senesce (Hanlon et al., 2013; Maxwell \& Hanlon, 2000). From an evolutionary standpoint, persistence of reproductive behaviors during environmental stressors is advantageous for species with limited opportunity to reproduce in their lifetime. The present results are consistent with theory that reproductive behaviors of semelparous species should be relatively uninfluenced by potentially inhibitory effects of stress (de Jong et al., 2020; Wingfield \& Sapolsky, 2003). Based on these data, one might conclude that mating behaviors of semelparous species are at a lower risk of adverse effects from noise exposure.

Of course it is not possible to generalize these trends to all semelparous species, all cephalopods, or all noise types, since responses may be specific to species and noise characteristics. For instance, females of one semelparous goby species (Pomatoschistus microps) significantly delayed their inspection of nests, delayed spawning, and laid fewer eggs during noise from airstones (Blom et al., 2019). These differences only occurred when gobies were presented with continuous, rather than impulsive, noise. It is possible that squid behaviors in the present study may have differed if presented with a different noise type (e.g., boat noise). Continuous noise with irregular amplitude and frequency characteristics is thought to be more likely to cause stress compared to impulsive sounds that have more consistent amplitude and frequency spectra (de Jong et al., 2020). Impulses played in the present study were similar to each other in amplitude and spectra and may be considered "regular," although amplitudes received by squid varied spatially in the tank. Effects of continuous or irregular noise on squid behavior remain to be tested. 
Laboratory-based studies allow detailed observation of behavior in a well-controlled environment without confounding influence of extraneous noise sources and other environmental factors. Peak particle acceleration levels in the experiment tank (see electronic supplementary material) exceeded those measured at $500 \mathrm{~m}$ from Block Island Wind Farm piles ( $1 \mathrm{~m}$ above seabed, $26 \mathrm{~m}$ depth), thus representing acceleration levels predicted within a $500 \mathrm{~m}$ radius (Amaral et al., 2018). Importantly, sound propagation from piles depends on multiple engineering and environmental factors, including pile dimensions, angle with respect to the seabed, hammer strike energy, bathymetry, sediment properties, and seasonally-dependent sound speed profiles; however, propagation of particle acceleration from piles is poorly understood (Lin et al., 2019; T. Lippert \& von Estorff, 2014; Tsouvalas \& Metrikine, 2016). Intank underwater acoustics cannot exactly replicate in-situ acoustic propagation (Jézéquel et al., 2019; Jones et al., 2019; Parvulescu, 1964; Rogers et al., 2016). Yet the experimental acoustic field can be measured precisely in high spatial resolution (perhaps more so than in the field), and careful effort was made to represent similar amplitudes and frequency spectra that squid may experience from in-situ pile driving.

\section{Context-dependent noise impacts on squid: cross-study comparisons}

Comparisons to response rates of squid in other behavioral conditions (but similar methodological setups) underscores that context of noise exposure greatly affects responses exhibited (Fig. 3). Previous studies of individual squid either simply swimming, resting or otherwise not engaged in specific tasks (Jones et al., 2020), or during feeding events, showed substantially higher rates of alarm and flight responses (Jones et al., 2021). Alarm responses included inking, jetting, other locomotor startle behaviors, and body pattern changes, which are all employed by squid as anti-predator defenses. Comparatively, noise effects on feeding behaviors were more nuanced. There were no statistically significant differences between noise and control treatments in the proportion of squid that ultimately captured prey during the trial. Yet noise played during squids' pursuit of prey led to a significant increase in missed or abandoned prey capture attempts. 
In the present study, trios of squid exposed to high intensity noise did not demonstrate any significant changes in reproductive behaviors. Collectively, these studies emphasize the importance of behavioral context when predicting anthropogenic noise effects on marine taxa. This theme has been demonstrated across vertebrate and invertebrate taxa (Bruintjes \& Radford, 2013; Ellison et al., 2012; Filiciotto et al., 2018). For example, boat noise caused cichlids (Neolamprologus pulcher) to change rates of digging and attack behaviors when eggs were not present, but there were no significant changes to these behaviors when eggs were present (Bruintjes \& Radford, 2013). In the case of longfin squid, evidence from laboratory experiments indicate noise exposure is potentially more disruptive to anti-predator responses and feeding behaviors than to reproductive activities.

\section{Conclusions and future directions:}

The present study indicates that reproductive behaviors of longfin squid may be less at risk of being disrupted by noise relative to anti-predator defense and feeding behaviors. Together with previous studies on $D$. pealeii, these results reinforce the importance of considering multiple ecological contexts in which animals' responses to anthropogenic noise may differ. To address noise effects in more ecologically and acoustically relevant scenarios, complementary field studies on squid behavioral responses to noise are needed. Laboratory studies have provided valuable information on the contexts in which squid behavior may be adversely affected by anthropogenic noise and can inform research foci for field studies addressing in-situ and population-level impacts. These research efforts and the results of the present study are of central importance to the fishing industry, regulators, and energy industry seeking to assess and address risks that offshore energy expansion poses to ecologically and commercially key marine fauna. 
a)

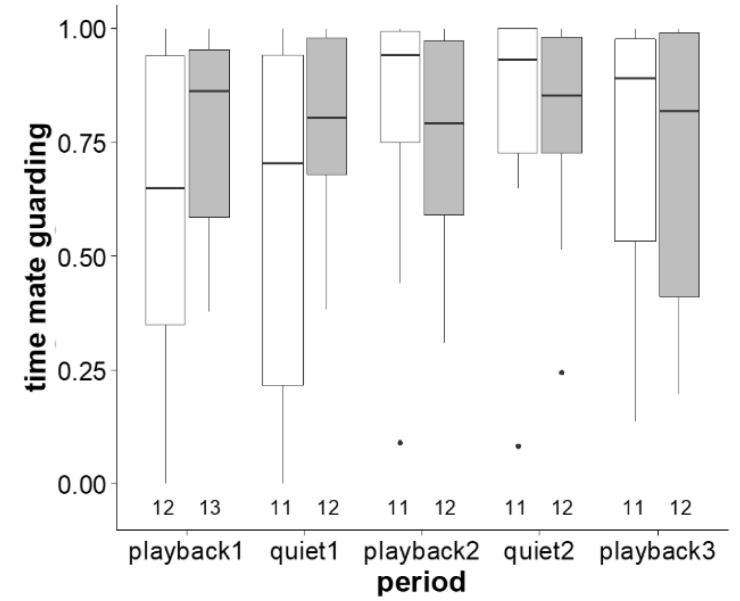

b)

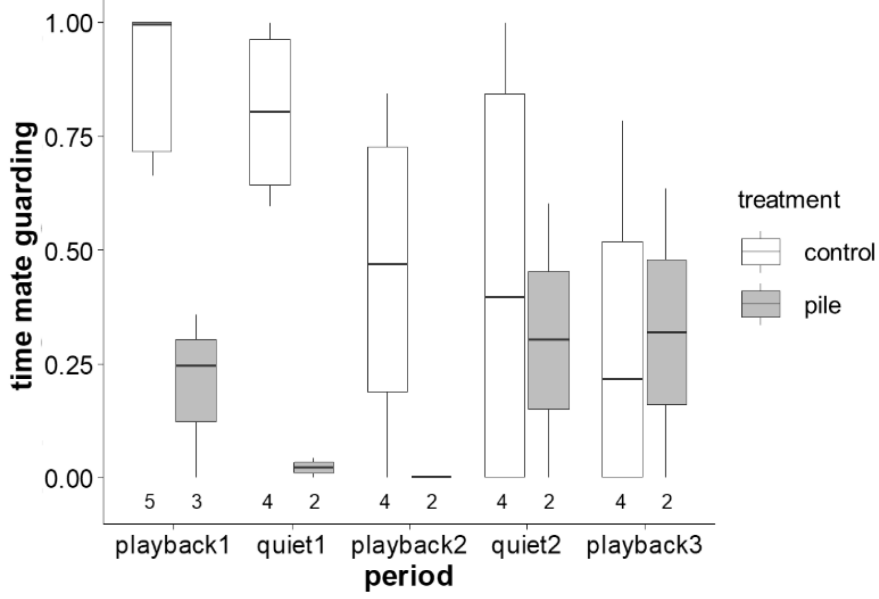

Figure 1. Proportion of time spent mate guarding in playback and between-playback (quiet) time periods for a) large males, and b) small males. Periods each had 5 min duration and are listed in the sequence they were presented to the squid. Numbers under each box are sample sizes. Horizontal lines represent medians, boxes extend from the $25^{\text {th }}$ to $75^{\text {th }}$ percentile, and dots represent outliers, defined as values smaller than 1.5 times the inter-quartile range. 
a)

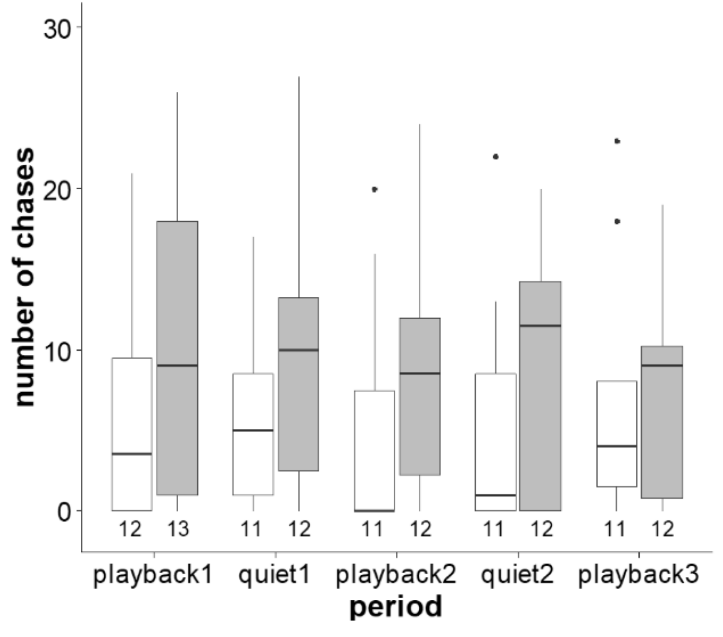

b)

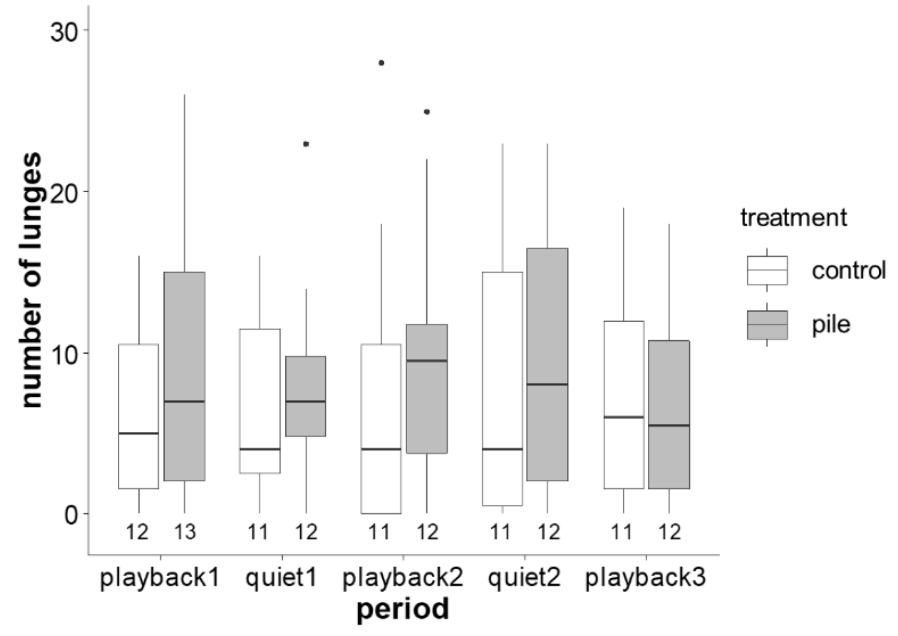

Figure 2. a) Number of chases, and b) number of lunges toward small males by the same large males shown in Fig. 1a, in playback and between-playback (quiet) time periods. Periods each had 5 min duration and are listed in the sequence they were presented to the squid. Numbers under each box are sample sizes. Horizontal lines represent medians, boxes extend from the $25^{\text {th }}$ to $75^{\text {th }}$ percentile, and dots represent outliers, defined as values greater than 1.5 times the inter-quartile range. 


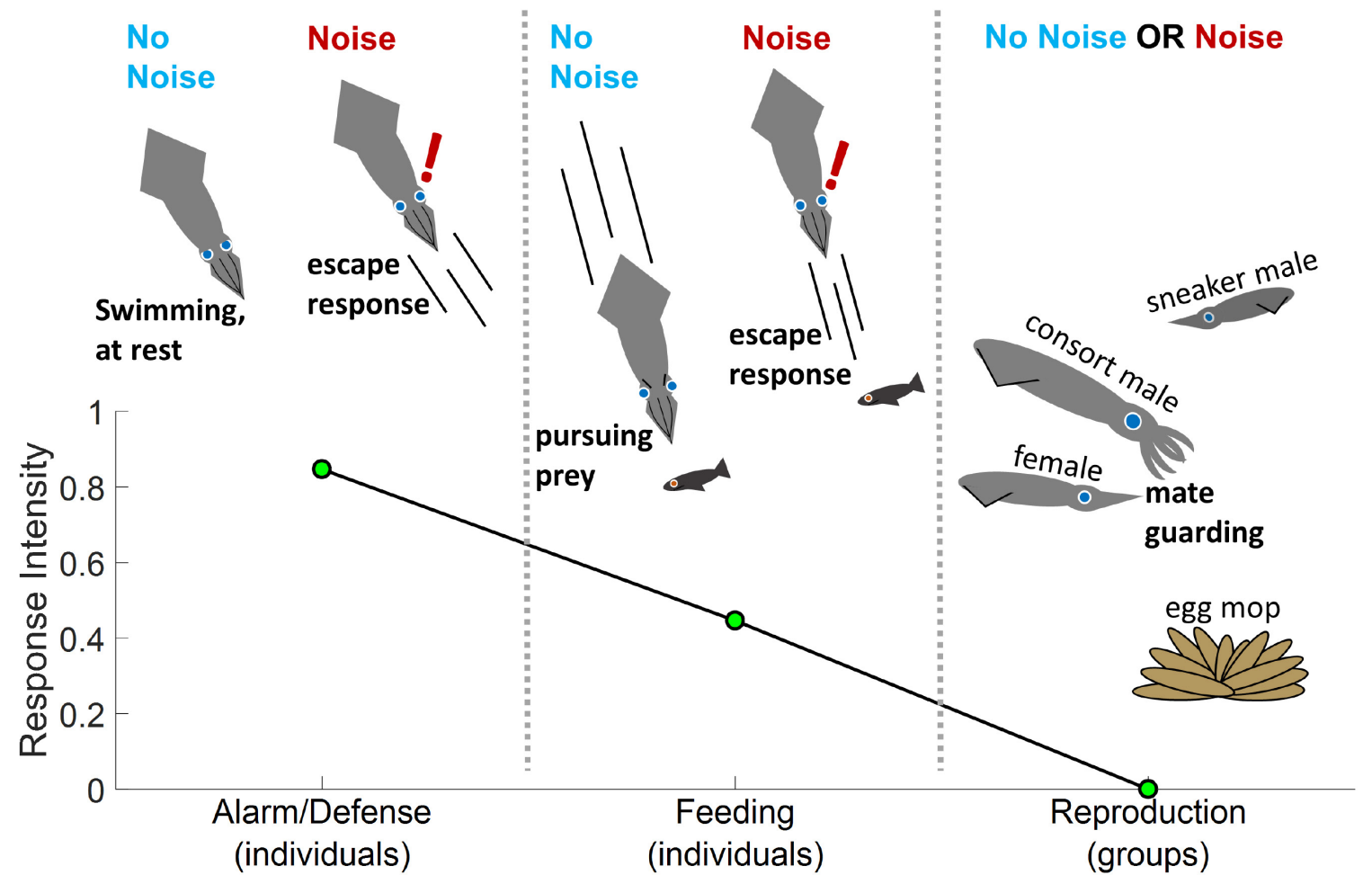

Figure 3. Comparison of relative response rates of $D$. pealeii to pile driving noise across studies measuring alarm/defense behaviors (Jones et al., 2020), feeding behaviors (Jones et al., 2021), and reproductive behaviors (present study). Response intensity is the quantified proportion of noise exposure trials with a response subtracted by the proportion of control trials with a response. For the alarm/defense study, a response was defined as at least one occurrence of inking, jetting, startle, or body pattern change. For the feeding study, a response was defined as at least one missed predation attempt. Since there were no significant noise effects on any reproductive behaviors, response intensity for the present study is set at zero. Example videos showing these behaviors during noise are in the electronic supplementary material. 
CHAPTER 5 : NATURAL CUES FOR INVERTEBRATE AND FISH HEARING: PARTICLE MOTION MEASUREMENTS ON REEFS 


\begin{abstract}
Coral reef soundscapes are increasingly studied for their ecological uses by invertebrates and fishes, their applications for monitoring habitat quality, and to investigate effects of anthropogenic noise pollution. Few examinations of aquatic soundscapes have reported particle motion levels and variability, despite their relevance to invertebrates and fishes. We quantified ambient particle acceleration from orthogonal hydrophone arrays over several months at four coral reef sites, which varied in benthic habitat and fish communities. Particle acceleration magnitudes were similar across axes, within $3 \mathrm{~dB}$. Temporal trends of particle acceleration corresponded with those of sound pressure, and the strength of diel trends in both metrics significantly correlated with percent coral cover. Higher magnitude particle accelerations diverged further from pressure values, potentially representing sounds recorded in the near field. Particle acceleration levels were also reported for boat and example fish sounds. Comparisons with particle acceleration derived audiograms suggest greatest capacity of invertebrates and fishes to detect soundscape components below $100 \mathrm{~Hz}$, and poorer detectability of soundscapes by invertebrates compared to fishes. Based on our results, we discuss research foci for which reporting of particle motion is essential, versus those for which sound pressure may suffice.
\end{abstract}




\section{INTRODUCTION}

Coral reefs are biodiverse habitats and have complex soundscapes consisting of biological, geophysical, and anthropogenic sounds. Passive acoustic monitoring of these soundscapes is essential for understanding acoustic ecology of coral reefs, and can be applied to assess and monitor local biodiversity (Mooney, lorio, et al., 2020). As a growing number of studies describe marine soundscapes, the ecological importance of natural soundscape cues to invertebrates and fishes has been increasingly realized (Popper \& Hawkins, 2018; Putland et al., 2019). Reef soundscapes may aid pelagic larvae and juveniles of coral reef taxa navigating toward reefs (Radford, Stanley, et al., 2011). Numerous studies have demonstrated phonotaxis (movement toward sound) and increased settlement when exposed to reef sounds, of corals (Lillis, Apprill, et al., 2018; Lillis et al., 2016), oysters (Lillis et al., 2013), crabs (Stanley et al., 2010, 2011, 2012), and reef fishes (Gordon et al., 2018; Parmentier et al., 2015; Suca et al., 2020). To sound-sensitive taxa, biological soundscape cues provide critical ecological information about the presence and intent of predators, competitors, prey, and potential mates (Farina \& Gage, 2017). Specific cues within soundscapes may be utilized for communication in competitive or reproductive contexts, as demonstrated in many reef fishes (Lobel et al., 2010; Tricas \& Boyle, 2014) and in crustaceans (Buscaino et al., 2015; Jézéquel et al., 2020; Lillis et al., 2017; Popper et al., 2001). More basally, animals may listen to soundscape cues to orient themselves, navigate, and locate sound-producing organisms in their habitat (Fay, 2009).

Underwater soundscapes have almost exclusively been quantified and described in sound pressure, despite a growing appreciation of particle motion's relevance to invertebrate and fish hearing. A handful of studies have reported particle motion of soundscapes, typically quantified as particle acceleration ( $\mathrm{dB}$ re $1 \mu \mathrm{m} \mathrm{s}^{-2}$ ) which is considered the relevant transduction stimulus for sound-detection organs of invertebrates and fishes (Popper \& Hawkins, 2018). These studies examined ambient particle motion in diverse habitats, from coral reefs in the Pacific (Horch \& Salmon, 1973; Kaplan \& Mooney, 2016), to a sandy tropical bay in Brazil (Jesus et al., 2020), to freshwater rivers and streams (Lugli \& Fine, 2007; Lumsdon et al., 
2018). Others have addressed particle motion of vessel noise (Magnhagen et al., 2017; McCormick et al., 2018; Nedelec et al., 2014; Picciulin et al., 2010) and wind turbine noise (Sigray \& Andersson, 2011; Wahlberg \& Westerberg, 2005). These evaluations reported particle motion only for short time periods (as long as two continuous days), thus temporal variability (a key parameter, at least for pressure) of particle motion soundscapes remains poorly understood (Mooney, lorio, et al., 2020). Further, particle motion of specific soundscape components such as fish calls is largely undescribed.

Fishes and invertebrates primarily detect particle motion at frequencies below $1000 \mathrm{~Hz}$, and many detect infrasound, i.e., below $20 \mathrm{~Hz}$ (Packard et al., 1990; Sand et al., 2000; Wilson et al., 2018). Predominant coral reef sounds within their hearing ranges include abiotic sounds from wind and wave motion, often below $100 \mathrm{~Hz}$, and sounds produced by fish, often below $1000 \mathrm{~Hz}$ (Montgomery et al., 2006; Tricas \& Boyle, 2014). Anthropogenic boat noise is also in this frequency range, and is present daily in many nearshore habitats, including coral reefs (Butler et al., 2021; Dinh et al., 2018). Broadband choruses of snapping shrimp snaps are omnipresent in many coral reef soundscapes, and usually have peak frequencies at or above $2000 \mathrm{~Hz}$ (Au \& Banks, 1998; Lillis \& Mooney, 2018). Therefore, the detectability of these snaps among invertebrates and fishes is likely limited compared to lower-frequency fish and abiotic sounds (Salas et al., 2018).

Measurement of particle motion is important when studying coral reef soundscapes for several reasons. First, it may not be assumed $a$ priori that particle motion levels always scale with sound pressure levels. Many coral reefs are relatively shallow and have rugose benthos; in such environments, empirical particle motion is more likely to deviate from that predicted by theory for a plane wave, especially for low frequencies, e.g., below $100 \mathrm{~Hz}$ (Gray et al., 2016; Nedelec et al., 2016). This necessitates either direct measurement of particle motion (e.g., with an accelerometer) or calculation from pressure differentials using a hydrophone array.

Second, by reporting magnitudes of particle motion, one can better estimate the detectability of soundscape cues for invertebrates or fishes. Particle motion may have different propagation losses and signal to noise ratios compared to those of pressure (Jesus et al., 2020; Kalmijn, 1988). Even for species with both particle acceleration and pressure sensitivity, shapes 
of hearing threshold curves may differ for each metric (Dale et al., 2015; Horodysky et al., 2008). Comparisons of particle motion soundscape measurements with animals' particle motion detection thresholds are needed to address questions regarding the detectability and ecological functionality of natural soundscape cues for these taxa.

Further, particle motion is inherently directional at a given point, whereas sound pressure is not. This directionality plays important (though often poorly understood) roles in how invertebrates and fishes process acoustic cues to identify, localize, and behaviorally respond to sounds in their environment (Wilson et al., 2018; Zeddies et al., 2012). Directional particle motion data may help us discern how reef animals use acoustic cues to enact fundamental processes such as navigation, selection of settlement sites, avoidance of predators, and communication.

Quantification of underwater soundscapes is actively being pursued as a low-cost and high temporal-resolution approach to monitor habitat health (Mooney, lorio, et al., 2020). Trends of sound pressure levels on coral reefs, particularly those below $2 \mathrm{kHz}$, are often positively correlated with visually-measured indicators of reef health such as coral cover and fish biomass (Freeman \& Freeman, 2016; Kaplan et al., 2015; Staaterman et al., 2017). Though temporal and spectral acoustic trends are site-specific (Radford et al., 2014), soundscape analyses show promise as effective means for long-term monitoring of biodiversity (Mooney, Iorio, et al., 2020). Yet, such associations between acoustic and non-acoustic indicators have not been addressed for particle motion.

The presence and influences of anthropogenic sounds on coral reefs are also important to understand. Noise from recreational vessels and commercial shipping is frequent in many coastal habitats, including coral reefs (Bittencourt et al., 2020; Dinh et al., 2018; Kaplan \& Mooney, 2015). Boat noise can have a multitude of adverse effects on invertebrates and fishes, such as physiological changes indicative of stress (Filiciotto et al., 2016), disruptions to feeding, antipredator, settlement, or orientation behaviors (Bruintjes et al., 2016; Holles et al., 2013; Magnhagen et al., 2017; Mensinger et al., 2018; Simpson et al., 2016; Wale et al., 2013a), and masking of ecologically relevant cues (Pine et al., 2016). Given that the hearing of invertebrates and most fishes is dependent on particle motion, and such signals are not easily predicted in 
the field, it seems vital that masking and other noise effects are quantified in sensory-relevant particle motion.

The present study is the first to quantify particle motion levels of coral reef soundscapes. This study was conducted at reefs on the southern shore of St. John, U.S. Virgin Islands. These reefs have been the focus of ecological research for decades (Edmunds, 2013; Edmunds et al., 2020). Recent studies focusing on sound pressure have investigated multiple facets of soundscape ecology at these sites, including temporal variability (Kaplan et al., 2015), across-reef spatial variability (Lillis, Caruso, et al., 2018), patterns in snapping shrimp activity (Lillis \& Mooney, 2018), settlement behavior of corals (Lillis, Apprill, et al., 2018), larval fish settlement (Suca et al., 2020), and boat noise patterns (Dinh et al., 2018; Kaplan \& Mooney, 2015). These studies provide valuable observations based on sound pressure, with which we can compare trends in particle motion data.

The primary goals of this study were to describe particle motion magnitudes of coral reef sounds, how they correlate with sound pressure, and the extent to which they may be detectable by marine invertebrates and fishes. We also aimed to describe how particle motion levels vary over time, with particular focus on diel trends, and how they vary directionally, i.e., between horizontal and vertical axes. Correlations between diel particle motion trends and visual indicators of habitat quality were also investigated, as have been reported previously for sound pressure data (Kaplan et al., 2015). Particle motion levels of example fish sounds and boat noise were also described. Finally, we discuss research questions regarding reef soundscapes that would necessitate particle motion measurement, and those that may only require descriptions of sound pressure.

\section{METHODS}

\section{Study sites and visual surveys}

Study sites were along the southern shore of St. John, U.S. Virgin Islands (18.31' N, 64.74' W), within the Virgin Islands National Park (Fig. 1A). Four reef sites that represent a range of habitat quality were selected for this study: Tektite, Yawzi, Ram Head, and Cocoloba. 
Visual surveys of benthic cover and fish presence were conducted by SCUBA divers from July 17-24, 2017. For detailed methods of visual surveys, see Dinh et al. (2018) and Kaplan et al. (2015). Briefly, benthic point surveys were conducted along six 10-m transects, with data recorded at $10 \mathrm{~cm}$ increments, to quantify percentages of different types of benthic cover including hard and soft corals, macroalgae, sponges, sand, and rock. For the present study, we focused on coral cover as a benthic habitat quality indicator. The number of points identified as hard and soft coral were totaled and divided by the total number of points surveyed at a site $(n=600)$, and reported as percent coral cover. Fish surveys consisted of three $30-m$ video transects per site. These transects started at the location of the acoustic array, and were swum straight along haphazardly selected bearings. Tektite and Yawzi had higher percent coral cover and greater fish abundance than Ram Head and Cocoloba (Table 1). Tektite also had the highest species richness (number of different fish species) whereas Cocoloba had the lowest species richness. Overall, surveys indicated Yawzi and Tektite were healthier reefs (i.e., with more coral cover and supporting more fish or higher biodiversity) during the study period compared to Ram Head and Cocoloba.

\section{Passive acoustic array configuration}

At each reef, a four-channel array was deployed on a rebar stake, $1 \mathrm{~m}$ above the substrate. The recorders were at the following depths: Tektite: 10.6 m, Yawzi: 9.1 m, Ram Head: 8.5 m, Cocoloba: 7 m. Arrays consisted of four hydrophones (HTI-96-MIN/3V/Low Noise; High Tech Inc.; nominal sensitivity: $-165 \mathrm{~dB}$ re $1 \mathrm{~V} / \mu \mathrm{Pa}$; frequency response: $2 \mathrm{~Hz}$ to $30 \mathrm{kHz}$ ) secured to a PVC frame and spaced $0.38 \mathrm{~m}$ apart in an orthogonal arrangement (Fig. 1B). Hydrophones were connected to a SoundTrap ST4300 (Ocean Instruments, NZ), which synced recordings across channels, applied a $4 \mathrm{~dB}$ gain, and digitized each channel at a $48 \mathrm{kHz}$ sample rate.

\section{Recording schedule}

Recordings were collected between March 19 and November 21, 2017. All SoundTraps were set to a duty cycle of $63 \mathrm{~s}$ per $10 \mathrm{~min}$. Each file had a $3 \mathrm{~s}$ "ramp-up" (a DC offset caused by the SoundTrap) which was omitted from analyses, leaving $60 \mathrm{~s}$ available for each recording. 
Hurricanes Irma and Maria made landfall at St. John on September $6^{\text {th }}$ and September $20^{\text {th }}$ respectively, causing data loss between August and November for Yawzi and Ram Head. Arrays at Tektite and Cocoloba survived, however they were thrown off their rebar stakes, likely during Hurricane Irma. Upon recovery in November, these two arrays were found lying on their side on the sand 10-20 $\mathrm{m}$ away from their original locations. Although recovered long-term data are shown for the full deployments in Figure 2, due to array displacement and consequently a lack of comparability of post-hurricane recordings with earlier recordings, subsequent analyses for all sites were limited to March through August 2017.

\section{Acoustic analyses}

All acoustic data analyses were conducted in MATLAB versions 2016b and 2020a. A random $3 \mathrm{~s}$ sample within each $60 \mathrm{~s}$ recording was used to calculate all metrics. Though acoustic integration times of sound perception by invertebrates (and fishes) are largely unknown, this $3 \mathrm{~s}$ integration time was considered more representative of individual biological signals (which can vary in duration but are relatively short) and of how these taxa may integrate sounds, compared to a 60 s integration. Particle acceleration was calculated using the finite difference approximation, which is based on the pressure gradient between a pair of hydrophones:

$$
a_{21}(t)=-\frac{\left(p_{2}(t)-p_{1}(t)\right)}{\rho d},
$$

where $p_{1}(t)$ and $p_{2}(t)$ are the pressures (Pa), at two hydrophones at time point $t, \rho$ is the seawater density (1022 $\mathrm{kg} \mathrm{m}^{-3}$; average of CTD casts at all sites during summer 2017), $d$ is the distance $(0.38 \mathrm{~m})$ between hydrophones, and $a$ is the particle acceleration $\left(\mathrm{m} \mathrm{s}^{-2}\right)$ along the axis of the two hydrophones. Particle acceleration was calculated along three axes, with hydrophones 4 and 3 forming the $X$ axis, 4 and 2 forming the $Y$ axis, and 4 and 1 forming the vertical $\mathrm{Z}$ axis (as labeled on Fig. 1B).

Root-mean-square (rms) levels were calculated for pressure and particle acceleration in a $100-1000 \mathrm{~Hz}$ frequency band after filtering data with an $8^{\text {th }}$ order Butterworth filter. These quantities are hereafter referred to as $S P L_{r m s}$ (sound pressure level; units: $d B$ re $1 \mu \mathrm{Pa}$ ) and PALrms (particle acceleration level; units: $d B$ re $1 \mu \mathrm{m} \mathrm{s}^{-2}$ ), using reference units set by ISO 
standards (ISO/DIS, 2017). The 100-1000 Hz band was selected because it covers much of the hearing ranges of invertebrates and fish, while limiting errors of calculated particle acceleration inherent in the array setup (signal to instrument-noise ratio, calibration and spacing uncertainty error), which were expected to be relatively greater outside of this frequency range (Appendix D: Fig. S1; Gray et al., 2016). Sliding daily averages of PAL $L_{r m s}$ and $S P L_{r m s}$ were calculated to observe long term trends over the entire deployment at each site.

To investigate diel cycles of soundscape data, dusk periods were defined from sunset to 90 min after sunset, and dawn periods were defined from 75 min before sunrise to sunrise, reflecting astronomical twilight periods year-round. Long-term periodograms were plotted to visualize the relative strength of diel periodicity, using Welch's method with a sample rate of 144 samples per day (corresponding to the recorder's duty cycle), a FFT size of 2880 samples, a window length of 20 days, and $50 \%$ overlap of time windows.

All data were manually scanned for the presence of boat noise by looking at pressure spectrograms generated for each audio file, following Dinh et al. (2018) and Kaplan \& Mooney (2015). Except where otherwise noted, results reported are from recordings without boat noise, representing natural sound sources on the reefs.

Spectral analyses encompassed frequencies from 5 to $2000 \mathrm{~Hz}$ to allow a wider range to compare soundscape data with particle-motion detection thresholds of fishes and invertebrates. Power spectral density (PSD) was calculated using Welch's method, in $1 \mathrm{~Hz}$ bins and over $1 \mathrm{~s}$ time windows with 50\% overlap. Units for PSD were $\mathrm{dB}$ re $1 \mu \mathrm{Pa}^{2} \mathrm{~Hz}^{-1}$ and $\mathrm{dB}$ re (1 $\left.\mu \mathrm{m} \mathrm{s}^{-2}\right)^{2} \mathrm{~Hz}^{-1}$ for pressure and particle acceleration, respectively. For each recording, peak PSD and frequency were extracted for comparison with audiograms. One octave band levels were calculated at center frequencies of $16,32,63,125,250,500$, and $1000 \mathrm{~Hz}$, via octave smoothing of PSD data using the poctave function from MATLAB's Signal Processing Toolbox. Spectrograms were also plotted for 1 min examples of boat noise and 4-30 s examples of fish sounds (selected from files within the $90^{\text {th }}$ percentile of PAL $r m s$ ), in $8 \mathrm{~Hz}$ bins and $125 \mathrm{~ms}$ time windows with $80 \%$ overlap. 
The rms and PSD metrics were calculated for each particle acceleration axis $(X, Y, Z)$. They were also calculated as a vector (Euclidean) norm to report an overall 3D magnitude, as follows:

$$
a_{T o t}=\sqrt{a_{x}^{2}+a_{y}{ }^{2}+a_{z}{ }^{2}},
$$

where $a_{i}$ represents either the rms or PSD value of particle acceleration obtained for an individual axis $i$. The mean pressure of all four hydrophones was taken when comparing pressure with 3D particle acceleration.

The PSD of 3D particle acceleration obtained from Equation 6 was also compared with PSD of theoretical particle acceleration predicted for a plane wave in the far field, calculated as follows:

$$
a_{p w}=\frac{2 \pi f * \sqrt{P_{P S D}}}{\rho c},
$$

where $f$ is the frequency $(\mathrm{Hz}), P_{P S D}$ is the mean PSD (for each $1 \mathrm{~Hz}$ bin) of all four hydrophones $\left(\mathrm{Pa}^{2} \mathrm{~Hz}^{-1}\right), \rho$ is the seawater density $\left(\mathrm{kg} \mathrm{m}^{-3}\right), c$ is the sound speed $\left(1543 \mathrm{~m} \mathrm{~s}^{-1}\right.$; as measured via CTD data), and $a_{p w}$ is the particle acceleration for a plane wave in the far field $\left(\mathrm{m} \mathrm{s}^{-2}\right)$. The $a_{p w}$ was then converted to PSD in $\mathrm{dB}$ re $\left(1 \mu \mathrm{m} \mathrm{s}^{-2}\right)^{2} \mathrm{~Hz}^{-1}$. 3D particle acceleration from Equation 6 close to or below that from Equation 7 can be approximated as a plane wave, whereas higher acceleration levels from Equation 6 indicate sounds propagating as different wave types, such as point sources, or sounds recorded in the near field (Gray et al., 2016; Rogers \& Cox, 1988).

To place soundscape levels in the context of animal hearing abilities, as done in previous studies (Amoser \& Ladich, 2005; Mooney et al., 2018), we compared previously published hearing thresholds to PSD levels ( $1 \mathrm{~Hz}$ bins) and wider bands (1 octave in the present study), with the latter approximating hypothesized auditory frequency filtering by marine fishes, such as cod (Hawkins \& Chapman, 1975; Stanley et al., 2017); no such filtering estimates have been made for aquatic invertebrates. Very few studies report fish and invertebrate particle motion thresholds (Popper \& Hawkins, 2018), and none exist for Caribbean species; thus we compared thresholds of species from other habitats to give a general indication of these animals' abilities to detect the soundscape. 


\section{Statistical analyses}

Correlations between wide-band $(100-1000 \mathrm{~Hz}) \mathrm{SPL} \mathrm{L}_{\mathrm{rms}}$ and 3D PAL $\mathrm{L}_{\mathrm{rms}}$, and between 1D axes of PALrms, were analyzed with ordinary least squares regression and Spearman's Rho. To assess the influence of boat noise on particle motion levels, Mann-Whitney $U$ tests were performed to test differences in the distribution of $\mathrm{PAL}_{\mathrm{rms}}$ between files with and without boat noise at each reef site $(\alpha=0.05)$. To quantify the strength of diel particle motion trends, medians across each time of day were found for the whole analysis period (March through August) for each site. Then, the maximum of the median levels during dawn or dusk periods (where medians were highest throughout a day) was subtracted by the minimum median level during the night (where medians were lowest throughout a day). These dawn-night and dusknight differences were regressed against percent coral cover and fish abundance data for each site, and Pearson correlation coefficients were reported $(\alpha=0.05)$.

\section{RESULTS}

Long-term trends in particle acceleration and pressure

At all sites, daily averages of $1 D P A L_{r m s}$ and $S P L_{r m s}$ gradually increased from April to August by ca. $3 \mathrm{~dB}$ (Fig. 2). On this temporal scale, trends of the three particle acceleration axes and sound pressure closely matched each other. Strong peaks of abiotic sounds (wind, wave motion and rain) in both PALrms and SPL $L_{r m s}$ occurred in September when hurricanes Irma and Maria made landfall at St. John. Peaks at Cocoloba from October 14-25 were likely due to the hydrophones brushing against the benthos or animals brushing against the hydrophones repeatedly, leading to noise artefacts. Prior to hurricanes, at all sites but Yawzi these averages were about 1-3 dB lower in the $\mathrm{Z}$ axis compared to the horizontal axes, whereas the horizontal axes were within $1 \mathrm{~dB}$ of each other. At Yawzi, all three axes had $P A L_{r m s}$ within $1 \mathrm{~dB}$ of each other, though the $\mathrm{Z}$ axis was consistently higher than $\mathrm{X}$ and $\mathrm{Y}$.

Including all recordings from March through August, 3D PALrms had moderate correlation with SPLrms at Tektite and Yawzi $\left(R^{2}=0.74\right.$ and 0.76 , respectively), and weaker correlation with sound pressure at Ram Head and Cocoloba $\left(R^{2}=0.50\right.$ and 0.60 , respectively, 
Fig. 3). Correlations were statistically significant $(p<0.001)$ for each site. At each site there were two overlapping clusters in these scatterplots; 3D PAL $\mathrm{L}_{r m s}$ above $45 \mathrm{~dB}$ and $S P \mathrm{~L}_{r m s}$ above $100 \mathrm{~dB}$ appeared to cluster around a steeper slope than that below these values (Fig. 3). These clusters were also distinguished with residual plots (Fig. S2), which showed residuals within \pm 10 $\mathrm{dB}$ at lower $S P L_{r m s}$ but strong divergence of $P A L_{r m s}$ (residuals $>+15 \mathrm{~dB}$ ) from the regression line at SPL $L_{r m s}$ of $95-100 \mathrm{~dB}$ and higher. This could indicate the presence of several types of acoustic fields recorded at these sites (see discussion).

Quantiles ( $25^{\text {th }}$ to $99^{\text {th }}$ ) of 3D PSD for empirical particle acceleration from Equation 6 were compared to PSD for theoretical particle acceleration calculated for a far field plane wave (Equation 7). Above $100 \mathrm{~Hz}$, empirical PSD levels at most of these quantiles closely approximated those of a plane wave (Fig. S3). The $99^{\text {th }}$ percentile empirical curve for Tektite was at least $6 \mathrm{~dB}$ greater than the respective plane wave curve from 100-300 Hz, peaking at 10 dB greater at $300 \mathrm{~Hz}$. From 2-100 Hz, empirical acceleration PSD remained relatively flat whereas theoretical acceleration logarithmically increased with increasing frequency; theoretical plane wave acceleration was as much as $40 \mathrm{~dB}$ re $1\left(\mu \mathrm{m} \mathrm{s}^{-2}\right)^{2} \mathrm{~Hz}^{-1}$ lower than empirical acceleration. Thus the plane-wave approximation greatly under-predicted true particle acceleration of the soundscape in this low frequency range. Examples of these comparisons for Ram Head and Tektite are shown in Fig. S3.

\section{Diel patterns and diversity of reef sounds}

All sites were similar in their diel periodicity, with higher 3D PALrms levels during the day than at night, and peaks during dawn and dusk (Fig. 4; examples shown for Tektite and Cocoloba). This pattern was also true for the three axes of particle acceleration and for pressure (Fig. S4). Periodograms also indicated diel periodicity, peaking at one cycle per day and per multiples of one day. Diel cycles were strongest at Tektite, followed by Yawzi, Ram Head, and weakest at Cocoloba. These diel patterns primarily reflect fish sounds, with higher amplitude tonal chorusing of multiple individuals detected in crepuscular periods, as observed previously (Kaplan \& Mooney, 2015). Compared to other sites, at Tektite quantiles of PALrms were more variable between adjacent times of day, especially the $90^{\text {th }}$ percentile. The peak shortly after 
dawn in the $90^{\text {th }}$ percentile curve for Cocoloba was due to loud broadband fish sounds like those shown in Fig. 5a (from Tektite); these sounds occurred at other times of day as well.

Recordings from Tektite and Cocoloba with 3D PAL rms $_{\text {with }}$ the $90^{\text {th }}$ percentile were sampled, and among these a wide diversity of pulsed and tonal of fish sounds were found (Fig. 5). Our study aimed to provide an overview of particle motion of different fish sounds, rather than identify sounds to specific taxa. Any such identification is speculative at this time due to lack of a comprehensive database of Caribbean reef fish sounds, and lack of synced visual and audio data that would allow identification of sound producers. The greatest particle acceleration peaks seen in the $90^{\text {th }}$ percentile curves in Fig. 4 (> $55 \mathrm{~dB}$ PAL $\mathrm{L}_{\mathrm{rms}}$ ) were from broadband, "grinding" pulses at short (<1s) intervals, which are likely stridulatory or feeding sounds (e.g., grinding of pharyngeal teeth against each other or when feeding on coral) from unidentified fishes (Fig. 5a). Other fish sound types included trains of frequency down-sweeps from 200 to $100 \mathrm{~Hz}$ (Fig. 5b,j), rapid broadband pulses with peak frequencies around $500 \mathrm{~Hz}$ (Fig. 5c), short broadband pulses with peak frequencies between 200-400 Hz (Fig. 5e,h), pulsed calls peaking around $50 \mathrm{~Hz}$ with a broadband component at the beginning of the pulse (Fig. 5e), short (< 0.5 s) tonal "groans" with a fundamental frequency near $100 \mathrm{~Hz}$ (Fig. 5f), a crepuscular chorus of tonal $1 \mathrm{~s}$ duration calls between $400-500 \mathrm{~Hz}$ (Fig. $5 \mathrm{~g}$, shown during dusk), broadband calls with more diffuse energy across time and frequencies (Fig. 5i), and rapidly-pulsed calls with harmonics at 400 and $800 \mathrm{~Hz}$ (Fig. 5I). Examples of dusk choruses with multiple types of these calls together are shown in Figure $5 d, 5 k$. Visual surveys identified a variety of fish belonging to known soniferous taxa (Amorim, 2006; Kaschner, 2012; Tricas \& Boyle, 2014) which may contribute to these sounds, including but not limited to Caranx spp. (jacks), Haemulon spp. (grunts), Holocentridae (squirrelfish and soldierfish), Lutjanus apodus (schoolmaster snapper), Ocyurus chrysurus (yellowtail snapper), Pomacentridae (damselfish), Scaridae (parrotfishes), Sciaenidae (drums/croakers), and Serranidae (groupers).

\section{Boat noise}

There was substantial overlap in the distribution of 3D PAL ${ }_{r m s}$ between recordings without boat noise and recordings with boat noise (Fig. 6a). This overlap may be attributed to 
the fact that many recordings had boat noise at levels close to background ambient noise (likely from more distant ships) in addition to those with louder boat noise (likely close-passing boats), and the fact that many recordings without boat noise contained loud fish sounds (Fig. 5), presumably within short distances (e.g., several meters) from the array. Median levels were significantly higher for boat noise at each reef site $(p<0.001$, Mann-Whitney $U$ tests). Example 3D particle acceleration spectrograms of individual boat passes are shown recorded at Tektite (Fig. 6b) and Yawzi (Fig. 6c). Spectrograms of individual boat passes were similar in sound pressure and 3D particle acceleration. Those of individual particle acceleration axes could vary slightly across time; these differences likely were due to the relative direction of travel of the boat relative to the hydrophone array, where they may approach and leave a reef site along different axes (Fig. S5).

Particle motion of reef soundscapes relative to fish and invertebrate hearing thresholds

Plots of peak PSD from 5-2000 Hz revealed clusters of data points around different frequency bands that correspond to different soundscape components (Fig. 7). Above $1000 \mathrm{~Hz}$, peaks primarily are from snapping shrimp snaps. Peak PSD levels around $400-800 \mathrm{~Hz}$ likely are from various types of fish sounds, such as those seen in Fig. 5c, 5g, and 5i, whereas the cluster around $300 \mathrm{~Hz}$ may correspond to fish sounds seen in Fig. $5 f$ and $5 \mathrm{~h}$. Peaks below $100 \mathrm{~Hz}$ likely correspond to broadband fish sounds with low-frequency peaks (Fig. 5a,5e) as well as abiotic wind and wave noise (as seen in Fig. $5 \mathrm{~g}$ ).

Peak particle acceleration PSD levels were compared with previously published particle acceleration audiograms of several invertebrate species (Fig. 7a) and fish species (Fig. 7b). None of these species inhabit Caribbean reefs and only one of the fish species shown inhabits tropical coral reefs (Chiloscyllium plagiosum; Indo-Pacific). No particle motion sensitivity data have been published for fishes or invertebrates native to Caribbean reefs. The species selected cover a range of sensitivities and give a comparative view to broadly investigate detectability of ambient coral reef particle motion by these taxa. At $100 \mathrm{~Hz}$ and above, even the highest particle acceleration PSD levels were below hearing thresholds of invertebrates, and only a few data points were above thresholds of fishes, including Sciaena umbra (brown meagre), 
Micropogonias unduluatus (Atlantic croaker), and Chiloscyllium plagiosum (white-spotted bamboo shark). Below $80 \mathrm{~Hz}$, multiple data points were above the hearing thresholds of some invertebrates, including the mussel Mytilus edulis and the hermit crab Pagurus bernhardus. Among fishes whose particle acceleration thresholds were measured below $80 \mathrm{~Hz}$, C. plagiosum had the lowest thresholds, and peak PSD levels of the soundscape were up to $60 \mathrm{~dB}$ higher, suggesting hearing of these cues was likely. Notably, C. plagiosum was the only of these species whose audiogram was obtained using a dipole sound projector (discussed further below). Comparing sound pressure-derived audiograms of fishes known to detect sound pressure, the highest outliers of the soundscape between $100-600 \mathrm{~Hz}$ reached or exceeded hearing thresholds (Fig. 7c). Yet, the majority of peak PSD pressure values were at least $10 \mathrm{~dB}$ below the pressure hearing thresholds, suggesting hearing of these pressure cues may be limited as well. Another way to compare soundscape data with hearing data is to report soundscape data in frequency bands approximating auditory filters of species of interest, which is appropriate when estimating the detectability of signals (e.g. fish calls) among ambient noise (Stanley et al., 2017). Again, the bandwidth of auditory filters has not been determined for any marine invertebrate, though one octave bands have been used to approximate those of fishes (Hawkins \& Chapman, 1975; Stanley et al., 2017). Percentiles of one octave levels during dawn at Tektite (these percentiles were nearly identical for dusk), and examples of fish and boat noise in the $99^{\text {th }}$ percentile at Tektite were compared with audiograms (Fig. 8). The $90^{\text {th }}$ and $99^{\text {th }}$ percentiles of natural ambient levels, and $99^{\text {th }}$ percentiles of fish sounds and boat noise reached or exceeded particle acceleration thresholds of the two most sensitive fish species shown in Figure 7b. These thresholds were still above median ambient octave band levels.

\section{Particle motion trends as indicators of habitat quality}

Strength in the diel trend of 3D PAL $L_{r m s}$ (dawn or dusk levels relative to night levels) was significantly correlated with percent coral cover ( $p<0.05$, Fig. 9), but not significantly correlated with fish species richness and fish abundance. With four data points in these analyses, statistical power was low. Regression coefficients indicated moderate-to-strong correlation for all metrics ( $R^{2}$ range: $\left.0.72-0.93\right)$. Diel strength of $S P L_{r m s}$ had significant 
correlation with percent coral cover $\left(p<0.05\right.$, dawn $R^{2}=0.96$, dusk $\left.R^{2}=0.98\right)$ and fish abundance $\left(p<0.05\right.$ for dawn only, $\left.R^{2}=0.98\right)$, and weaker correlation with fish species richness $\left(R^{2}=0.58-0.82\right.$, Fig. S6). For each site, 3D PAL $L_{r m s}$ and $S P L_{r m s}$ diel trend strengths were similar, within $2 \mathrm{~dB}$ of each other.

\section{DISCUSSION}

Our dataset revealed important relationships between particle acceleration and pressure at shallow coral reefs. We found parameters at which these metrics led to similar results, and at which empirical acceleration diverged from common theoretical approximations. Empirical acceleration and pressure usually correlated well with one another and had similar diel and long-term trends. However, particle acceleration was greater relative to pressure (and relative to acceleration predicted for plane waves) for high amplitude and low frequency signals (Fig. 3, Appendix D: Fig. S3). We found subtle directional differences in particle acceleration; averaged over time, particle acceleration levels were higher in the horizontal axes than the vertical axis at most sites. Ambient particle acceleration levels, and those of outlier (high amplitude) fish and boat sounds were often below particle acceleration detection thresholds reported for invertebrates and for many fish, suggesting limited detectability of reef sounds by these taxa. Lastly, we found that the strength of diel trends in particle acceleration and pressure significantly correlated with visual habitat quality indicators such as coral cover.

\section{Particle acceleration relationships with sound pressure}

As seen in the regression plots (Fig. 3; Appendix D, Fig. S2) root-mean-square particle acceleration levels positively correlated with sound pressure levels, although these data appeared to have two overlapping clusters around slightly different slopes. The lower amplitude cluster $\left(\mathrm{PAL}_{\mathrm{rms}}<45 \mathrm{~dB} ; \mathrm{SPL}_{\mathrm{rms}}<100 \mathrm{~dB}\right)$ likely included sounds more closely approximating plane wave propagation in the acoustic far field. The higher amplitude cluster $\left(\mathrm{PAL}_{\mathrm{rms}}>45 \mathrm{~dB} ; \mathrm{SPL}_{\mathrm{rms}}>100 \mathrm{~dB}\right)$, might include more sounds recorded in the near field, for example fish vocalizing within a few meters of the array, where a higher ratio of particle motion 
to pressure is expected (Gray et al., 2016; Rogers \& Cox, 1988). Relative magnitudes of particle motion (velocity or acceleration) over distance in the near field depend on the type of acoustic field (e.g., monopole, dipole, multipole) and its frequency content (Kalmijn, 1988; Popper \& Hawkins, 2018). Such "near field effects" may have contributed to higher particle acceleration than predicted for far field plane waves, as seen for higher amplitude sounds ( $>45 \mathrm{~dB}$ re $1 \mu \mathrm{m} \mathrm{s}^{-}$ 2, Fig. 3) and at lower frequencies ( $100 \mathrm{~Hz}$, Fig S3). As estimated by Equation 2 (Chapter 1) using typical density and sound speed values for sandy substrate (Nedelec et al., 2016); our acoustic recorders were over sandy patches within a reef), the cutoff frequencies were below $100 \mathrm{~Hz}$ for each site (as low as $47 \mathrm{~Hz}$ for Tektite and as high as $71 \mathrm{~Hz}$ for Cocoloba, depending on site depth). Consistent with theory, below these cutoff frequencies empirical particle acceleration was much greater than that predicted for plane waves. Thus, the relatively shallow depths of the reef sites $(<11 \mathrm{~m})$ may have also contributed to higher particle motion to pressure ratios than those predicted for plane waves. Differences between empirical and theoretically estimated particle motion may also arise from the directional nature of many fish and other natural sounds, which tend to propagate as dipoles or higher-moment (multipole) fields with variable magnitudes of particle motion along different spatial axes (Kalmijn, 1988). Overall, our soundscape data show that using the plane-wave approximation (e.g., from single pressure measurements) will underestimate particle motion magnitudes of many sounds present on coral reefs.

Studies correlating empirically measured particle motion and pressure in the field are rare. Similar to the present study, a two day study in a shallow ( $8 \mathrm{~m}$ deep) bay in Brazil found that dawn and dusk chorus patterns were present in particle velocity and pressure data; however, near crepuscular periods particle velocity and acceleration had low-frequency $(<120$ $\mathrm{Hz}$ ) peaks not present in pressure data (Jesus et al., 2020). A study recording pile driving sounds within a shallow harbor (2.5 $\mathrm{m}$ depth) found that magnitudes of horizontal and vertical particle velocity were significantly correlated with sound pressure (with $R^{2}>0.62$ ), although all particle velocity levels were above those predicted by theory for a plane wave in the far field (Ceraulo et al., 2016). Similarly, in shallow streams (<1 $\mathrm{m}$ depth) particle velocity to pressure ratios were greater than those expected for plane waves at sites with relatively high ambient noise, from 
50-100 Hz (Lugli \& Fine, 2007). These results are expected for relatively low frequencies and shallow environments, and for sounds recorded close to boundaries or close to the sound source (Horch \& Salmon, 1973; Jesus et al., 2020; Nedelec et al., 2016). Such conditions are often met on coral reefs.

Conversely, longer-term daily trends (Fig. 2) and diel trends (Fig. 4), were similar when quantified in either particle acceleration or sound pressure. Therefore, fishes and invertebrates experience the same long-term trends and diel cycles in particle acceleration levels as those we observe in sound pressure. Accordingly, sound pressure is likely sufficient in describing temporal trends of coral reef soundscapes (discussed further below).

\section{Particle motion of fish sounds: temporal and spectral variability}

Diel trends of sound pressure were consistent with those of prior studies that reported data from the same sites (Kaplan et al., 2015; Lillis, Apprill, et al., 2018). Tektite had the strongest crepuscular peaks of particle acceleration and sound pressure, also consistent with sound pressure data at Tektite over the same months in 2013 (Kaplan et al., 2015). These peaks may reflect the fact that Tektite had the highest fish abundance and species richness of all sites. Higher sound levels in this frequency band may be due to a greater number of fish vocalizations or louder fish at these sites, or a higher number of soniferous species, though these have not been quantified. At coral reefs in Maui, Hawaii, low-frequency $(50-1200 \mathrm{~Hz})$ sound pressure was significantly positively correlated with soniferous fish abundance (Kaplan et al., 2018). The reason for the higher variance in diel levels at Tektite is unknown, but this could be attributed to a higher diversity in soniferous species, greater number of different types of fish sounds, or variability in distance of sound-producing fish from the acoustic array. Additional work is needed to investigate these phenomena.

\section{Directionality of particle motion}

For three of the reef sites, the vertical axis had slightly lower particle acceleration on average. This result is logical considering many reef fishes and invertebrates reside at or near the benthos, thus sounds produced by them may be expected to propagate at angles closer to 
horizontal than vertical with respect to the array. The acoustic array at Yawzi was surrounded by more vertical reef structure than arrays at other sites, which may explain why the vertical particle motion axis at that site was about equal to or slightly higher than the horizontal axes. Directional differences in underwater particle motion have previously been tied to nearby benthic structure and the presence of rocky boundaries (Jesus et al., 2020). On the other hand, directional differences in long-term ambient particle acceleration were relatively small (within 3 $d B$, Fig. 2), suggesting that on average, sounds were coming from many directions with respect to the array. Though particle motion fields likely carry ecologically significant directional cues for invertebrates and fishes (Wilson et al., 2018; Zeddies et al., 2012), the directionality of individual, specific cues (e.g., fish calls) may be more relevant than that of average ambient levels.

What aspects of the particle motion soundscape are detectable by fishes and invertebrates?

Our data suggest invertebrates and many fishes could rarely detect natural soundscape cues or boat noise present on these reefs, especially above $100 \mathrm{~Hz}$. This finding is consistent with particle acceleration measurements directly above reefs in Maui, Hawaii, which were maximal at $30 \mathrm{~dB}$ re $1 \mu \mathrm{m} \mathrm{s}^{-2}$ from $200-300 \mathrm{~Hz}$, and below published particle acceleration thresholds of many species except the Atlantic Croaker M. unduluatus (Kaplan \& Mooney, 2016). Pressure-derived hearing thresholds of butterflyfish (native to the Indo-Pacific) also are above the ambient levels of their reef habitats from $100-1000 \mathrm{~Hz}$, but the sound pressure of some signals produced by conspecifics slightly exceed hearing thresholds (Tricas \& Webb, 2016). These butterflyfish predominantly sense particle motion over sound pressure, yet their particle acceleration thresholds from $100-1000 \mathrm{~Hz}$ are still above the maximum values recorded on St. John and Maui reefs [ibid]. A recent study compared particle motion audiogram data of multiple species to soundscape data from coral reefs (as shallow as $9 \mathrm{~m}$ ) at Moorea Island, French Polynesia; the authors estimated detection distances up to $13 \mathrm{~m}$ for fishes except for Sciaena umbra (up to $430 \mathrm{~m}$ ), and limited detection distances of 1-59 m for invertebrates except for the prawn Palaemon serratus (up to 195 m) (Raick et al., 2021). However, that study made comparisons by converting particle-motion derived thresholds to pressure using the 
plane wave approximation, an estimation that did not hold up in our empirical data. Several temperate, coastal Mediterranean fish species have sound pressure hearing thresholds 20-60 dB higher than natural ambient noise recorded in their habitats (Codarin et al., 2009).

Conversely, pressure-based hearing thresholds of freshwater fish including roach (Rutilus rutilus) and common carp (Cyprinus carpio) are below ambient pressure levels in their habitats, but particle acceleration audiogram data are limited or absent for these species (Amoser \& Ladich, 2005; Magnhagen et al., 2017).

Though determination of communication and detection distances was not a goal of our study, our results reinforce prior studies that estimate short communication and detection distances of fish calls, such as those of damselfish and oyster toadfish, at only 5-10 m away (Higgs \& Radford, 2016). Our results also reinforce the idea that particle motion cues from coral reefs are likely of limited use to fishes and invertebrates for navigation toward reefs, as discussed in Kaplan \& Mooney (2016). For the present study we lack data to determine the distance of fish calls and other sounds from the array. Further, we lack empirical data on the distances over which marine invertebrates can detect particle motion of ecologically relevant cues. However, theory suggests many invertebrates may only detect particle motion of coral reef sounds within meters of the source (Raick et al., 2021). Importantly, our baseline dataset provides empirical measurements of particle acceleration levels of ambient reef noise, which can be leveraged in future studies investigating detection distances of particle motion cues for reef inhabitants.

It may be advantageous for coral reef fishes and invertebrates to be unable to detect lower amplitude, ambient reef sounds. They may primarily need to detect sounds from conspecifics or other species at close range and higher amplitudes, e.g., for communication or detecting predators or prey. The "background" biological cacophony of ambient noise on reefs may be less ecologically relevant. Essentially, these animals might "filter" cues out from the noise by only detecting higher sound levels, i.e., by having relatively high hearing thresholds. This way, their detection of nearby sounds above hearing thresholds would be less prone to masking from ambient noise. This phenomenon has been demonstrated in several fish species (Ladich, 2019; Wysocki \& Ladich, 2005). 
Although the present results are consistent with prior studies reporting ambient particle acceleration levels below fish and invertebrate thresholds, there are many challenges and unknowns in making these comparisons, which preclude concluding with certainty that invertebrates and fish could rarely detect particle motion cues of these reef soundscapes. These include: 1) limitations and differences in methods used to collect audiograms, and 2) unknowns regarding how invertebrates and fishes perceive particle motion.

Generally, fishes and invertebrates are most sensitive to particle motion at frequencies below 100 Hz (Packard et al., 1990; Sand \& Karlsen, 2000), where soundscape data from St. John reefs had the highest peak PSD values. Thus, soundscape cues in this range are more likely detectable and therefore more likely ecologically relevant for these animals. Fishes are thought to rely on particle motion more at lower frequencies to detect signals, and pressure more at higher frequencies (Ladich \& Fay, 2013; Wysocki et al., 2009). However, audiogram data for most of these species has only been obtained as low as $80-100 \mathrm{~Hz}$ due to methodological limitations such as the limited frequency output range of commonly used underwater speakers (Tricas \& Webb, 2016). Thus there is a need for more studies addressing lower frequencies of hearing thresholds. Also, species inhabiting St. John and Caribbean reefs may have different particle motion sensitivities than the species presented here, which inhabit other regions.

There are technical limitations of many audiogram studies that may lead to higher measured thresholds (lower sensitivity) than the "true" thresholds of animals. First, audiograms measured via neurophysiological methods tend to be higher than those measured via behavioral responses, sometimes by $20 \mathrm{~dB}$, as demonstrated in some fishes (Kojima et al., 2005). Most of the audiograms shown in Figure 7 are based on neurophysiological data (AEPs). Second, although researchers try to minimize ambient noise to determine "absolute" sensitivities, thresholds can vary with different experimental noise floors. Elevated background noise has been shown to raise measured hearing thresholds in several fish species (Amoser \& Ladich, 2005; Popper et al., 2019; Wysocki \& Ladich, 2005). Third, many hearing studies use monopole speaker setups, whereas natural sounds such as fish calls may be more dipole or multipole, and thus more highly directional (Kalmijn, 1988; Zeddies et al., 2012). For example, white-spotted bamboo sharks had lower hearing thresholds for dipole stimuli compared to 
monopole stimuli; this difference hypothetically arose due to different hearing structures being stimulated by each acoustic field type (Casper \& Mann, 2007). Fourth, most thresholds are obtained by playing artificial tone bursts which may stimulate invertebrate and fish sounddetection structures differently than more spectrally and temporally complex natural cues (Maruska \& Tricas, 2009; Wright et al., 2011; Wysocki \& Ladich, 2003).

There are many uncertainties in the neural mechanisms of how invertebrates and fishes utilize particle motion cues to perceive, locate, and respond to ecologically relevant signals. Hair cells in fish and invertebrate particle motion sensory structures are directionally sensitive and are arranged to detect motion three-dimensionally. How exactly these animals integrate particle motion cues along multiple vectors to determine the propagation direction or origin of sounds remains unclear (Budelmann, 1992a; Budelmann \& Williamson, 1994; Hawkins \& Popper, 2018). There is also poor understanding of how fishes and especially invertebrates integrate sound cues across time and frequencies, both at sensory peripheries and higher neural processing centers (Popper et al., 2019). Studies investigating these processes have primarily focused on freshwater fishes (e.g., goldfish) and terrestrial insects (Faure et al., 2008; Fay \& Passow, 1982). Hearing thresholds are usually obtained with short duration burst signals (on the order of tens of milliseconds), but measured thresholds can vary depending on stimulus duration. For example, goldfish have lower thresholds to $500 \mathrm{~ms}$ long stimuli compared to 50 ms stimuli (Popper, 1972). Further, as shown in several fishes, hearing thresholds can shift ontogenetically and with body size. Some fish species have improved hearing from juveniles to adults and with increasing size (Wright et al., 2011). Others have decreased sound sensitivity at larger sizes and later ontogenetic stages, although these changes may be non-linear (Salas et al., 2018; Stanley et al., 2020). To summarize, for more complete and accurate comparisons of invertebrate and fish hearing abilities with particle motion soundscape data, we need more detailed understanding of hearing mechanisms including directional, frequency, and temporal filtering, more measurements of behavioral responses to ecologically realistic stimuli, and more studies testing hearing of multiple life stages of given species. 


\section{Particle motion of boat noise at coral reefs}

Similar to the highest amplitude biological sounds below $1000 \mathrm{~Hz}$ (fish sounds), particle acceleration levels of the highest amplitude boat sounds were still above hearing thresholds for invertebrates and for many fish. The highest particle acceleration levels of boat noise in our study were similar to those recorded from a boat in an Australian marine reserve, which reached $40-50 \mathrm{~dB}$ re $1\left(\mu \mathrm{m} \mathrm{s}^{-2}\right)^{2}$ (Mensinger et al., 2018). This suggests many species of these taxa would have limited detection of boat noise, though they are not necessarily free from potential masking effects. The closer ambient or boat noise spectral levels are to those of ecologically relevant signals, i.e., the lower the signal-to-noise ratio (SNR), the greater the potential for masking effects (Clark et al., 2009). Several coastal Mediterranean fish species had significantly elevated hearing thresholds to artificial tones and conspecific sounds during playback of boat noise, compared to during natural ambient noise (Codarin et al., 2009). Similarly, though perch hearing thresholds were above ambient (pressure) noise of their natural habitat, thresholds were significantly higher during played ambient noise compared to quieter laboratory conditions (Amoser \& Ladich, 2005). When exposed to boat noise at similar particle motion levels to those recorded on St. John reefs, behavioral changes in multiple species have been found. For fishes, behavioral changes during boat noise have included decreased feeding activity, decreased boldness (changes in risk assessment), and decreased nest care time (Magnhagen et al., 2017; McCormick et al., 2018; Mensinger et al., 2018; Picciulin et al., 2010). Among invertebrates exposed to boat noise at similar sound levels (pressure or acceleration) observed in the present study, past studies have found increased mortality of sea hare larvae (Nedelec et al., 2014), impaired foraging and antipredator behavior of crabs (Wale et al., 2013a), and biochemical changes indicative of stress in prawns and lobsters (Celi et al., 2015; Filiciotto et al., 2016). However, particle motion was only reported in one of these studies (Nedelec et al., 2014). Due to the multitude of adverse effects observed in fishes and invertebrates when exposed to boat noise at similar amplitudes to those recorded in the present study, noise pollution from boats is still of concern for these taxa. Ideally, future studies investigating noise effects should measure particle motion and report dose-response curves to 
determine minimum particle motion magnitudes needed to elicit behavioral or physiological responses.

When is particle motion measurement necessary for soundscape studies?

When quantifying coral reef soundscapes from the perspective of invertebrates and fishes, whether or not particle motion is important to measure depends on research goals. Particle motion measurement is important when absolute (rather than relative) amplitudes of discrete and transient signals are of interest. As discussed above, particle motion of individual signals on coral reefs, especially in shallower areas, in the near field, and at lower frequencies, may have higher particle motion levels than predicted for plane waves. Thus, for accurate level data (such as to understand or predict noise impacts), actual particle motion data are needed. Particle motion measurements are also critical when assessing detectability (or masking) of soundscape components in relation to hearing abilities of fishes and invertebrates. Given reliable audiogram data, particle motion measurements can provide unique information and insight into acoustic listening and communication space.

As previously mentioned, particle motion will provide directional information about soundscape cues not present in pressure data, i.e., how these cues vary in magnitude along different spatial axes at given points in an acoustic field. Our study did not focus on quantifying directionality of particle motion of individual soundscape components (fish sounds or boat sounds), which should be investigated in future studies.

\section{Utility of particle motion in assessing habitat quality/health}

Strengths of diel particle acceleration and sound pressure trends correlated strongly with percent coral cover and fish abundance, consistent with findings for sound pressure in 2013 at these reef sites (Kaplan et al., 2015). At other tropical coral reefs, significant positive correlations have also been found between sound pressure levels at relatively low frequencies $(<2500 \mathrm{~Hz})$ and visual metrics of reef health, biomass, and biodiversity, including percent coral cover, fish density, habitat complexity, and more (Elise et al., 2019; Nedelec et al., 2015; Staaterman et al., 2017). When such trends in relative magnitudes are of interest rather than 
absolute magnitudes or directional information, reporting sound pressure may suffice (Mooney, lorio, et al., 2020).

\section{Conclusions and future directions}

This study is the first to report spatial and temporal particle motion trends of coral reef soundscapes for a duration longer than a few days, and is the first to report particle motion data at Caribbean reefs. For these relatively shallow reefs, particle acceleration levels scaled similarly with sound pressure at higher frequencies and when averaged over time, but they diverged at lower frequencies and among individual, high amplitude signals. These empirical data provide valuable new insights, and validations of concepts described in recent review papers, on the contexts in which particle motion measurement is necessary for aquatic soundscape studies, and where it may not be necessary. Particle motion measurements are essential when investigating invertebrates' and fishes' detection and utilization of soundscape cues. These data help place hearing abilities in context. Invertebrate and fish hearing thresholds were high relative to peak soundscape levels. This was surprising considering that many members of these taxa have shown phonotaxis and settlement responses to reef sounds, and produce sounds for communication. This brings into question how representative available particle motion audiogram data are of "true" hearing sensitivities. Indeed, many sources of uncertainty still exist in comparing animal hearing data to ambient soundscape data. Future development of widely-accepted standards for hearing measurements in aquatic invertebrates and fishes and for particle motion measurement, as established for mammals and sound pressure, will aid in these efforts. Baseline, long-term particle motion recordings of multiple sites, such as those from the present study, are key to solving unknowns of how and over what spatial and temporal scales reef animals utilize soundscape cues for diverse ecological

functions. Reporting particle motion of anthropogenic soundscape components is key to monitoring and predicting their impacts on invertebrates and fishes. Lastly, our observations highlight the relevance of "rare", transient, and high amplitude sounds to invertebrates and fishes over lower amplitude ambient levels. Future studies should focus on describing and 
exploring automated detection of these transients, as well as identification of the species producing them.

Table 1. Visual survey data from July 2017 for each reef site. Percent coral cover and fish abundance are shown as mean \pm standard deviation across transects. Percent coral cover includes hard and soft corals. Fish abundance is the count of individual fish, and fish species richness is the total number of species found among three transects.

\begin{tabular}{lccc}
\hline Reef Site & \% Coral Cover & Fish Abundance & Fish Species Richness \\
\hline Tektite & $28 \pm 5.2$ & $165.7 \pm 132.0$ & 36 \\
Yawzi & $23 \pm 7.1$ & $161+46.1$ & 24 \\
Ram Head & $16.2 \pm 7.3$ & $99.7 \pm 24.8$ & 27 \\
Cocoloba & $11.7 \pm 6.1$ & $51 \pm 29.2$ & 21 \\
\hline
\end{tabular}


a)

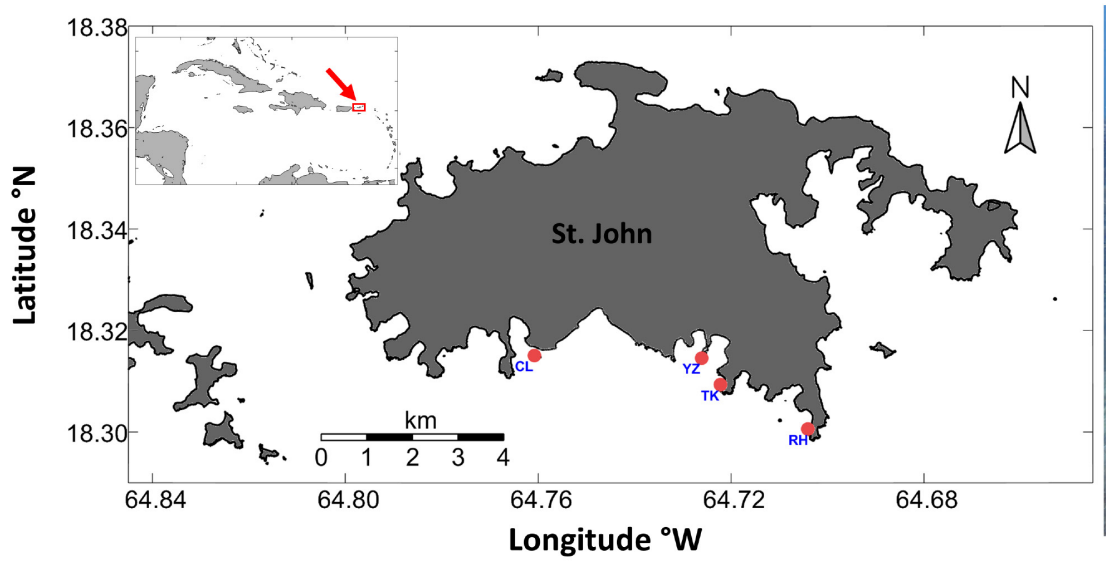

b)

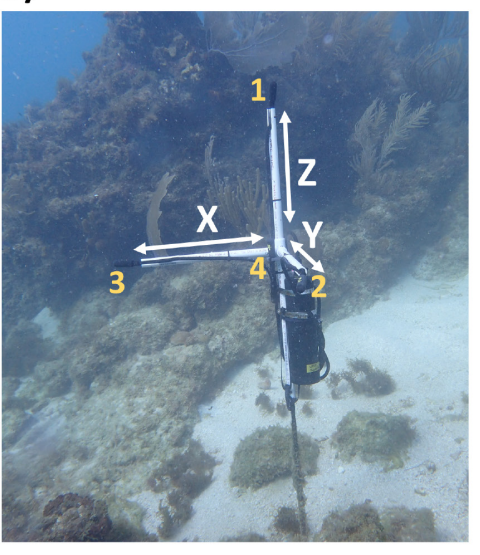

Figure 1. a) Map of St. John with reef sites marked. $C L=$ Cocoloba, $Y Z=$ Yawzi, $T K=$ Tektite, $R H=$ Ram Head. The map inset shows the location of the U.S. Virgin Islands within the Caribbean. b) Image of the hydrophone array at Cocoloba. Numbers mark locations of the four hydrophones and arrows with letters indicate the three orthogonal axes along which particle acceleration was calculated. 

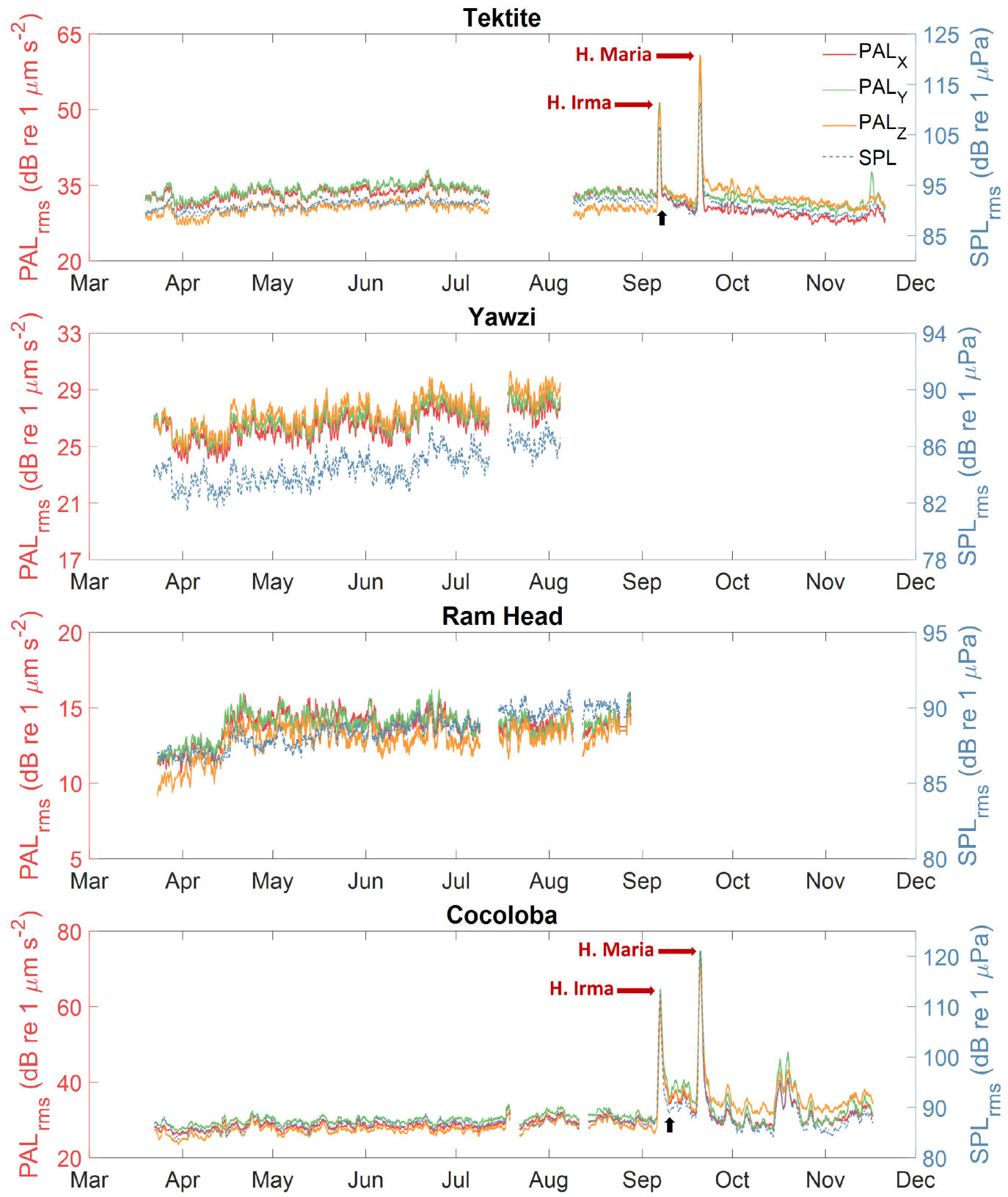

Figure 2. Sliding daily averages of root-mean-square particle acceleration ( $\left.P A L_{r m s}\right)$ along each axis $(X, Y$, Z) and sound pressure level ( $\left.S P L_{r m s}\right)$, of recordings subsampled to $3 \mathrm{~s}$ time windows and bandpass filtered to 100-1000 Hz. Peaks labeled with horizontal arrows in Tektite and Cocoloba subplots correspond to abiotic noise from hurricanes Irma and Maria. Upward-pointing arrows point to times where the relative $P A L_{r m s}$ of individual axes shifted due to the array being swept off its mount and onto its side during Hurricane Irma. 

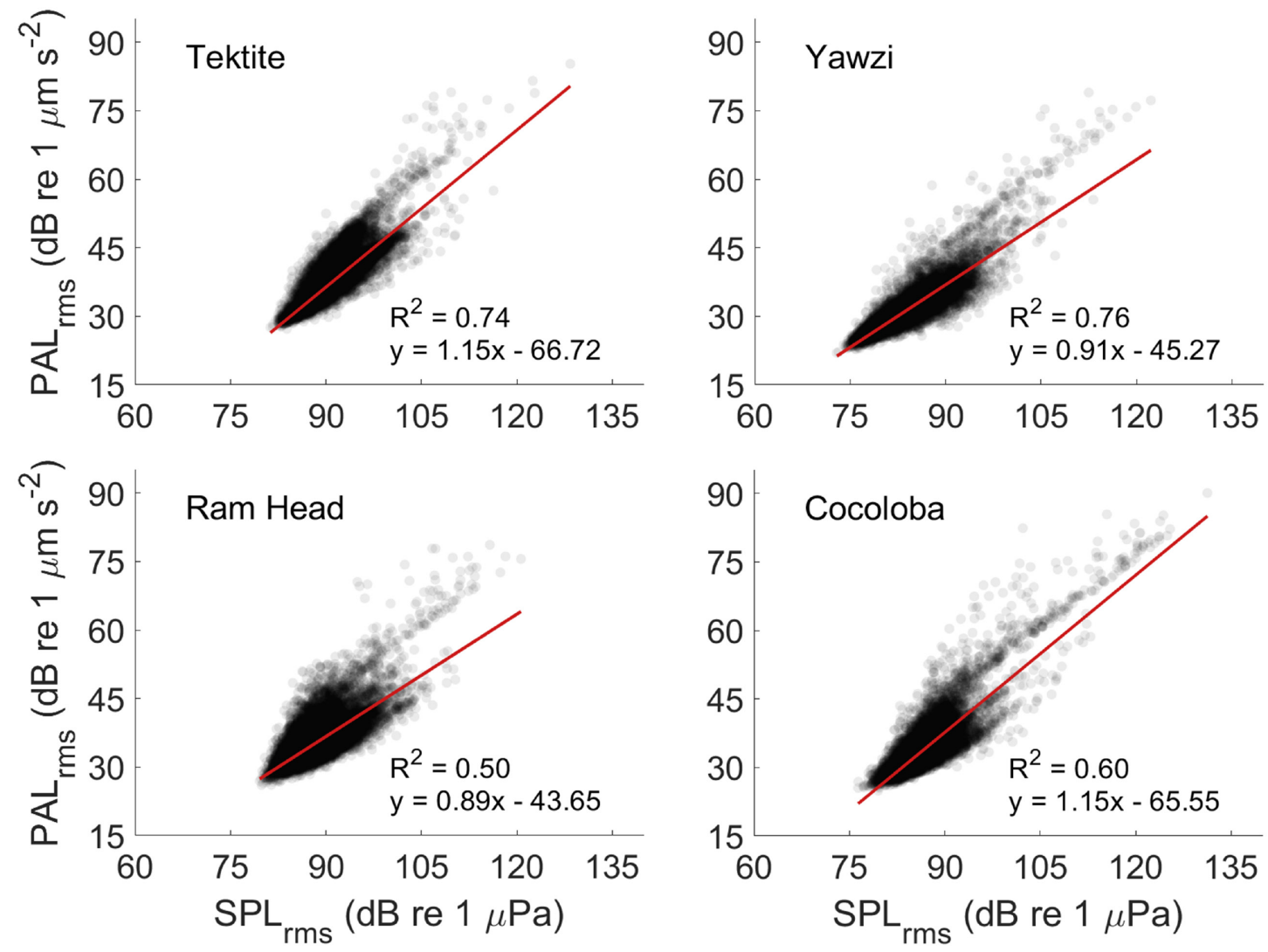

Figure 3. Scatterplots of 3D root-mean-square particle acceleration ( $\left.P A L_{r m s}\right)$ versus sound pressure $\left(S P L_{r m s}\right)$ for $3 \mathrm{~s}$ samples of all recordings from March through August 2017, for each reef site. Linear regression models are shown with red lines and regression equations and Spearman's rho correlation coefficients $\left(R^{2}\right)$ are shown. 

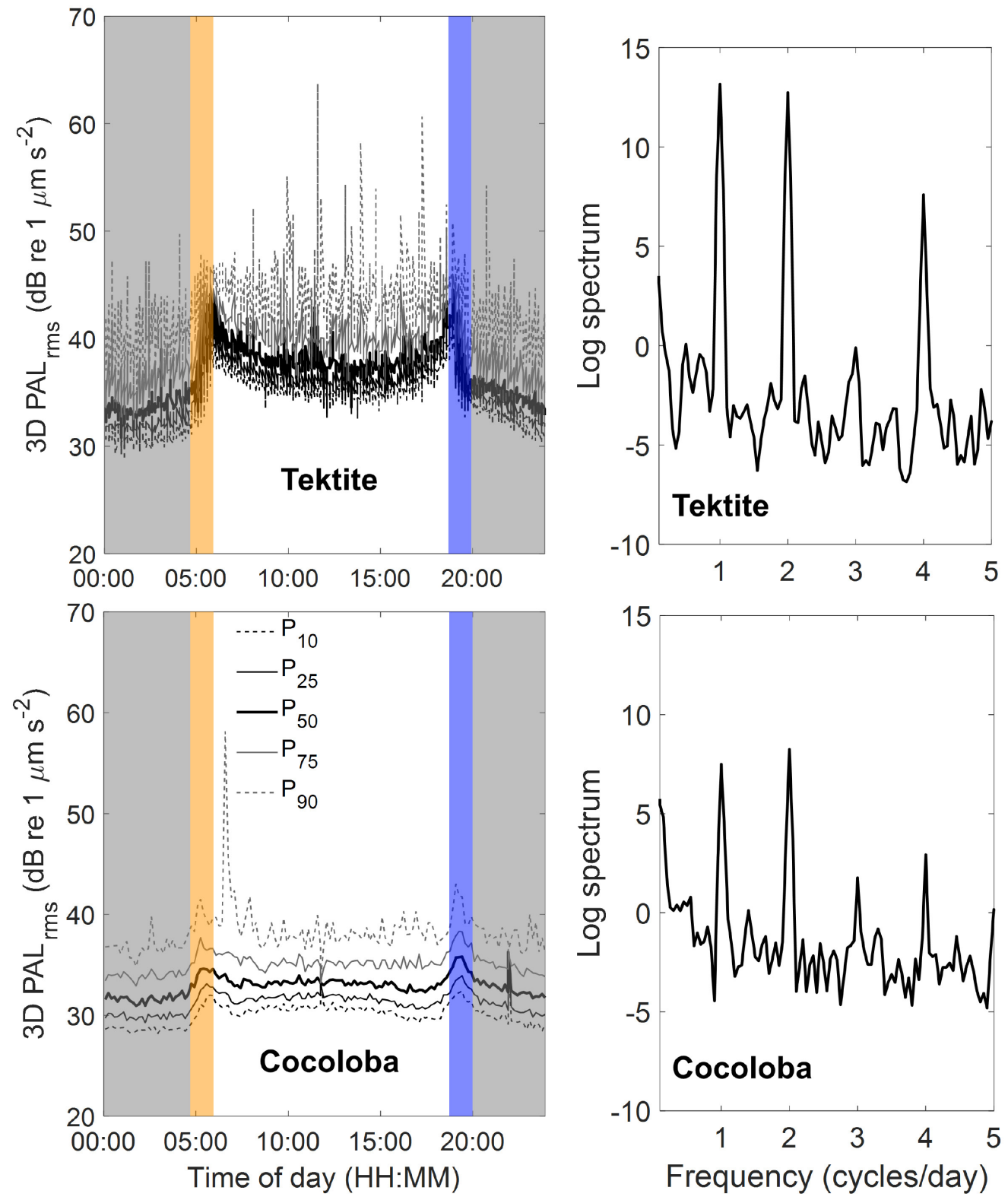

Figure 4. Diel patterns and periodicity of 3D PAL $\mathrm{L}_{\text {rms }}$ for Tektite (top row) and Cocoloba (bottom row) expressed as time-of-day quantiles $\left(10^{\text {th }}, 25^{\text {th }}, 50^{\text {th }}, 75^{\text {th }}\right.$, and $90^{\text {th }}$ percentiles) on the left and periodograms on the right. For quantile plots, white background indicates day, gray background indicates night, and orange and purple bars indicate dawn and dusk respectively. 
a)

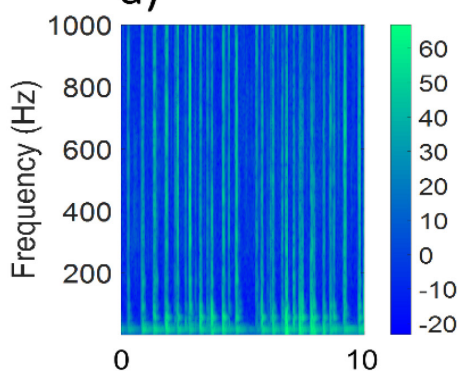

e)
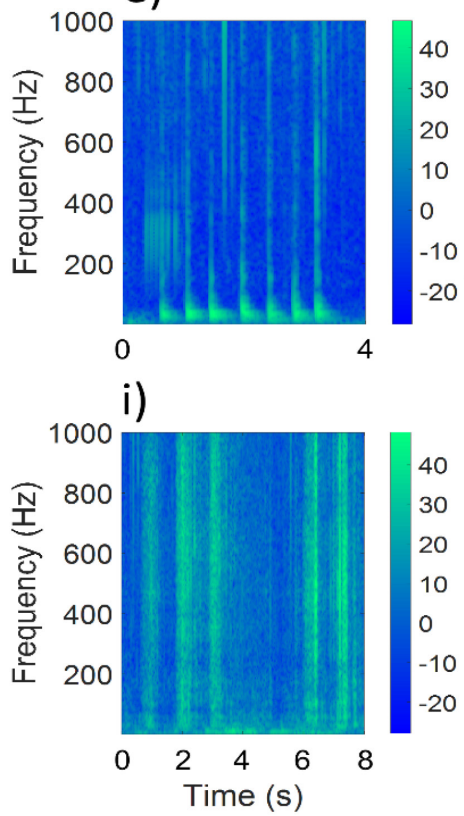

b)

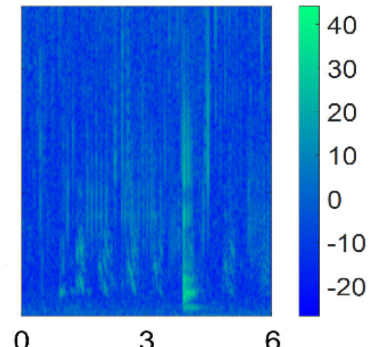

f)

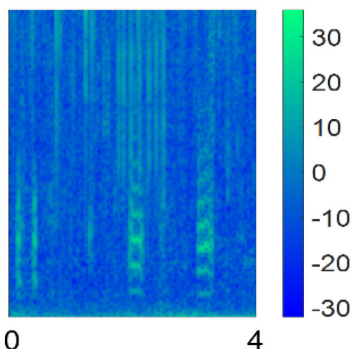

j)
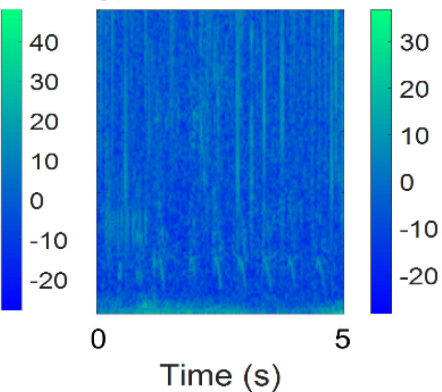

c)

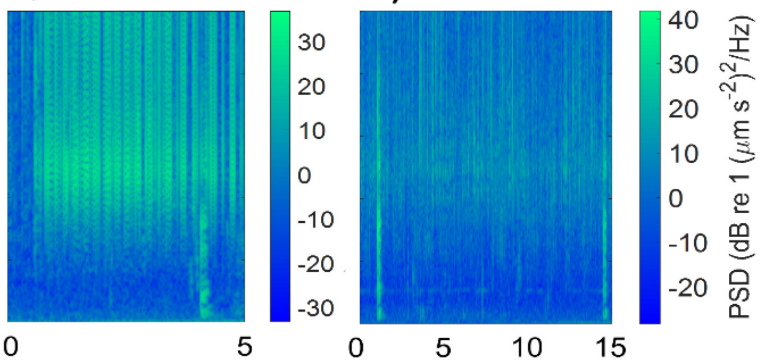

g)

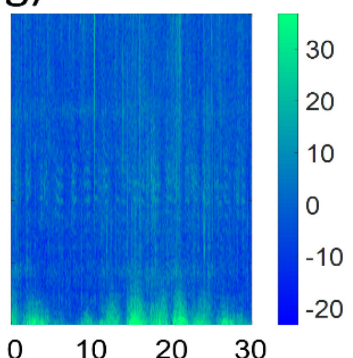

k)

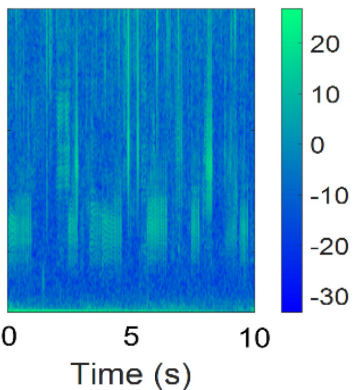

h)

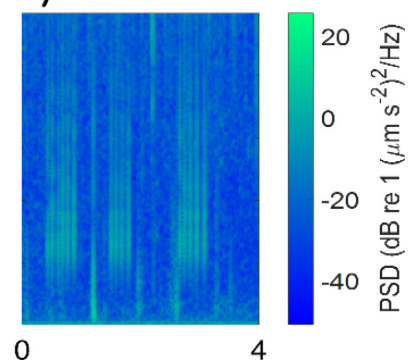

I)

d)

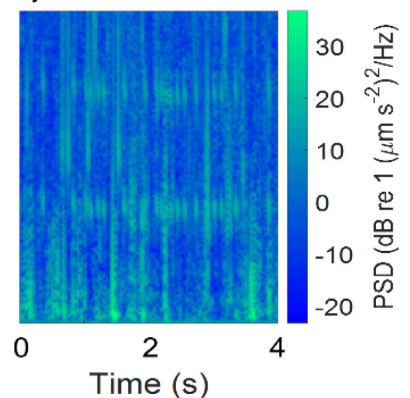

Figure 5. Example spectrograms of fish sounds from files within the $90^{\text {th }}$ percentile of $3 D P A L_{r m s}$ levels at Tektite $(a-d)$ and Cocoloba (e-l). Note that all y axes have the same frequency range but $x$ axes have different time ranges. Colorbars show PSD of 3D particle acceleration. 


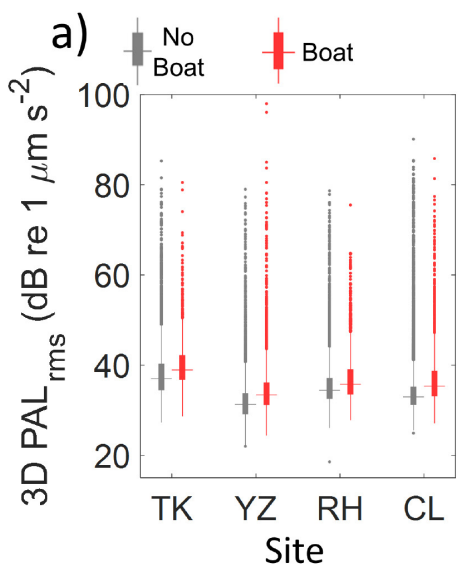

b)

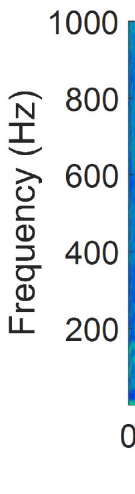

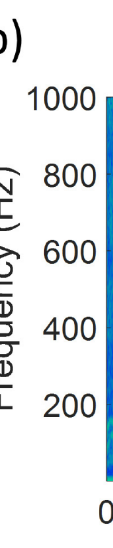

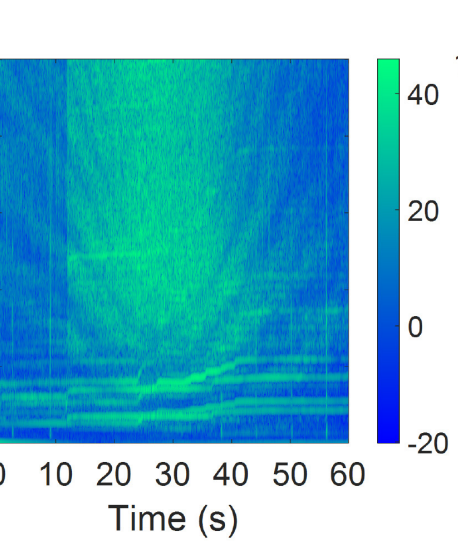

c)

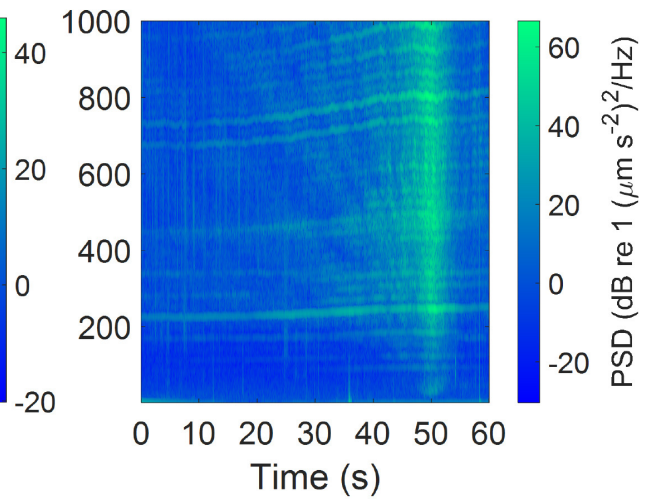

Figure 6. a) Boxplots showing the distribution of 3D PAL $\mathrm{rms}_{\mathrm{rm}}$ for files without boat noise (gray) and files with boat noise (red), for each reef site. Medians are horizontal lines, and boxes extend from the $25^{\text {th }}$ to the $75^{\text {th }}$ percentile. Whiskers extend down to $q_{1}-1.5^{*}\left(q_{3}-q_{1}\right)$ and up to $q_{3}+1.5^{*}\left(q_{3}-q_{1}\right)$, where $q_{1}$ and $\mathrm{q}_{3}$ are the $25^{\text {th }}$ and $75^{\text {th }}$ percentiles, respectively. Outliers are indicated by dots. TK $=$ Tektite, $Y Z=$ Yawzi, $\mathrm{RH}=$ Ram Head, $\mathrm{CL}=$ Cocoloba. b) example boat noise from June $22^{\text {nd }}$ at Tektite. $c$ ) example boat noise from June $16^{\text {th }}$ at Yawzi. Colorbars for $b$ and $c$ show power-spectral-density (PSD) of 3D particle acceleration ( $\mathrm{dB}$ re $\left.\left(1 \mu \mathrm{m} \mathrm{s}^{-2}\right)^{2} \mathrm{~Hz}^{-1}\right)$. Both boat noise examples were within the $99^{\text {th }}$ percentile of 3D particle acceleration levels. 
Figure 7. Peak PSD of 3D particle acceleration from Tektite compared with selected previously published audiograms of a) invertebrates and b) fishes. Note that none of these species inhabit St. John reefs and none of the invertebrates inhabit coral reefs. However, these audiogram data cover much of the amplitude sensitivity range of particle motion-derived audiograms available so far for these taxa. c) Audiograms of pressure-sensitive fishes in b compared with peak PSD of pressure from Tektite. Each point (diamond) represents the peak amplitude and frequency for a $3 \mathrm{~s}$ recording. Superscripts indicate species whose thresholds were measured via neurophysiological methods (AEPs; "a") or with behavior ("b"). Magallana gigas (Pacific oyster; Charifi et al., 2017); Doryteuthis pealeii (longfin squid; Mooney, Samson, et al., 2016); Mytilus edulis (blue mussel; Roberts et al., 2015a); Homarus americanus (American lobster; Jezequel et al., 2021); Pagurus bernhardus (hermit crab; Roberts et al., 2016); Panopeus spp. (mud crab; Hughes et al., 2014); Ovalipes catharus (paddle crab; Radford et al., 2016); Pempheris adspersa (bigeye; Radford et al., 2012); Centropristis striata (black sea bass; Stanley et al., 2020); Sciaena umbra (brown meagre; Wysocki et al., 2009); Micropogonias unduluatus (Atlantic croaker; Horodysky et al., 2008); Chiloscyllium plagiosum (white spotted bamboo shark; Casper \& Mann, 2007). 

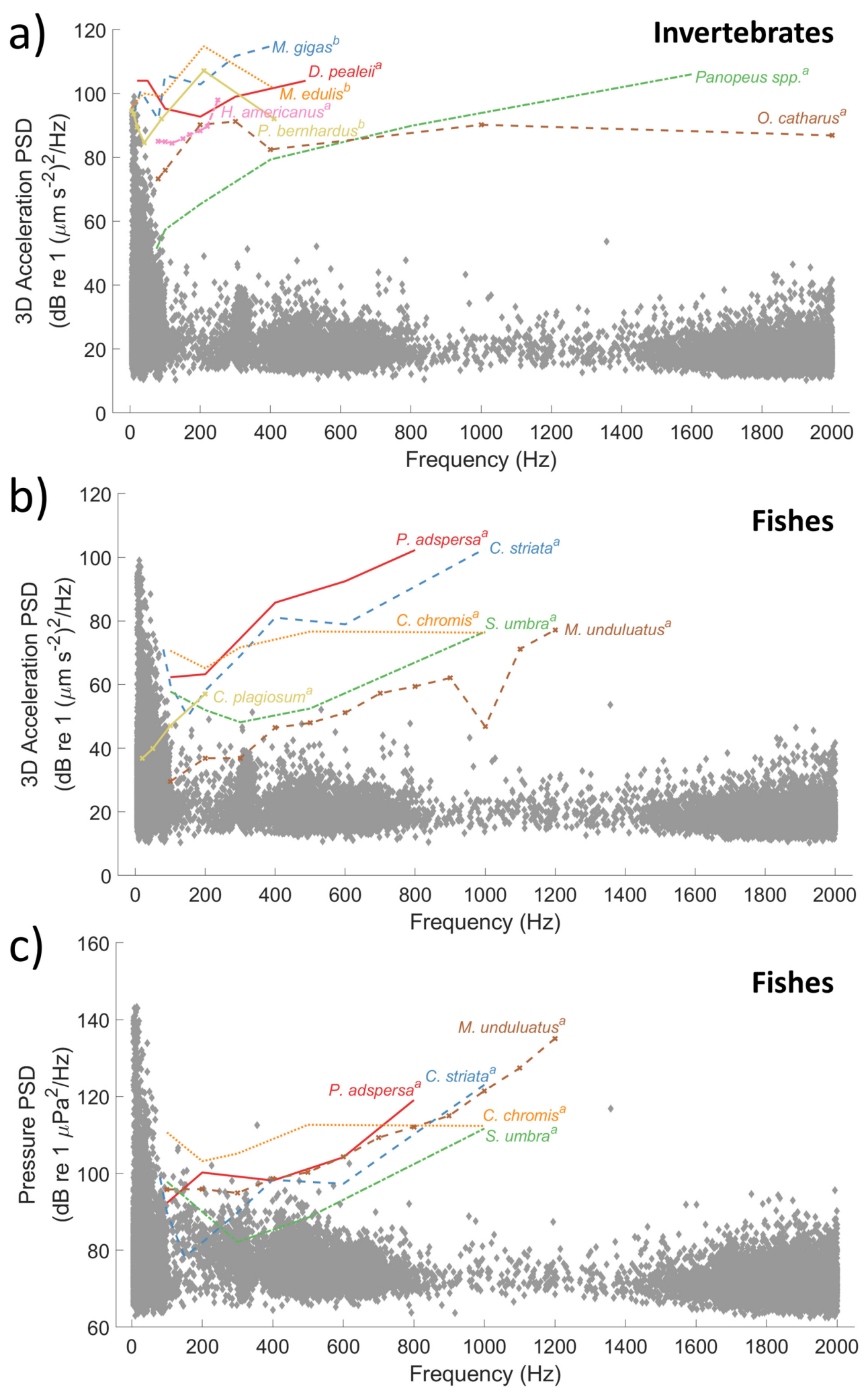


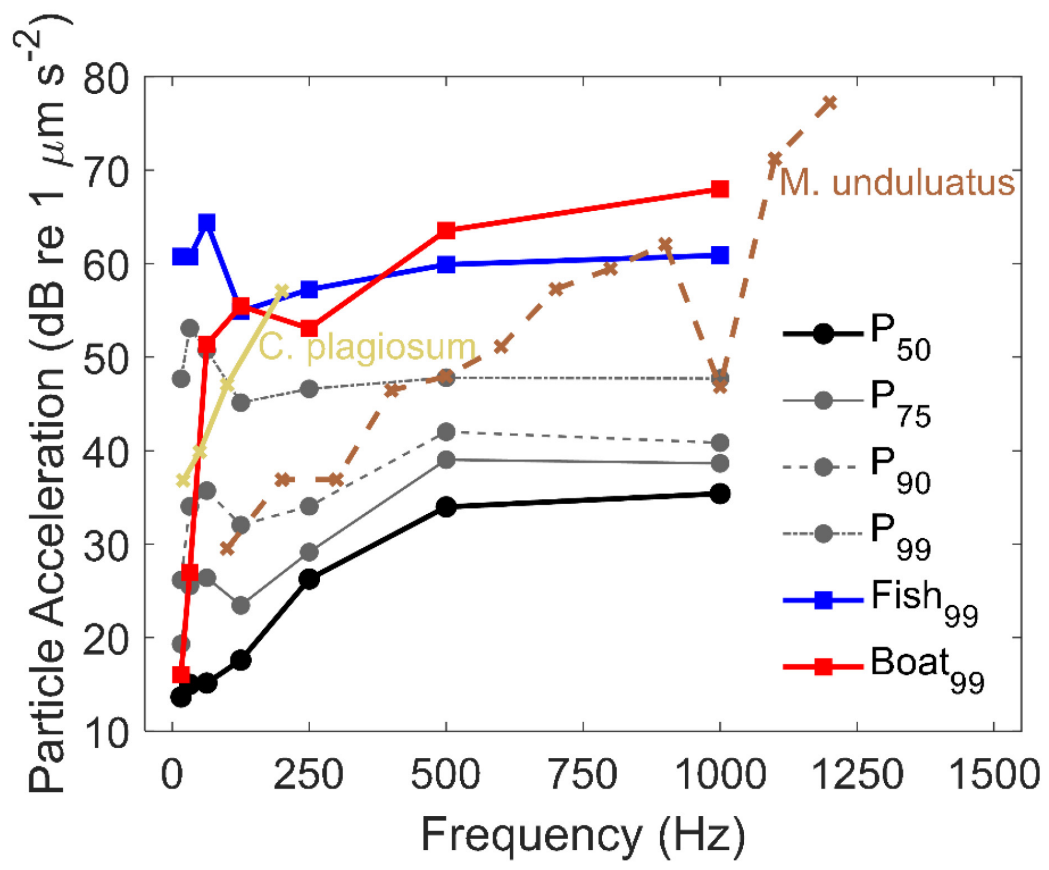

Figure 8. One octave smoothing of 3D particle acceleration PSD data, as percentiles $\left(P_{50}=\right.$ median, $P_{75}=$ $75^{\text {th }}$ percentile, and so on) during dawn at Tektite, across the entire March through August analysis period, (black and gray lines). Also shown are one octave bands levels of an example fish sound (from Fig. 5a, blue line) and boat noise (from Fig. 6b, red line) at Tektite, which were within the $99^{\text {th }}$ percentile of 3D particle acceleration levels. Audiograms are shown of the two fishes in Fig. 7b with the lowest particle acceleration thresholds. Micropogonias unduluatus (Atlantic croaker; Horodysky et al., 2008); Chiloscyllium plagiosum (white spotted bamboo shark; dipole stimulus; Casper \& Mann, 2007). 

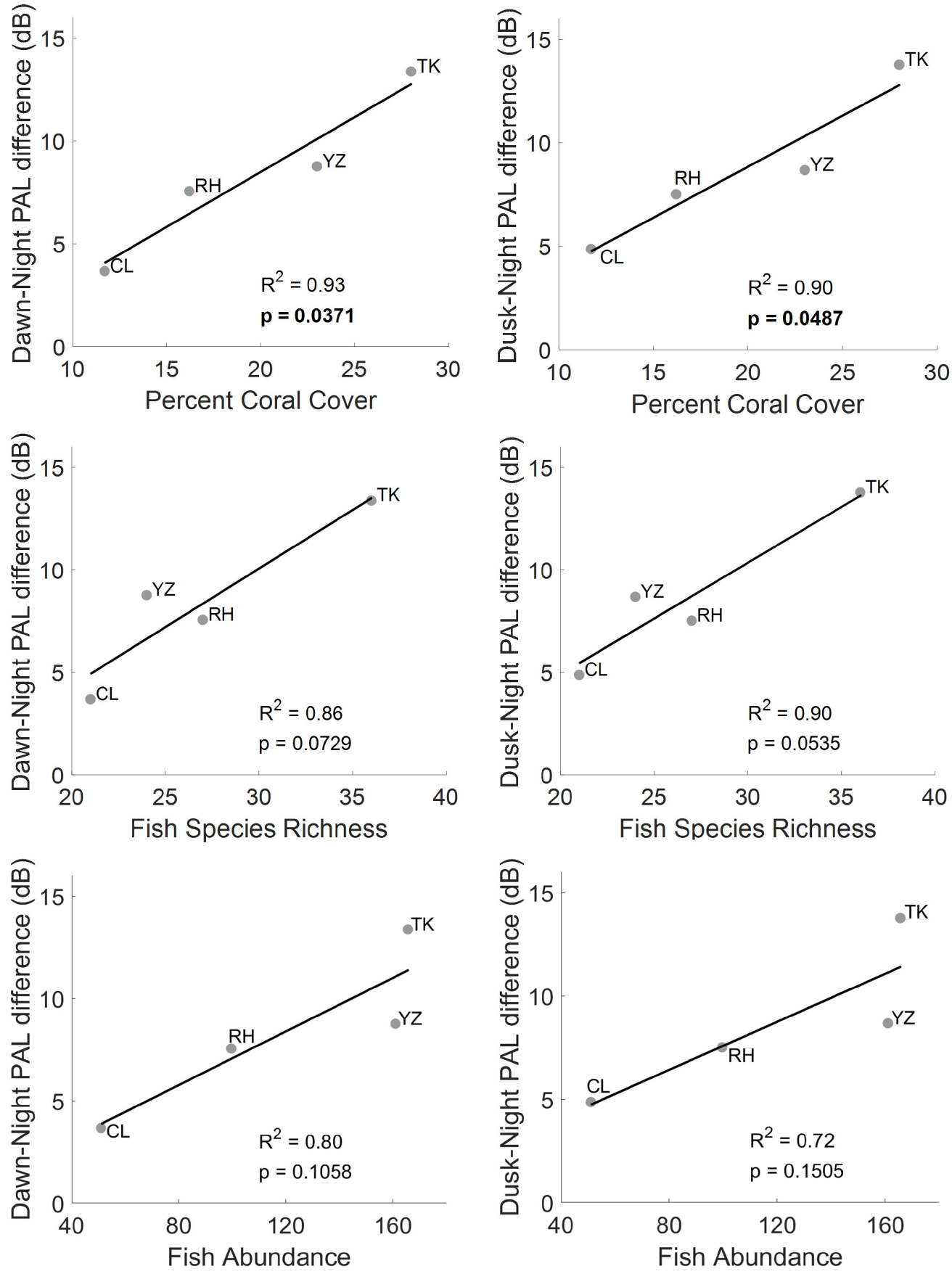

Figure 9. Diel strength of 3D root-mean square particle acceleration ( $P A L_{r m s}$ ) at each site, of dawn peaks relative to night (left column) and dusk peaks relative to night (right column) versus visual survey metrics including percent coral cover, fish species richness, and fish abundance. $R^{2}$ and $p$ values were obtained from Pearson's correlation. Significant $p$ values $(p<0.05)$ are in bold. 
CHAPTER 6 : CONCLUSIONS AND FUTURE DIRECTIONS 


\subsection{A REVIEW OF THE MOTIVATIONS BEHIND THIS THESIS}

Hearing is a vital and basal sensory modality for marine taxa, from invertebrates to fishes and mammals. For all these taxa, natural sound cues serve key ecological functions including navigation, communication, foraging, habitat selection and more. This widespread dependence of marine animals on natural acoustic cues leaves them vulnerable to human perturbations of natural soundscapes. Increasingly pervasive anthropogenic noise in the oceans threatens animals' effective use of natural sound cues, and can elicit a wide variety of adverse effects including physical damage, physiological stress, and behavioral changes. To thoroughly understand sensitivities and responses of fishes and invertebrates to natural and anthropogenic sounds, particle motion must be measured. Additionally, responses to sound should be investigated in multiple ecologically relevant contexts for given species.

Aquatic invertebrates remain far understudied in bioacoustics research relative to fishes and marine mammals, despite their overwhelming contribution to biomass in the oceans, ecological importance in food webs, and their large contributions to global fisheries (Costello et al., 2010; FAO, 2021; Hawkins et al., 2015; Hunsicker et al., 2010). Lack of particle motion data in many bioacoustic studies on invertebrates (and fishes) has impeded progress in this field. For anthropogenic noise and aquatic soundscape studies, lack of empirical, in situ particle motion data has left gaps in understanding of a) what sound levels are present in habitats, b) what sounds invertebrates are able to detect, and $\mathrm{c}$ ) what sound levels they will behaviorally respond to.

Throughout my dissertation I have sought to fill these gaps regarding invertebrate responses to sound and particle motion measurement, with applications to large-scale and time-sensitive management and conservation issues. Construction of offshore wind farms in the U.S. is already underway within squid habitat and fishery grounds (BOEM, 2021; MARCO, 2021). It is critical for stakeholders to have access to empirical data on potential threats these activities pose to wild populations, to allow informed environmental impact assessments and management decisions. Without such data, these construction activities put our resources and the economies of those who depend on those resources (e.g., fisherfolk) at risk. 
Passive acoustic monitoring of natural soundscapes is being increasingly discussed as a tool for assessing ecosystem functions, monitoring habitat health, biodiversity, and impacts of human activities, and even for habitat restoration (Dinh et al., 2018; Elise et al., 2019; Gordon et al., 2019; Kaplan et al., 2015). Among aquatic habitats, coral reefs worldwide are under severe threat from multiple human-driven factors related to climate change, runoff, noise pollution, and more (Altieri et al., 2017; Fabricius, 2005; Ferrier-Pagès et al., 2021; Hughes et al., 2018; Mollica et al., 2018). On top of these stressors, Caribbean reefs have suffered extensive damage from a recent outbreak of a novel, lethal stony coral tissue loss disease (Heres et al., 2021), which by 2020 spread to coral reefs on St. John, USVI, including those studied in Chapter 5 (Roberts, 2020). Threats faced by tropical coral reefs, rapidly changing community structure of reefs (Tsounis \& Edmunds, 2017), and the increasingly recognized ecological functions their soundscapes provide for invertebrates and fishes all make it more timely than ever to investigate coral reef soundscapes. Baseline studies quantifying soundscape levels and spectrotemporal trends will inform efforts to apply soundscape monitoring to protect these habitats. In this concluding chapter, I provide summaries of my major results from each research chapter, then discuss broader ecological and management implications of my findings. Further, I propose foci for future research of anthropogenic noise impacts on cephalopods, and passive acoustic monitoring of underwater soundscapes. Finally, I provide recommendations based on my own experiences these past five years on methodology for particle motion measurement.

\subsection{SUMMARY OF MAJOR FINDINGS}

In Chapter 2, longfin squid responded to pile driving noise with a variety of alarm responses naturally employed for predator defense and evasion. Nearly all squid exposed to noise reacted with at least one of these alarm behaviors, and responses rapidly diminished (squid habituated) within the first minute (first 30 impulses) of noise exposure. A day later, the same squid had resensitized and showed similar response and habituation rates. The type of alarm responses elicited depended on the received magnitude of noise, with inking and jetting 
occurring at higher received particle acceleration levels than other response types. In Chapter 3, effects on feeding behaviors were nuanced: squid that were played noise at the start of their pursuit of prey had significantly more failed predation attempts (missed captures or abandoned pursuits; and similar alarm responses as in Chapter 2), although no significant effects were found on the proportion of squid that ultimately fed. In Chapter 4, there were no significant effects of noise on occurrence rates of any of the reproductive behaviors observed, from fighting behaviors near the beginning of reproductive sequences to egg laying following successful mating. Together these results suggest that antipredator and feeding behaviors may be altered during noise; conversely, squid engaged in reproductive behaviors are highly motivated to continue these behaviors, even during this noise stressor.

Results of Chapter 5 revealed that for many fishes and invertebrates, natural cues with high outlier particle acceleration levels and those at relatively low frequencies are most likely detectable by these animals. Yet, particle acceleration thresholds of many invertebrate and fish species were still above outlier levels. This apparent discrepancy highlights current unknowns and uncertainties in making these comparisons for particle-motion sensitive animals. Particle motion of lower frequency and higher amplitude signals diverged from that predicted by single pressure measurements (using the plane wave approximation). Consistent with sound propagation theory, these results reinforce the conditions for which particle motion can and cannot be reasonably approximated from pressure. Lastly, temporal trends, and relationships between diel cycles and non-acoustic indicators of reef habitat quality were similar when quantified as either pressure or particle acceleration. These findings underscore that when only relative patterns of soundscapes are of interest, such as in long-term monitoring of biodiversity and reef health assessments, reporting of pressure data may suffice.

\subsection{A CONTEXT-DEPENDENT RESPONSES OF SQUID TO NOISE: BROADER ECOLOGICAL AND MANAGEMENT IMPLICATIONS}

Ecological and population-level consequences of anthropogenic noise are inherently difficult to measure. My laboratory-based experiments on squid have allowed identification of several important processes regarding squid responses to noise. These include: response and 
habituation rates, identification of natural behaviors susceptible to change due to noise stressors, and identification of particle motion levels at which they may be expected to occur. These are key initial steps to discerning potential noise impacts. From these findings, one can make educated hypotheses on how, when, and where wild squid may react to pile driving. In the discussion of Chapter 2, I hypothesized that after habituating to noise, squid might similarly become desensitized to predator threats; yet I also point out that research on another squid species revealed habituation specificity to stimuli, i.e., squid that became sensitized to one visual predator model would still respond to a new, different visual predator model (Long et al., 1989). Squid habituation to visual threat cues may or may not carry over to acoustic threat cues. Future experiments modeled after Long et al., (1989) could be conducted to test this. In Chapter 3, I hypothesized that missed opportunities for prey capture and lower feeding rates could lead to reduced growth and survival. Further, considering the metabolic requirements of D. pealeii to feed often, there exists the potential for population level reductions in abundance if wild squid similarly are disrupted from feeding due to a sudden onset of anthropogenic noise. Chapter 4 offers a more positive outlook, indicating squid retain appropriate reproductive behaviors during noise. Therefore, pile driving noise is not expected to reduce the reproductive output of wild populations as far as behaviors up through egg laying are concerned. Potential noise effects on early development have not yet been investigated (see next section). Overall, my results indicate that for $D$. pealeii and perhaps other squid, responses to sound are most likely to occur at the onset of noise, rapid habituation is expected, and reproductive behaviors may be relatively resilient to noise stressors for semelparous species that have limited opportunity to reproduce.

Marine mammal researchers have recently pushed for a more context-based approach toward investigating, monitoring, and managing impacts of anthropogenic noise (Ellison et al., 2012). Similar approaches have not been widely discussed for marine invertebrates, in part due to a paucity of studies investigating effects on a single species in multiple contexts. "Context" can refer to many factors, such as the current state of the animals' environment, physiology, or ontogeny. Here I focus on behavioral context as an overarching theme connected to my research with squid. I propose that similarly for invertebrate taxa, management decisions could 
consider mitigations and development plans around such behavioral contexts and not simply be based on noise amplitudes and frequencies that may generally elicit a "response" (such as an alarm response). For instance, pile driving may not need to avoid times and areas where squid are mating, but considerations could be made to limit exposure at times and in areas many squid are actively feeding. Regulations have been put in place for pausing pile driving based on the presence and activities of nearby marine mammals in some regions, including the United Kingdom and The Netherlands (Bailey et al., 2014; Dahl et al., 2015).

A commonly discussed mitigation method for pile driving is a "ramp-up" procedure, which involves starting pile driving at a lower hammer energy; thus noise impulses are quieter at first, and gradually increasing to a steady operational driving energy. Ramp-up procedures are often used for technical reasons but may serve to "warn" highly mobile animals such as marine mammals, and allow them an opportunity to move further away from the noise source before it reaches louder levels (Bailey et al., 2014; Dähne et al., 2017). Ramp-up benefits make several assumptions including that the animals can, or are motivated to leave an area given the increasing noise stressor, that the cost of leaving is less than that of staying near that stressor, and that animals are making such cost-benefit analyses. Such responses during ramp-up procedures have not been addressed for mobile invertebrates such as cephalopods, or fishes. Measuring directional responses of these taxa to pile driving noise in the field is a high-priority future research objective (see next section). Notably, one study reported trawl survey data of D. pealeii abundance in construction areas ("area of potential effect" or APE) and nearby control ("reference") areas around the Block Island Wind Farm (Carey et al., 2020). This study found reduced squid catches in both APE and reference areas during turbine operation compared to pre-construction baselines. However, catch rate models did not reveal a statistically significant difference and it is unclear if either construction activities or turbine operation influenced squid distribution.

Other mitigation methods can be classified as "engineering solutions" which apply technologies to reduce sounds emissions from pile driving. Such technologies are undoubtedly worth pursuing, but to have an effect on invertebrate (or fish) responses, they must be able to reduce sound emissions at frequencies below $1000 \mathrm{~Hz}$. Bubble curtains immediately 
surrounding the pile can reduce emissions by $10 \mathrm{~dB}$, but are usually more effective at attenuating frequencies above $1000 \mathrm{~Hz}$ (Dähne et al., 2017). Thus they are more relevant to mid- and higher-frequency auditory specialists (e.g., pinnipeds and odontocetes) and would probably be less effective in protecting aquatic invertebrates. Cofferdams (water-tight enclosures) and other various sound dampers and screens placed around piles can also reduce noise emissions by at least $10 \mathrm{~dB}$ (Bellmann, 2014; Bellmann et al., 2020). None of these methods reduce substrate vibrations, which many benthic invertebrates are exposed to, as well as squid egg capsules and adult $D$. pealeii that rest on the substrate. Simply lowering pile hammering energy has the trade-off of requiring longer time to drive piles. To reduce exposure to invertebrates occupying both the water column and substrate, alternative methods of driving that do not release as much acoustic energy to begin with might be more effective. Some alternative driving technologies are being developed that reportedly have lower intensity emissions, such as the "BLUE" hammer which utilizes a constant head of water pressure rather than air pressure or gravity (Koschinski \& Lüdemann, 2020).

\subsection{B FUTURE DIRECTIONS}

My research on squid responses to anthropogenic noise was conducted in wellcontrolled laboratory environments where the noise stimulus, ambient acoustic environment, and physical environment were consistent and known. Yet, animals will behave differently in captivity and sound propagation of pile driving played in a tank is unavoidably different from that in situ from actual pile driving operations. Future comparative experiments should

investigate squid behaviors in field conditions and in the presence of actual pile driving. Ideally, this work should include in situ tracking of squid not confined to enclosures, such as via VHF tags or echosounders. These methods would allow new questions to be addressed, such as whether squid will actively avoid and swim away from noise sources; this question is highly relevant to the fishing industry's concerns about possible reduced catch near wind farms. I recommend such work also focus on antipredator and feeding behaviors, as my studies have revealed significant behavioral changes in these contexts. 
There are a number of additional experiments on squid behavior that would fill some key remaining knowledge gaps, which could occur in controlled tanks or in outdoor enclosures. These include studies I originally proposed for my Thesis but ultimately did not have time for. First, experiments could be conducted to test whether fish predators' feeding behavior on live squid and squids' ability to escape predation are altered during noise. These experiments would help address whether squid alarm responses and subsequent habituation to noise in Chapter 2 would affect their vigilance and responsiveness to actual predator threats. Such studies could be conducted with natural predators of $D$. pealeii including flounder, black sea bass, or bluefish, and be modeled after previous experiments detailing predator-prey interactions among these species (Crook et al., 2014; Staudinger et al., 2011).

Another possible research avenue is to address how noise stressors impact shoaling behaviors of groups of squid. Although alarm responses and feeding behaviors have been studied in solitary squid, D. pealeii and other squid species often shoal (swim together in loosely defined groups) when feeding and migrating (Hanlon \& Messenger, 2018). Multiple studies have been conducted on pile driving and other impulsive noise effects on European seabass shoals, with varying results including increased or decreased group cohesion, and a tendency for fishes to move to deeper water, which together suggest varied anti-predator responses (Herbert-Read et al. 2017; Neo et al. 2015, 2016). Similar studies on groups of squid may be worthwhile, as responses of groups of squid to noise may differ from those of individual squid.

All squid experiments in my Thesis utilized the same pile driving noise playbacks, with playback files being relatively consistent in acoustic properties such as the length of noise impulses and inter-pulse-interval. Yet, several studies on fishes have revealed varied behavioral responses to noise depending on inter-pulse interval, continuous (e.g., white noise or boat noise) versus impulsive (e.g. pile driving, air gun) sounds, and whether sounds have regular or irregular amplitudes and frequencies across time (Blom et al., 2019; Neo et al., 2014, 2015). Similar studies on cephalopods could help predict their responses to a wider variety of anthropogenic noises, including those associated with offshore wind farm development stages other than construction (Mooney, Andersson, et al., 2020). 
Most research conducted thus far on squid or other cephalopod responses to anthropogenic noise has considered adults, and in some cases juveniles. However, pre-juvenile stages of many invertebrates, including cephalopods, have variable sensitivities to environmental stressors that may differ from those of later stages (Zakroff \& Mooney, 2020). Noise has been found to increase mortality, delay development, and cause malformations of sea hare embryos and scallop larvae (de Soto et al., 2013; Nedelec et al., 2014). Although egg laying rates of $D$. pealeii appear unaffected by noise, potential influences of noise exposure on egg hatching and behavior and development of paralarvae (hatched pre-juveniles) have not been investigated.

\subsection{A PARTICLE MOTION OF CORAL REEF SOUNDSCAPES AND THEIR DETECTABILITY BY INVERTEBRATES AND FISHES: BROADER ECOLOGICAL AND MANAGEMENT IMPLICATIONS}

Limited detectability of soundscape cues within reefs, as discussed in Chapter 5, further suggests particle motion of these soundscapes may not be detectable far from a reef, consistent with conclusions of recent studies (Kaplan \& Mooney, 2016; Raick et al., 2021). Even on the reef, only the loudest (or closest) sounds may be detected (Salas et al., 2018). This contrasts with earlier theories that fish larvae could detect particle motion of reef sounds many kilometers away (Radford, Tindle, et al., 2011). Soundscape cues may be more likely to induce settlement of larvae that have already reached reefs, rather than be used for long-distance navigation. Currents and olfactory cues may act to bring larvae to reefs over longer distances (Kaplan \& Mooney, 2016; Paris et al., 2013; Suca et al., 2020). However, we lack particle motion sensitivity data for pre-settlement stage invertebrates and fishes, which may have different sensitivities from adults.

Similar relationships were found between particle motion and pressure, and between each metric and habitat quality data. This indicates that quantifying sound pressure alone may suffice when applying soundscape analyses to monitor biodiversity. Conversely, reporting of particle motion is essential for other management goals. Measuring particle motion will allow more accurate assessments of how boat noise (or other anthropogenic noise sources) will mask 
detection of natural cues by invertebrates and fishes, and what noise intensities are required to alter their behaviors. Basing these assessments only on sound pressure data may lead to erroneous conclusions (Popper \& Hawkins, 2018).

\subsection{B FUTURE DIRECTIONS}

My work highlights significant baseline knowledge gaps regarding how invertebrates and fishes detect and utilize natural soundscape cues. There is much research to be done toward obtaining a more comprehensive understanding of the acoustic ecology of these taxa on coral reefs. Baseline research proposed below will allow more informed applied work utilizing passive soundscape monitoring for conservation and management of underwater habitats. These studies will allow better assessments and predictions of how anthropogenic noise, including that from boat traffic, may impede animals' detection of and responses to ecologically relevant cues.

First, far more research is needed to quantify particle motion detection thresholds of invertebrates and fishes. As discussed in detail in Chapter 5, these should consider responses to ecologically relevant sounds (if known for a given species, for example courtship calls of plainfin midshipman) and not only pure tones. Such studies should also consider using dipole fields which may more realistically reflect sound fields of natural signals. Further, more hearing studies should measure behavioral responses, which are often found at lower thresholds than physiological responses for given species, and examine ontogenetic variation in responses to sound.

Second, more work is needed to identify sound producing species on coral reefs, including Caribbean reefs. This has been done for some reef fishes on Hawai'i (Tricas \& Boyle, 2014). Such work requires co-located visual and acoustic data from divers, as done by Tricas and Boyle (2014), or via deployed, synced camera and acoustic array systems. The latter have been recently deployed at kelp forests off southern California and at reef sites in St. John (C. Pagniello, unpublished data). More comprehensive libraries of sounds produced by fish and 
invertebrate species will aid investigations of ecological functions of these sounds. Ideally, such studies should aim to quantify particle motion levels as well as sound pressure.

\subsection{RECOMMENDATIONS FOR LAB AND FIELD-BASED PARTICLE MOTION MEASUREMENT}

Principles and recommendations for measuring and reporting underwater particle motion have been previously published in several highly useful, but scattered sources (Campbell et al., 2019; Gray et al., 2016; Hubert et al., 2016; Nedelec et al., 2016; Parvulescu, 1964; Rogers et al., 2016). Some user-friendly software for calculating particle motion is available (Nedelec et al., 2016), and suggested standard definitions and units for particle motion metrics are provided by ISO/DIS (2017). Despite these resources, many details about these methods remain esoteric, and progress has been impeded by lack of "official" measurement standards and comprehensive "best practices" guides, which exist only for sound pressure measurements (e.g., Robinson et al., 2014). Though I cannot alone claim what the "best practices" are, I have learned some valuable lessons about particle motion measurement from experience, literature, and personal conversations with researchers experienced in these measurements, that I wish to synthesize here. Based on my dissertation work, I describe here my recommendations for instrumentation and deployment methods for tank-based bioacoustic studies and for passive acoustic monitoring of nearshore soundscapes. Appendix $F$ further provides example MATLAB code on how I calculated particle acceleration metrics from "raw" acoustic data throughout this Thesis. Note that different methods than those described here may be more suitable for other research applications or environments not covered in my Thesis.

For tank-based, aquatic bioacoustic studies with invertebrates (or fishes), I recommend use of triaxial accelerometers. They are often manufactured in small, single units allowing direct measurement along multiple axes simultaneously. As long as accelerometers are well calibrated (which can be done in air with a shaker), there are fewer error sources to account for than when calculating particle motion from a hydrophone array. Considering that particle displacements of most natural sounds are on the order of nanometers (Popper \& Hawkins, 
2018), these transducers must be fixed securely to avoid spurious results from wholetransducer movement. Commercially available accelerometers with waterproof housings are currently lacking. Housings generally need to be custom-made, and the housed unit should be approximately neutrally buoyant, as for the accelerometers I used in Chapters 2-4 (see Acknowledgments). If purchasing just an accelerometer, one will also need signal conditioners, analog filters, and data acquisition boards to obtain raw voltage data to be later converted to acceleration. Unlike many sound pressure recorders (e.g., SoundTraps), accelerometers typically do not come prepackaged with these electronics. Small arrays or pairs of hydrophones are (at present) easier to purchase and construct, and have successfully been utilized to calculate particle motion in several tank-based studies (Mooney et al. 2016; Zeddies et al. 2010). However there are more error sources to worry about: hydrophones used for this purpose should be more carefully calibrated (to a tenth of a dB, ideally), spacing needs to be precise and chosen carefully for the frequency range of interest, and signal to (instrument) noise ratios should be relatively high in order to minimize magnitude errors in calculated particle motion (Gray et al., 2016). I provide further advice from personal experience on measuring particle motion in tanks in Appendix $\mathrm{E}$.

Similarly, for field-based deployments the instrumentation (accelerometer or hydrophone array) chosen to measure particle acceleration may be a matter of availability versus ease of data analysis. A few commercially available particle motion sensors suited for field deployments exist (e.g., from GeoSpectrum Technologies), which were not used in this thesis but were employed in recent field studies (Kaplan \& Mooney, 2016; Mooney, Kaplan, et al., 2016). These have flow-shielded waterproof housings, but have limited compatibility with user-friendly, commercially available recording devices (that set duty cycles, apply anti-aliasing filters, and digitize signals, e.g., SoundTraps). Again, assuming proper calibration and suspension (as recommended by the manufacturer), there are fewer sources of error when using transducers that directly measure particle motion compared to hydrophone arrays. Materials and instruments for constructing hydrophone arrays are more readily available, but the aforementioned error sources must be taken into account, and arrays must be protected from strong bulk fluid flows, if present (Gray et al., 2016). 
Lastly, for bioacoustic studies where invertebrates (or fishes) are concerned, no matter the environment or instrumentation, particle motion and sound pressure should be co-located and measured simultaneously. This allows comparison with the multitude of acoustic studies that only measure sound pressure, and better understanding of relationships between particle motion and pressure, including for noise sources and environments where such relationships are more difficult to predict (e.g., pile driving, in tanks, and near boundaries). Measurement of both pressure and particle motion allows wide application of measurements across animal taxa, invertebrates included.

\subsection{FINAL THOUGHTS}

My dissertation work has pursued multiple, and until recently, rarely studied facets in the realm of aquatic bioacoustics. Magnitudes and propagation of particle motion of many anthropogenic sounds, whether from boats or pile driving, or from natural sounds such as those produced by fishes, are beginning to be quantified more frequently. This will allow improved understanding of the influences of anthropogenic noise pollution and use of natural soundscapes among invertebrates and fishes. My thesis work has contributed to this exciting effort with novel insights on cephalopod responses to anthropogenic noise, and on the availability and potential uses of coral reef particle motion cues for these taxa. My experiments with squid have demonstrated effective use of laboratory tank setups to assess cephalopod sensitivities to anthropogenic noise in multiple behavioral contexts. My experimental setups, noise exposure protocols, and particle motion measurement protocols can be applied to conduct controlled laboratory studies of noise impacts on other species of cephalopods, in other behavioral contexts such as shoaling, and with other types of noise such as boat noise. This research has also laid groundwork for field-based investigations into effects of pile driving noise on squid. I have also contributed novel, baseline particle motion soundscape data that can be leveraged in future investigations of reef inhabitants' production and utilization of these particle motion cues. These data indicate that particle cues that are nearby, high amplitude, and low frequency are most relevant to particle motion-sensitive taxa, and such transient cues 
should be a primary focus of future research on these animals' ecological uses of soundscape cues. More studies measuring particle motion audiograms and standardization of methods to measure them are critical to this effort. Finally, my studies provide observations of interest and use to management entities and stakeholders of offshore wind development, squid fisheries, and those aiming to protect and conserve coral reefs. I anticipate my findings will prove useful toward predicting, monitoring, and mitigating adverse effects of human activities, particularly anthropogenic noise, on natural aquatic resources and habitats. 


\section{APPENDIX A: CHAPTER 2 SUPPLEMENTARY MATERIALS}

This supplement was originally published in: Jones, I. T., Stanley, J. A., Mooney, T. A. (2020). Impulsive pile driving noise elicits alarm responses in squid (Doryteuthis pealeii). Marine Pollution Bulletin, 150. 110792. https://doi.org/10.1016/j.marpolbul.2019.110792. 


\section{Supplementary Results: alarm responses}

Several alarm behaviors occurred at times outside of the first 30 pile impulses (Fig. S1). One squid (in a Day 1 trial) jetted during the last minute of the pre-pile period; this was possibly associated with an accidental door slam that occurred near the experimental area $5 \mathrm{~s}$ prior to this behavior. A different Day 1 squid displayed startle behaviors over four successive impulses along with alternating body pattern changes, flashing from Clear to All dark, then jetted approximately 9.5 min into playback; it was unclear whether this sequence of alarm responses was associated with the squid swimming into the tank wall at this time, and the behaviors were not tightly synced with noise impulses (i.e., they did not occur within $1 \mathrm{~s}$ of impulse peaks). A third Day-1 squid displayed a startle behavior 10.5 min into playback, though it was delayed from the impulse by $>1 \mathrm{~s}$. Two different squid in Day 1 trials displayed one body pattern change before the pile driving noise started, during the acoustic file's $3 \mathrm{~s}$ "fade-in" period. These two squid were likely responding to the increase in background sound level. Three squid (all in Day 2 trials) exhibited alarm-type body pattern changes during the last minute of the pre-exposure period, and 8 squid total in Day 1 and Day 2 trials displayed body pattern changes after 5 min of noise playback elapsed. No control squid displayed alarm-type body pattern changes during the last minute of the pre-exposure period, though two control squid displayed them after 5 min into silent playback: one control squid briefly displayed Bands while swimming (for $<2 \mathrm{~s}$ ) at 04:49 and 11:46 (mm:ss) into silent playback, and the other squid displayed the All dark component when swimming at 13:13 (mm:ss) into silent playback. There were no occurrences of inking, jetting, or startle behaviors in control trials during the last minute of the pre-pile period or during 5-15 min into control playback. 


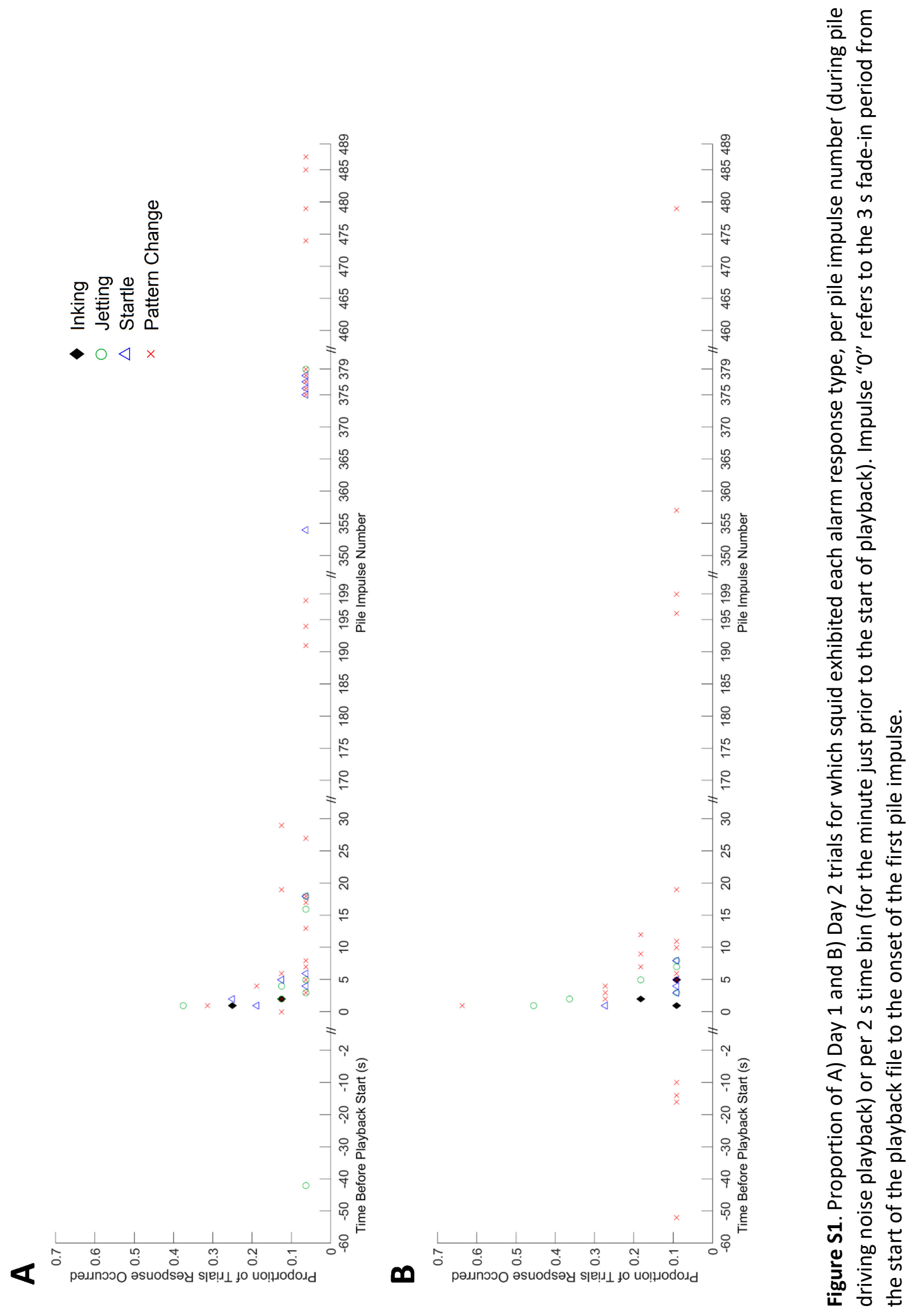




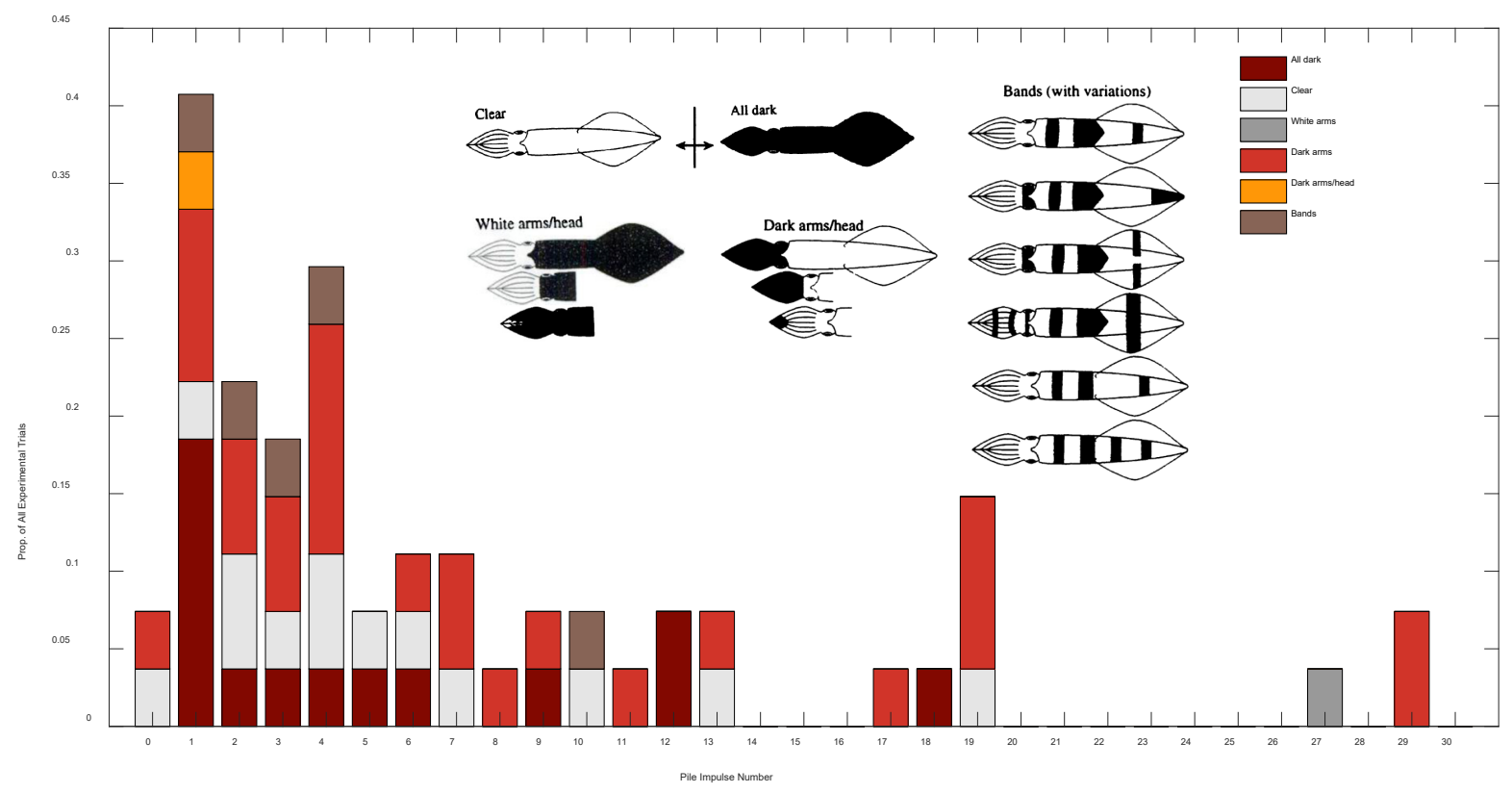

Figure S2. Proportions of all trials (Day 1 and Day 2 combined, excluding controls), for which squid exhibited various chromatic components that make up the body pattern changes reported in Fig 6-7. Proportions are displayed per pile driving impulse for the first 30 impulses. Impulse " 0 " refers to the $3 \mathrm{~s}$ fade-in period from the start of the playback file to the onset of the first pile impulse. Drawings of each chromatic component are shown (adapted from Hanlon et al. 1999). "All dark" and "Clear" chromatic components typically indicate a relatively stronger state of alarm, "White arms", "Dark arms", and "Dark arms/head" typically indicate a relatively milder state of alarm, and "Bands" typically indicates an attempt at crypsis (Hanlon et al. 1999). 


\section{APPENDIX B: CHAPTER 3 SUPPLEMENTARY MATERIALS}

This supplement was originally published in: Jones, I. T., Peyla, J. F., Clark, H., Song, Z., Stanley, J. A., Mooney, T. A. (2021). Changes in feeding behavior of longfin squid (Doryteuthis pealeii) during laboratory exposure to pile driving noise. Marine Environmental Research. 165. https://doi.org/10.1016/j.marenvres.2020.105250. 


\section{Supplementary killifish (Fundulus heteroclitus) Auditory Evoked Potential (AEP) methods}

\section{Killifish placement}

Fish in the AEP recording tank were placed on their left side on a custom holder made with plasticine on top of a plastic board, which was fixed horizontally and at a right angle to a PVC pipe. The fish was wrapped in an elastic restraint that allowed normal respiration through the opercula. The fish was placed with its head about $8 \mathrm{~cm}$ below the water surface and $50 \mathrm{~cm}$ from the front of a horizontally-facing underwater speaker (UW-30) located at the opposite end of the tank, mid-water column, and facing the fish.

\section{Acoustic stimuli}

Fixed-frequency acoustic signals generated by the laptop were converted to analog signals with a National Instruments DAQ (6062E PCMCIA) which was connected to a BNC connector box (National Instruments) then to an attenuator (Hewlett-Packard 350D) that output levels in $5 \mathrm{~dB}$ steps. Signals from the attenuator were wired to an amplifier (PLA-2210, Pyle Audio) then to the underwater speaker. Stimulus duration ranged from 10-30 ms, depending on frequency.

\section{Electrode recordings}

Three 27 gauge, $0.36 \mathrm{~mm}$ diameter, subdermal stainless-steel electrodes (Rochester Electromedical Inc., FL) were inserted to collect AEP signals from the killifish. A recording electrode was placed just dorsal to the operculum, and a reference electrode was placed medially between the nares at the fish's anterior. A ground electrode was placed in the plasticine of the fish holder. Electrodes were connected to a Grass CP-511 bio-amplifier (AstroMed Inc.), which amplified the killifish's neural responses by a factor of 10000 and bandpass filtered them between 10-3000 Hz. A Krohn-Hite 3362 filter (Krohn-Hite Corp.) applied a second bandpass filter between $30-3000 \mathrm{~Hz}$. Electrodes recorded at a $16 \mathrm{kHz}$ sample rate. At least 500 records (of 100 ms duration each, with stimuli presented at alternating phases set $180^{\circ}$ apart) were averaged for each attenuation level tested at a given frequency. 


\section{Signal calibrations in AEP tank}

Sound levels were calibrated at the attenuation levels at which thresholds were found for each fish, using a hydrophone (HTI-96-MIN) attached to a SoundTrap 4300, and a triaxial accelerometer with a custom neutrally buoyant housing (W356B11, PCB Piezotronics Inc.). The accelerometer was not sensitive enough to record stimuli at the amplitudes thresholds were observed at, thus hearing thresholds are only presented in sound pressure, specifically as rootmean-square sound pressure levels (SPLrms). Killifish possess a swim bladder and thus are likely sensitive to sound pressure as well as particle motion (Popper \& Hawkins, 2018). Though it is important to report hearing thresholds in terms of both pressure and particle motion for a thorough understanding of fish hearing (Popper \& Hawkins, 2019), results presented here in sound pressure provide a general indication of the hearing range of this species. 


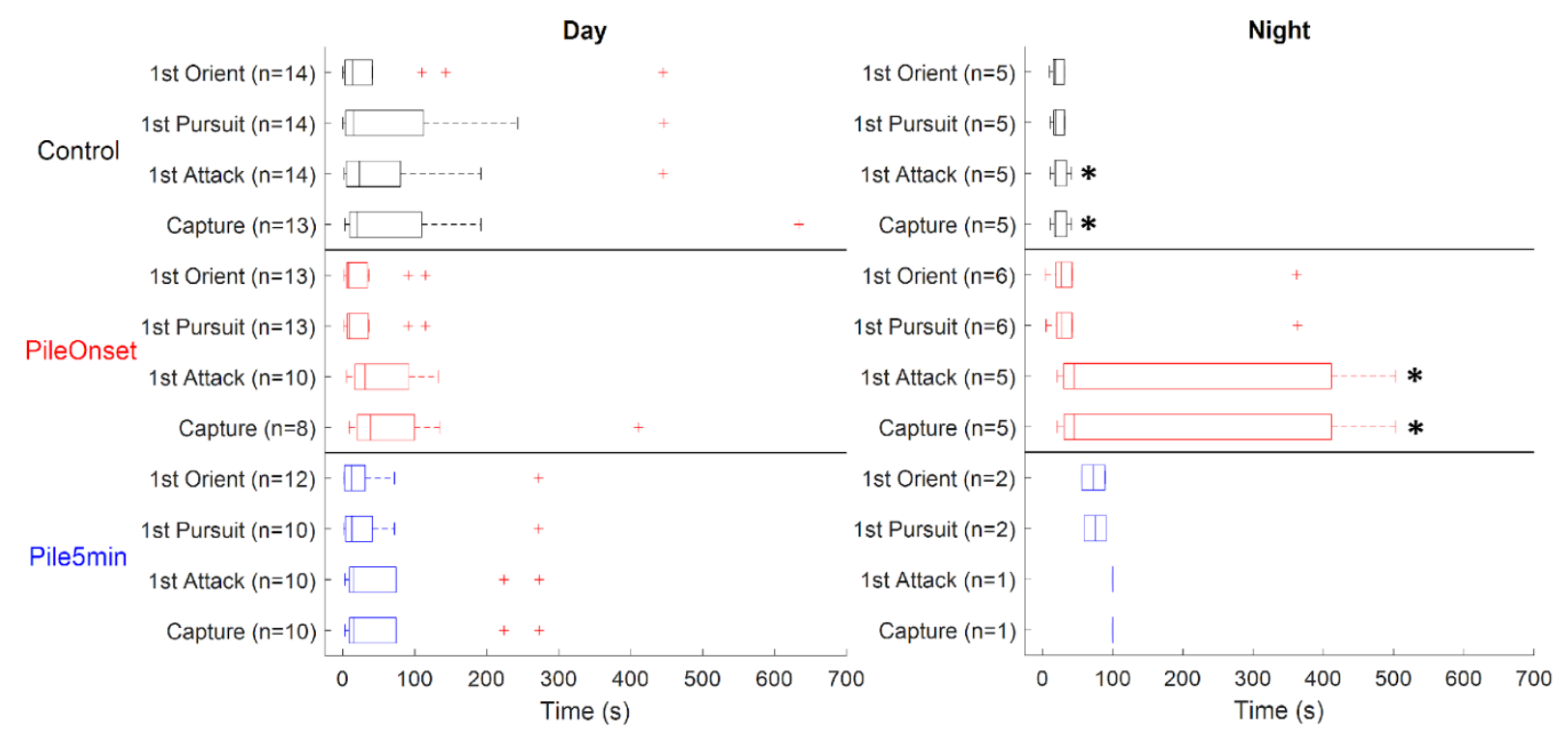

Figure S1. Squid took more time to attack and capture fish only in Night Onset trials. Time from fish reveal to first orient, pursuit, attack, and capture by squid for the three playback treatments, and for Day trials. Outliers (crosses) are defined outside the range $q_{3}+1.5 \times\left(q_{3}-q_{1}\right)$ and $q_{1}-1.5 \times\left(q_{3}-q_{1}\right)$, where $q_{1}$ and $q_{3}$ are $25^{\text {th }}$ and $75^{\text {th }}$ percentiles, respectively. Lines in the middle of boxes indicate medians, and whiskers extend to data points furthest from the median that are not outliers. Only trials in which a silent or pile playback was started are shown. Behaviors shown were analyzed until the end of the playback period. Sample sizes ( $n$ ) presented for individual behaviors are slightly smaller than those presented in Fig. 5 because not all squid exhibited all four possible stages in predation sequences (for example, an attack could occur on a killifish that swam in front of the squid, without a clear orient or pursuit). ${ }^{*} p<0.05$, Mann Whitney $U$ tests. 

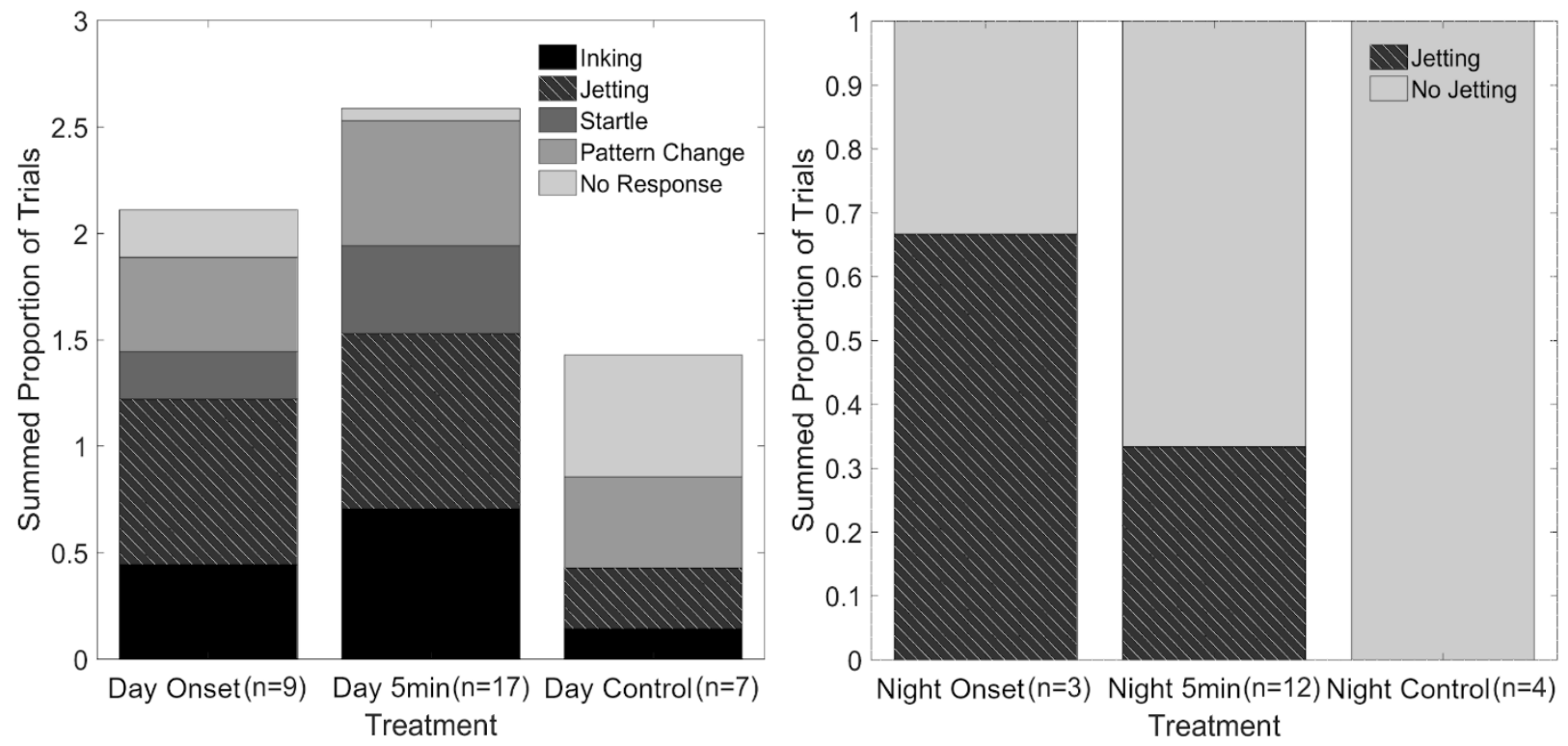

Figure S2. Proportions of each alarm response type were similar between Onset and $5 \mathrm{~min}$ trials, and larger for Onset and $5 \mathrm{~min}$ trials than for Control trials. Stacked bar plot on left shows proportion of Day trials in which inking, jetting, startle behaviors, pattern changes, or no response occurred at least once in the first $60 \mathrm{~s}$ (30 pulses) of playback (proportions were found individually for each behavior category, which, aside from 'No Response', are not mutually exclusive; therefore, summed proportions may exceed 1). Stacked bar plot on right shows proportion of Night trials in which jetting occurred at least once in the first $60 \mathrm{~s}$ (30 pulses) of playback. Only trials for which data was available for 30 pulses are shown here (playback for several Onset and Control trials was stopped before 30 pulses elapsed because the squid had captured the fish). 


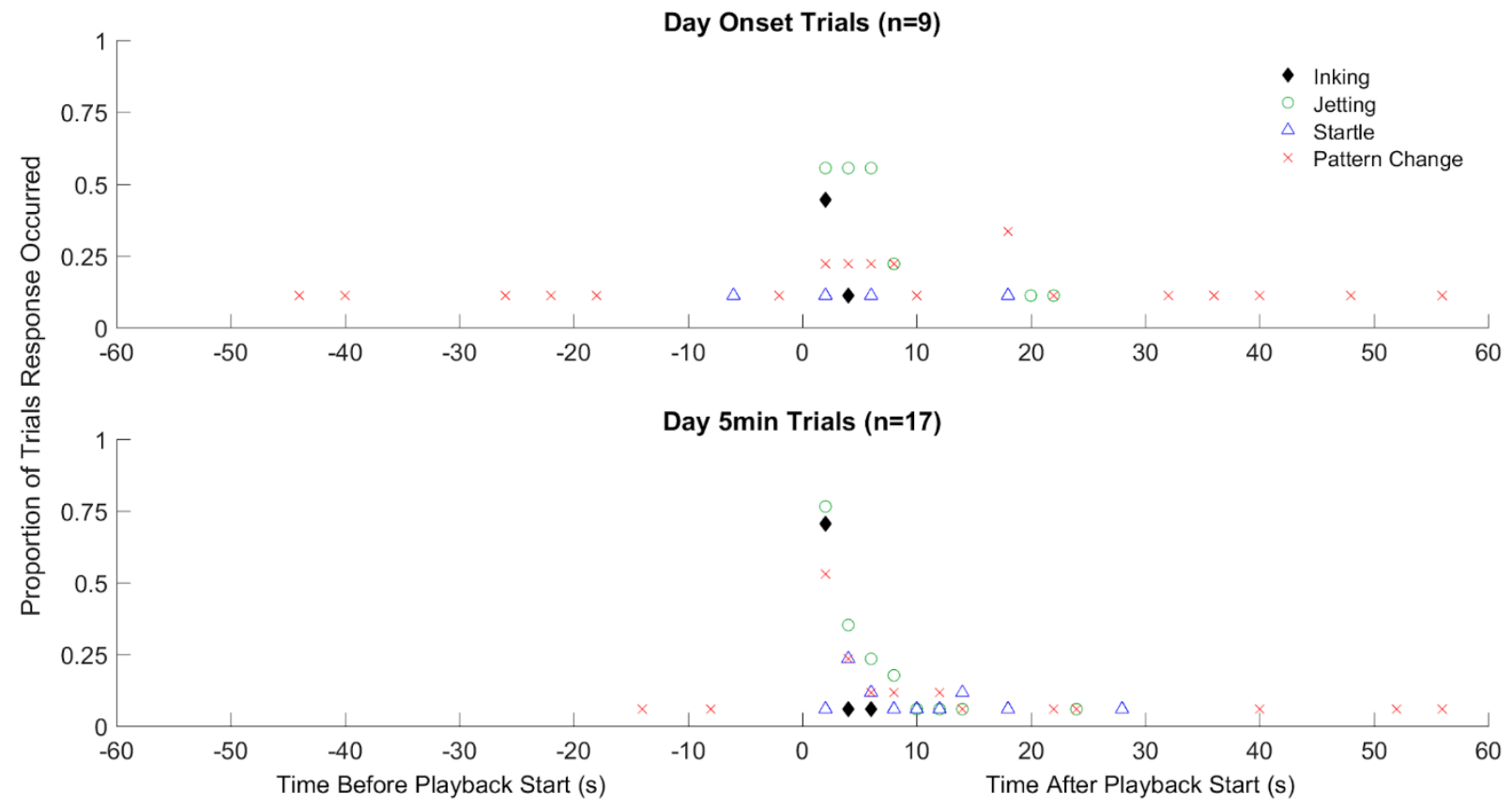

Figure S3. Few alarm responses occurred just before the start of noise playback, and most alarm responses occurred during the first $10 \mathrm{~s}=5$ impulses) of noise playback. Alarm responses at each $2 \mathrm{~s}$ long time bin. Each $2 \mathrm{~s}$ time bin after playback start indicates one pile pulse has elapsed for Onset and 5 min trials (inter-pulse-intervals of $2 \mathrm{~s}$ between impulses). Only trials for which $60 \mathrm{~s}$ (30 impulses) of pile driving were available are included (playback for several Onset and Control trials was stopped before 30 pulses elapsed because the squid had captured the fish). 
Table S1. Detailed statistical results

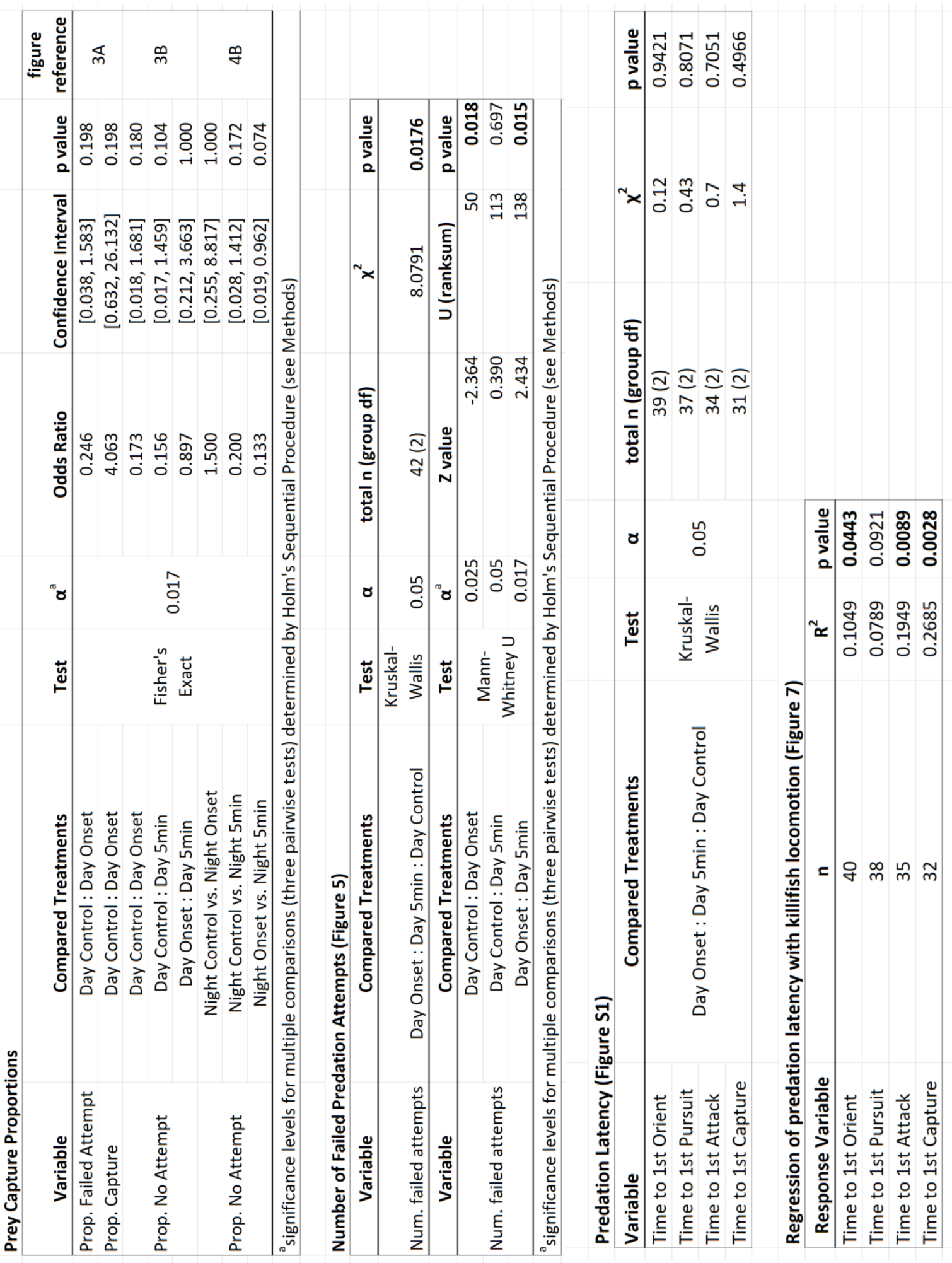



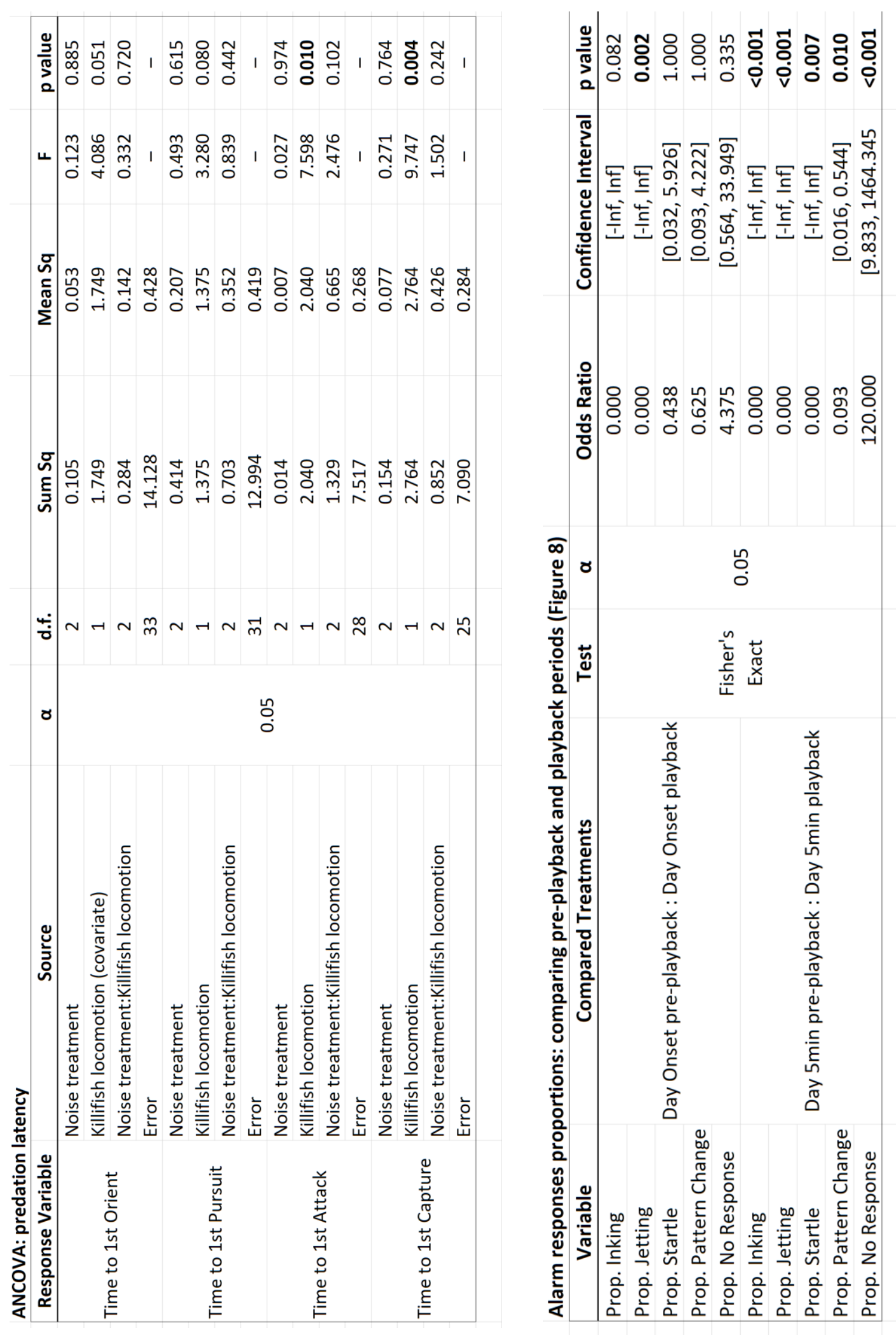


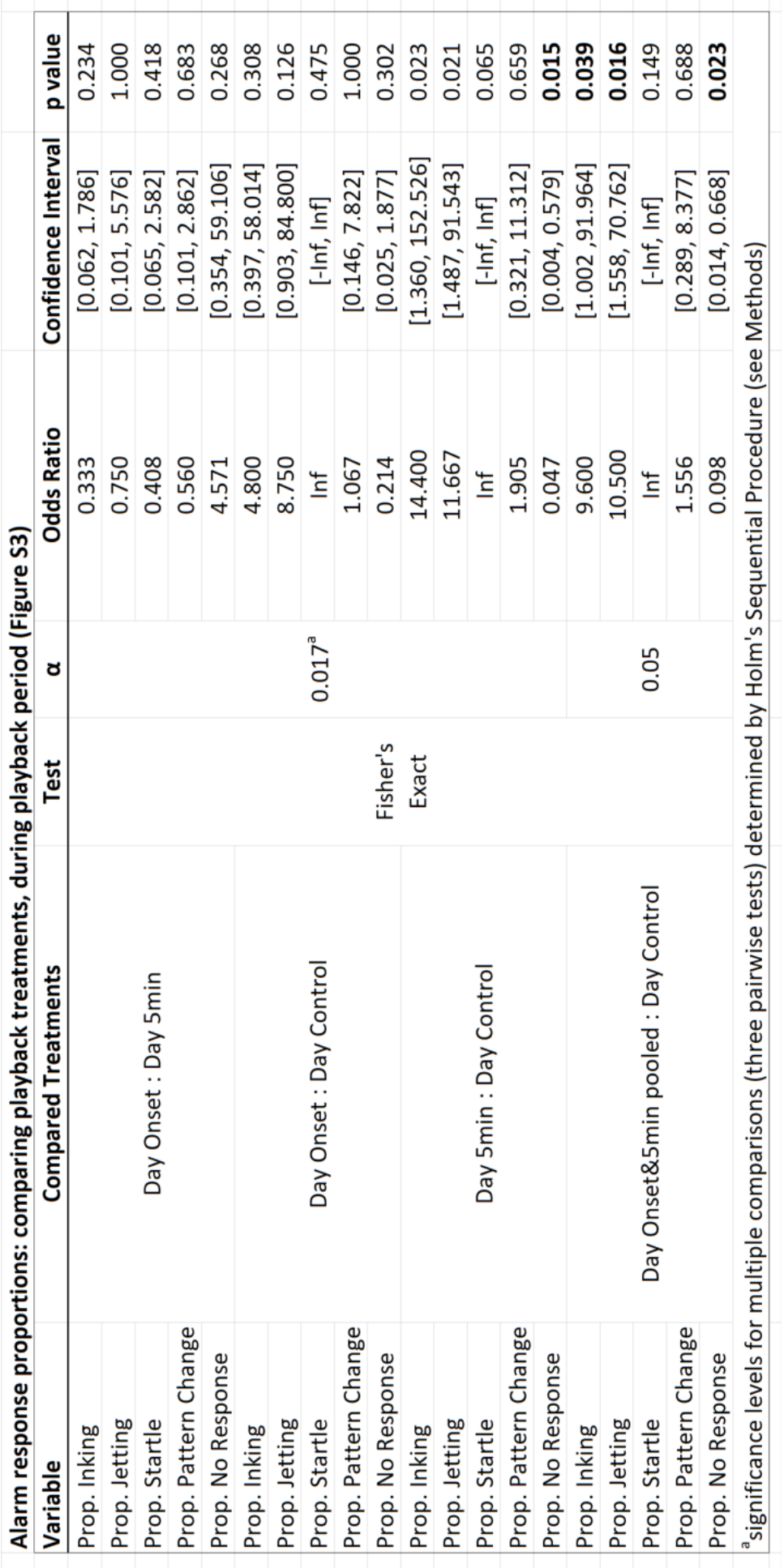




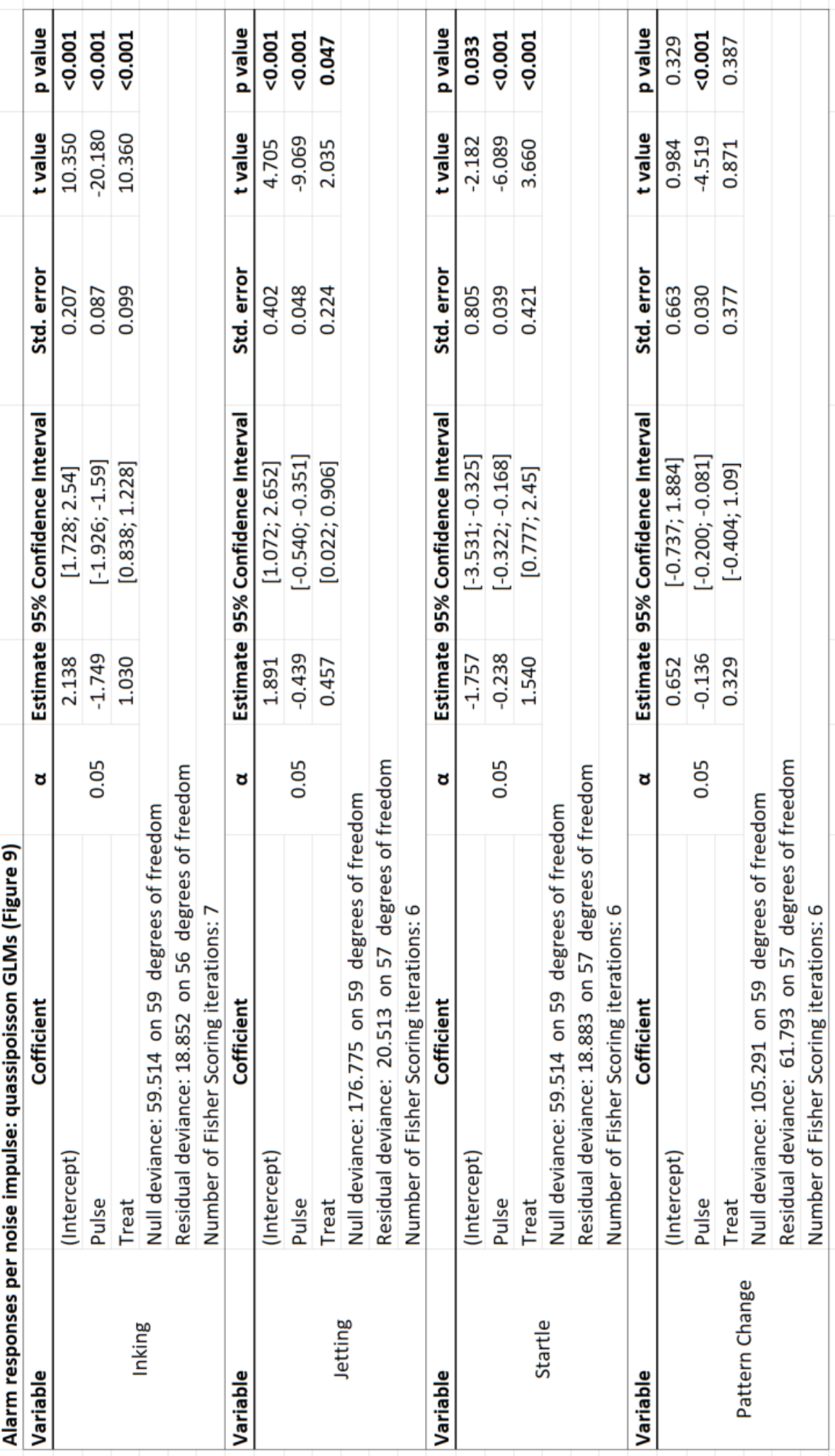


APPENDIX C: CHAPTER 4 SUPPLEMENTARY MATERIALS 


\section{Supplementary Results}

\section{Sound field of experiment tank}

The sound field of pile driving playback in the experiment tank was spatially variable, with highest zero-to-peak levels (up to $145 \mathrm{~dB}$ re $1 \mu \mathrm{m} \mathrm{s}^{-2}$ ) nearest the speaker, and a 20-40 dB drop-off horizontally toward the center of the tank (Fig. S1a). Vertically, sound levels were greater near the center of the tank water column, compared to near the surface and bottom. At all locations, $\mathrm{SAL}_{z-\mathrm{pk}}$ exceeded previously published particle acceleration detection thresholds for D. pealeii (Mooney et al., 2010). A representative recording location was chosen to visualize PSD (amplitude across frequency, Fig. S1b-c). Acceleration levels were about $20 \mathrm{~dB}$ higher in the tank than in the original field recording between $20-100 \mathrm{~Hz}$, and more similar between 400$1000 \mathrm{~Hz}$. Conversely, PSD of sound pressure was lower in the tank than the field recording, across frequencies. Ambient (no playback, speaker unpowered) and silent playback (silent file played through the speaker under power) spectra were similar to each other, and were as much as 50-60 dB lower than the pile driving playback. The accelerometer was not sensitive enough to detect particle acceleration in these two conditions, therefore these recordings resulted in a flat PSD curve at the accelerometer's self-noise floor (55 dB re $1 \mu \mathrm{m} \mathrm{s}^{-2}$, not shown). 


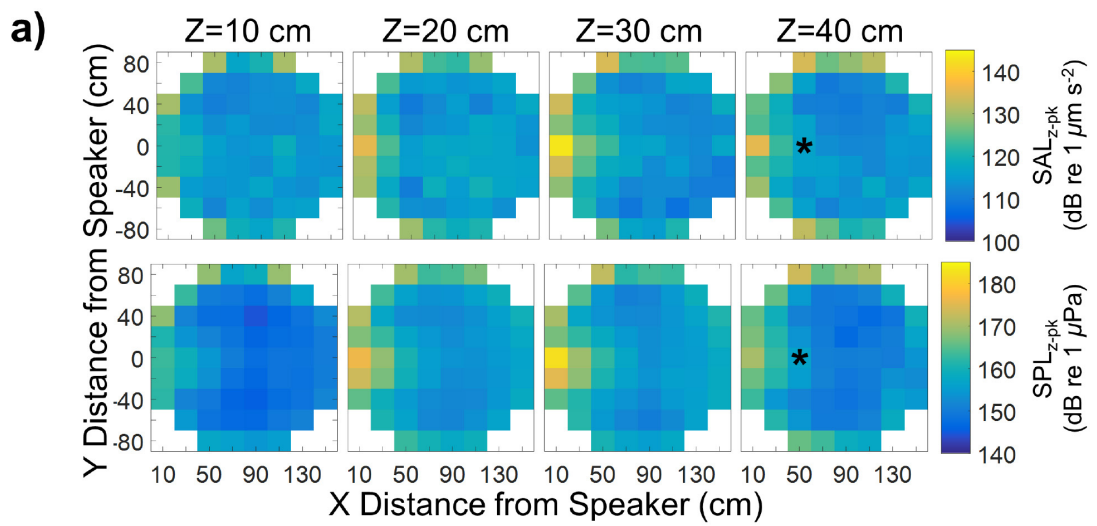

b)

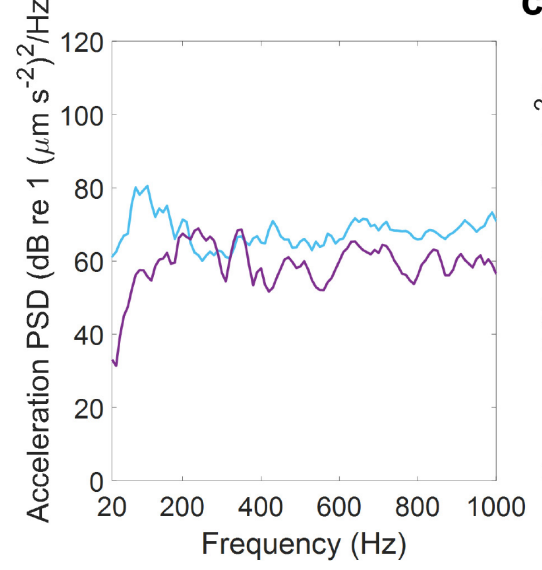

c)

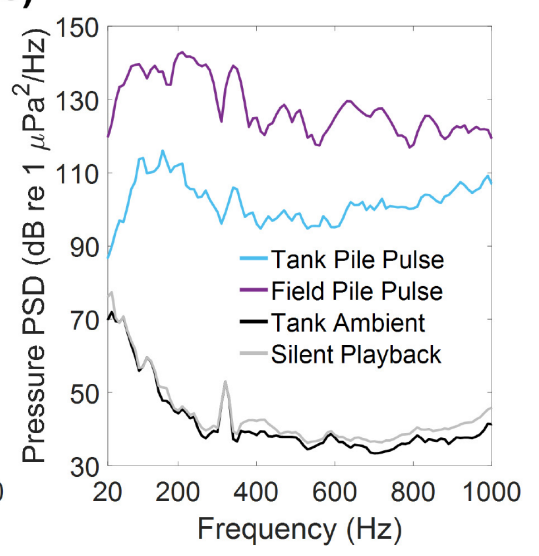

Figure S1. a) Spatial maps of pile driving playback recorded in the experiment tank. The speaker marks the origin of the $X$ and $Y$ axes. Asterisks indicate the location of data shown on the power spectra plots. b) Power spectra of pile driving playbacks in the tank compared with original field recordings, ambient tank sounds, and the silent audio file in the tank. The accelerometer was not sensitive enough to detect ambient and silent playback conditions, hence these spectra are only presented in pressure. 
Table S1. Beta GLMM results comparing mate guarding among time periods, treatment (pile vs. control), for all trials where large males mate guarded. Period $(\mathrm{P})$ and treatment $(\mathrm{T})$ were fixed factors, and squid ID was a random effect ( $\mathrm{rS}$ ), and an interaction between period and treatment (P:T) was included. Significant factors $(p<0.05)$ found in Wald tests are in bold.

\begin{tabular}{lccc} 
Beta GLMM & \multicolumn{3}{l}{} \\
Formula: & Mate guarding $=\mathrm{P}+\mathrm{T}+\mathrm{P}: \mathrm{T}+\mathrm{rS}$ & \\
\hline Coefficient & Estimate & Standard Error & $\mathrm{Z}$ value \\
\hline (Intercept) & -0.20 & 0.49 & -0.41 \\
quiet1 (period) & 0.50 & 0.42 & 1.19 \\
playback2 (period) & 1.68 & 0.49 & 3.44 \\
quiet2 (period) & 2.02 & 0.50 & 4.07 \\
playback 3 (period) & 1.48 & 0.48 & 3.10 \\
treatment & 1.73 & 0.71 & 2.44 \\
quiet1 : treatment & -0.69 & 0.64 & -1.08 \\
pb2 : treatment & -2.04 & 0.69 & -2.94 \\
quiet2 : treatment & -2.23 & 0.70 & -3.20 \\
pb3 : treatment & -1.41 & 0.69 & -2.05 \\
\hline & & & \\
Wald Type II test on beta GLMM & $\mathrm{X}^{2}$ & $\mathrm{df}$ & $\mathrm{P}$ value \\
\hline Coefficient & 10.01 & 4 & $\mathbf{0 . 0 4}$ \\
\hline period & 0.88 & 1 & 0.35 \\
treatment & 13.96 & 4 & $\mathbf{0 . 0 1}$ \\
period : treatment & &
\end{tabular}

Table S2. Beta GLMM results comparing mate guarding among time periods, treatment (pile vs. control), only for trials where large males mate guarded but small males did not. Period $(P)$ and treatment $(T)$ were fixed factors, and squid ID was a random effect ( $r S$ ). Interactive effects were not included because they were insignificant when included in the model. Wald Type II tests did not find any significant factors.

\section{Beta GLMM}

Formula: $\quad$ Mate guarding $=\mathrm{P}+\mathrm{T}+\mathrm{rS}$

\begin{tabular}{lcccc}
\hline Coefficient & Estimate & Standard Error & Z value & P value \\
\hline (Intercept) & 1.50 & 0.53 & 2.85 & $>0.01$ \\
quiet1 (period) & -0.33 & 0.35 & -0.93 & 0.35 \\
playback2 (period) & -0.15 & 0.36 & -0.43 & 0.67 \\
quiet2 (period) & 0.10 & 0.36 & 0.27 & 0.79 \\
playback3 (period) & 0.15 & 0.36 & 0.42 & 0.68 \\
treatment & -0.06 & 0.63 & -0.09 & 0.93 \\
\hline
\end{tabular}

Wald Type II test on beta GLMM

\begin{tabular}{lccc}
\hline Coefficient & $\mathrm{X}^{2}$ & $\mathrm{df}$ & $\mathrm{P}$ value \\
\hline period & 2.69 & 4 & 0.61 \\
treatment & 0.01 & 1 & 0.93 \\
\hline
\end{tabular}


Table S3. Negative binomial GLMM results comparing number of chases by large males among time periods, treatment (pile vs. control), only all trials where large males mate guarded. Period $(P)$ and treatment $(T)$ were fixed factors, and squid ID was a random effect ( $r S$ ). Interactive effects were not included because they were insignificant when included in the model. Wald Type II tests did not find any significant factors.

Negative binomial GLMM

\begin{tabular}{lcccc} 
Formula: & Number of chases $=\mathrm{P}+\mathrm{T}+\mathrm{rS}$ & & \\
\hline Coefficient & Estimate & Standard Error & Z value & $\mathrm{P}$ value \\
\hline (Intercept) & 1.40 & 0.36 & 3.88 & $>0.001$ \\
quiet1 (period) & 0.06 & 0.24 & 0.25 & 0.80 \\
playback2 (period) & -0.17 & 0.26 & -0.67 & 0.51 \\
quiet2 (period) & -0.09 & 0.25 & -0.35 & 0.72 \\
playback3 (period) & -0.18 & 0.26 & -0.71 & 0.48 \\
treatment & 0.58 & 0.42 & 1.37 & 0.17 \\
\hline
\end{tabular}

Wald Type II test on negative binomial GLMM

\begin{tabular}{lccc}
\hline Coefficient & $\mathrm{X}^{2}$ & $\mathrm{df}$ & $\mathrm{P}$ value \\
\hline period & 1.43 & 4 & 0.84 \\
treatment & 1.88 & 1 & 0.17 \\
\hline
\end{tabular}

Table S4. Negative binomial GLMM results comparing number of lunges by large males among time periods, treatment (pile vs. control), only all trials where large males mate guarded. Period $(P)$ and treatment $(T)$ were fixed factors, and squid ID was a random effect $(r S)$. Interactive effects were not included because they were insignificant when included in the model. Wald Type II tests did not find any significant factors.

Negative binomial GLMM

\begin{tabular}{lcccc} 
Formula: & Number of lunges $=\mathrm{P}+\mathrm{T}+\mathrm{rS}$ & \\
\hline Coefficient & Estimate & Standard Error & Z value & $\mathrm{P}$ value \\
\hline (Intercept) & 1.45 & 0.34 & 4.27 & $>0.001$ \\
quiet1 (period) & 0.08 & 0.20 & 0.40 & 0.69 \\
playback2 (period) & 0.16 & 0.20 & 0.77 & 0.44 \\
quiet2 (period) & 0.16 & 0.20 & 0.77 & 0.44 \\
playback3 (period) & 0.02 & 0.21 & 0.11 & 0.91 \\
treatment & 0.18 & 0.43 & 0.43 & 0.67 \\
\hline
\end{tabular}

Wald Type II test on negative binomial GLMM

\begin{tabular}{lccc}
\hline Coefficient & $\mathrm{X}^{2}$ & $\mathrm{df}$ & $\mathrm{P}$ value \\
\hline period & 1.05 & 4 & 0.90 \\
treatment & 0.19 & 1 & 0.67 \\
\hline
\end{tabular}


APPENDIX D: CHAPTER 5 SUPPLEMENTARY RESULTS 


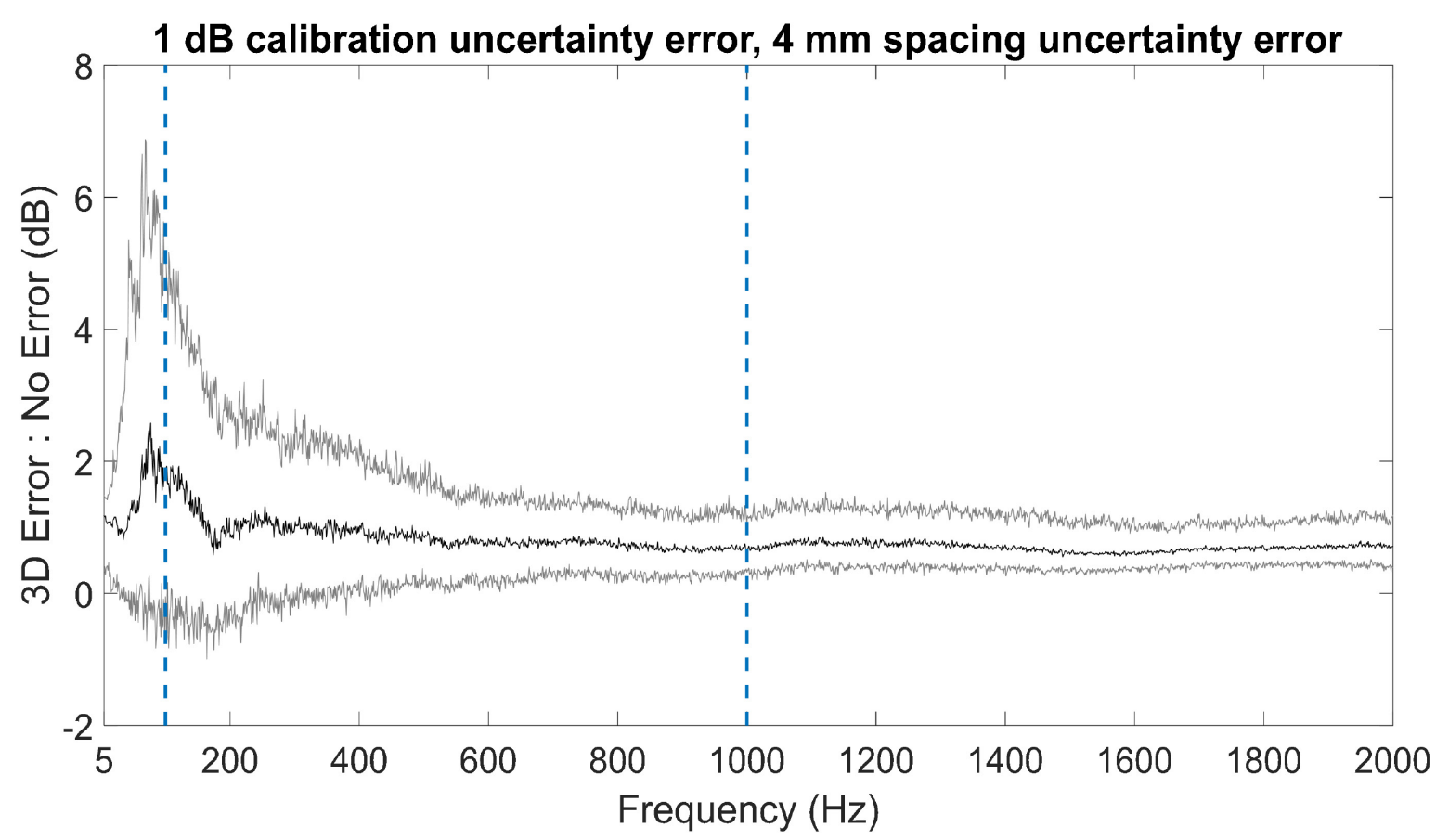

Figure S1. Sensitivity analysis of amplitude error calculated from the finite difference approximation, with a minus $1 \mathrm{~dB}$ error in the calibrated sensitivity for the center hydrophone (hydrophone 4 ) and minus $4 \mathrm{~mm}$ error in hydrophone spacing ( $34 \mathrm{~mm}$ instead of $38 \mathrm{~mm}$ ). These errors are within ranges expected based on hydrophone manufacturer specifications (High Tech Inc., USA) and for realistic hydrophone array setups (Gray et al., 2016). This analysis was done with 100 randomly sampled files from Cocoloba. Power spectral density (PSD) was calculated for 3-s long samples as described in Chapter 5 Methods. The $Y$ axis shows the ratio of 3D particle acceleration PSD with error applied to that without error applied. This ratio was taken for each file, and the median of these ratios is shown in the middle black line, with $5^{\text {th }}$ and $95^{\text {th }}$ percentiles above and below in gray. Vertical dashed lines indicate the $-3 \mathrm{~dB}$ cutoffs (100 and $1000 \mathrm{~Hz}$ ) for the frequency band over which root-mean-square levels were calculated. 

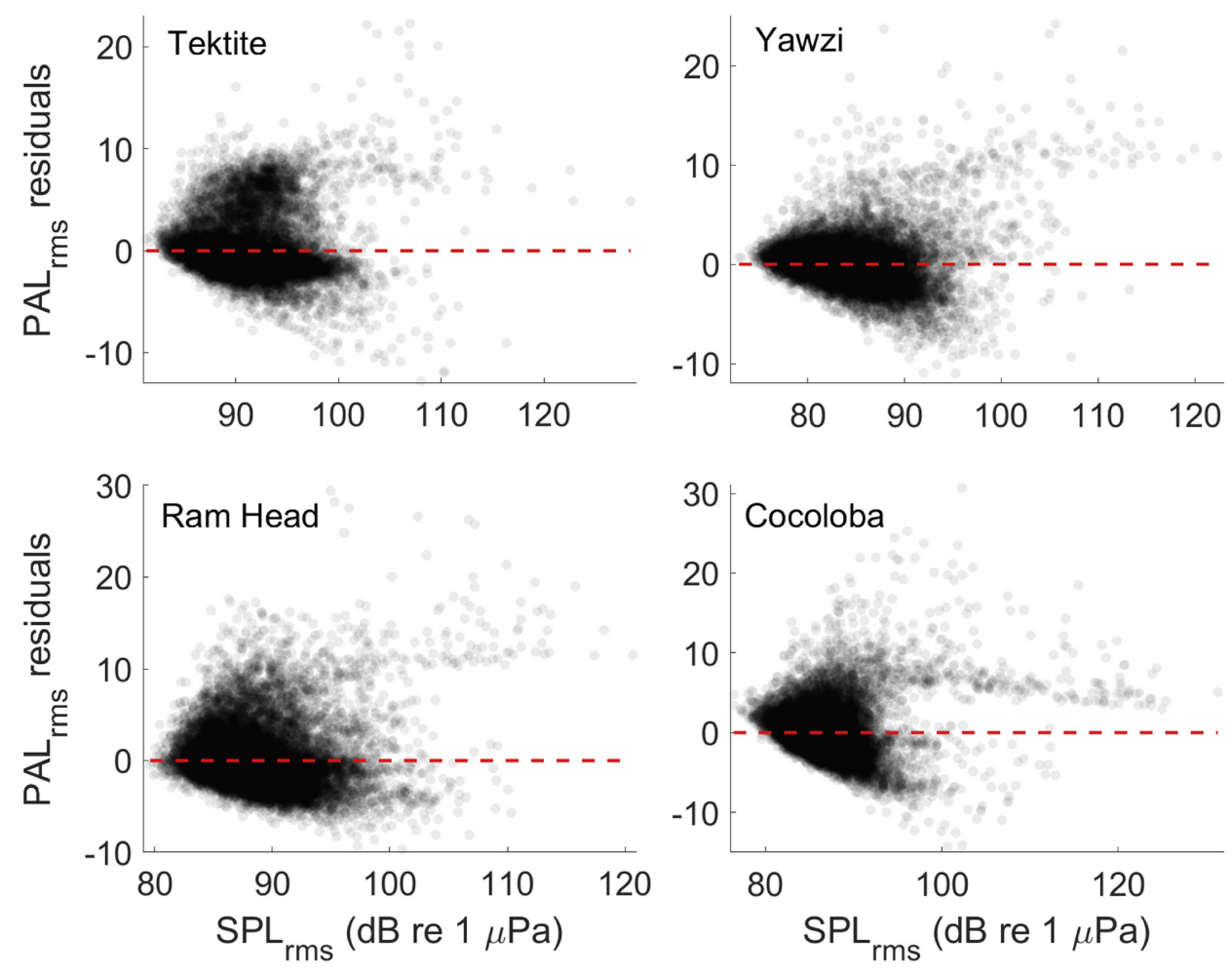

Figure S2. Residual plots for the regression analysis shown in Fig. 3. PAL rms $_{\text {r }}$ residuals are of particle acceleration data in units of $\mathrm{dB}$ re $1 \mu \mathrm{m} \mathrm{s}^{-2}$. Red dashed lines indicate the linear regression line. 
finite-difference

\section{Tektite}

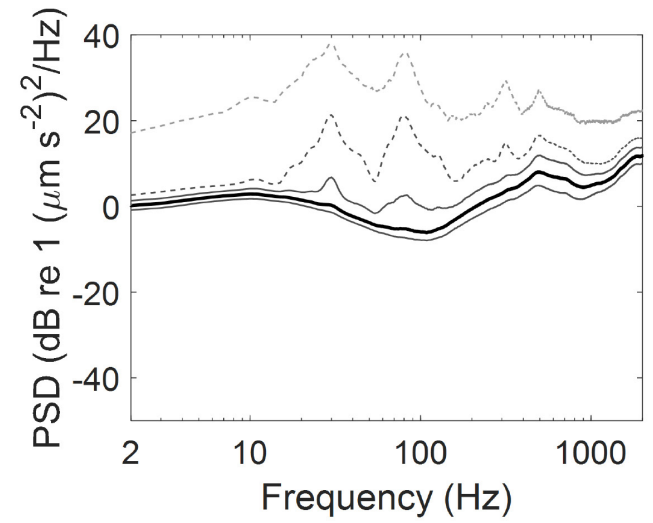

\section{Ram Head}

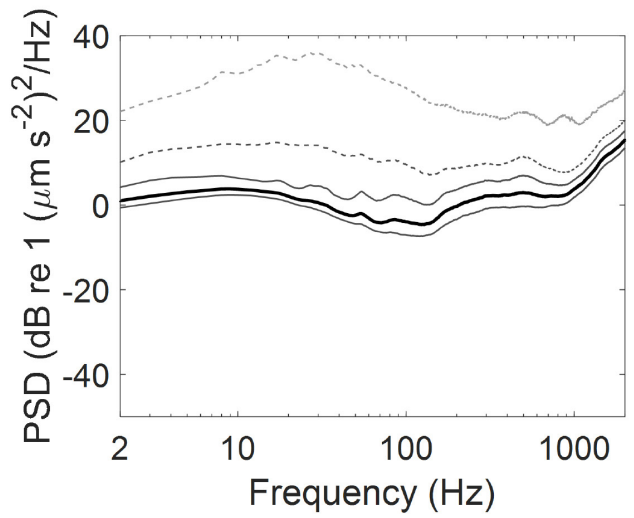

plane wave approximation

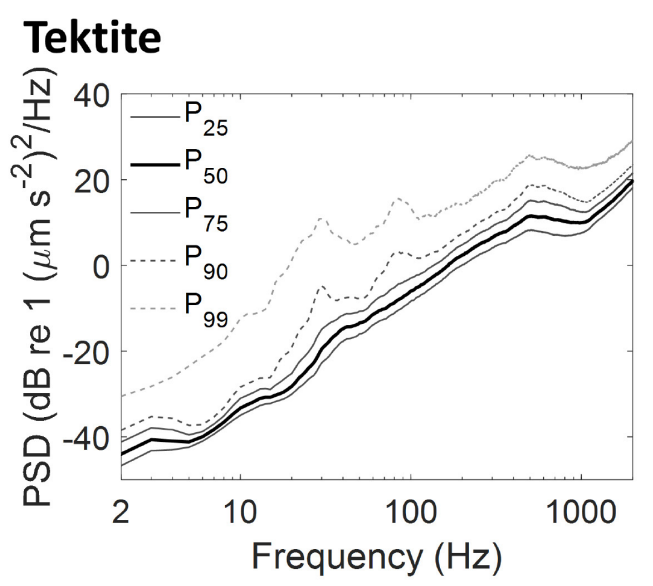

\section{Ram Head}

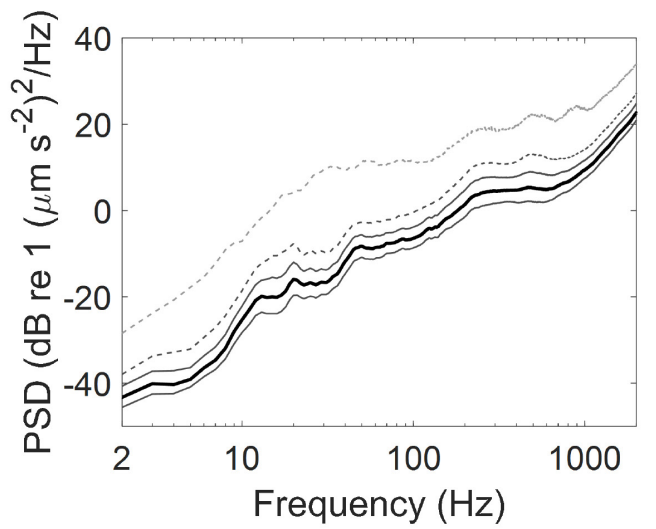

Figure S3. Power spectral density of particle acceleration at $25^{\text {th }}, 50^{\text {th }}, 75^{\text {th }}, 90^{\text {th }}$, and $99^{\text {th }}$ quantiles, as obtained via the finite-difference approximation (left; Equation 5), and the plane wave approximation (right; Equation 7, shown for Tektite (top) and Ram head (bottom). 

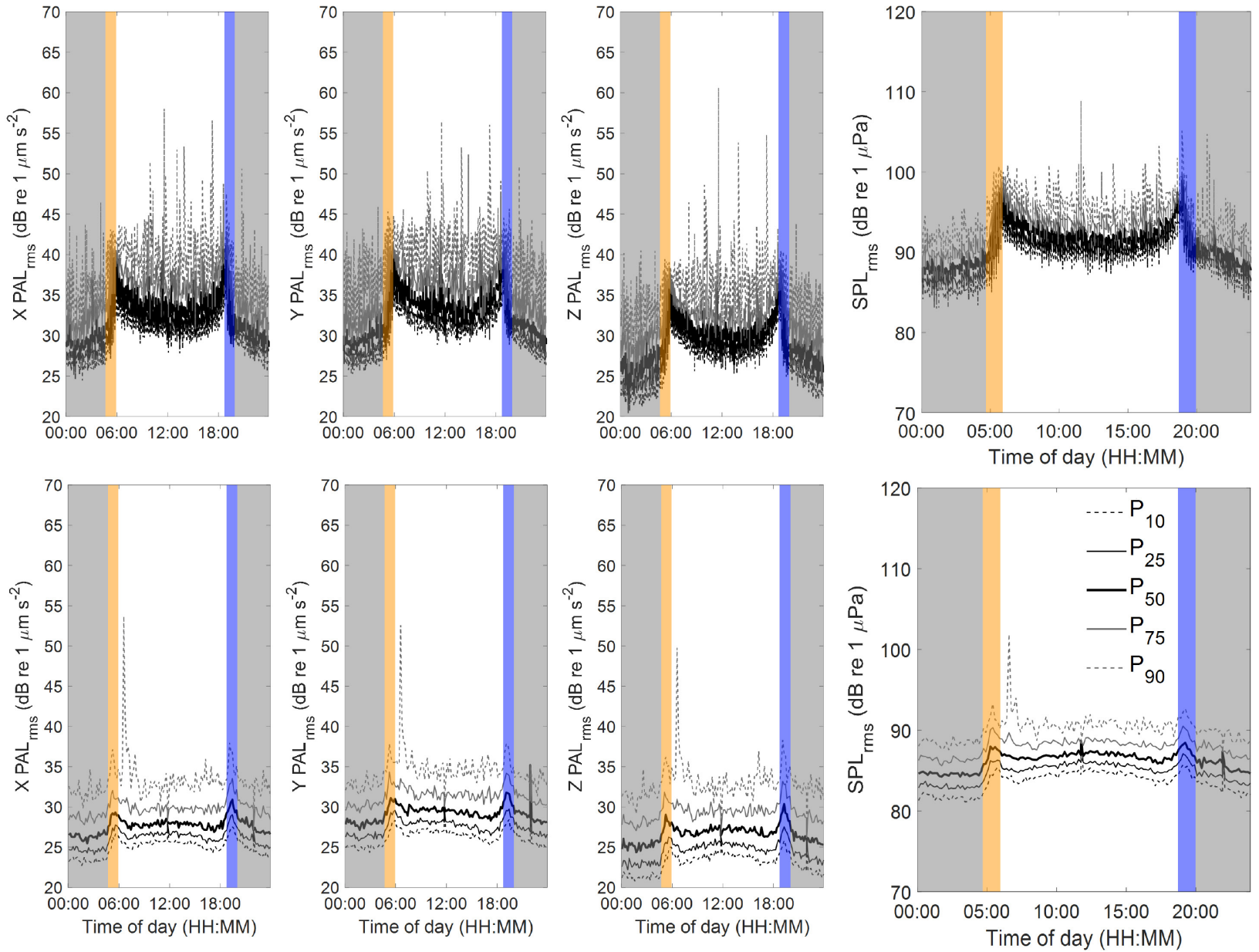

Figure S4. Diel trends as shown in Fig. 4, but for single-axis particle acceleration (X, Y, Z) and pressure (mean of all four hydrophones). The white background indicates day, gray background indicates night, and orange and purple bars indicate dawn and dusk respectively. 


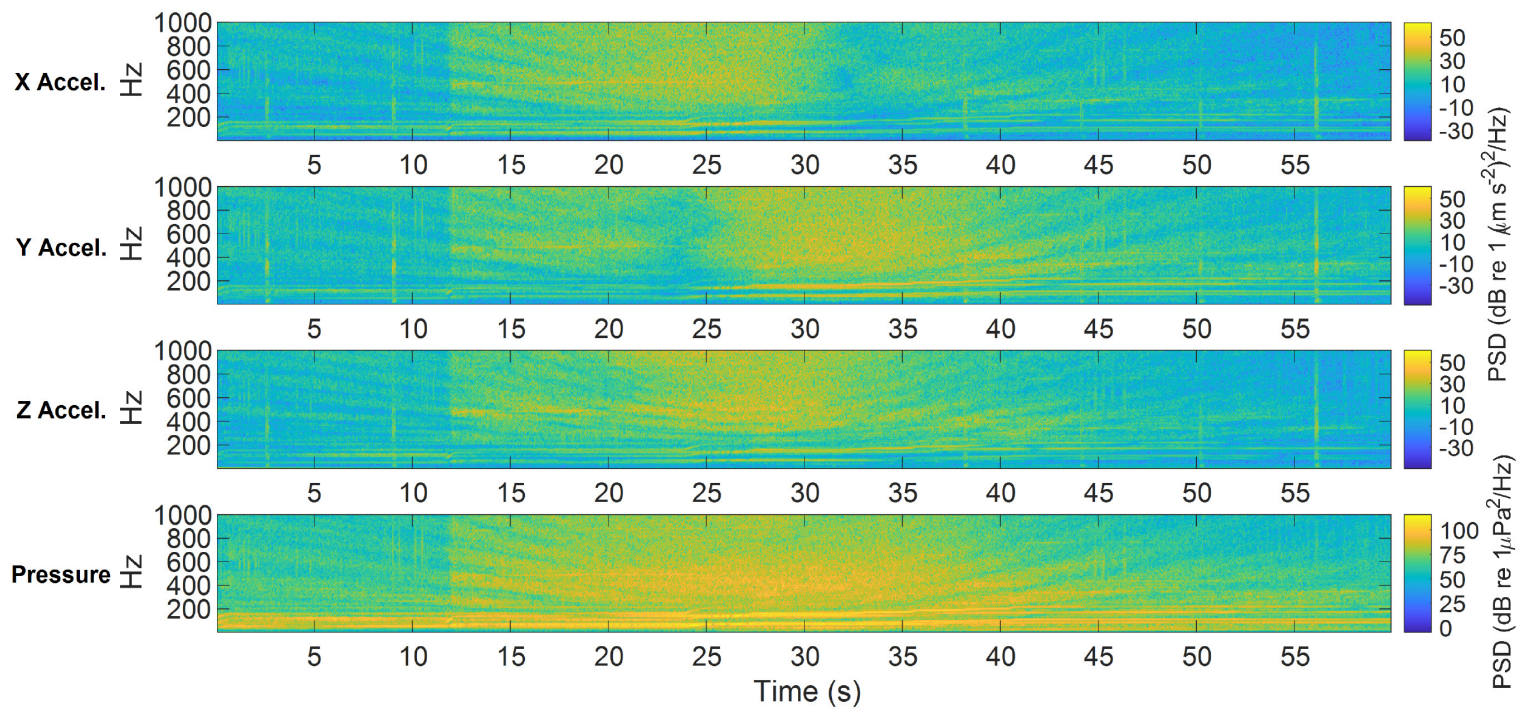

Figure S5. Single-axis particle acceleration $(X, Y, Z)$ and pressure for the example boat pass shown in Fig. $6 \mathrm{~b}$, recorded at Tektite on June $22^{\text {nd }} 2017$. 

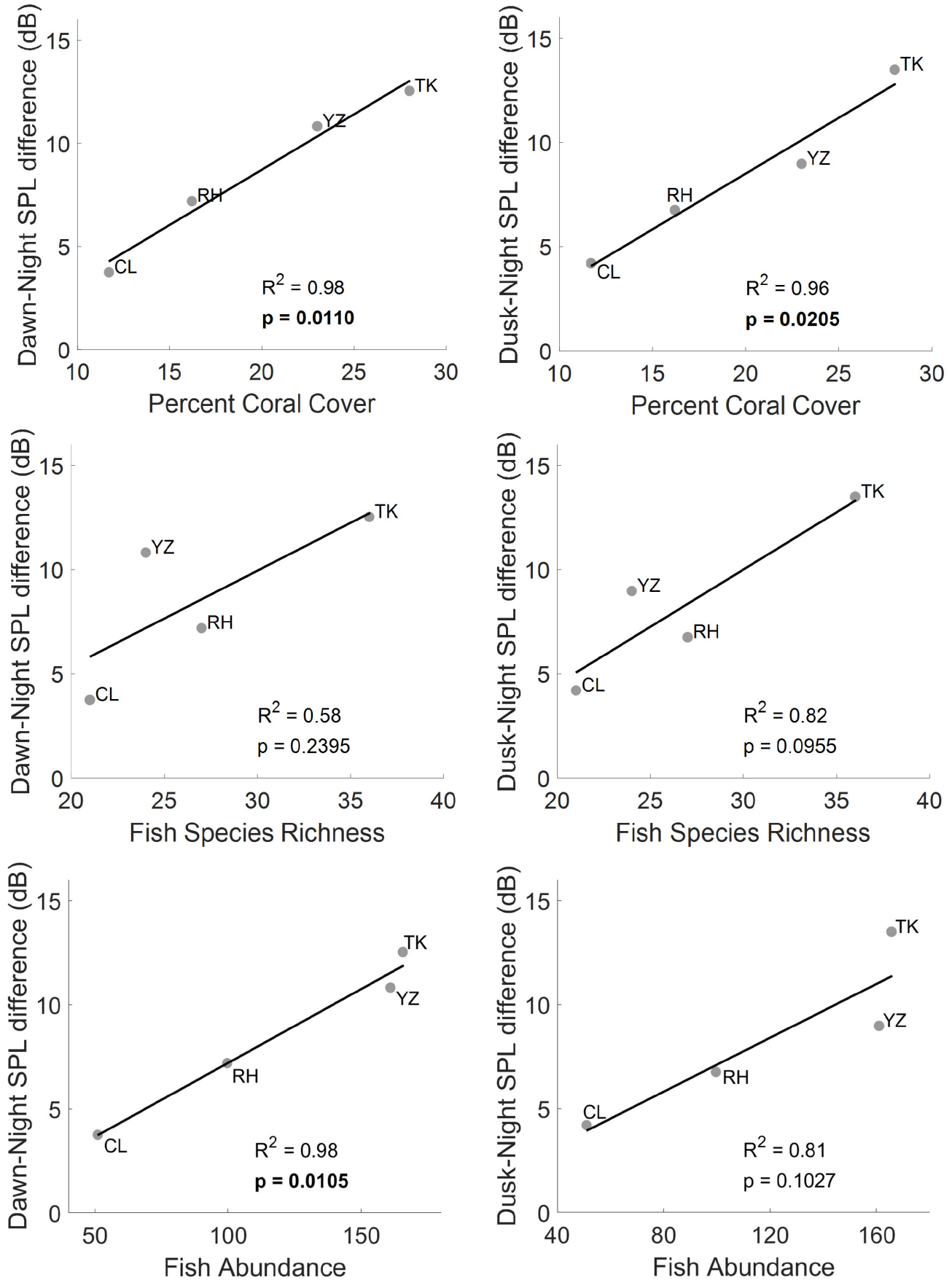

Figure S6. Diel strength of root-mean-square sound pressure level ( $\left.\mathrm{SPL}_{\mathrm{rms}}\right)$ at each site, of dawn peaks relative to night (left column) and dusk peaks relative to night (right column) versus visual survey metrics including percent coral cover, fish species richness, and fish abundance. $R^{2}$ and $p$ values were obtained from Pearson's correlation. Significant $p$ values $(p<0.05)$ are in bold. 


\section{APPENDIX E: COMPLEXITIES OF TANK ACOUSTICS WARRANT DIRECT, CAREFUL MEASUREMENT OF PARTICLE MOTION AND PRESSURE FOR BIOACOUSTICS STUDIES}

Reproduced from: Jones, I. T., Stanley, J. A., Bonnel, J., Mooney, T. A. (2019). Complexities of tank acoustics warrant direct, careful measurement of particle motion and pressure for bioacoustic studies. Proceedings of Meetings on Acoustics, 37, 010005. https://doi.org/10.1121/2.0001073, with the permission of AIP Publishing. 


\section{INTRODUCTION}

Sound propagation in confined spaces and near boundaries is often complicated and difficult to predict or model. Consequently, in situ measurements in the field and in tanks are vital for detailed and controlled monitoring of sounds produced by animals, and to address acoustically-mediated responses to sound exposures. For results from these studies to be accurately interpreted, it is vital that researchers understand the specificities of tank acoustics, and undertake thorough measurements and analyses of the acoustic field in terms of pressure and particle motion, particularly if studying an organism that detects either stimulus. All fish are considered to be sensitive to particle motion, as are many invertebrates; further, many fish and invertebrates are sensitive only to particle motion and not sound pressure (Mooney, Samson, et al., 2016; Popper \& Hawkins, 2018; Popper et al., 2001) making measurement of particle motion critical for bioacoustic studies on members of these taxa. Due to influences of tank boundaries on the acoustic field, particle motion cannot be easily calculated from pressure measurements in tanks. Rather, particle motion should be measured directly, or adequate models relating particle motion and pressure should be used for a confined space of interest (Gray et al., 2016; Nedelec et al., 2016). Equations used to estimate particle velocity and particle acceleration from point pressure measurements and pressure differentials between hydrophones are reviewed in Nedelec et al. (2016) and Gray et al. (2016). These equations are valid for plane waves (in which wave fronts are essentially flat), which exist far from a source and in the acoustic 'free-field' (where boundaries are absent). These conditions are not met in areas with reflective boundaries, including tanks and coastal waters. Accurate models for confined spaces require inputs for boundary conditions which are often unknown.

When accurate, empirically-supported models are not available for enclosed spaces, the necessity to directly measure acoustic pressure and particle motion is generally accepted. However, many bioacoustic studies do not adequately measure and report measurements of both sound pressure and particle motion in their study area. This leads to potential misconceptions and an often incomplete understanding of the magnitude, and spectral, 
temporal, and directional properties of acoustic signals animals in aquaria produce or are exposed to. Further, because variation of the sound field is not as easily predictable as in the free field, spatial measurements greatly support many behavioral and physiological measurements (Akamatsu et al., 2002). This paper focuses on empirical, high spatial resolution, direct measurement of acoustic pressure and particle motion fields in tanks for bioacoustic studies on fish and invertebrates. We provide examples of acoustic propagation and distortion phenomena of played-back pile driving sounds in a seawater tank. This tank was used for experiments investigating behavioral effects of exposure to played-back pile driving sound on squid (Doryteuthis pealeii).

\section{METHODS}

\section{Experimental tank and playback setup}

Acoustic recordings were made in a $1.1 \mathrm{~m}$ diameter, $0.6 \mathrm{~m}$ high, cylindrical, fiberglass tank filled to $0.5 \mathrm{~m}$ with seawater (Fig. 1). Animals were not present in the tank during recordings. The tank was above-ground and stood on layers of neoprene, plywood, and cement blocks to isolate the tank from ground vibrations. Audio playback was from a laptop connected to a Pyle PLA-2378 amplifier (Pyle Audio, Brooklyn, NY) powered by a $12 \mathrm{~V}$ battery. The amplifier powered a DNH Aqua-30 speaker (Theunissen Technical Trading, Malden, The Netherlands), which faced horizontally toward the center of the tank, suspended and centered at $25 \mathrm{~cm}$ deep, and $15 \mathrm{~cm}$ in front of the tank wall. A hydrophone (High-Tech Inc.) was suspended 1-2 cm from the tank wall at a horizontal distance of $44 \mathrm{~cm}$ from the speaker and at $35 \mathrm{~cm}$ deep; this hydrophone was used to monitor ambient noise during behavior experiments and was present, but not recording, during acoustic calibrations.

\section{Sound playback file}

The audio file used to calibrate the tank, and used in behavior experiments, was of offshore pile driving activity during construction of the Block Island Wind Farm (Rhode Island, USA) on October $25^{\text {th }}, 2015$. The pile was hollow and made of steel, had a diameter of 127.0 
$\mathrm{cm}$, wall thickness of $3.8 \mathrm{~cm}$, a rake of $13.27^{\circ}$ with respect to the vertical, and was driven up to $76.2 \mathrm{~m}$ deep into the seabed. The recording was from a hydrophone placed about $1 \mathrm{~m}$ above the seabed, and attached to a benthic sled $26 \mathrm{~m}$ deep and $500 \mathrm{~m}$ away from the pile (Amaral et al., 2018). A 1-min long file (from time 19:37 UTC) was used, with a sample rate of $9766 \mathrm{~Hz}$. The file was amplified in Adobe Audition (Adobe Systems, San Jose, California) by 11.5 dB to attain the highest possible playback amplitudes without clipping. Time in between pile driving strikes was edited to a fixed inter-strike-interval of $2 \mathrm{~s}$, resulting in a file of 30 pile strikes. The sound pressure amplitudes (zero-to-peak and time-integrated squared levels) of the 30 strikes were similar but not identical to avoid pseudoreplication of sound playback in the behavior experiments (standard deviation of zero-to-peak amplitudes: $\pm 0.3 \mathrm{~dB}$ ).

\section{Acoustic calibration of tank}

Playback of the 1-min pile driving sound file was recorded in a three-dimensional spatial grid in $10 \mathrm{~cm}$ increments, at four depths $(10,20,30$, and $40 \mathrm{~cm})$, yielding a total of 280 recording locations. The $X$ axis was assigned along the main axis of projection of the speaker, the $Y$ axis was perpendicular to the $X$ axis in the horizontal plane (Fig. 1), and the $Z$ axis was vertical. Particle acceleration and sound pressure were recorded at $48 \mathrm{kHz}$ sampling rates by a triaxial ICP accelerometer (Model W356B11, PCB Piezotronics) and Reson TC4013 hydrophone (Teledyne Marine) respectively, spaced $10 \mathrm{~cm}$ apart. The accelerometer had been sealed in a syntactic foam enclosure to make it waterproof and neutrally buoyant in seawater. All axes of the accelerometer had a flat frequency response (within $1 \mathrm{~dB}$ ) up to $7000 \mathrm{~Hz}$, and the hydrophone had a flat frequency receiving response (within $1 \mathrm{~dB}$ ) up to $10 \mathrm{kHz}$. The negative Zaxis side of the accelerometer was adhered with clear silicone to a narrow PVC pipe used as a probe. The accelerometer probe and hydrophone were suspended from a wooden board that lay across the top of the tank. The accelerometer was wired into a signal conditioner (Model 480B21, PCB Piezotronics) with unity gain. Single-ended signals from this conditioner and the hydrophone were input to two analog filters (Model 3382, Krohn-Hite Corporation), which each applied an anti-aliasing low-pass Butterworth filter at $24 \mathrm{kHz}$ and a gain of $20 \mathrm{~dB}$. The filter outputs were input to a data-acquisition board (USB 6251, National Instruments) which was 
connected to a laptop (X1 Carbon, Lenovo). The laptop ran a custom MATLAB (Mathworks, Natick, MA) script which started playback, and recorded and stored the voltage data from both transducers.

\section{Data analysis}

Acoustic data were analyzed using custom MATLAB scripts. Data were first de-trended (via subtraction of the mean) to remove DC offsets, then corrected to pressure and acceleration units ( $\mu \mathrm{Pa}$ and $\mu \mathrm{m} \mathrm{s}^{-2}$, respectively) by multiplying each sample of voltage data by nominal sensitivity values (accounting for gain applied) provided by the transducer manufacturers. All data, except those used in spectrograms or specified otherwise, were band-pass filtered to 20 $1000 \mathrm{~Hz}$ with an $8^{\text {th }}$ order Butterworth filter. Sound levels were calculated at each recording location in the tank for each pile strike, in time windows ranging $0.15 \mathrm{~s}$ before to $1 \mathrm{~s}$ after the time at which each strike peak was detected. Time-integrated squared (aka single-strike energy) levels of the pressure $\left(E_{p}\right)$ and particle acceleration $\left(E_{a}\right)$ were calculated by summing the square of their respective time series from samples spanning $5 \%$ to $95 \%$ of the cumulative energy curve (aka the pulse duration) of each pile strike, and normalizing this by the sample rate, using the following equation:

$$
E_{p} \text { or } E_{a}=10 * \log _{10}\left(\frac{\sum_{t=1}^{T_{90}} s_{t}^{2}}{f s}\right)
$$

where $S_{t}$ is the pressure $(\mu \mathrm{Pa})$ or particle acceleration $\left(\mu \mathrm{m} \mathrm{s}^{-2}\right)$ at a given time point $t, T_{90}$ is the pulse duration, and $f s$ is the sample rate in $\mathrm{Hz}$. $E_{p}$ and $E_{a}$ are reported in $d B$ re $1 \mu \mathrm{Pa}^{2} s$ and $d B$ re $1\left(\mu \mathrm{m} \mathrm{s}^{-2}\right)^{2} \mathrm{~s}$, respectively, and are hereafter referred to as "pressure energy" or "particle acceleration energy" respectively, or "energy" if referring to either $E_{p}$ or $E_{a}$. Since the particle acceleration is a time-dependent 3D vector, we described its (time-dependent) magnitude using the Euclidian norm, i.e. the square root of the sum of its squared spatial components: $X$, $Y$, and $Z$. The particle acceleration energy $E_{a}$ was calculated separately for the three components, and also for the acceleration magnitude (i.e. the Euclidian norm of the acceleration). Unless otherwise specified, $E_{a}$ is reported for the acceleration magnitude 
hereafter. Parameters used to compute spectral density (in $\mathrm{dB}$ re $1 \mu \mathrm{Pa}^{2} / \mathrm{Hz}$ ) for spectrograms were as follows: window length: $0.033 \mathrm{~s}$ (Hann window); window overlap: $50 \%$; FFT size: $1 / 5^{\text {th }}$ of the sample rate. This yielded a frequency resolution of $5 \mathrm{~Hz}$ and temporal resolution of about $0.17 \mathrm{~s}$.

\section{RESULTS AND DISCUSSION}

Broadband results: patterns and relationships between pressure and particle acceleration magnitudes

Substantial variability of pressure and particle acceleration across recording locations in the tank was found, especially throughout the XY plane at a given depth (Fig. 2). For each metric, the pattern in the $X Y$ plane was relatively consistent across depths, though absolute magnitudes of pressure and particle acceleration energy were greater at 20 and $30 \mathrm{~cm}$ depths than 10 and $40 \mathrm{~cm}$ depths. This may be due to these depths being closer to the depth the speaker was centered at $(25 \mathrm{~cm})$. Particle acceleration from the speaker projected predominantly along the $\mathrm{X}$ axis, as indicated by higher magnitude energy in the $\mathrm{X}$ axis alone compared to the $\mathrm{Y}$ and $\mathrm{Z}$ axes alone at most locations (not shown).

In this broadband range analyzed from $20-1000 \mathrm{~Hz}$, neither pressure nor particle acceleration followed a monotonic relationship with horizontal distance from the speaker, which is apparent in Fig. 2 and also can be seen in Fig. 3, in which dB levels are plotted along a transect on the $X$ axis at $Y=0 \mathrm{~cm}$, relative to the highest $d B$ level along the transect. At each depth, sound pressure energy was higher closer to the speaker (at $X=15 \mathrm{~cm}$ ) and near the tank walls (e.g. $X=85 \mathrm{~cm})$, and lower near the center of the tank $(X=35-55 \mathrm{~cm})$.

Unlike the pattern seen in this broadband analysis, pressure in theory is assumed to approach zero at tank walls, for tanks that are above-ground with a low-impedance medium (e.g., air) on the other side of the wall (Gray et al., 2016; Parvulescu, 1964; Rogers et al., 2016). This condition is a reasonable assumption in tanks with glass or plastic walls where the wall thickness is far less than the acoustic wavelength of water, and importantly, for frequencies far from resonant frequencies (Gray et al., 2016). Also, according to empirical data and models 
from Akamatsu et al. (2002), spectral levels (in $\mathrm{dB}$ re $1 \mu \mathrm{Pa}^{2}$ ) of frequencies well below the minimum resonant frequency of the tank will exponentially decrease with distance from the tank; this is also apparent for $E_{p}$ in Fig. 2 at least for $Z=10,20$, and $30 \mathrm{~cm}$, but only up until 45 $\mathrm{cm}$ away from the speaker, after which $E_{p}$ increases again with distance. Note that at $Y$ positions nearest the tank walls, the hydrophone and accelerometer were within $5 \mathrm{~cm}$ of the tank wall, and often within $1 \mathrm{~cm}$ of the wall on $\mathrm{X}$ transects away from those spanning close to the full diameter of the tank $(X<35$ or $X>45 \mathrm{~cm})$. These results suggest the broadband range from $20-1000 \mathrm{~Hz}$ included resonant frequencies (discussed in more detail in the next session). The pattern of particle acceleration with distance from the speaker was more complicated compared to that of pressure. The particle acceleration was relatively high in magnitude near the speaker $(X=15 \mathrm{~cm})$ and near the tank walls (similar to the pressure data), but unlike pressure, it also peaked near the center of the tank. The $X$ position of this peak was not consistent across transects at different $Y$ positions. Even with the spatial recording resolution of $10 \mathrm{~cm}$, large differences (up to $9 \mathrm{~dB}$ ) existed between many adjacent recording locations. Consistent with the theory that particle motion magnitude is enhanced near tank boundaries (Gray et al., 2016; Rogers et al., 2016), the highest particle acceleration magnitudes were found near tank walls (e.g. $X=25 ; Y=50 ; Z=20$ ). In theory particle motion should also be enhanced near the tank surface and tank bottom, though recording locations in the present study may not have been close enough to these boundaries to record these maxima.

Importantly, these data reinforce the concept that particle motion cannot be accurately predicted from pressure measurements in tanks if one uses simplistic plane wave models. Rather, particle motion should be measured directly, in the absence of more complex models for enclosed environments. The distinctly different spatial patterns of particle motion compared to pressure as seen in Fig. 2-3 indicate pressure and particle acceleration did not have a simple proportionality to each other with distance from the speaker. To reiterate, this result is to be expected in any enclosed body of water with nearby boundaries present, shallow water environments, and close to the surface, seabed and other structures such as reefs. These are situations in which far field and plane wave conditions cannot be assumed (Nedelec et al., 2016). In many locations and experimental regimes, fine spatial-scale, direct measurements of 
both pressure and particle acceleration are therefore fundamental to describing sound treatments, especially for particle motion sensitive animals.

\section{Resonant frequencies and reverberation}

Due to the structural properties of tank walls, such enclosures will have natural frequencies of vibration, called resonant frequencies, that can be overexpressed relative to other frequencies if signals played or recorded in the tank include frequencies close to those resonant frequencies. In confined environments such as tanks, spectral levels at frequencies that are well below the minimum resonant frequency, and that are below a defined 'cut-off' frequency (frequencies below the cut-off do not propagate well in shallow water), are expected to decay exponentially with distance from the sound source. Conversely, spectral levels at and near resonant frequencies are expected to be relatively constant throughout the tank (Akamatsu et al., 2002). According to equations from Akamatsu et al. (2002) for cylindrical tanks, the theoretical cut-off frequency for our experiment tank is $1500 \mathrm{~Hz}$, and the theoretical minimum resonant frequency is about $1533 \mathrm{~Hz}$, assuming a sound speed in the seawater tank of $1500 \mathrm{~m} \mathrm{~s}^{-1}$. If there were indeed no resonant frequencies below $1500 \mathrm{~Hz}$ in our tank, we would expect sound pressure energy at all frequencies in the $20-1000 \mathrm{~Hz}$ band to decay exponentially from the source and would not expect the observed enhanced sound pressure energy near the tank walls (Fig 2-3).

Efforts to determine the exact resonant frequencies in our experiment tank yielded inconclusive results, likely in part due to distortion of the frequency spectrum by the speaker. The pile driving impulses in the original playback file had high signal energy below $1000 \mathrm{~Hz}$ (highest from 100-300 Hz), and relatively constant energy between 1000 and $4500 \mathrm{~Hz}$ (Fig. 4A). Comparatively, spectrograms from in-tank recordings for pressure and all three axes of particle acceleration revealed signal energy was over-expressed between 100-300 Hz and above 2000 $\mathrm{Hz}$, and under-expressed between $500-1500 \mathrm{~Hz}$ (Fig. 4B-E). Thus, if a resonant frequency existed near $1500 \mathrm{~Hz}$, the apparent lower output of the speaker at this frequency may have prevented contamination of the received signal in the tank by this resonant frequency. The non-flat, nonmonotonic frequency response of the speaker (as reported by the manufacturer) likely heavily 
distorted the spectral content of signals received in the tank, as the change in spectral density levels (in $\mathrm{dB}$ re $1 \mu \mathrm{Pa}^{2} / \mathrm{Hz}$ ) across frequencies followed the speakers' reported frequency response: sound pressure level of the Aqua-30 speaker response was reported to steeply increase by $45 \mathrm{~dB}$ from 20 to $150 \mathrm{~Hz}$, decrease by $10 \mathrm{~dB}$ from $150 \mathrm{~Hz}$ to the range of 700-2000 $\mathrm{Hz}$, and increase by $5 \mathrm{~dB}$ from 2000-5000 Hz (DNH, n.d.).

Analyses shown in Fig. 2 were repeated after filtering data to a 1 octave band centered on $250 \mathrm{~Hz}$, and also a 10-Hz wide band centered on $80 \mathrm{~Hz}$. Both frequency bands yielded similar patterns of sound pressure and particle acceleration compared to the $20-1000 \mathrm{~Hz}$ band, with increases in signal energy near the tank walls (not shown). However, repeating this analysis on a $10-\mathrm{Hz}$ wide band centered at $800 \mathrm{~Hz}$ revealed sound pressure fields at each depth that decreased in energy to the tank walls without increasing again (Fig. 5). Thus, a pressure-release condition at the tank walls was apparent at this non-resonant frequency, and the increase in pressure approaching the edges of the tank seen in Fig. 2 appears to primarily occur between about $100-300 \mathrm{~Hz}$. Within this range, flexural (bending) resonant frequencies may exist. At and near flexural frequencies, thin tank walls (even those much thinner than the shortest wavelength of interest, as is the case in this study) can have resonance effects on the interior tank sound field (Rogers et al., 2016). Importantly, the particle acceleration at $800 \mathrm{~Hz}$ still was not proportional to sound pressure and did not follow a monotonic relationship with horizontal distance from the speaker. Differences in spatial patterns observed in Fig. 2 vs. Fig. 5 illustrate how the selection of a frequency band can have major impacts on the analysis and interpretation of the acoustic field in tanks; bands including resonant frequencies can show very different patterns from bands excluding resonant frequencies.

In the $20-1000 \mathrm{~Hz}$ band there was evidence of reverberation, visible in the spectrograms as 'temporal smear' of the pile strikes (Fig. 4) and in the waveforms (Fig. 6). This reverberation was diffuse, since discrete echoes of the signal could not be resolved. Reverberation was stronger (i.e., there was greater temporal lag in the waveform) at the sides of the tank compared to the center. Comparing the time series of pressure at one location near the tank wall to one location near the center (both at $X=25$ and $Z=20 \mathrm{~cm}$ ), the tank wall location had more lag relative to the original playback file $(18.12 \mathrm{~ms})$ compared to the lag of the center 
location relative to the original playback file $(5.12 \mathrm{~ms})$. The waveform at the tank side location lagged by 8.09 ms relative to the tank center location. This demonstrates how tank geometry can cause the energy of emitted impulses to spread out over a longer time period, especially near boundaries.

\section{Directionality of particle acceleration}

Particle motion is inherently directional, and evidence suggests it is utilized by fish and invertebrates as a sound-localization cue (Popper \& Hawkins, 2018; Wilson et al., 2018; Zeddies et al., 2012). Therefore, it is important to consider the directional fields of particle motion in tanks used in fish and invertebrate bioacoustic studies, in addition to particle motion magnitudes. Phase of the particle motion must be taken into account here. If using an accelerometer, one must keep track of the positive and negative ends (designated by the manufacturer). For a given axis, acceleration above zero on the waveform denotes motion in the positive direction along that axis and acceleration below zero denotes motion in the negative direction. Particle motion vector fields of transient signals should be plotted at instantaneous times, i.e. at the same time sample points for all axes, to preserve the correct phase relationships between axes.

Efforts were made to visualize the vector fields of particle acceleration in our tank, both in $2 \mathrm{D}$ in the $\mathrm{XY}$ plane at four depths and in $3 \mathrm{D}$. However, we discovered that the starting time points of recordings were not synced with starting times of playback. In other words, playback was not controlled to begin at precisely the same time after the start of recording, rather the time between these differed by a variable fraction of a second among recording locations. Due to these phase discrepancies across recording locations, the vector field as a whole could not be accurately visualized. Cross-correlating and syncing waveforms between locations to align pile impulses to start at the same time point would not be appropriate, since this would remove the 'true' time delays of impulses that are recorded at locations further from the speaker compared to locations closer to the speaker. This reinforces the critical need for timing of initiating playbacks and recordings to be tightly synced across recording locations in order to characterize vector fields in tanks. 


\section{CONCLUSIONS AND RECCOMMENDATIONS}

These data from fine-scale spatial mapping of in-tank acoustic propagation reveal several key properties of pressure and particle motion in tanks and demonstrate precautions that should be taken when conducting and reporting tank acoustic measurements. These ideas are not novel, rather they have been stressed in past publications reporting empirical and modeled tank acoustic data (Akamatsu et al., 2002; Gray et al., 2016; Jézéquel et al., 2019; Rogers et al., 2016). Our empirical dataset serves as reinforcement of the following important reminders and recommendations bioacousticians should consider when conducting in-tank experiments with particle-motion sensitive aquatic organisms:

- Influences of tank resonance and frequency responses of the speaker (if a playback experiment is conducted) and receiving transducers (e.g. hydrophones and accelerometers) need to be considered and reported, as these will distort played and recorded power spectra of acoustic signals. Further, resulting spatial patterns of pressure and particle motion magnitudes will differ when including versus excluding frequencies near resonant frequencies in analyses.

- Reverberation by tank boundaries is inevitable to some extent, and this will distort signals. This distortion will often exist as diffuse reverberation in which multiple reflections of a signal overlap in time so that discrete echoes in the waveform cannot be resolved.

- To accurately report the directionality of particle motion in tanks, care must be taken to sync recordings across recording locations so that phases are properly temporally aligned; this is especially true for transient signals and at higher frequencies where phase of the particle motion will change faster and over shorter time scales.

- Results presented herein indicate that particle motion (whether displacement, velocity, or acceleration) cannot be easily predicted from sound pressure in tanks using calculations assuming free-field and plane wave conditions, and pressure and particle motion will not have an easily predictable proportionality to each other. In absence of accurate, empirically supported propagation models for specific confined spaces, particle motion should be directly measured, such as with accelerometers or geophones. 


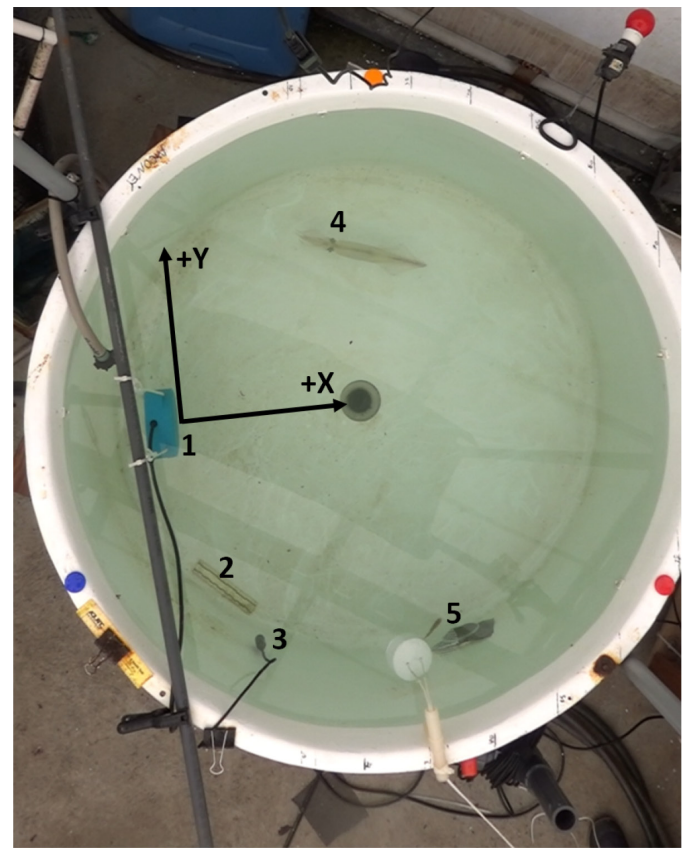

Figure 1. Top-down view of experiment tank, showing (1) underwater speaker, (2) ruler used for calibration of squids' depth in the tank, (3) hydrophone, (4) squid (Doryteuthis pealeii), and (5) killifish (Fundulus heteroclitus), which was released from a PVC container during feeding behavior experiments (the squid and killifish were not present during tank calibrations, but all other materials shown were present). Positive $X$ and $Y$ axes with respect to speaker orientation are shown; the positive $Z$ axis points upwards away from the tank bottom. The front (right) edge of the speaker marks the origin of the $\mathrm{XY}$ plane. 

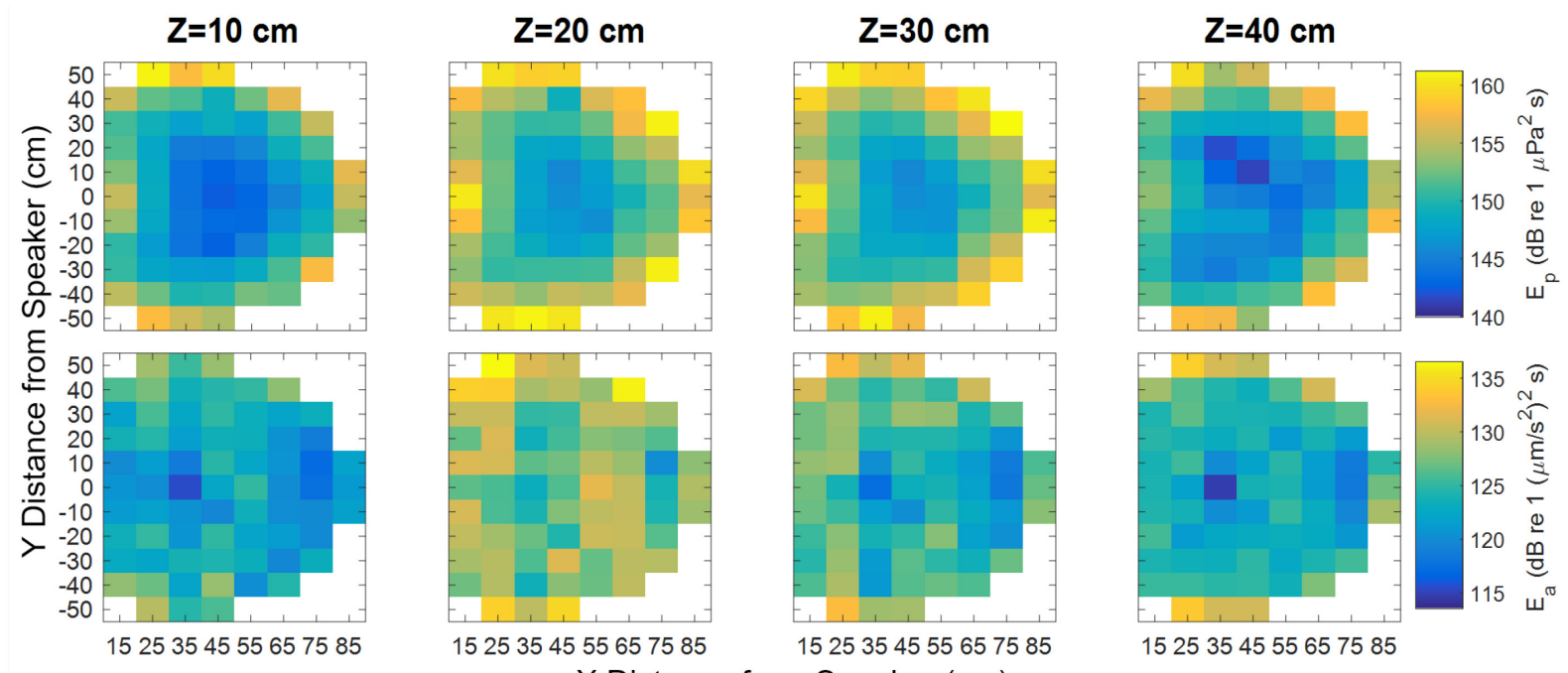

1525354555657585

1525354555657585 $\mathrm{X}$ Distance from Speaker $(\mathrm{cm})$

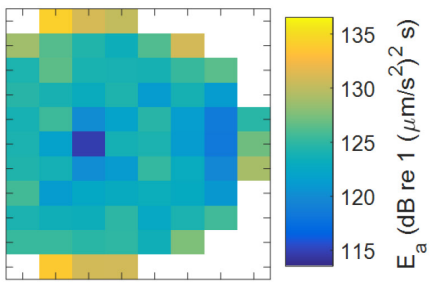

1525354555657585

Figure 2. Time-integrated squared levels of pressure (top row, $E_{p}$ ) and acceleration (bottom row, $E_{a}$ ) in horizontal XY planes at four different depths (columns). Each block represents a median value of 30 pile strikes at one recording location. Data shown were band-pass filtered between 20 and $1000 \mathrm{~Hz}$. 

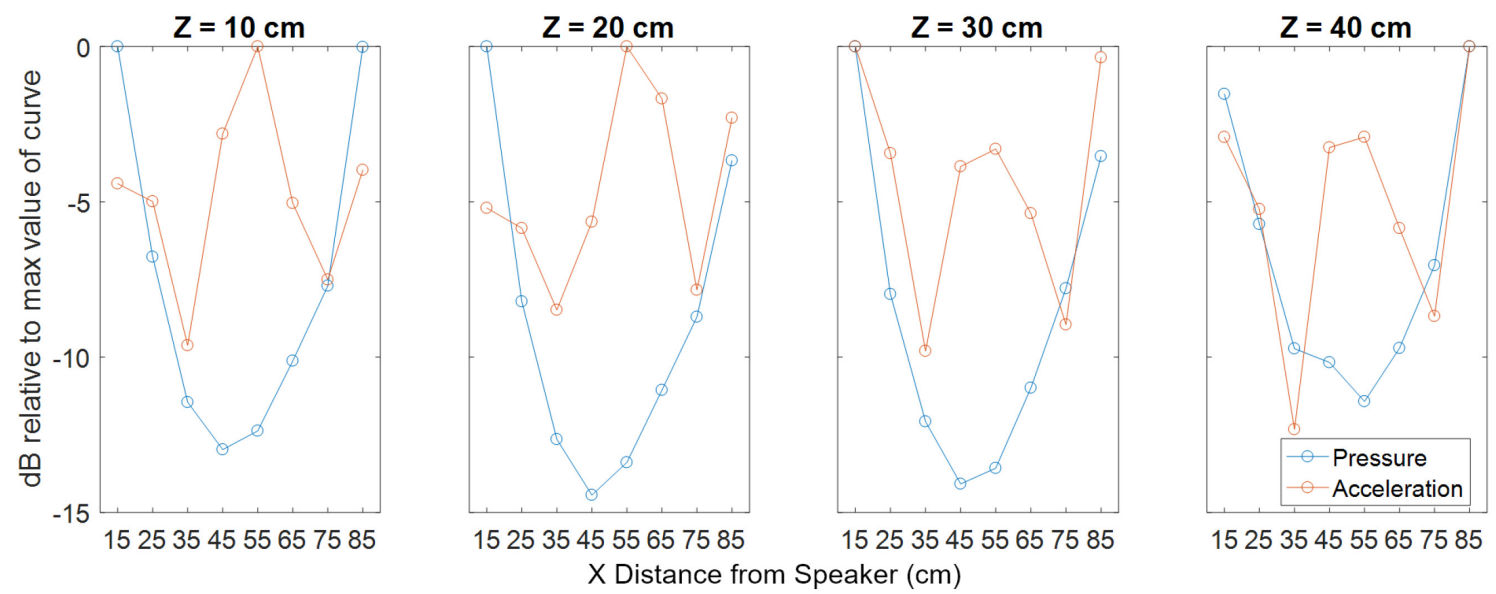

Figure 3. Pressure and acceleration energy levels from Fig. 2 (in units of $d B$ re $1 \mu \mathrm{Pa}^{2} s$ and $d B$ re 1 $\left(\mu \mathrm{m} \mathrm{s}^{-2}\right)^{2} \mathrm{~s}$, respectively) along a transect on the $X$ axis at $Y=0$, for four depths. Note that pressure and acceleration have non-monotonic relationships with distance from the speaker, and that pressure and acceleration have different patterns from each other with distance from the speaker. 

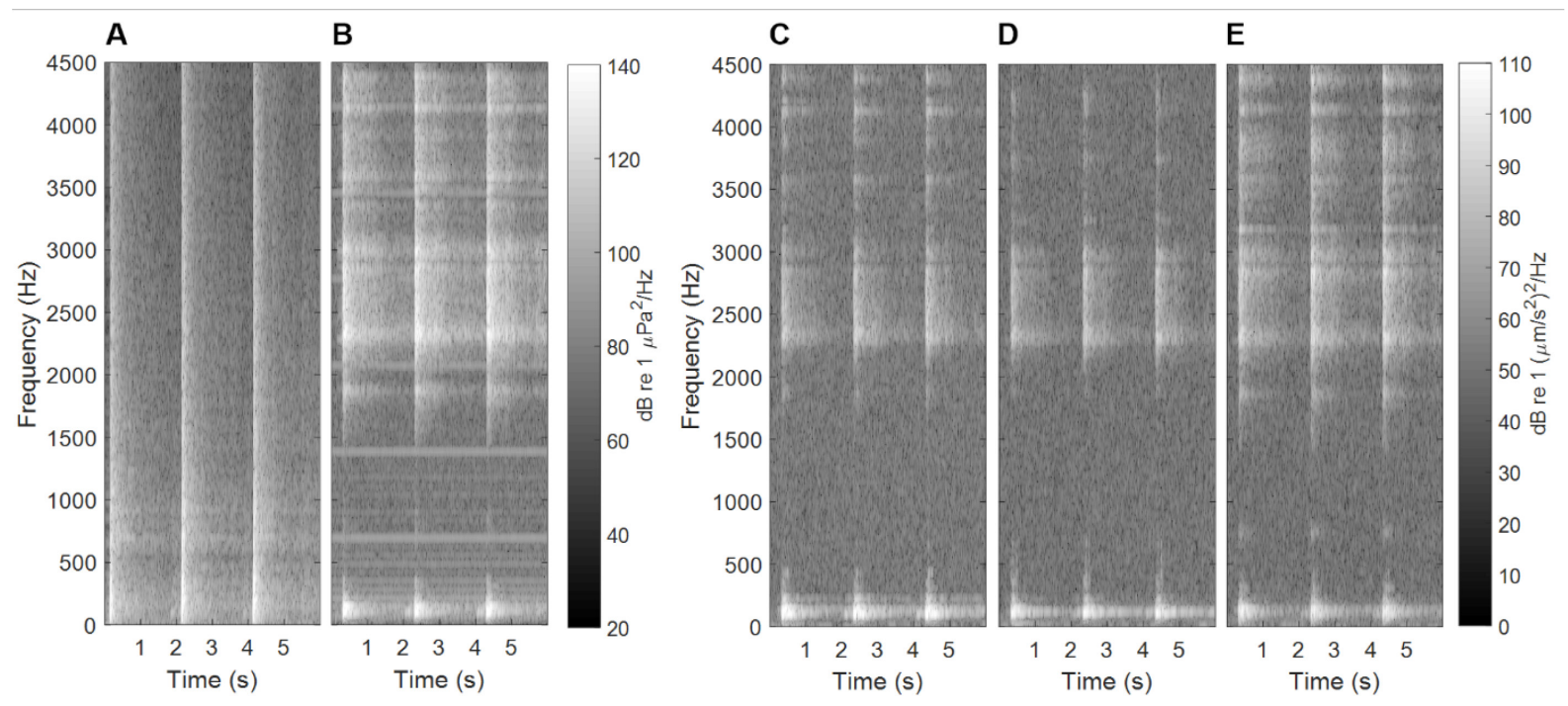

Figure 4. Spectrograms of the first 3 pile strikes, in A) field recording of pressure used for playbacks, B) pressure at one location in the tank $(X=45 ; Y=0 ; Z=20), C-E)$ particle acceleration at the same location in the $X, Y$, and $Z$ axes, respectively. All spectrograms have $5 \mathrm{~Hz}$ frequency bins and $0.17 \mathrm{~s}$ time bins ( 0.33 s time windows with $50 \%$ overlap). Narrow-band continuous tones present in $B$ are likely electrical artifacts from the recording system. 

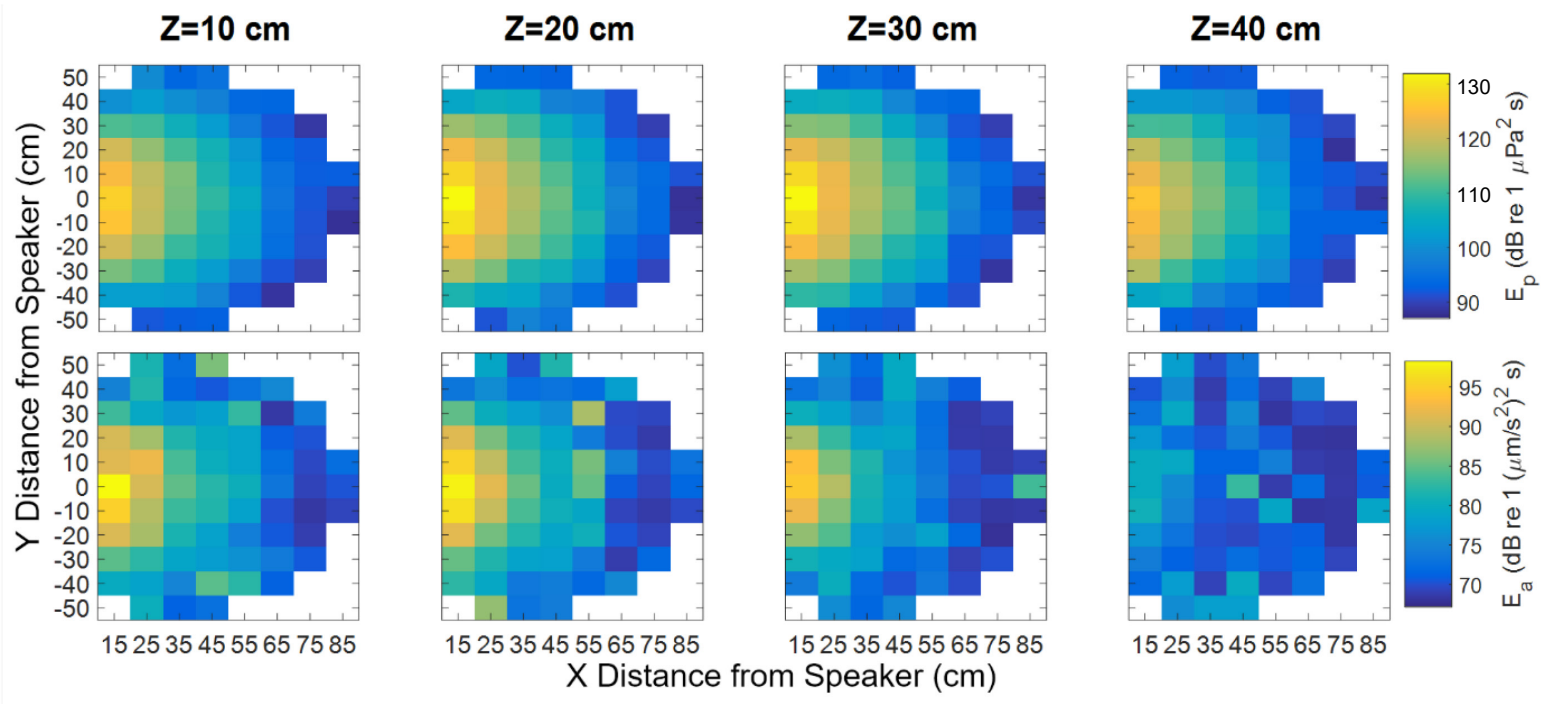

Figure 5. Time-integrated squared levels of pressure and particle acceleration as shown in Fig. 2, but instead after filtering data to a $10-\mathrm{Hz}$ wide band centered on $800 \mathrm{~Hz}$ ( $8^{\text {th }}$ order Butterworth filter). 

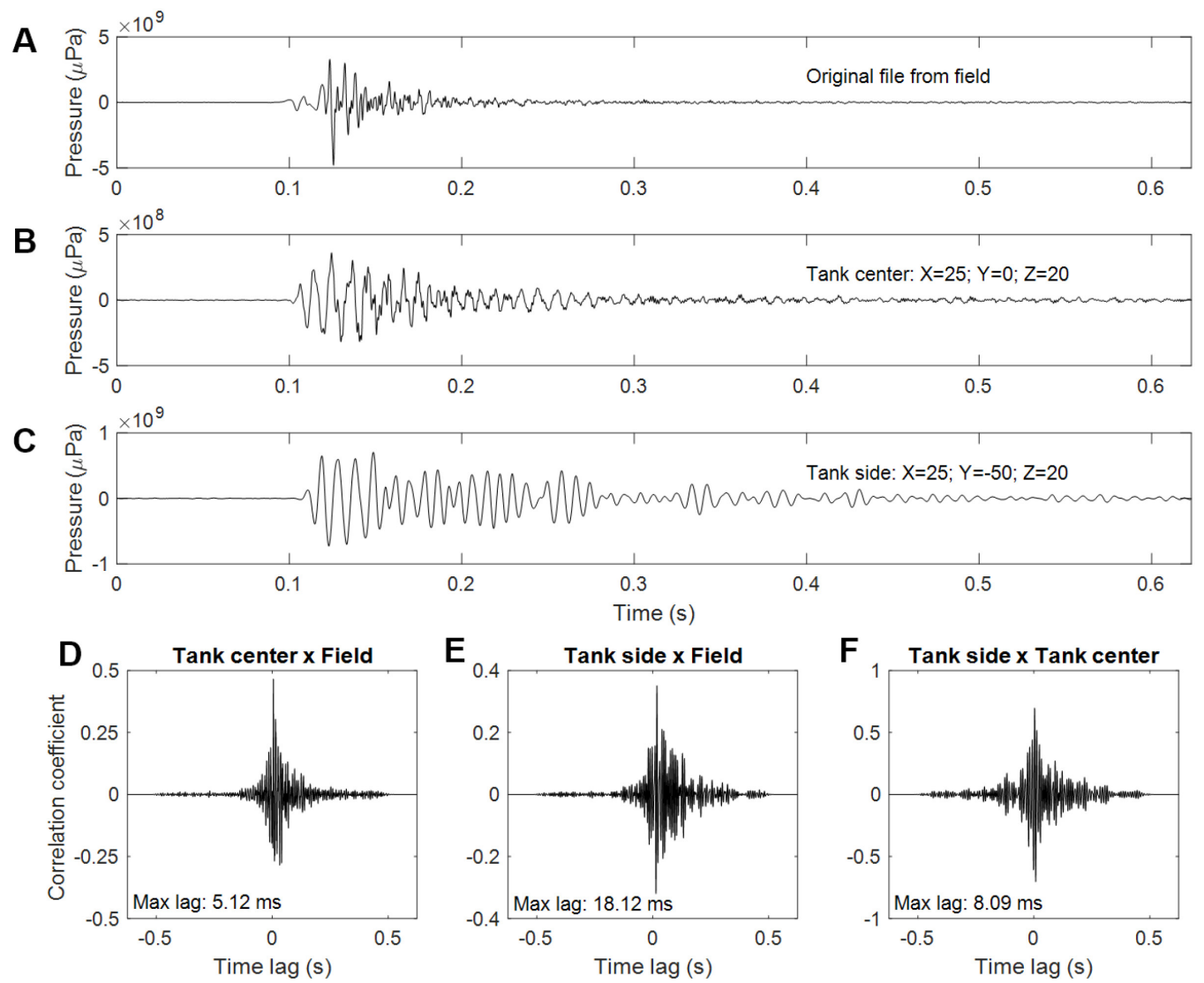

Figure 6. Acoustic pressure waveforms and cross-correlation of waveforms for the first strike only of the 1-min pile driving sound file. A) Waveform of the original field recording, B) waveform from one location near the center of the tank $(X=25 ; Y=0 ; Z=20 \mathrm{~cm}), C$ ) waveform from one location near the tank side-wall $(X=25 ; Y=50 ; Z=20 \mathrm{~cm}), D-F)$ cross-correlation between the waveforms in $B$ and $A, C$ and $A$, and $C$ and $B$, respectively. "Max lag" indicates the time delay in $m s$, i.e. the point at which the cross-correlation coefficient is maximum. In D-F, positive time delays indicate the first location listed in the subplot title had a waveform delayed in time relative to the second location listed, i.e. the waveform in the first location listed was more strongly distorted by reverberation. All data shown were filtered to $20-1000 \mathrm{~Hz}$. 
APPENDIX F: EXAMPLE MATLAB CODE USED TO CALCULATE PARTICLE ACCELERATION FROM ACCELEROMETERS AND HYDROPHONE ARRAYS 


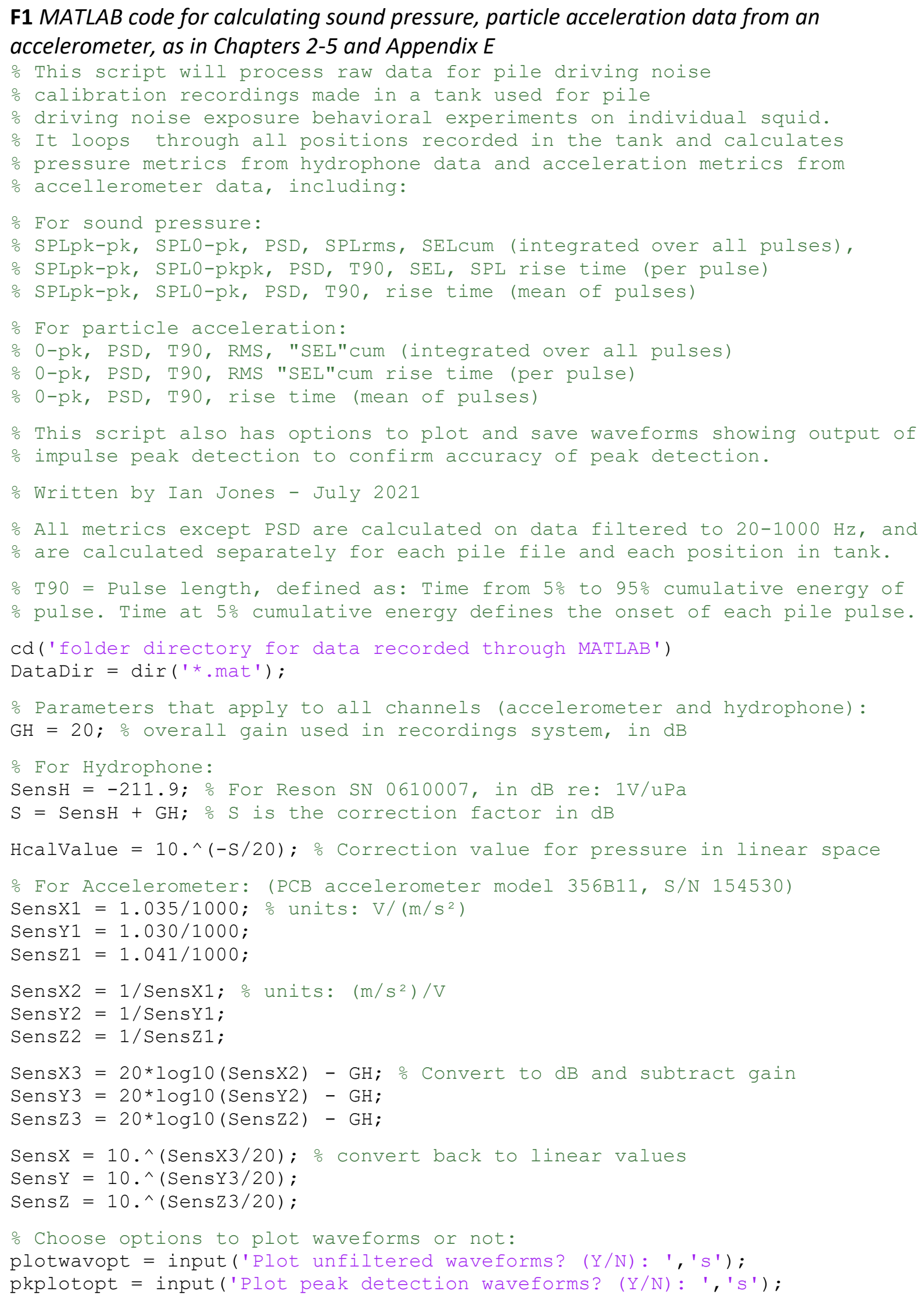




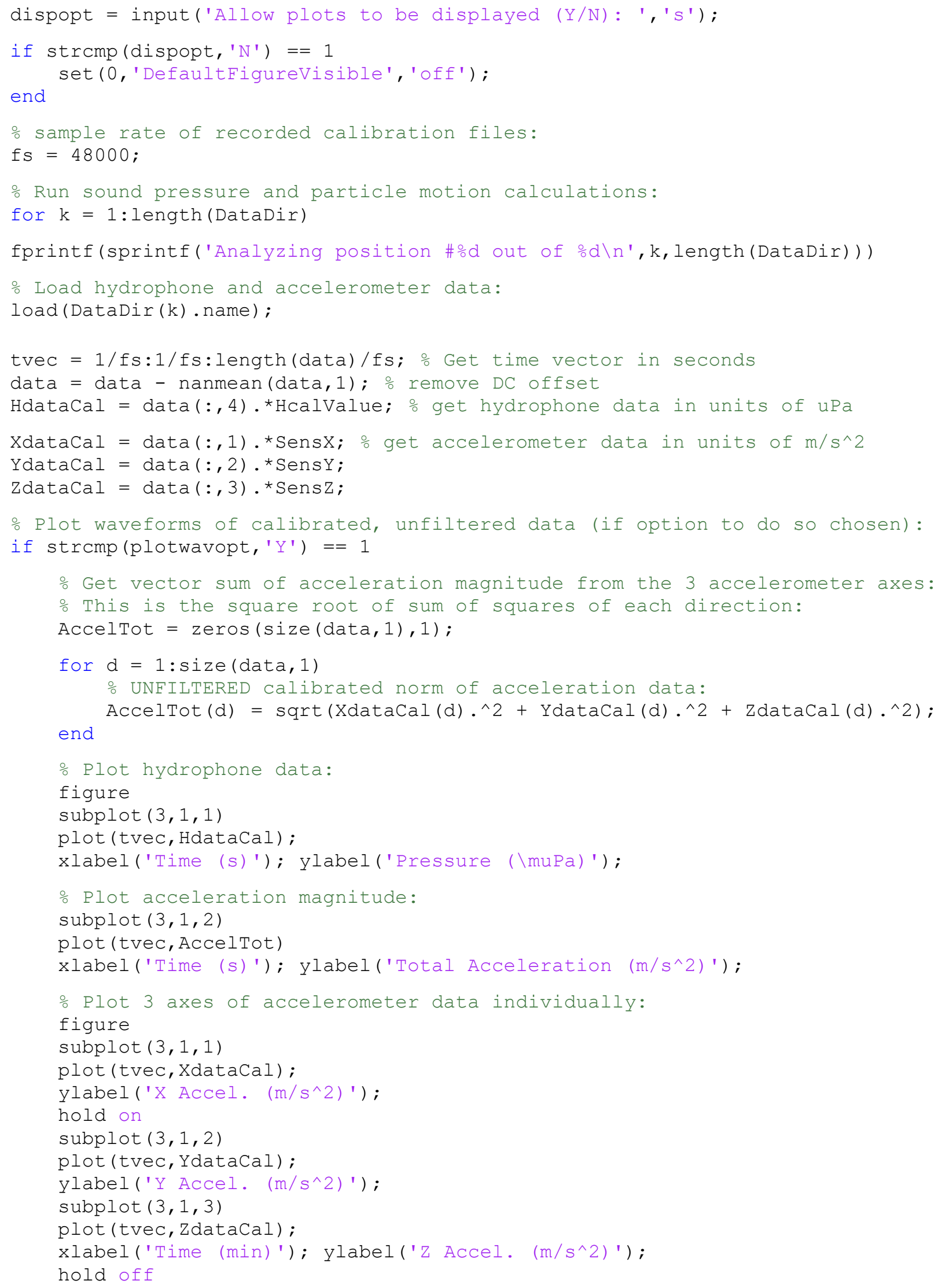


close all

end

\% Design Butterworth filter (becomes 8th order, after using filtfilt)

Fpass $1=20 /(\mathrm{fs} / 2) ;$ Lower $-3 \mathrm{~dB}$ cutoff

Fpass2 = $1000 /(\mathrm{fs} / 2) ;$ Upper $-3 \mathrm{~dB}$ cutoff

DFilter = fdesign.bandpass ('N,F3dB1,F3dB2', 4,Fpass1,Fpass2);

bbp = design(DFilter,'butter'); butterworth bandpass filter

\% freqz(bbp, fs, fs) \% Uncomment to visualize the filter

․ Final calibrated, zero-phase filtered pressure data:

HdataFilt = filtfilt(bbp.sosMatrix,bop.ScaleValues, HdataCal);

o Zero-phase filtered data for each accelerometer axis: :

Axfilt = filtfilt(bop.sosMatrix,bop.ScaleValues, XdataCal);

AYfilt = filtfilt(bbp.sosMatrix,bop.ScaleValues, YdataCal);

Azfilt = filtfilt(bop.sosMatrix,bbp.ScaleValues, ZdataCal);

\% Get vector sum of acceleration magnitude from the 3 accelerometer axes:

o This is the square root of sum of squares of each direction:

AdataFull = $\operatorname{zeros}(\operatorname{size}(\operatorname{data}, 1), 1)$;

for $d=1: \operatorname{size}(\operatorname{data}, 1)$

oAdataFull is the final calibrated, zero-phase filtered acceleration data:

Adatafull $(d)=\operatorname{sqrt}\left(\operatorname{AXfilt}(d) \cdot{ }^{\wedge} 2+\operatorname{AYfilt}(d) \cdot{ }^{\wedge} 2+\operatorname{AZfilt}(d) \cdot{ }^{\wedge} 2\right) ;$

end

o Split full into the three r min segments, omitting the partial

\% pile impulses at the very end of pilel and pile2 segments.

\% Note that Pilel is necessarily 0.20 s shorter than Pile2 and Pile3.

tvecP1 = tvec $($ tvec $<62.8) ;$ in seconds

svecP1 = $1:$ length (tvecP1); 응 samples

tvecP2 = tvec (tvec $>64 \&$ tvec $<127) ;$ in seconds

svecP2 = (svecP1 (end) $+\mathrm{fs}):($ svecP1 (end) $+\mathrm{fs})+$ length (tvecP2); in samples

tvecP3 = tvec $(128.2<$ tvec $) ; \%$ in seconds

svecP3 = ( svecP2 (end $)+(2 * f s)):(\operatorname{svecP} 2($ end $)+f s)+l e n g t h($ tvecP3 $) ; \%$ in samples

svec cell = SvecP1; svecP2; svecP3 $\}$;

tvec_cell $=\{\mathrm{svecP} 1 / \mathrm{fs} ; \mathrm{svecP} 2 / \mathrm{fs} ; \mathrm{svecP} 3 / \mathrm{fs}\}$;

\% Number of different pile driving playback files recorded:

pvector $=1: 3$; $\frac{\circ}{\circ}$ Chapter 2 used three files

\% pvector $=1$; $\%$ Chapters 3, 4, and Appendix E used one file

for $\mathrm{p}=$ pvector

sampvec = svec_cell\{p\}; \% Vector of samples for pile file segment

Hdata $\{\mathrm{p}\}=\operatorname{HdataFilt}($ sampvec $(1)$ : sampvec(end)); \% Segment hydrophone data

Adata $\{\mathrm{p}\}=$ AdataFull (sampvec(1): sampvec(end)); $\frac{\circ}{0}$ Segment accel, data

AXdata $\{\mathrm{p}\}=\operatorname{AXfilt}(\operatorname{sampvec}(1): \operatorname{sampvec}($ end $)) ;$

AYdata $\{\mathrm{p}\}=\operatorname{AYfilt}(\operatorname{sampvec}(1)$ : $\operatorname{sampvec}($ end $)) ;$

AZdata $\{\mathrm{p}\}=\operatorname{Azfilt}(\operatorname{sampvec}(1)$ : $\operatorname{sampvec}(e n d))$;

AXdata_nofilt $\{\mathrm{p}\}=$ XdataCal ( $\operatorname{sampvec}(1)$ : sampvec(end));

AYdata_nofilt $\{\mathrm{p}\}=$ YdataCal (sampvec(1) : $\operatorname{sampvec}($ end)) ;

AZdata_nofilt $\{\mathrm{p}\}=$ ZdataCal ( $\operatorname{sampvec}(1)$ : $\operatorname{sampvec}($ end)) ;

HdataForpks $=\operatorname{Hdata}\{\mathrm{p}\}$;

AdataForpks = Adata $\{\mathrm{p}\}$; 


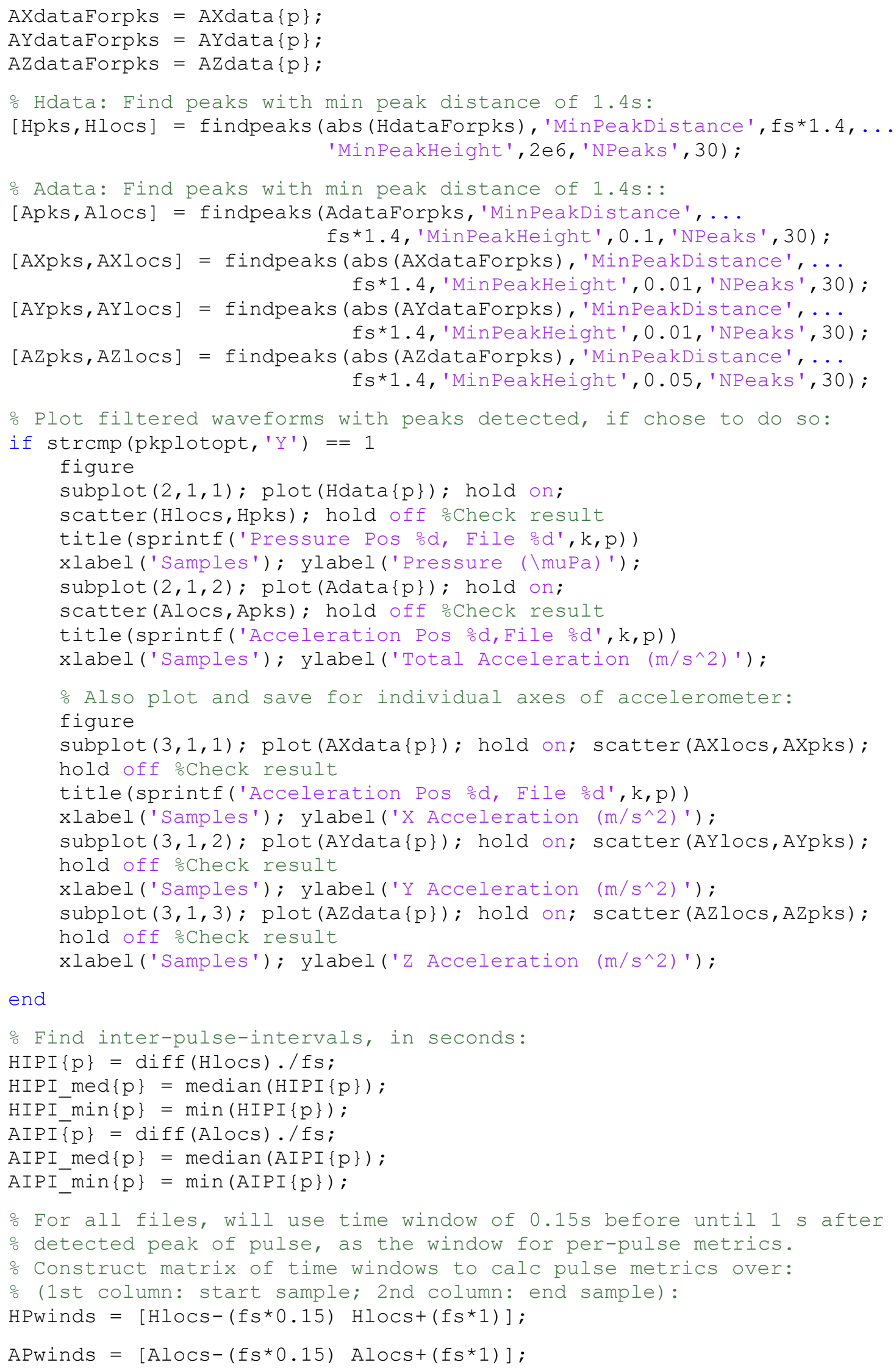




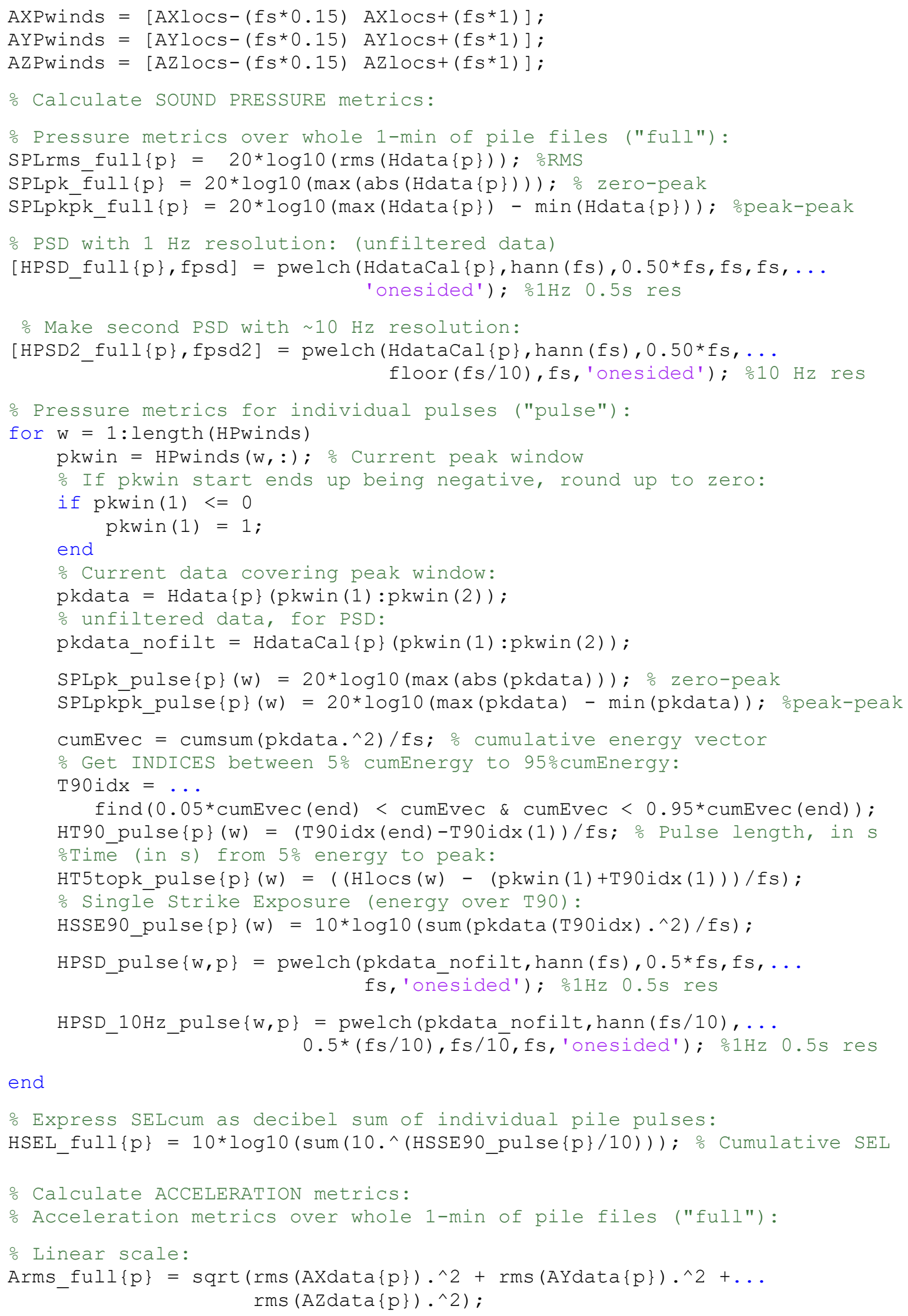




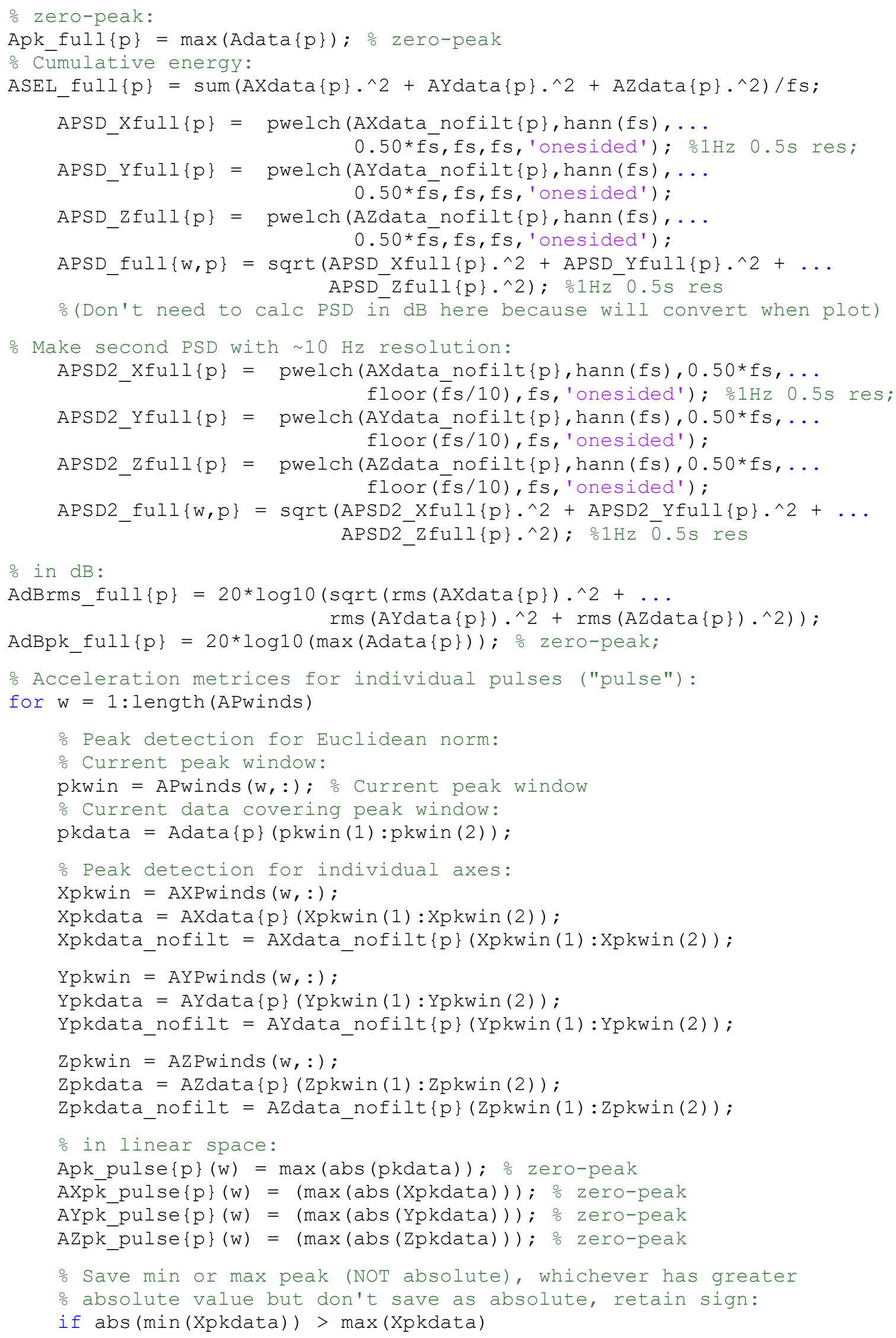




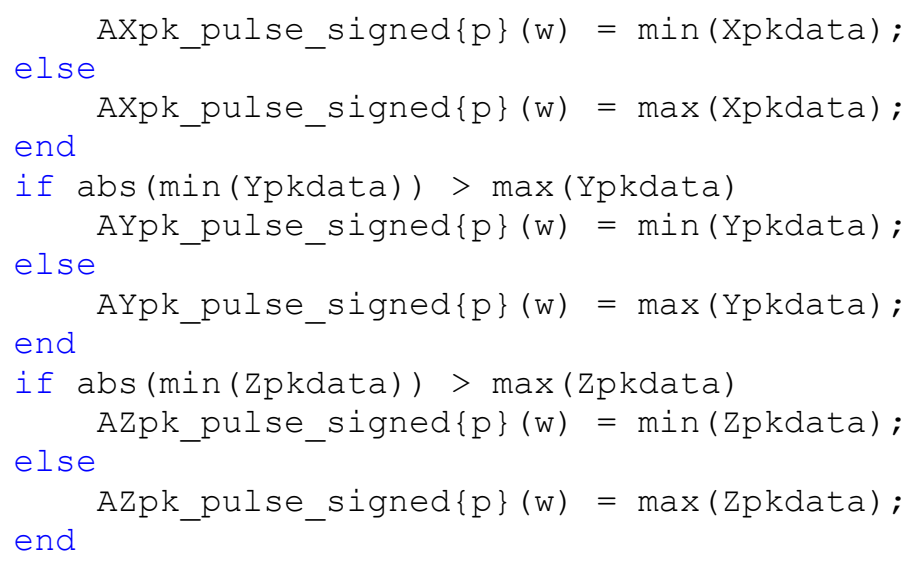


\%(Don't need to calc PSD in dB here because will convert when plot)

end

Express SELcum as decibel sum of individual pile pulses:

AdBSEL_full $\{\mathrm{p}\}=10 * \log 10\left(\operatorname{sum}\left(10 . \wedge\left(\operatorname{AdBSSE90\_ pulse}\{\mathrm{p}\} / 10\right)\right)\right)$;

end

end

\section{F2 MATLAB code used for Chapter 5 for calculating sound pressure, and particle acceleration using the finite-difference approximation}

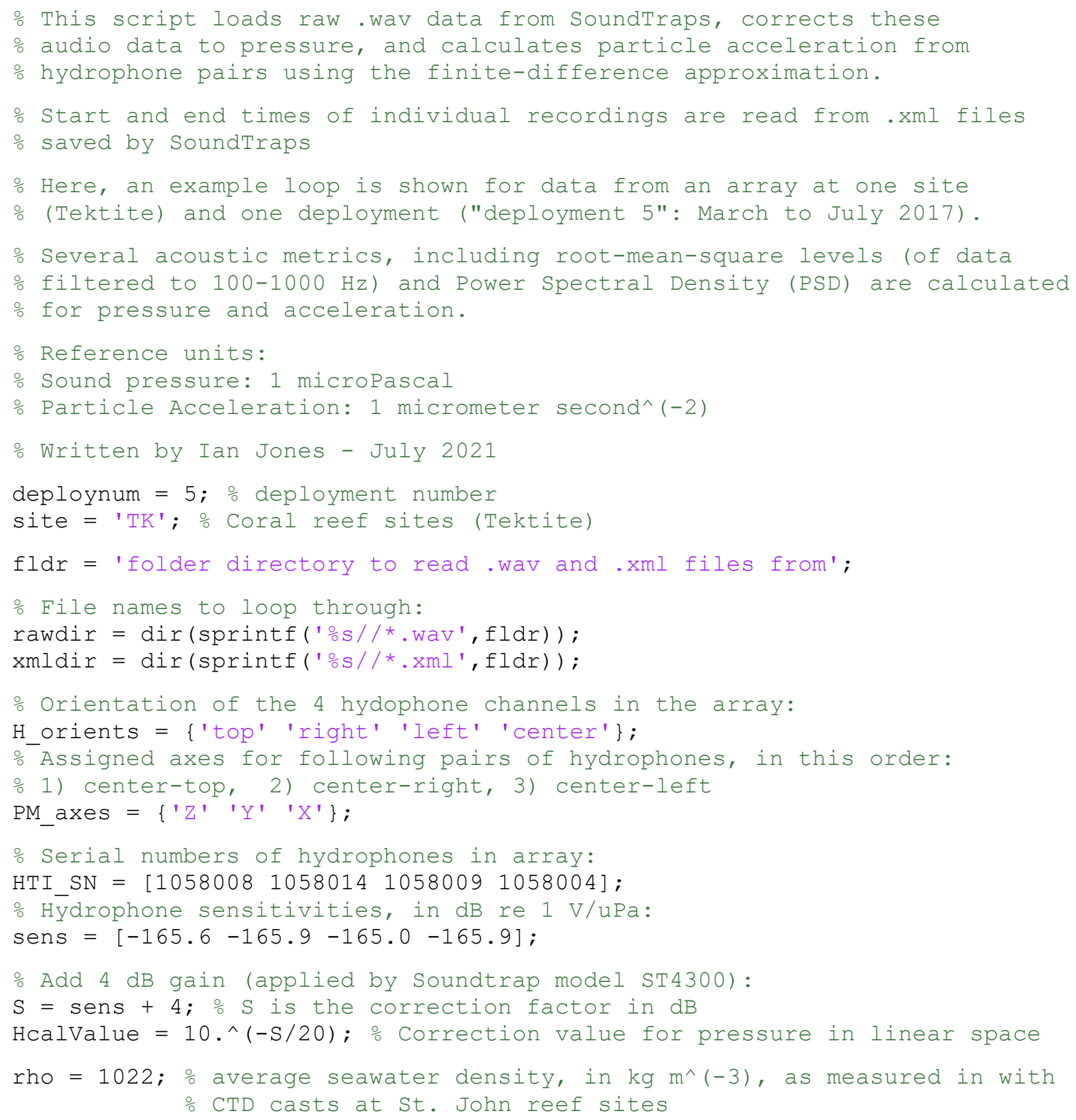




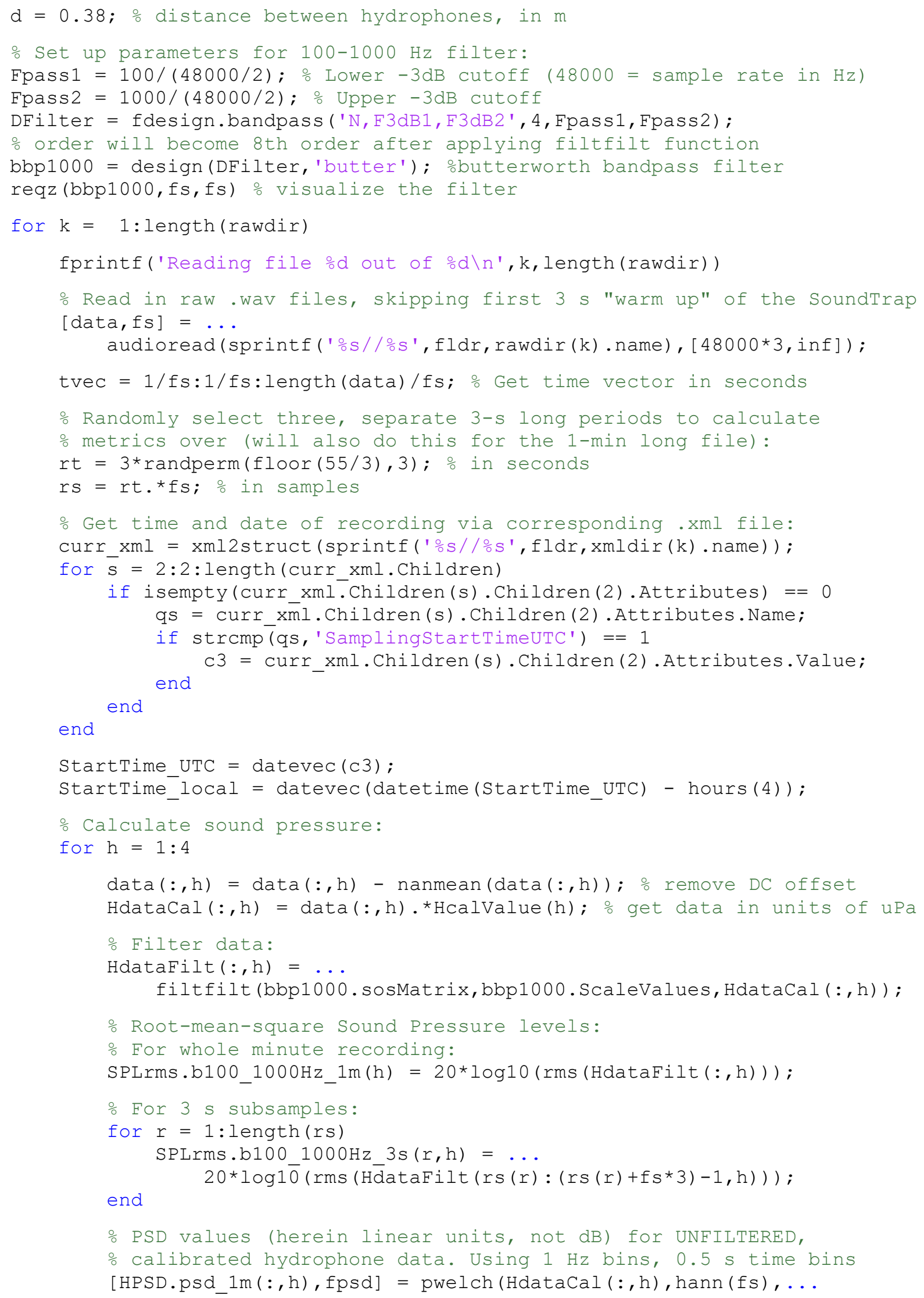




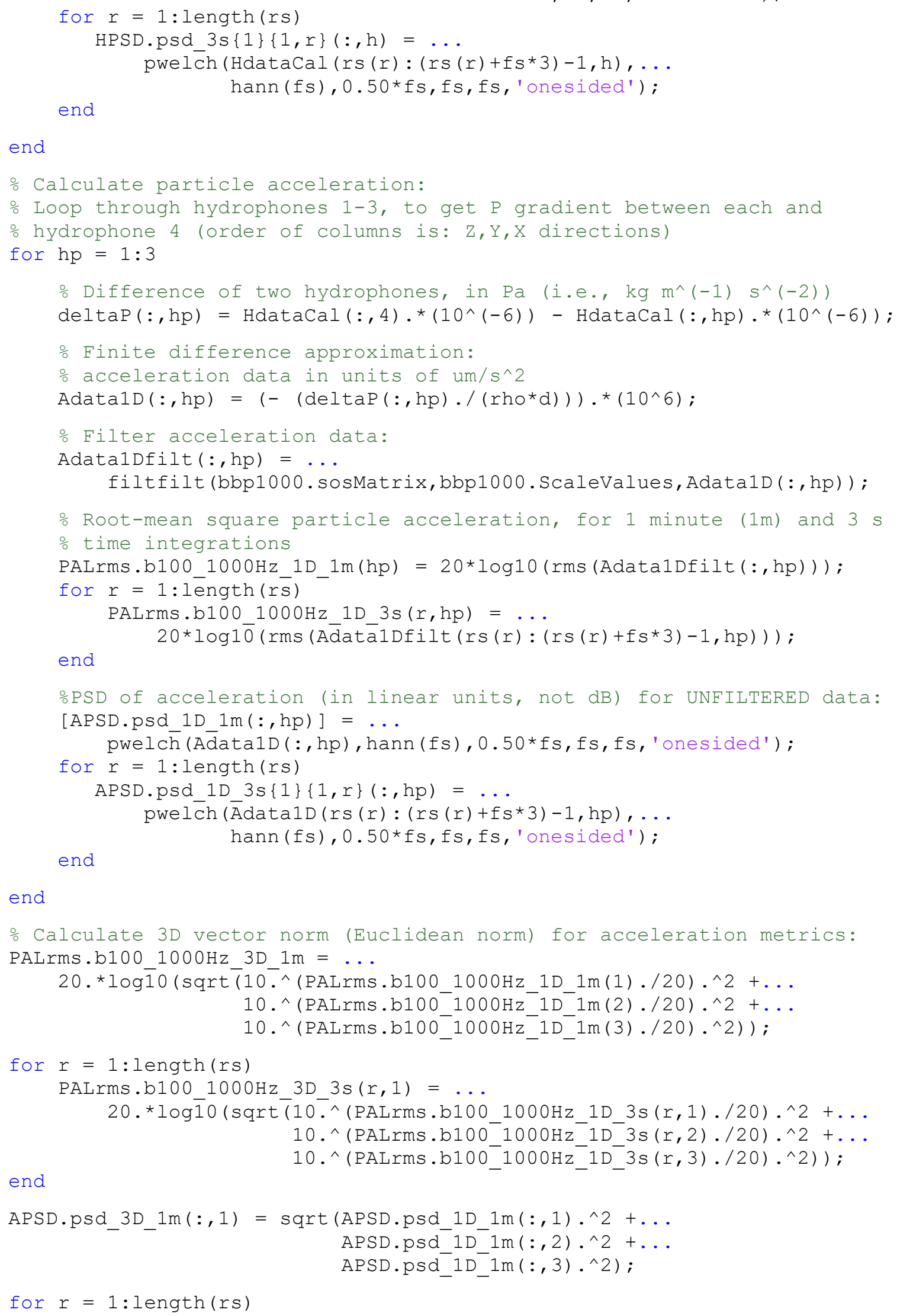




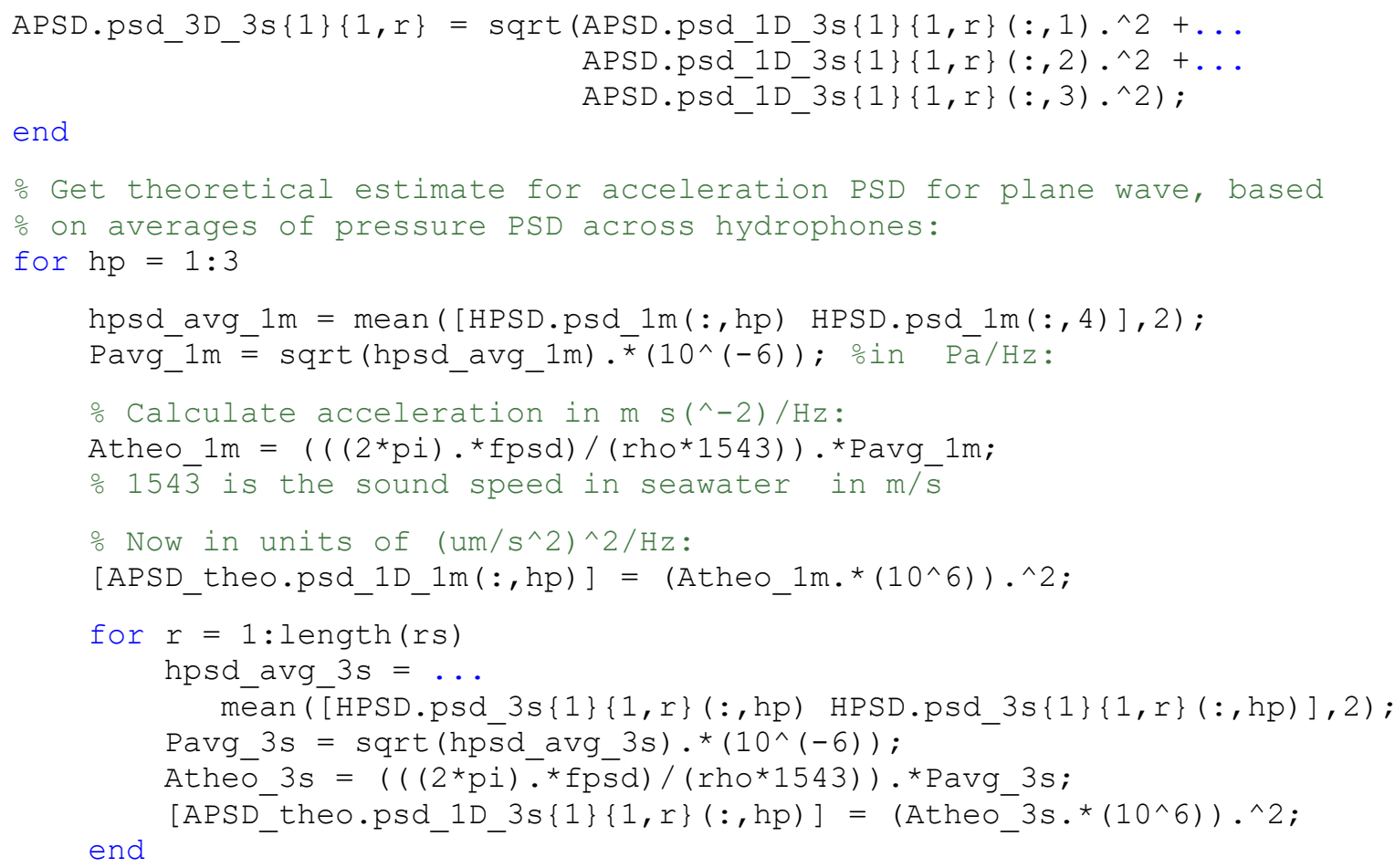

end

\% Make figure showing pressure waveform, PSD, and spectrogram from

\% just top hydrophone to use for auditing out boat files:

subplot $(3,1,1)$

plot (tvec, HdataCal $(:, 1))$;

ylabel ('Pressure (\muPa)'); xlabel('Time (s)');

title (datestr(StartTime_UTC));

subplot $(3,1,2)$

plot (fpsd (1:1001), 10*log10(HPSD.psd_1m(1:1001,1)));

ylabel('PSD (dB re $\left.\left.1 \backslash \mathrm{muPa}^{\wedge} 2\right)^{\prime}\right)$; $x l a b e l(' F r e q u e n c y ~(H z) ')$;

subplot $(3,1,3)$

spectrogram (HdataCal (:, 1), hann (12000), 0.50*12000,1:1000,fs, 'yaxis')

colorbar('Position', [ $\left.\begin{array}{lllll}0.91 & 0.157 & 0.04 & 0.17\end{array}\right)$

end 


\section{BIBLIOGRAPHY}

Aarts, G., Brasseur, S., \& Kirkwood, R. (2018). Behavioural response of grey seals to pile-driving. Wageningen Marine Research (University \& Research centre), Den Helder. https://doi.org/doi.org/10.18174/466039

Ainslie, M. A. (2010). Propagation of underwater sound. In Principles of sonar performance (pp. 439-512). Berlin Heidelberg: Springer.

Ainslie, M. A., Halvorsen, M. B., Müller, R. A. J., \& Lippert, T. (2020). Application of damped cylindrical spreading to assess range to injury threshold for fishes from impact pile driving. The Journal of the Acoustical Society of America, 148(1), 108-121. https://doi.org/10.1121/10.0001443

Akamatsu, T., Okumura, T., Novarini, N., \& Yan, H. Y. (2002). Empirical refinements applicable to the recording of fish sounds in small tanks. The Journal of the Acoustical Society of America, 112(6), 3073-3082. https://doi.org/10.1121/1.1515799

Altieri, A. H., Harrison, S. B., Seemann, J., Collin, R., Diaz, R. J., \& Knowlton, N. (2017). Tropical dead zones and mass mortalities on coral reefs. Proceedings of the National Academy of Sciences of the United States of America, 114(14), 3660-3665. https://doi.org/10.1073/pnas.1621517114

Amaral, J. L., Beard, R., Barham, R. J., Collett, A. G., Elliot, J., Frankel, A. S., Gallien, D., Hager, C., Khan, A. A., Lin, Y.-T., Mason, T., Miller, J. H., Newhall, A. E., Potty, G. R., Smith, K., \& Vigness-Raposa, K. (2018). Field observations during wind turbine foundation installation at the Block Island Wind Farm, Rhode Island, Appendix D: underwater sound monitoring reports. Retrieved from https://www.boem.gov/environment/field-observations-duringwind-turbine-foundation-installation-block-island-wind-farm

Amaral, J. L., Miller, J. H., Potty, G. R., Vigness-Raposa, K. J., Frankel, A. S., Lin, Y.-T., Newhall, A. E., Wilkes, D. R., \& Gavrilov, A. N. (2020). Characterization of impact pile driving signals during installation of offshore wind turbine foundations. Journal of the Acoustical Society of America, 147, 2323-2333. https://doi.org/10.1121/10.0001035

Amorim, M. C. P. (2006). Diversity of sound production in fish. In F. Ladich, S. P. Collin, P. Moller, \& P. G. Kapoor (Eds.), Communication in Fishes (pp. 71-105). Science Publishers.

Amoser, S., \& Ladich, F. (2005). Are hearing sensitivities of freshwater fish adapted to the ambient noise in their habitats? Journal of Experimental Biology, 208(18), 3533-3542. https://doi.org/10.1242/jeb.01809

Andrew, R. K., Howe, B. M., \& Mercer, J. A. (2011). Long-time trends in ship traffic noise for four sites off the North American West Coast. The Journal of the Acoustical Society of America, 129(2). https://doi.org/10.1121/1.3518770

Au, W. W. L., \& Banks, K. (1998). The acoustics of the snapping shrimp Synalpheus parneomeris in Kaneohe Bay. The Journal of the Acoustical Society of America, 103(1), 41-47. https://doi.org/10.1121/1.423234

Au, W. W. L., \& Hastings, M. C. (2008). Principles of Marine Bioacoustics. New York: Springer Science+Business Media.

Bailey, H., Brookes, K. L., \& Thompson, P. M. (2014). Assessing environmental impacts of offshore wind farms: lessons learned and recommendations for the future. Aquatic Biosystems, 10(8). https://doi.org/10.1186/2046-9063-10-8 
Bailey, H., Senior, B., Simmons, D., Rusin, J., Picken, G., \& Thompson, P. M. (2010). Assessing underwater noise levels during pile-driving at an offshore windfarm and its potential effects on marine mammals. Marine Pollution Bulletin, 60(6), 888-897. https://doi.org/10.1016/j.marpolbul.2010.01.003

Bates, D., Maechler, M., Bolker, B., \& Walker, S. (2015). Fitting linear mixed-effects models using Ime4. Journal of Statistical Software, 67(1), 1-48. https://doi.org/10.18637/jss.v067.i01

Bejder, L., Samuels, A., Whitehead, H., Finn, H., \& Allen, S. (2009). Impact assessment research: use and misuse of habituation, sensitisation and tolerance in describing wildlife responses to anthropogenic stimuli. Marine Ecology Progress Series, 395, 177-185. https://doi.org/10.3354/meps07979

Bellmann, M. A. (2014). Overview of existing noise mitigation systems for reducing pile-driving noise. INTERNOISE 2014 - 43rd International Congress on Noise Control Engineering: Improving the World Through Noise Control, 1-11.

Bellmann, M. A., Brinkmann, J., May, A., Wendt, T., Gerlach, S., \& Remmers, P. (2020). Underwater noise during percussive pile driving: Influencing factors on pile-driving noise and technical possibilities to comply with noise mitigation values. Federal Ministry for the Environment, Nature Conservation and Nuclear Safety, Federal Maritime and Hydrographic Agency. Retrieved from https://www.itap.de/media/experience_report_underwater_era-report.pdf

Bidder, A. M. (1950). The digestive mechanism of the European squids Logio vulgaris, Loligo forbesii, Alloteuthis media, and Alloteuthis subulata. The Quarterly Journal of Microscopical Science, 91(1), 1-44. Retrieved from http://www.ncbi.nlm.nih.gov/pubmed/24537669

Bittencourt, L., Barbosa, M., Bisi, T. L., Lailson-Brito, J., \& Azevedo, A. F. (2020). Anthropogenic noise influences on marine soundscape variability across coastal areas. Marine Pollution Bulletin, 160(September), 111648. https://doi.org/10.1016/j.marpolbul.2020.111648

Blom, E., Kvarnemo, C., Dekhla, I., Schöld, S., Andersson, M. H., Svensson, O., \& Amorim, M. C. P. (2019). Continuous but not intermittent noise has a negative impact on mating success in a marine fish with paternal care. Scientific Reports, 9(5494), 1-9. https://doi.org/10.1038/s41598-019-41786-x

BOEM. (2021). BOEM renewably energy lease and grant information. Retrieved March 25, 2021, from https://www.boem.gov/renewable-energy/lease-and-grant-information

Boyle, P. R., \& Rodhouse, P. (2005). Cephalopods: Ecology and fisheries. Ames, lowa: Blackwell Science.

Brodziak, J. K. T., \& Macy, W. K. (1996). Growth of long-finned squid, Loligo pealei, in the northwest Atlantic. Fishery Bulletin, 94, 212-236.

Brooks, M. E., Kristensen, K., van Benthem, K. J., Magnusson, A., Berg, C. W., Nielsen, A., Skaug, H. J., Maechler, M., \& Bolker, B. M. (2017). glmmTMB balances speed and flexibility among packages for zero-inflated generalized linear mixed modeling. The $R$ Journal, 9(2), 378400. Retrieved from https://journal.r-project.org/archive/2017/RJ-2017-066/index.html

Bruintjes, R., Purser, J., Everley, K. A., Mangan, S., Simpson, S. D., \& Radford, A. N. (2016). Rapid recovery following short-term acoustic disturbance in two fish species. Royal Society Open Science, 3(1), 150686. https://doi.org/10.1098/rsos.150686

Bruintjes, R., \& Radford, A. N. (2013). Context-dependent impacts of anthropogenic noise on 
individual and social behaviour in a cooperatively breeding fish. Animal Behaviour, 85(6), 1343-1349. https://doi.org/10.1016/j.anbehav.2013.03.025

Bruintjes, R., Simpson, S. D., Harding, H., Bunce, T., Benson, T., Rossington, K., \& Jones, D. (2017). The impact of experimental impact pile driving on oxygen uptake in black seabream and plaice. In Proceedings of Meetings on Acoustics (Vol. 27). https://doi.org/10.1121/2.0000422

Budelmann, B. U. (1979). Hair cell polarization in the gravity receptor systems of the statocysts of the cephalopods Sepia officinalis and Loligo vulgaris. Brain Research, 160(2), 261-270. https://doi.org/10.1016/0006-8993(79)90423-2

Budelmann, B. U. (1990). The statocysts of squid. In D. L. Gilbert, W. J. Adelman, \& J. M. Arnold (Eds.), Squid as Experimental Animals (pp. 421-442). New York: Plenum Press.

Budelmann, B. U. (1992a). Hearing in crustacea. In Douglas B Webster, R. R. Fay, \& A. N. Popper (Eds.), The Evolutionary Biology of Hearing (1st ed., pp. 131-140). New York: SpringerVerlag.

Budelmann, B. U. (1992b). Hearing in nonarthropod invertebrates. In D B Webster, R. R. Fay, \& A. N. Popper (Eds.), The Evolutionary Biology of Hearing (1st ed., pp. 141-155). New York: Springer-Verlag.

Budelmann, B. U., \& Bleckmann, H. (1988). A lateral line analogue in cephalopods: water waves generate microphonic potentials in the epidermal head lines of Sepia and Lolliguncula. Journal of Comparative Physiology A, 164(1), 1-5. https://doi.org/10.1007/BF00612711

Budelmann, B. U., Riese, U., \& Bleckmann, H. (1991). Structure, function, biological significance of the cuttlefish "lateral lines." The Cuttlefish, (1983), 201-209.

Budelmann, B. U., \& Tu, Y. (1997). The statocyst-oculomotor reflex of cephalopods and the vestibulo-oculomotor reflex of vertebrates: a tabular comparison. Vie et Milieu, 47(2), 9599.

Budelmann, B. U., \& Williamson, R. (1994). Directional sensitivity of hair cell afferents in the Octopus statocyst. The Journal of Experimental Biology, 187, 245-259. Retrieved from http://www.ncbi.nlm.nih.gov/pubmed/7931033

Buresch, K. C., Boal, J. G., Knowles, J., Debose, J., Nichols, A., Erwin, A., Painter, S. D., Nagle, G. T., \& Hanlon, R. T. (2003). Contact chemosensory cues in egg bundles elicit male-male agonistic conflicts in the squid Loligo pealeii. Journal of Chemical Ecology, 29(3), 547-560. https://doi.org/10.1023/A:1022846603591

Buresch, K. C., Boal, J. G., Nagle, G. T., Knowles, J., Nobuhara, R., Sweeney, K., \& Hanlon, R. T. (2004). Experimental evidence that ovary and oviducal gland extracts influence male agonistic behavior in squids. Biological Bulletin, 206(1), 1-3. https://doi.org/10.2307/1543192

Buscaino, G., Gavio, A., Galvan, D., Filiciotto, F., Maccarrone, V., de Vincenzi, G., Mazzola, S., \& Orensanz, J. M. (2015). Acoustic signals and behaviour of Ovalipes trimaculatus in the context of reproduction. Aquatic Biology, 24(1), 61-73. https://doi.org/10.3354/ab00636

Butler, J., Pagniello, C. M. L. S., Jaffe, J. S., Parnell, P. E., \& Širović, A. (2021). Diel and Seasonal Variability in Kelp Forest Soundscapes Off the Southern California Coast. Frontiers in Marine Science, 8(April), 1-14. https://doi.org/10.3389/fmars.2021.629643

Caiger, P. E., Montgomery, J. C., \& Radford, C. A. (2012). Chronic low-intensity noise exposure affects the hearing thresholds of juvenile snapper, 466, 225-232. 
https://doi.org/10.3354/meps09933

Campbell, J., Sabet, S. S., \& Slabbekoorn, H. (2019). Particle motion and sound pressure in fish tanks: a behavioural exploration of acoustic sensitivity in the zebrafish. Behavioural Processes, 164, 38-47.

Carey, D. A., Wilber, D. H., Read, L. B., Guarinello, M. L., Griffin, M., \& Sabo, S. (2020). Effects of the Block Island Wind Farm on coastal resources. Oceanography, 33(4), 70-81. Retrieved from https://www.jstor.org/stable/10.2307/26965751

Carroll, A. G., Przeslawski, R., Duncan, A., Gunning, M., \& Bruce, B. (2017). A critical review of the potential impacts of marine seismic surveys on fish $\&$ invertebrates. Marine Pollution Bulletin, 114(1), 9-24. https://doi.org/10.1016/j.marpolbul.2016.11.038

Casper, B. M., Halvorsen, M. B., Matthews, F., Carlson, T. J., \& Popper, A. N. (2013). Recovery of barotrauma injuries resulting from exposure to pile driving sound in two sizes of hybrid striped bass. PLOS ONE, 8(9), e73844. https://doi.org/10.1371/journal.pone.0073844

Casper, B. M., \& Mann, D. A. (2007). The directional hearing abilities of two species of bamboo sharks. The Journal of Experimental Biology, 210(3), 505-511. https://doi.org/10.1242/jeb.02677

Casper, B. M., Smith, M. E., Halvorsen, M. B., Sun, H., Carlson, T. J., \& Popper, A. N. (2013). Effects of exposure to pile driving sounds on fish inner ear tissues. Comparative Biochemistry and Physiology, Part A, 166(2), 352-360. https://doi.org/10.1016/j.cbpa.2013.07.008

Celi, M., Filiciotto, F., Vazzana, M., Arizza, V., Maccarrone, V., Ceraulo, M., Mazzola, S., \& Buscaino, G. (2015). Shipping noise affecting immune responses of European spiny lobster (Palinurus elephas). Canadian Journal of Zoology, 121(3), 113-121. https://doi.org/10.1139/cjz-2014-0219

Ceraulo, M., Bruintjes, R., Benson, T., Rossington, K., Farina, A., \& Buscaino, G. (2016). Relationships of sound pressure and particle velocity during pile driving in a flooded dock. Proceedings of Meetings on Acoustics, 27(040007). https://doi.org/10.1121/2.0000295

Chan, A. A. Y., \& Blumstein, D. T. (2011). Attention, noise, and implications for wildlife conservation and management. Applied Animal Behaviour Science, 131, 1-7.

Chan, A. A. Y., Giraldo-perez, P., Smith, S., \& Blumstein, D. T. (2010). Anthropogenic noise affects risk assessment and attention: the distracted prey hypothesis. Biology Letters, 6, 458-461. https://doi.org/10.1098/rsbl.2009.1081

Charifi, M., Sow, M., Ciret, P., Benomar, S., \& Massabuau, C. (2017). The sense of hearing in the Pacific oyster, Magallana gigas. PLoS ONE, 12(10), e0185353. https://doi.org/10.1371/journal.pone.0185353 October

Clark, C. W., Ellison, W. T., Southall, B. L., Hatch, L., Parijs, S. M. Van, Frankel, A., \& Ponirakis, D. (2009). Acoustic masking in marine ecosystems: intuitions, analysis, and implication. Marine Ecology Progress Series, 395, 201-222. https://doi.org/10.3354/meps08402

Codarin, A., Wysocki, L. E., Ladich, F., \& Picciulin, M. (2009). Effects of ambient and boat noise on hearing and communication in three fish species living in a marine protected area (Miramare, Italy). Marine Pollution Bulletin, 58(12), 1880-1887. https://doi.org/10.1016/j.marpolbul.2009.07.011

Coombs, S., \& Braun, C. B. (2003). Information processing by the lateral line system. In Shaun P Collin \& J. N. Marshall (Eds.), Sensory Processing in Aquatic Environments (pp. 122-138). 
New York: Springer.

Costello, M. J., Coll, M., Danovaro, R., Halpin, P., Ojaveer, H., \& Miloslavich, P. (2010). A census of marine biodiversity knowledge, resources, and future challenges. PLOS ONE, 5(8). https://doi.org/10.1371/journal.pone.0012110

Crook, R. J., Dickson, K., Hanlon, R. T., \& Walters, E. T. (2014). Nociceptive sensitization reduces predation risk. Current Biology, 24(10), 1121-1125. https://doi.org/10.1016/j.cub.2014.03.043

Cummins, S. F., Boal, J. G., Buresch, K. C., Kaunpradit, C., Sobhorn, P., Holm, J. B., Degnan, B. M., Nagle, G. T., \& Hanlon, R. T. (2011). Extreme aggression in male squid induced by a betaMSP-like pheromone. Current Biology, 21, 322-327. https://doi.org/10.1016/j.cub.2011.01.038

Dahl, P., de Jong, C., \& Popper, A. (2015). The underwater sound field from impact pile driving and its potential effects on marine life. Acoustics Today, 11(2). Retrieved from http://acousticstoday.org/wp-content/uploads/2015/06/The-Underwater-Sound-Fieldfrom-Impact-Pile-Driving-and-Its-Potential-Effects-on-Marine-Life-Peter-H.-Dahl-Christ-A.F.-de-Jong-and-Arthur-N.-Popper.pdf

Dähne, M., Tougaard, J., Carstensen, J., Rose, A., \& Nabe-nielsen, J. (2017). Bubble curtains attenuate noise from offshore wind farm construction and reduce temporary habitat loss for harbour porpoises. Marine Ecology Progress Series, 580, 221-237. https://doi.org/doi.org/10.3354/meps12257

Dale, J. J., Gray, M. D., Popper, A. N., Rogers, P. H., \& Block, B. A. (2015). Hearing thresholds of swimming Pacific bluefin tuna Thunnus orientalis. Journal of Comparative Physiology A: Neuroethology, Sensory, Neural, and Behavioral Physiology, 201(5), 441-454. https://doi.org/10.1007/s00359-015-0991-x

Day, R. D., Mccauley, R. D., Fitzgibbon, Q. P., Hartmann, K., \& Semmens, J. M. (2019). Seismic air guns damage rock lobster mechanosensory organs and impair righting reflex. Proceedings of the National Academy of Sciences of the United States of America, 286, 20191424. https://doi.org/10.1098/rspb.2019.1424

Day, R. D., McCauley, R. D., Fitzgibbon, Q. P., Hartmann, K., \& Semmens, J. M. (2017). Exposure to seismic air gun signals causes physiological harm and alters behavior in the scallop Pecten fumatus. Proceedings of the National Academy of Sciences. https://doi.org/10.1073/pnas.1700564114

de Jong, K., Forland, T. N., Amorim, M. C. P., Rieucau, G., Slabbekoorn, H., \& Sivle, L. D. (2020). Predicting the effects of anthropogenic noise on fish reproduction. Reviews in Fish Biology and Fisheries, 30, 245-268. https://doi.org/10.1007/s11160-020-09598-9

de Soto, N. A., Delorme, N., Atkins, J., Howard, S., Williams, J., \& Johnson, M. (2013). Anthropogenic noise causes body malformations and delays development in marine larvae. Scientific Reports, 3, 2831. https://doi.org/10.1038/srep02831

Debusschere, E., De Coensel, B., Bajek, A., Botteldooren, D., Hostens, K., Vanaverbeke, J., Vandendriessche, S., Van Ginderdeuren, K., Vincx, M., \& Degraer, S. (2014). In situ mortality experiments with juvenile sea bass (Dicentrarchus labrax) in relation to impulsive sound levels caused by pile driving of windmill foundations. PLOS ONE, 9(10), 1-9. https://doi.org/10.1371/journal.pone.0109280

Derby, C. D., Tottempudi, M., Love-Chezem, T., \& Wolfe, L. S. (2013). Ink from longfin inshore 
squid, Doryteuthis pealeii, as a chemical and visual defense against two predatory fishes, summer flounder, Paralichthys dentatus, and sea catfish, Ariopsis felis. Biological Bulletin, 225(December), 152-160.

Dinh, J. P., Suca, J. J., Lillis, A., Apprill, A., Llopiz, J. K., \& Mooney, T. A. (2018). Multiscale spatiotemporal patterns of boat noise on U.S. Virgin Island coral reefs. Marine Pollution Bulletin, 136, 282-290. https://doi.org/10.1016/j.marpolbul.2018.09.009

DNH. (n.d.). Aqua-30. Retrieved from http://www.dnh.no/speakers/aqua-30-365.aspx

Dukas, R. (2002). Behavioural and ecological consequences of limited attention. Philosophical Transactions of the Royal Society London B: Biological Sciences, 357, 1539-1547. https://doi.org/10.1098/rstb.2002.1063

Edmunds, P. J. (2013). Decadal-scale changes in the community structure of coral reefs of St. John, US Virgin Islands. Marine Ecology Progress Series, 489, 107-123. https://doi.org/10.3354/meps10424

Edmunds, P. J., Coblentz, M., \& Wulff, J. (2020). A quarter-century of variation in sponge abundance and community structure on shallow reefs in St. John, US Virgin Islands. Marine Biology, 167(135). https://doi.org/10.1007/s00227-020-03740-8

Eichstaedt, K. E., Kovatch, K., \& Aaron, D. (2013). A less conservative method to adjust for familywise error rate in neuropsychological research: the Holm's sequential Bonferroni procedure. NeuroRehabilitation, 32, 693-696. https://doi.org/10.3233/NRE-130893

Elise, S., Urbina-Barreto, I., Pinel, R., Mahamadaly, V., Bureau, S., Penin, L., Adjeroud, M., Kulbicki, M., \& Bruggemann, J. H. (2019). Assessing key ecosystem functions through soundscapes: a new perspective from coral reefs. Ecological Indicators, 107, 105623. https://doi.org/10.1016/j.ecolind.2019.105623

Ellison, W. T., Southall, B. L., Clark, C. W., \& Frankel, A. S. (2012). A new context-based approach to assess marine mammal behavioral responses to anthropogenic sounds. Conservation Biology, 26(1), 21-28. https://doi.org/10.1111/j.1523-1739.2011.01803.x

Erbe, C., Marley, S. A., Schoeman, R. P., Smith, J. N., Trigg, L. E., \& Embling, C. B. (2019). The effects of ship noise on marine mammals - a review. Frontiers in Marine Science, 6. https://doi.org/10.3389/fmars.2019.00606

Fabricius, K. E. (2005). Effects of terrestrial runoff on the ecology of corals and coral reefs: review and synthesis. Marine Pollution Bulletin, 50(2), 125-146. https://doi.org/10.1016/j.marpolbul.2004.11.028

FAO. (2021). FAO fisheries and aquaculture statistics. Retrieved October 6, 2021, from http://www.fao.org/fishery/statistics/en

Farina, A., \& Gage, S. H. (2017). Ecoacoustics. Oxford: John Wiley \& Sons.

Faure, P. A., Mason, A. C., \& Yack, J. E. (2008). Invertebrate ears and hearing. Encyclopedia of Neuroscience, 2035-2042. https://doi.org/10.1007/978-3-540-29678-2_2580

Fay, R. (2009). Soundscapes and the sense of hearing of fishes. Integrative Zoology, 4(1), 26-32. https://doi.org/10.1111/j.1749-4877.2008.00132.x

Fay, R., \& Passow, B. (1982). Temporal discrimination in the goldfish. Journal of the Acoustical Society of America, 72(3), 753-760. https://doi.org/10.1121/1.388255

Ferrier-Pagès, C., Leal, M. C., Calado, R., Schmid, D. W., Bertucci, F., Lecchini, D., \& Allemand, D. (2021). Noise pollution on coral reefs? - A yet underestimated threat to coral reef communities. Marine Pollution Bulletin, 165(February). 
https://doi.org/10.1016/j.marpolbul.2021.112129

Fewtrell, J. L., \& McCauley, R. D. (2012). Impact of air gun noise on the behaviour of marine fish and squid. Marine Pollution Bulletin, 64(5), 984-993.

https://doi.org/10.1016/j.marpolbul.2012.02.009

Filiciotto, F., Paz, M., Moyano, S., Vincenzi, G. De, Hidalgo, F., Sciacca, V., Cielo, M., Corrias, V., Lorusso, M., Mazzola, S., Buscaino, G., \& Andrea, M. (2018). Are semi-terrestrial crabs threatened by human noise? Assessment of behavioural and biochemical responses of Neohelice granulata (Brachyura, Varunidae) in tank. Marine Pollution Bulletin, 137, 24-34. https://doi.org/10.1016/j.marpolbul.2018.07.023

Filiciotto, F., Vazzana, M., Celi, M., Maccarrone, V., Ceraulo, M., Buffa, G., Arizza, V., Vincenzi, G. de, Grammauta, R., Mazzola, S., \& Buscaino, G. (2016). Underwater noise from boats: measurement of its influence on the behaviour and biochemistry of the common prawn (Palaemon serratus, Pennant 1777). Journal of Experimental Marine Biology and Ecology, 478, 24-33. https://doi.org/10.1016/j.jembe.2016.01.014

Fox, J., \& Weisberg, S. (2019). An $\{R\}$ companion to applied regression (Third). Thousand Oaks CA: Sage. Retrieved from https://socialsciences.mcmaster.ca/jfox/Books/Companion/

Freeman, L., \& Freeman, S. (2016). Rapidly obtained ecosystem indicators from coral reef soundscapes. Marine Ecology Progress Series, 561, 69-82. https://doi.org/10.3354/meps11938

Frisk, G. V. (2012). Noiseonomics: the relationship between ambient noise levels in the sea and global economic trends. Scientific Reports, 2(437). https://doi.org/10.1038/srep00437

Gedamke, J., Harrison, J., Hatch, L., Angliss, R., Barlow, J., Berchok, C., Caldow, C., Castellote, M., Cholewiak, D., Deangelis, M. L., Dziak, R., Garland, E., Guan, S., Hastings, S., Holt, M., Laws, B., Mellinger, D., Moore, S., Moore, T. J., et al. (2016). Ocean noise strategy roadmap. Retrieved from https://cetsound.noaa.gov/road-map

Gilbert, Daniel L, Adelman, W. J., \& Arnold, J. M. (1990). Squid as Experimental Animals. (Daniel L Gilbert, W. J. Adelman, \& J. M. Arnold, Eds.) (1st ed.). New York: Springer Science+Business Media.

Gleadall, I. G., \& Shashar, N. (2004). The octopus's garden: the visual world of cephalopods. In F. R. Prete (Ed.), Complex Worlds from Simpler Nervous Systems (pp. 269-308). Cambridge, MA: MIT Press.

Gordon, T. A. C., Harding, H. R., Wong, K. E., Merchant, N. D., Meekan, M. G., Mccormick, M. I., Radford, A. N., \& Simpson, S. D. (2018). Habitat degradation negatively affects auditory settlement behavior of coral reef fishes, 1-6. https://doi.org/10.1073/pnas.1719291115

Gordon, T. A. C., Radford, A. N., Davidson, I. K., Barnes, K., McCloskey, K., Nedelec, S. L., Meekan, M. G., McCormick, M. I., \& Simpson, S. D. (2019). Acoustic enrichment can enhance fish community development on degraded coral reef habitat. Nature Communications, 10(1), 1-7. https://doi.org/10.1038/s41467-019-13186-2

Graham, I. M., Merchant, N. D., Farcas, A., Barton, T. R., Cheney, B., Bono, S., \& Thompson, P. M. (2019). Harbour porpoise responses to pile-driving diminish over time. Royal Society Open Science, 6, 190335. https://doi.org/10.1098/rsos.190335

Gray, M. D., Rogers, P. H., \& Zeddies, D. G. (2016). Acoustic particle motion measurement for bioacousticians: principles and pitfalls. Proceedings of Meetings on Acoustics, 27(1). https://doi.org/10.1121/2.0000290 
Halvorsen, M. B., Casper, B. M., Woodley, C. M., Carlson, T. J., \& Popper, A. N. (2011). Hydroacoustic impacts on fish from pile installation. Washington, D.C. https://doi.org/10.17226/14596

Hanlon, R. T. (1990). Maintenance, Rearing, and Culture of Teuthoid and Sepioid Squids. In D. Gilbert, W. Adelman, \& J. Arnold (Eds.), Squid as Experimental Animals (pp. 35-62). New York: Plenum Press.

Hanlon, R. T., \& Budelmann, B. U. (1987). Why cephalopods are probably not "deaf." The American Naturalist, 129(2), 312-317.

Hanlon, R. T., Buresch, K. C., Moustahfid, H., \& Staudinger, M. D. (2013). Doryteuthis pealeii, longfin inshore squid. In R. Rosa, R. O’Dor, \& G. J. Pierce (Eds.), Advances in squid biology, ecology and fisheries, part I (pp. 205-240). New York: Nova Science Publishers, Inc.

Hanlon, R. T., Claes, M. F., Ashcraft, S. E., \& Dunlap, P. V. (1997). Laboratory culture of the sepiolid squid Euprymna scolopes: a model system for bacteria-animal symbiosis. Biological Bulletin, 192(3), 364-374. https://doi.org/10.2307/1542746

Hanlon, R. T., Maxwell, M. R., Shashar, N., Loew, E. R., \& Boyle, K. L. (1999). An ethogram of body patterning behavior in the biomedically and commercially valuable squid Loligo pealeii off Cape Cod, Massachusetts. Biol. Bull., 197(1), 49-62. https://doi.org/10.2307/1542996

Hanlon, R. T., \& Messenger, J. B. (1988). Adaptive coloration in young cuttlefish (Sepia Officinalis L .): the morphology and development of body patterns and their relation to behaviour. Philosophical Transactions of the Royal Society London B: Biological Sciences, 320(1200), 437-487. Retrieved from http://www.jstor.org/stable/2396667

Hanlon, R. T., \& Messenger, J. B. (2018). Cephalopod Behaviour (2nd ed.). New York: Cambridge University Press.

Hanlon, R. T., \& Shashar, N. (2003). Aspects of the sensory ecology of cephalopods. In S P Collin \& N. J. Marshall (Eds.), Sensory Processing in Aquatic Environments (pp. 266-282). Heidelberg, Germany: Springer-Verlag.

Hara, T., \& Hara, R. (1976). Distribution of rhodopsin and retinochrome in the squid retina. The Journal of General Physiology, 67, 791-805.

Harris, C. M., Thomas, L., Falcone, E. A., Hildebrand, J., Houser, D., Kvadsheim, P. H., Lam, F. P. A., Miller, P. J. O., Moretti, D. J., Read, A. J., Slabbekoorn, H., Southall, B. L., Tyack, P. L., Wartzok, D., \& Janik, V. M. (2018). Marine mammals and sonar: dose-response studies, the risk-disturbance hypothesis and the role of exposure context. Journal of Applied Ecology, 55(1), 396-404. https://doi.org/10.1111/1365-2664.12955

Hatfield, E. M. C., Hanlon, R. T., Forsythe, J. W., \& Grist, E. P. M. (2001). Laboratory testing of a growth hypothesis for juvenile squid Loligo pealeii (Cephalopoda: Loliginidae). Canadian Journal of Fisheries and Aquatic Sciences, 58, 845-857. https://doi.org/10.1139/cjfas-58-5845

Haver, S. M., Klinck, H., Nieukirk, S. L., Matsumoto, H., Dziak, R. P., \& Miksis-Olds, J. L. (2017). The not-so-silent world: measuring Arctic, Equatorial, and Antarctic soundscapes in the Atlantic Ocean. Deep-Sea Research Part I, 122, 95-104. https://doi.org/10.1016/j.dsr.2017.03.002

Hawkins, A. D., \& Chapman, C. J. (1975). Masked auditory thresholds in the cod Gadus morhua L. Journal of Comparative Physiology, 103, 209-226. 
Hawkins, A. D., Pembroke, A. E., \& Popper, A. N. (2015). Information gaps in understanding the effects of noise on fishes and invertebrates. Reviews in Fish Biology and Fisheries, 25(1), 39-64. https://doi.org/10.1007/s11160-014-9369-3

Hawkins, A. D., \& Popper, A. N. (2018). Directional hearing and sound source localization by fishes. The Journal of the Acoustical Society of America, 144(6), 3329-3350. https://doi.org/10.1121/1.5082306

Hawkins, A. D., Roberts, L., \& Cheesman, S. (2014). Responses of free-living coastal pelagic fish to impulsive sounds. The Journal of the Acoustical Society of America, 135(5), 3101-3116. https://doi.org/10.1121/1.4870697

Herbert-Read, J. E., Kremer, L., Bruintjes, R., Radford, A. N., \& loannou, C. C. (2017a). Anthropogenic noise pollution from pile-driving disrupts the structure and dynamics of fish shoals. Proceedings of the Royal Society B: Biological Sciences, 284. https://doi.org/10.1098/rspb.2017.1627

Herbert-Read, J. E., Kremer, L., Bruintjes, R., Radford, A. N., \& loannou, C. C. (2017b). Anthropogenic noise pollution from pile-driving disrupts the structure and dynamics of fish shoals. Proceedings of the Royal Society B: Biological Sciences, 284. https://doi.org/http://dx.doi.org/10.1098/rspb.2017.1627

Heres, M. M., Farmer, B. H., Elmer, F., \& Hertler, H. (2021). Ecological consequences of Stony Coral Tissue Loss Disease in the Turks and Caicos Islands. Coral Reefs, 40(2), 609-624. https://doi.org/10.1007/s00338-021-02071-4

Higgs, D. M., \& Radford, C. A. (2016). The potential overlapping roles of the ear and lateral line in driving "acoustic" responses. In J A Sisneros (Ed.), Fish Hearing and Bioacoustics (pp. 255-270). Springer International Publishing Switzerland. https://doi.org/10.1007/978-3319-21059-9

Holles, S. H., Simpson, S. D., Radford, A. N., Berten, L., \& Lecchini, D. (2013). Boat noise disrupts orientation behaviour n a coral reef fish. Marine Ecology Progress Series, 485, 295-300. https://doi.org/10.3354/meps10346

Horch, K., \& Salmon, M. (1973). Adaptations to the acoustic environment by the squirrelfishes Myripristis violaceus and M. Pralinius. Marine Behaviour and Physiology, 2(1-4), 121-139. https://doi.org/10.1080/10236247309386920

Horodysky, A. Z., Brill, R. W., Fine, M. L., Musick, J. A., \& Latour, R. J. (2008). Acoustic pressure and particle motion thresholds in six sciaenid fishes. Journal of Experimental Biology, 211(9), 1504-1511. https://doi.org/10.1242/jeb.016196

Hubert, J., Neo, Y. Y., Campbell, J. A., \& Slabbekoorn, H. (2016). Particle motion and pressure soundscape in outdoor vs indoor set-up. Proceedings of Meetings on Acoustics, 27(1). https://doi.org/10.1121/2.0000273

Hughes, A. R., Mann, D. A., \& Kimbro, D. L. (2014). Predatory fish sounds can alter crab foraging behaviour and influence bivalve abundance. Proceedings. Biological Sciences / The Royal Society, 281(1788), 20140715. https://doi.org/10.1098/rspb.2014.0715

Hughes, T. P., Anderson, K. D., Connolly, S. R., Heron, S. F., Kerry, J. T., Lough, J. M., Baird, A. H., Baum, J. K., Berumen, M. L., Bridge, T. C., Claar, D. C., Eakin, C. M., Gilmour, J. P., Graham, N. A. J., Harrison, H., Hobbs, J. P. A., Hoey, A. S., Hoogenboom, M., Lowe, R. J., et al. (2018). Spatial and temporal patterns of mass bleaching of corals in the Anthropocene. Science, 359(6371), 80-83. https://doi.org/10.1126/science.aan8048 
Hunsicker, M. E., \& Essington, T. E. (2006). Size-structured patterns of piscivory of the longfin inshore squid (Loligo pealeii) in the mid-Atlantic continental shelf ecosystem. Canadian Journal of Fisheries and Aquatic Sciences, 63(May 2017), 754-765. https://doi.org/10.1139/FO5-258

Hunsicker, M. E., Essington, T. E., Watson, R., \& Sumaila, U. R. (2010). The contribution of cephalopods to global marine fisheries: can we have our squid and eat them too? Fish and Fisheries, 11(4), 421-438. https://doi.org/10.1111/j.1467-2979.2010.00369.x

ISO/DIS. (2017). ISO 18405:2017 Underwater acoustics - Terminology. Geneva, Switzerland. Retrieved from https://www.iso.org/obp/ui/fr/\#iso:std:iso:18405:ed-1:v1:en:fn:1

Ivanova, S. V, Kessel, S. T., Espinoza, M., McLean, M. F., O’Neill, C., Landry, J., Hussey, N. E., Williams, R., Vagle, S., \& Fisk, A. T. (2020). Shipping alters the movement and behavior of Arctic cod (Boreogadus saida), a keystone fish in Arctic marine ecosystems. Ecological Applications, 0(0), 1-13. https://doi.org/10.1002/eap.2050

Jesus, S. M., Xavier, F. C., Vio, R. P., Osowsky, J., Simões, M. V. S., \& Netto, E. B. F. (2020). Particle motion measurements near a rocky shore off Cabo Frio Island. The Journal of the Acoustical Society of America, 147(6), 4009-4019. https://doi.org/10.1121/10.0001392 Jézéquel, Y., Bonnel, J., Coston-Guarini, J., \& Chauvaud, L. (2019). Revisiting the bioacoustics of European spiny lobsters Palinurus elephas: comparison of antennal rasps in tanks and in situ. Marine Ecology Progress Series, 615, 143-157. https://doi.org/10.3354/meps12935 Jézéquel, Y., Coston-Guarini, J., Chauvaud, L., \& Bonnel, J. (2020). Acoustic behaviour of male European lobsters (Homarus gammarus) during agonistic encounters. Journal of Experimental Biology, 223(4). https://doi.org/10.1242/jeb.211276

Jézéquel, Y., Jones, I. T., Bonnel, J., Chauvaud, L., Atema, J., \& Mooney, T. A. (2021). Sound detection by the American lobster (Homarus americanus). Journal of Experimental Biology, 224(6). https://doi.org/10.1242/jeb.240747

Jones, I. T., Peyla, J. F., Clark, H., Song, Z., Stanley, J. A., \& Mooney, T. A. (2021). Changes in feeding behavior of longfin squid (Doryteuthis pealeii) during laboratory exposure to pile driving noise. Marine Environmental Research, 165. https://doi.org/10.1016/j.marenvres.2020.105250

Jones, I. T., Stanley, J. A., Bonnel, J., \& Mooney, T. A. (2019). Complexities of tank acoustics warrant direct, careful measurement of particle motion and pressure for bioacoustic studies. Proceedings of Meetings on Acoustics, 37(010005). https://doi.org/10.1121/2.0001073

Jones, I. T., Stanley, J. A., \& Mooney, T. A. (2020). Impulsive pile driving noise elicits alarm responses in squid (Doryteuthis pealeii). Marine Pollution Bulletin, 150, 110792. https://doi.org/10.1016/j.marpolbul.2019.110792

Kaifu, K., Akamatsu, T., \& Segawa, S. (2008). Underwater sound detection by cephalopod statocyst. Fisheries Science, 74(4), 781-786. https://doi.org/10.1111/j.14442906.2008.01589.x

Kalmijn, A. J. (1988). Hydrodynamic and Acoustic Field Detection. In J. Atema, R. R. Fay, A. N. Popper, \& W. N. Tavolga (Eds.), Sensory Biology of Aquatic Animals (1st ed., pp. 84-130). New York: Springer-Verlag. https://doi.org/10.1007/978-1-4612-3714-3

Kaplan, M. B., Lammers, M. O., Zang, E., \& Aran Mooney, T. (2018). Acoustic and biological trends on coral reefs off Maui, Hawaii. Coral Reefs, 37(1), 121-133. 
https://doi.org/10.1007/s00338-017-1638-x

Kaplan, M. B., \& Mooney, T. A. (2015). Ambient noise and temporal patterns of boat activity in the US Virgin Islands National Park. Marine Pollution Bulletin, 98(1-2), 221-228. https://doi.org/10.1016/j.marpolbul.2015.06.047

Kaplan, M. B., \& Mooney, T. A. (2016). Coral reef soundscapes may not be detectable far from the reef. Scientific Reports, (April), 1-10. https://doi.org/10.1038/srep31862

Kaplan, M. B., Mooney, T. A., Partan, J., \& Solow, A. R. (2015). Coral reef species assemblages are associated with ambient soundscapes. Marine Ecology Progress Series, 533(Versluis 2000), 93-107. https://doi.org/10.3354/meps11382

Kaplan, M. B., \& Solomon, S. (2016). A coming boom in commercial shipping? The potential for rapid growth of noise from commercial ships by 2030. Marine Policy. https://doi.org/10.1016/j.marpol.2016.07.024

Kaschner, K. (2012). The SOUNDS table in FishBase. Retrieved from https://www.fishbase.se/Topic/List.php?group=sounds\#

Kastelein, R. A., Helder-Hoek, L., Covi, J., \& Gransier, R. (2016). Pile driving playback sounds and temporary threshold shift in harbor porpoises (Phocoena phocoena): effect of exposure duration. The Journal of the Acoustical Society of America, 139(5), 2842-2851. https://doi.org/10.1121/1.4948571

Kastelein, R. A., Helder-hoek, L., Kommeren, A., Covi, J., \& Gransier, R. (2018). Effect of piledriving sounds on harbor seal (Phoca vitulina) hearing. The Journal of the Acoustical Society of America, 143(6), 3583-3594. https://doi.org/10.1121/1.5040493

Kastelein, R. A., Jennings, N., Kommeren, A., Helder-Hoek, L., \& Schop, J. (2017). Acoustic dosebehavioral response relationship in sea bass (Dicentrarchus labrax) exposed to playbacks of pile driving sounds. Marine Environmental Research, 130, 315-324. https://doi.org/10.1016/j.marenvres.2017.08.010

Kerwin, A. H., McAnulty, S. J., \& Nyholm, S. V. (2021). Development of the accessory nidamental gland and associated bacterial community in the hawaiian bobtail squid, Euprymna scolopes. Biological Bulletin, 240(3), 205-218. https://doi.org/10.1086/713965

Kojima, T., Ito, H., Komada, T., Taniuchi, T., \& Akamatsu, T. (2005). Measurements of auditory sensitivity in common carp Cyprinus carpio by the auditory brainstem response technique and cardiac conditioning method. Fisheries Science, 71(1), 95-100. https://doi.org/10.1111/j.1444-2906.2005.00935.x

Komak, S., Boal, J. G., Dickel, L., \& Budelmann, B. U. (2005). Behavioural responses of juvenile cuttlefish (Sepia officinalis) to local water movements. Marine and Freshwater Behaviour and Physiology, 38(2), 117-125. https://doi.org/10.1080/10236240500139206

Koschinski, S., \& Lüdemann, K. (2020). Noise mitigation for the construction of increasingly large offshore wind turbines: technical options for complying with noise limits. Federal Agency for Nature Conservation, Isle of Vilm, Germany. Retrieved from https://www.bfn.de/fileadmin/BfN/meeresundkuestenschutz/Dokumente/Noisemitigation-for-the-construction-of-increasingly-large-offshore-wind-turbines.pdf

Kunc, H. P., Lyons, G. N., Sigwart, J. D., McLaughlin, K. E., \& Houghton, J. D. R. (2014). Anthropogenic noise affects behavior across sensory modalities. American Naturalist, 184(4), E93-100. https://doi.org/10.1086/677545

Ladich, F. (2019). Ecology of sound communication in fishes. Fish and Fisheries, 20, 552-563. 
https://doi.org/10.1111/faf.12368

Ladich, F., \& Fay, R. R. (2013). Auditory evoked potential audiometry in fish. Reviews in Fish Biology and Fisheries, 23(3), 317-364. https://doi.org/10.1007/s11160-012-9297-z

Lillis, A., Apprill, A., Suca, J. J., Becker, C., Llopiz, J. K., \& Mooney, T. A. (2018). Soundscapes influence the settlement of the common Caribbean coral Porites astreoides irrespective of light conditions. Royal Society Open Science, 5, 181358. https://doi.org/10.1098/rsos.181358

Lillis, A., Bohnenstiehl, D., Peters, J. W., \& Eggleston, D. (2016). Variation in habitat soundscape characteristics influences settlement of a reef-building coral. PeerJ, 4, e2557. https://doi.org/10.7717/peerj.2557

Lillis, A., Caruso, F., Mooney, T. A., Llopiz, J. K., Bohnenstiehl, D., \& Eggleston, D. B. (2018). Drifting hydrophones as an ecologically meaningful approach to underwater soundscape measurement in coastal benthic habitats. Journal of Ecoacoustics, 2, \#STBDH1. https://doi.org/10.22261/JEA.STBDH1

Lillis, A., Eggleston, D. B., \& Bohnenstiehl, D. R. (2013). Oyster larvae settle in response to habitat-associated underwater sounds. PLOS ONE, 8(10), 21-23. https://doi.org/10.1371/journal.pone.0079337

Lillis, A., \& Mooney, T. A. (2018). Snapping shrimp sound production patterns on Caribbean coral reefs: relationships with celestial cycles and environmental variables. Coral Reefs, 37(2), 1-11. https://doi.org/10.1007/s00338-018-1684-z

Lillis, A., Perelman, J. N., Panyi, A., \& Mooney, T. A. (2017). Sound production patterns of bigclawed snapping shrimp (Alpheus spp.) are influenced by time-of-day and social context. The Journal of the Acoustical Society of America, 142(5), 3311-3320. https://doi.org/10.1121/1.5012751

Lin, Y.-T., Newhall, A. E., Miller, J. H., Potty, G. R., \& Vigness-raposa, K. J. (2019). A threedimensional underwater sound propagation model for offshore wind farm noise prediction. The Journal of the Acoustical Society of America, 145(5), EL335-EL340. https://doi.org/10.1121/1.5099560

Lippert, S., \& von Estorff, O. (2019). Offshore pile driving noise: capability of numerical prediction models and ways to consider new technologies. In Zingoni (Ed.), Advances in Engineering Materials, Structures and Systems: Innovations, Mechanics and Applications (pp. 103-108). London: Taylor \& Francis Group.

Lippert, T., \& von Estorff, O. (2014). The significance of parameter uncertainties for the prediction of offshore pile driving noise. The Journal of the Acoustical Society of America, 136(5), 2463-2471. https://doi.org/10.1121/1.4896458

Lobel, P. S., Kaatz, I. M., \& Rice, A. N. (2010). Acoustical behavior of coral reef fishes. In K. Cole (Ed.), Reproduction and Sexuality in Marine Fishes (pp. 307-333). Berkeley and Los Angeles, CA: University of California Press. https://doi.org/10.1525/california/9780520264335.003.0010

Long, T., Halon, R., ter Maat, A., \& Pinsker, H. (1989). Non-associative learning in the squid Lolliguncula brevis (Mollusca, Cephalopoda). Marine Behaviour and Physiology, 16(1), 1-9.

Lugli, M., \& Fine, M. L. (2007). Stream ambient noise, spectrum and propagation of sounds in the goby Padogobius martensii: sound pressure and particle velocity. The Journal of the Acoustical Society of America, 122(5), 2881-2892. https://doi.org/10.1121/1.2783113 
Lumsdon, A. E., Artamonov, I., Bruno, M. C., Righetti, M., Tockner, K., Tonolla, D., \& Zarfl, C. (2018). Soundpeaking - hydropeaking induced changes in river soundscapes. River Research and Applications, 34, 3-12. https://doi.org/10.1002/rra.3229

Lydersen, S., Fagerland, M. W., \& Laake, P. (2009). Recommended tests for association in $2 \times 2$ tables. Statistics In Medicine, 28, 1159-1175. https://doi.org/10.1002/sim

Macy, W. K. (1982). Feeding patterns of the long-finned squid, Loligo pealei, in New England waters. Biological Bulletin, 162(1), 28-38. Retrieved from https://www.jstor.org/stable/1540967

Macy, W. K., \& Brodziak, J. K. T. (2001). Seasonal maturity and size at age of Loligo pealeii in waters of southern New England. ICES Journal of Marine Science, 58, 852-864. https://doi.org/10.1006/jmsc.2001.1076

Madsen, P. T., Wahlberg, M., Tougaard, J., Lucke, K., \& Tyack, P. L. (2006). Wind turbine underwater noise and marine mammals: Implications of current knowledge and data needs. Marine Ecology Progress Series, 309(Tyack 1998), 279-295. https://doi.org/10.3354/meps309279

Magnhagen, C., Johansson, K., \& Sigray, P. (2017). Effects of motorboat noise on foraging behaviour in Eurasian perch and roach: a field experiment. Marine Ecology, 564, 115-125. https://doi.org/10.3354/meps11997

Mangiafico, S. (2017). rcompanion: functions to support extension education program evaluation. Retrieved from https://cran.r-project.org/package=rcompanion

MARCO. (2021). Mid-Atlantic ocean data portal. Retrieved March 31, 2021, from https://portal.midatlanticocean.org/

Maruska, K. P., \& Tricas, T. C. (2009). Encoding properties of auditory neurons in the brain of a soniferous damselfish: response to simple tones and complex conspecific signals. Journal of Comparative Physiology A: Neuroethology, Sensory, Neural, and Behavioral Physiology, 195(11), 1071-1088. https://doi.org/10.1007/s00359-009-0480-1

Mather, J. A. (1991). Navigation by spatial memory and use of visual landmarks in octopuses. Journal of Comparative Physiology A, 168(4), 491-497. https://doi.org/10.1007/BF00199609

Mather, J. A., \& Kuba, M. J. (2013). The cephalopod specialties: complex nervous system, learning, and cognition. Canadian Journal of Zoology, 91(6), 431-449. https://doi.org/10.1139/cjz-2013-0009

Maximino, C., de Brito, T. M., da Silva Batista, A. W., Herculano, A. M., Morato, S., \& Gouveia Jr., A. (2010). Measuring anxiety in zebrafish: a critical review. Behavioural Brain Research, 214(2), 157-171. https://doi.org/10.1016/j.bbr.2010.05.031

Maxwell, M. R., \& Hanlon, R. T. (2000). Female reproductive output in the squid Loligo pealeii: multiple egg clutches and implications for a spawning strategy. Marine Ecology Progress Series, 199, 159-170. https://doi.org/10.3354/meps199159

Maxwell, M. R., Macy, W. K., Odate, S., \& Hanlon, R. T. (1998). Evidence for multiple spawning by squids (Loligo pealei) in captivity. Biological Bulletin, 195, 225-226. https://doi.org/10.2307/1542851

McCormick, M. I., Allan, B. J. M., Harding, H., \& Simpson, S. D. (2018). Boat noise impacts risk assessment in a coral reef fish but effects depend on engine type. Scientific Reports, $8(1)$, 1-11. https://doi.org/10.1038/s41598-018-22104-3 
McDonald, M. a, Hildebrand, J. a, \& Wiggins, S. M. (2006). Increases in deep ocean ambient noise in the Northeast Pacific west of San Nicolas Island, California. Journal of the Acoustical Society of America, 120(2), 711-718. https://doi.org/10.1121/1.2216565

McLaughlin, K. E., \& Kunk, H. P. (2015). Changes in the acoustic environment alter the foraging and sheltering behaviour of the cichlid Amititlania nigrofasciata. Behavioural Processes, 116, 75-79. Retrieved from http://dx.doi.org/10.1016/j.beproc.2015.04.012

Mensinger, A. F., Putland, R. L., \& Radford, C. A. (2018). The effect of motorboat sound on Australian snapper Pagrus auratus inside and outside a marine reserve. Ecology and Evolution, 8, 6438-6448. https://doi.org/10.1002/ece3.4002

Miksis-Olds, J. L., Bradley, D. L., \& Niu, X. M. (2013). Decadal trends in Indian Ocean ambient sound. The Journal of the Acoustical Society of America, 134(5). https://doi.org/10.1121/1.4821537

Miller, J. H., Potty, G. R., \& Kim, H. (2016). pile-driving pressure and particle velocity at the seabed: quantifying effects on crustaceans and groundfish. In A. N. Popper \& A. Hawkins (Eds.), The Effects of Noise on Aquatic Life II (pp. 719-728). New York: Springer Science+Business Media. https://doi.org/10.1007/978-1-4939-2981-8

Miller, P. J. O., Kvadsheim, P. H., Lam, F. P. A., Tyack, P. L., Curé, C., DeRuiter, S. L., Kleivane, L., Sivle, L. D., van ljsselmuide, S. P., Visser, F., Wensveen, P. J., von Benda-Beckmann, A. M., Marín López, L. M., Narazaki, T., \& Hooker, S. K. (2015). First indications that northern bottlenose whales are sensitive to behavioural disturbance from anthropogenic noise. Royal Society Open Science, 2(140484). https://doi.org/http://dx.doi.org/10.1098/rsos.140484 L.

Mogdans, J. (2019). Sensory ecology of the fish lateral-line system: morphological and physiological adaptations for the perception of hydrodynamic stimuli. Journal of Fish Biology, 95, 53-72. https://doi.org/10.1111/jfb.13966

Mollica, N. R., Guo, W., Cohen, A. L., Huang, K. F., Foster, G. L., Donald, H. K., \& Solow, A. R. (2018). Ocean acidification affects coral growth by reducing skeletal density. Proceedings of the National Academy of Sciences of the United States of America, 115(8), 1754-1759. https://doi.org/10.1073/pnas.1712806115

Montague, T. G., Rieth, I. J., \& Axel, R. (2021). Embryonic development of the camouflaging dwarf cuttlefish, Sepia bandensis. Developmental Dynamics, (December 2020), 1-16. https://doi.org/10.1002/dvdy.375

Montgomery, J. C., Jeffs, A., Simpson, S. D., Meekan, M., \& Tindle, C. (2006). Sound as an orientation cue for the pelagic larvae of reef fishes and decapod crustaceans. Advances in Marine Biology, 51(06), 143-196. https://doi.org/10.1016/S0065-2881(06)51003-X

Mooney, T. A., Andersson, M. H., \& Stanley, J. (2020). Acoustic impacts of offshore wind energy on fishery resources. Oceanography, 33(4), 82-95.

Mooney, T. A., Castellote, M., Jones, I. T., Quakenbush, L., Hobbs, R., Gaglione, E., \& Goertz, C. (2018). Local acoustic habitat relative to hearing sensitivities in beluga whales (Delphinapterus leucas). Journal of Ecoacoustics, 2(\#QZD9Z5), 1-12. https://doi.org/10.22261/JEA.QZD9Z5

Mooney, T. A., Hanlon, R. T., Christensen-Dalsgaard, J., Madsen, P. T., Ketten, D. R., \& Nachtigall, P. E. (2010). Sound detection by the longfin squid (Loligo pealeii) studied with auditory evoked potentials: sensitivity to low-frequency particle motion and not pressure. 
The Journal of Experimental Biology, 213, 3748-3459. https://doi.org/10.1242/jeb.048348 Mooney, T. A., Iorio, L. Di, Lammers, M., Lin, T., Nedelec, S. L., Parsons, M., Radford, C., Urban, E., \& Stanley, J. (2020). Listening forward: approaching marine biodiversity assessments using acoustic methods. Royal Society Open Science, 7, 201287.

https://doi.org/http://dx.doi.org/10.1098/rsos.201287

Mooney, T. A., Kaplan, M. B., \& Lammers, M. O. (2016). Singing whales generate high levels of particle motion: implications for acoustic communication and hearing? Biology Letters, 12(11), 20160381. https://doi.org/10.1098/rsbl.2016.0381

Mooney, T. A., Samson, J. E., Schlunk, A. D., \& Zacarias, S. (2016). Loudness-dependent behavioral responses and habituation to sound by the longfin squid (Doryteuthis pealeii). Journal of Comparative Physiology A: Neuroethology, Sensory, Neural, and Behavioral Physiology, 202(7), 489-501. https://doi.org/10.1007/s00359-016-1092-1

Morley, E. L., Jones, G., \& Radford, A. N. (2014). The importance of invertebrates when considering the impacts of anthropogenic noise. Proceedings of the Royal Society $B$ : Biological Sciences, 281(1776), 20132683. https://doi.org/10.1098/rspb.2013.2683

Mueller-Blenkle, C., Mcgregor, P. K., Gill, A. B., Andersson, M. H., Metcalfe, J., Bendall, V., Sigray, P., Wood, D., \& Thomsen, F. (2010). Effects of pile-driving noise on the behaviour of marine fish. Retrieved from https://tethys.pnnl.gov/sites/default/files/publications/Mueller-Benkle_et_al_2010.pdf

Murchy, K. A., Davies, H., Shafer, H., Cox, K., Nikolich, K., \& Juanes, F. (2019). Impacts of noise on the behavior and physiology of marine invertebrates: a meta-analysis. Proceedings of Meetings on Acoustics, 37, 040002. https://doi.org/10.1121/2.0001217

Musial, W., Beiter, P., Spitsen, P., Nunemaker, J., \& Gevorgian, V. (2019). 2018 offshore wind technologies market report. https://doi.org/10.2172/1572771

Musial, W., Beiter, P., Spitsen, P., Nunemaker, J., Gevorgian, V., Cooperman, A., Hammond, R., \& Shields, M. (2020). 2019 offshore wind technology data update. Retrieved from https://www.nrel.gov/wind/publications.html

Nedelec, S. L., Campbell, J., Radford, A. N., Simpson, S. D., Merchant, N. D., \& Fisher, D. (2016). Particle motion: the missing link in underwater acoustic ecology. Methods in Ecology and Evolution, 7(7), 836-842. https://doi.org/10.1111/2041-210X.12544

Nedelec, S. L., Radford, A. N., Pearl, L., Nedelec, B., Mccormick, M. I., Meekan, M. G., \& Simpson, S. D. (2017). Motorboat noise impacts parental behaviour and offspring survival in a reef fish. Proceedings of the Royal Society B: Biological Sciences, 284. https://doi.org/10.1098/rspb.2017.0143

Nedelec, S. L., Radford, A. N., Simpson, S. D., Nedelec, B., Lecchini, D., \& Mills, S. C. (2014). Anthropogenic noise playback impairs embryonic development and increases mortality in a marine invertebrate. Scientific Reports, 4, 5891. https://doi.org/10.1038/srep05891

Nedelec, S. L., Simpson, S. D., Holderied, M., Radford, A. N., Lecellier, G., Radford, C., \& Lecchini, D. (2015). Soundscapes and living communities in coral reefs: temporal and spatial variation. Marine Ecology Progress Series, 524, 125-135. https://doi.org/10.3354/meps11175

Nedwell, J. R., Parvin, S. J., Edwards, B., Workman, R., Brooker, A. G., \& Kynoch, J. E. (2007). Measurement and interpretation of underwater noise during construction and operation of offshore windfarms in UK waters. Subacoustech Report No. 544R0738 to COWRIE Ltd. 
Retrieved from www.offshorewind.co.uk

Neo, Y. Y., Hubert, J., Bolle, L., Winter, H. V, ten Cate, C., \& Slabbekoorn, H. (2016). Sound exposure changes European seabass behaviour in a large outdoor floating pen: effects of temporal structure and a ramp-up. Environmental Pollution, 214, 26-34. https://doi.org/10.1016/j.envpol.2016.03.075

Neo, Y. Y., Seitz, J., Kastelein, R. A., Winter, H. V., ten Cate, C., \& Slabbekoorn, H. (2014). Temporal structure of sound affects behavioural recovery from noise impact in European seabass. Biological Conservation, 178(June), 65-73. https://doi.org/10.1016/j.biocon.2014.07.012

Neo, Y. Y., Ufkes, E., Kastelein, R. A., Winter, H. V., ten Cate, C., \& Slabbekoorn, H. (2015). Impulsive sounds change European seabass swimming patterns: influence of pulse repetition interval. Marine Pollution Bulletin, 97(1-2), 111-117. https://doi.org/10.1016/j.marpolbul.2015.06.027

NMFS. (2021). Annual commercial landing statistics. Retrieved March 26, 2021, from https://www.fisheries.noaa.gov/foss

NRC. (2003). Ocean noise and marine mammals. National Academy of Sciences, Washington, DC: The National Academies Press. Retrieved from http://www.nap.edu/catalog/10564.html

O'Dor, R. K., Durward, R. D., \& Vessey, E. (1980). Feeding and growth in captive squid, Ilex illecebrosus, and the influence of food availability on growth in the natural population. International Commission for the Northwest Atlantic Fisheries Selected Papers, (6), 15-21.

Packard, A., Karlsen, H., \& Sand, O. (1990). Low frequency hearing in cephalopods. Journal of Comparative Physiology A, 116, 501-505.

Paris, C. B., Atema, J., Irisson, J. O., Kingsford, M., Gerlach, G., \& Guigand, C. M. (2013). Reef odor: a wake up call for navigation in reef fish larvae. PLOS ONE, 8(8), 1-8. https://doi.org/10.1371/journal.pone.0072808

Parmentier, E., Berten, L., Rigo, P., Aubrun, F., Nedelec, S. L., Simpson, S. D., \& Lecchini, D. (2015). The influence of various reef sounds on coral-fish larvae behaviour. Journal of Fish Biology, 86(5), 1507-1518. https://doi.org/10.1111/jfb.12651

Parvulescu, A. (1964). Problems of propagation and processing. In Marine bio-acoustics (pp. 87100). Bimini, Baham.

Picciulin, M., Sebastianutto, L., Codarin, A., Farina, A., \& Ferrero, E. A. (2010). In-situ behavioural responses to boat noise exposure of Gobius cruentatus (Gmelin, 1789; fam. Gobiidae) and Chromis chromis (Linnaeus, 1758; fam. Pomacentridae) living in a Marine Protected Area. Journal of Experimental Marine Biology and Ecology, 386, 125-132. https://doi.org/10.1016/j.jembe.2010.02.012

Pine, M. K., Jeffs, A. G., \& Radford, C. A. (2012). Turbine sound may influence the metamorphosis behaviour of estuarine crab megalopae. PLOS ONE, 7(12). https://doi.org/10.1371/journal.pone.0051790

Pine, M. K., Jeffs, A. G., Wang, D., \& Radford, C. A. (2016). The potential for vessel noise to mask biologically important sounds within ecologically significant embayments. Ocean and Coastal Management, 127(April), 63-73. https://doi.org/10.1016/j.ocecoaman.2016.04.007

Popper, A. N. (1972). Auditory threshold in the goldfish as a function of signal duration. The 
Journal of the Acoustical Society of America, 52(2), 596-602.

Popper, A. N., \& Fay, R. R. (1993). Sound detection and processing by fish: critical review and major research questions. Brain, Behavior and Evolution, 41, 14-38.

Popper, A. N., \& Hawkins, A. D. (2018). The importance of particle motion to fishes and invertebrates. The Journal of the Acoustical Society of America, 143(1), 470-488. https://doi.org/10.1121/1.5021594

Popper, A. N., \& Hawkins, A. D. (2019). An overview of fish bioacoustics and the impacts of anthropogenic sounds on fishes. Journal of Fish Biology, 94, 692-713. https://doi.org/10.1111/jfb.13948

Popper, A. N., Hawkins, A. D., Fay, R. R., Mann, D. A., Bartol, S., Carlson, T. J., Coombs, S., Ellison, W. T., Gentry, R. L., Halvorsen, M. B., Løkkeborg, S., Rogers, P. H., Southall, B. L., Zeddies, D. G., \& Tavolga, W. N. (2014). Sound Exposure Guidelines for Fishes and Sea Turtles: A Technical Report prepared by ANSI-Accredited Standards Committee S3/SC1 and registered with ANSI. Cham, Switzerland: SpringerBriefs in Oceanography, Springer International Publishing, and ASA Press. https://doi.org/10.1007/978-3-319-06659-2

Popper, A. N., Hawkins, A. D., Sand, O., \& Sisneros, J. A. (2019). Examining the hearing abilities of fishes. The Journal of the Acoustical Society of America, 146(948). https://doi.org/10.1121/1.5120185

Popper, A. N., Salmon, M., \& Horch, K. W. (2001). Acoustic detection and communication by decapod crustaceans. Journal of Comparative Physiology - A Sensory, Neural, and Behavioral Physiology, 187(2), 83-89. https://doi.org/10.1007/s003590100184

Popper, A. N., Smith, M. E., Cott, P. A., Hanna, B. W., MacGillivray, A. O., Austin, M. E., \& Mann, D. A. (2005). Effects of exposure to seismic airgun use on hearing of three fish species. The Journal of the Acoustical Society of America, 117(6), 3958-3971. https://doi.org/10.1121/1.1904386

Purser, J., Bruintjes, R., Simpson, S. D., \& Radford, A. N. (2016). Condition-dependent physiological and behavioural responses to anthropogenic noise. Physiology and Behavior, 155, 157-161. https://doi.org/10.1016/j.physbeh.2015.12.010

Purser, J., \& Radford, A. N. (2011). Acoustic noise induces attention shifts and reduces foraging performance in three-spined sticklebacks (Gasterosteus aculeatus). PLOS ONE, 6(2). https://doi.org/10.1371/journal.pone.0017478

Putland, R. L., Montgomery, J. C., \& Radford, C. A. (2019). Ecology of fish hearing. Journal of Fish Biology, 95, 39-52. https://doi.org/10.1111/jfb.13867

R Core Team. (2017). R: A language and environment for statistical computing. Vienna, Austria: R Foundation for Statistical COmputing. Retrieved from https://www.r-project.org/

Radford, C. A., Montgomery, J. C., Caiger, P., \& Higgs, D. M. (2012). Pressure and particle motion detection thresholds in fish: a re-examination of salient auditory cues in teleosts. The Journal of Experimental Biology, 215(19), 3429-3435. https://doi.org/10.1242/jeb.073320

Radford, C. A., Stanley, J. A., \& Jeffs, A. G. (2014). Adjacent coral reef habitats produce different underwater sound signatures. Marine Ecology Progress Series, 505, 19-28. https://doi.org/10.3354/meps10782

Radford, C. A., Stanley, J. A., Simpson, S. D., \& Jeffs, A. G. (2011). Juvenile coral reef fish use sound to locate habitats. Coral Reefs, 30(2), 295-305. https://doi.org/10.1007/s00338- 
010-0710-6

Radford, C. A., Tay, K., \& Goeritz, M. L. (2016). Hearing in the paddle crab, Ovalipes catharus. Proceedings of Meetings on Acoustics, 27(010013). https://doi.org/10.1121/2.0000259

Radford, C. A., Tindle, C. T., Montgomery, J. C., \& Jeffs, A. G. (2011). Modelling a reef as an extended sound source increases the predicted range at which reef noise may be heard by fish larvae, 438, 167-174. https://doi.org/10.3354/meps09312

Raick, X., Di lorio, L., Gervaise, C., Lossent, J., Lecchini, D., \& Parmentier, É. (2021). From the reef to the ocean: revealing the acoustic range of the biophony of a coral reef (Moorea Island, French Polynesia). Journal of Marine Science and Engineering, 9(4). https://doi.org/10.3390/jmse9040420

Reinhall, P. G., \& Dahl, P. (2011). Underwater Mach wave radiation from impact pile driving: theory and observation. The Journal of the Acoustical Society of America, 130(1209), 12091216. https://doi.org/10.1121/1.3614540

Roberts, A. (2020). Lethal coral disease spreads from St. Thomas to St. John. Retrieved from https://stthomassource.com/content/2020/06/18/lethal-coral-disease-spreads-from-stthomas-to-st-john/

Roberts, L., Cheesman, S., Breithaupt, T., \& Elliott, M. (2015). Sensitivity of the mussel Mytilus edulis to substrate-borne vibration in relation to anthropogenically generated noise. Marine Ecology Progress Series, 538, 185-195. https://doi.org/10.3354/meps11468

Roberts, L., Cheesman, S., Elliott, M., \& Breithaupt, T. (2016). Sensitivity of Pagurus bernhardus (L.) to substrate-borne vibration and anthropogenic noise. Journal of Experimental Marine Biology and Ecology, 474, 185-194. https://doi.org/10.1016/j.jembe.2015.09.014

Roberts, L., \& Elliott, M. (2017). Good or bad vibrations? Impacts of anthropogenic vibration on the marine epibenthos. Science of the Total Environment, 595, 255-268. https://doi.org/10.1016/j.scitotenv.2017.03.117

Roberts, L., \& Laidre, M. E. (2019). Finding a home in the noise: cross-modal impact of anthropogenic vibration on animal search behaviour. Biology Open, 8. https://doi.org/10.1242/bio.041988

Robinson, S. P., Lepper, P. A., \& Hazelwood, R. A. (2014). Good Practice Guide for Underwater Noise Measurement. NPL Good Practice Guide No. 133, (133).

Rogers, P. H., \& Cox, M. (1988). Underwater sound as a biological stimulus. In J. Atema, R. R. Fay, A. N. Popper, \& W. N. Tavolga (Eds.), Sensory Biology of Aquatic Animals (1st ed., pp. 131-149). New York: Springer-Verlag. https://doi.org/10.1007/978-1-4612-3714-3

Rogers, P. H., Hawkins, A. D., Popper, A. N., Fay, R. R., \& Gray, M. D. (2016). Parvulescu revisited: small tank acoustics for bioacousticians. In A. N. Popper \& A. D. Hawkins (Eds.), The Effects of Noise on Aquatic Life II (pp. 933-941). New York: Springer Science+Business Media.

Sabet, S. S., Neo, Y. Y., \& Slabbekoorn, H. (2015). The effect of temporal variation in sound exposure on swimming and foraging behaviour of captive zebra fish. Animal Behaviour, 107, 49-60. https://doi.org/10.1016/j.anbehav.2015.05.022

Salas, A., Altieri, A., Wilson, P., \& Keitt, T. (2018). Predicting the reef acoustic cuescape from the perspective of larval fishes across a habitat quality gradient. Marine Ecology Progress Series, 605, 173-193. https://doi.org/10.3354/meps12737

Samson, J. E., Mooney, T. A., Gussekloo, S. W. S., \& Hanlon, R. T. (2014). Graded behavioral 
responses and habituation to sound in the common cuttlefish Sepia officinalis. The Journal of Experimental Biology, 217(Pt 24), 4347-4355. https://doi.org/10.1242/jeb.113365

Samson, J. E., Mooney, T. A., Gussekloo, S. W. S., \& Hanlon, R. T. (2016). A brief review of cephalopod behavioral responses to sound. In A. N. Popper \& A. Hawkins (Eds.), The Effects of Noise on Aquatic Life II (pp. 969-975). New York: Springer Science+Business Media. https://doi.org/10.1007/978-1-4939-2981-8

Sand, O., Enger, P. S., Karlsen, H. E., Knudsen, F., \& Kvernstuen, T. (2000). Avoidance responses to infrasound in downstream migrating European silver eels, Anguilla anguilla. Environmental Biology of Fishes, 57, 327-336.

Sand, O., \& Karlsen, H. E. (2000). Detection of infrasound and linear acceleration in fishes. Philosophical Transactions of the Royal Society B: Biological Sciences, 355, 1295-1298. https://doi.org/10.1098/rstb.2000.0687

Scatà, G., Jozet-Alves, C., Thomasse, C., Josef, N., \& Shashar, N. (2016). Spatial learning in the cuttlefish Sepia officinalis: preference for vertical over horizontal information. Journal of Experimental Biology, 219, 2928-2933. https://doi.org/10.1242/jeb.129080

Schnell, A. K., Boeckle, M., Rivera, M., Clayton, N. S., \& Hanlon, R. T. (2021). Cuttlefish exert selfcontrol in a delay of gratification task. Proceedings of the Royal Society B: Biological Sciences, 288. https://doi.org/10.1098/rspb.2020.3161

Shashar, N., \& Hanlon, R. T. (2013). Spawning behavior dynamics at communal egg beds in the squid Doryteuthis (Loligo) pealeii. Journal of Experimental Marine Biology and Ecology, 447, 65-74. https://doi.org/10.1016/j.jembe.2013.02.011

Sigray, P., \& Andersson, M. H. (2011). Particle motion measured at an operational wind turbine in relation to hearing sensitivity in fish. The Journal of the Acoustical Society of America, 130(1), 200-207. https://doi.org/10.1121/1.3596464

Simpson, S. D., Purser, J., \& Radford, A. N. (2015). Anthropogenic noise compromises antipredator behaviour in European eels. Global Change Biology, 21(2), 586-593. https://doi.org/10.1111/gcb.12685

Simpson, S. D., Radford, A. N., Holles, S., Ferarri, M. C. O., Chivers, D. P., McCormick, M. I., \& Meekan, M. G. (2016). Small-boat noise impacts natural settlement behavior of coral reef fish larvae. In A. N. Popper \& A. Hawkins (Eds.), The Effects of Noise on Aquatic Life II (pp. 1041-1048). New York: Springer Science+Business Media. https://doi.org/10.1007/978-14939-2981-8_129

Sisneros, J.A., \& Rogers, P. H. (2016). Directional hearing and sound source localization in fishes. In Fish Hearing and Bioacoustics (Vol. 877, pp. 121-155). https://doi.org/10.1007/978-3319-21059-9_7

Slabbekoorn, H., Bouton, N., Opzeeland, I. Van, Coers, A., Cate, C., \& Popper, A. N. (2010). A noisy spring: the impact of globally rising underwater sound levels on fish. Trends in Ecology \& Evolution, 25(7), 419-427. https://doi.org/10.1016/j.tree.2010.04.005

Solé, M., Lenoir, M., Durfort, M., López-Bejar, M., Lombarte, A., \& André, M. (2013). Ultrastructural damage of Loligo vulgaris and Illex coindetii statocysts after low frequency sound exposure. PLOS ONE, 8(10), 1-12. https://doi.org/10.1371/journal.pone.0078825

Solé, M., Lenoir, M., Fortuño, J.-M., Schaar, M. Van Der, \& André, M. (2018). A critical period of susceptibility to sound in the sensory cells of cephalopod hatchlings. Biology Open, 7. https://doi.org/10.1242/bio.033860 
Solé, M., Monge, M., \& André, M. (2019). A proteomic analysis of the statocyst endolymph in common cuttlefish (Sepia officinalis): an assessment of acoustic trauma after exposure to sound. Scientific Reports, 9, 1-12. https://doi.org/10.1038/s41598-019-45646-6

Solé, M., Sigray, P., Lenoir, M., van der Schaar, M., Lalander, E., \& André, M. (2017). Offshore exposure experiments on cuttlefish indicate received sound pressure and particle motion levels associated with acoustic trauma. Scientific Reports, 7(45899). https://doi.org/10.1038/srep45899

Spiga, I., Aldred, N., \& Caldwell, G. S. (2017). Anthropogenic noise compromises the antipredator behaviour of the European seabass, Dicentrarchus labrax (L.). Marine Pollution Bulletin, 122(1-2), 297-305. https://doi.org/10.1016/j.marpolbul.2017.06.067

Spiga, I., Caldwell, G. S., \& Bruintjes, R. (2016). Influence of pile driving on the clearance rate of the blue mussel, Mytilus edulis (L.). Proceedings of Meetings on Acoustics, 27(040005). https://doi.org/10.1121/2.0000277

Staaterman, E., Ogburn, M. B., Altieri, A. H., Brandl, S. J., Whippo, R., Seemann, J., Goodison, M., \& Duffy, J. E. (2017). Bioacoustic measurements complement visual biodiversity surveys: preliminary evidence from four shallow marine habitats. Marine Ecology Progress Series, 575, 207-215. https://doi.org/10.3354/meps12188

Staaterman, E., Paris, C. B., DeFerrari, H. A., Mann, D. A., Rice, A. N., \& D’Alessandro, E. K. (2014). Celestial patterns in marine soundscapes. Marine Ecology Progress Series, 508, 1732. https://doi.org/10.3354/meps10911

Stanley, J. A., Caiger, P. E., Phelan, B., Shelledy, K., Mooney, T. A., \& Parijs, S. M. Van. (2020). Ontogenetic variation in the hearing sensitivity of black sea bass (Centropristis striata) and the implications of anthropogenic sound on behavior and communication. Journal of Experimental Biology, 223. https://doi.org/10.1242/jeb.219683

Stanley, J. A., Parijs, S. M. Van, \& Hatch, L. T. (2017). Underwater sound from vessel traffic reduces the effective communication range in Atlantic cod and haddock. Scientific Reports, 7(14633). https://doi.org/10.1038/s41598-017-14743-9

Stanley, J. A., Radford, C. A., \& Jeffs, A. G. (2010). Induction of settlement in crab megalopae by ambient underwater reef sound. Behavioral Ecology, 21(1), 113-120. https://doi.org/10.1093/beheco/arp159

Stanley, J. A., Radford, C. A., \& Jeffs, A. G. (2011). Behavioural response thresholds in New Zealand crab megalopae to ambient underwater sound. PLOS ONE, 6(12). https://doi.org/10.1371/journal.pone.0028572

Stanley, J. A., Radford, C. A., \& Jeffs, A. G. (2012). Location, location, location: finding a suitable home among the noise. Proceedings of the Royal Society B: Biological Sciences, 279(1742), 3622-3631. https://doi.org/10.1098/rspb.2012.0697

Staudinger, M. D., Hanlon, R. T., \& Juanes, F. (2011). Primary and secondary defences of squid to cruising and ambush fish predators: variable tactics and their survival value. Animal Behaviour, 81, 585-594. https://doi.org/10.1016/j.anbehav.2010.12.002

Suca, J. J., Lillis, A., Jones, I. T., Kaplan, M. B., Solow, A. R., Earl, A. D., Habtes, S., Apprill, A., Llopiz, J. K., \& Mooney, T. A. (2020). Variable and spatially explicit response of fish larvae to the playback of local, continuous reef soundscapes. Marine Ecology Progress Series, 653, 131-151. https://doi.org/10.3354/meps13480

Tougaard, J., Carstensen, J., Teilmann, J., Skov, H., \& Rasmussen, P. (2009). Pile driving zone of 
responsiveness extends beyond $20 \mathrm{~km}$ for harbor porpoises (Phocoena phocoena (L.)). The Journal of the Acoustical Society of America, 11. https://doi.org/10.1121/1.3132523

Tricas, T. C., \& Boyle, K. S. (2014). Acoustic behaviors in Hawaiian coral reef fish communities. Marine Ecology Progress Series, 511, 1-16. https://doi.org/10.3354/meps10930

Tricas, T. C., \& Webb, J. F. (2016). Acoustic communication in butterflyfishes: anatomical novelties, physiology, evolution, and behavioral ecology. In Joseph A Sisneros (Ed.), Fish Hearing and Bioacoustics (pp. 57-92). Springer International Publishing Switzerland. https://doi.org/10.1007/978-3-319-21059-9

Tsounis, G., \& Edmunds, P. J. (2017). Three decades of coral reef community dynamics in St. John, USVI: a contrast of scleractinians and octocorals. Ecosphere, 8(1). https://doi.org/10.1002/ecs2.1646

Tsouvalas, A., \& Metrikine, A. V. (2016). Structure-borne wave radiation by impact and vibratory piling in offshore installations: from sound prediction to auditory damage. Journal of Marine Science and Engineering, 3(44). https://doi.org/10.3390/jmse4030044

UCLA Statistical Consulting Group. (n.d.). FAQ: What are pseudo R-squareds? Retrieved January 11,2018 , from https://web.archive.org/web/20130701052125/http://www.ats.ucla.edu:80/stat/mult_pk $\mathrm{g} / \mathrm{faq} /$ general/citingats.htm

Vermeij, M. J. A., Marhaver, K. L., Huijbers, C. M., Nagelkerken, I., \& Simpson, S. D. (2010). Coral larvae move toward reef sounds. PLOS ONE, 5(5), 3-6. https://doi.org/10.1371/journal.pone.0010660

Villalobos, G., Alison, J., \& Christopher, M. D. (2017). Environmental noise reduces predation rate in an aquatic invertebrate. Journal of Insect Conservation, 21(5), 839-847. https://doi.org/10.1007/s10841-017-0023-y

Vovk, A. N. (1985). Feeding spectrum of longfln Squid (Loligo pealei) in the Northwest Atlantic and its position in the ecosystem. NAFO Scientific Council Studies, 8, 33-38.

Wahlberg, M., Schack, H. B., Wilson, M., Bejder, L., \& Madsen, P. (2008). Particle acceleration noise generated by boats. Bioacoustics, 17(1-3), 148-150. https://doi.org/10.1080/09524622.2008.9753797

Wahlberg, M., \& Westerberg, H. (2005). Hearing in fish and their reactions to sounds from offshore wind farms. Marine Ecology Progress Series, 288, 295-309.

Wale, M. A., Simpson, S. D., \& Radford, A. N. (2013a). Noise negatively affects foraging and antipredator behaviour in shore crabs. Animal Behaviour, 86(1), 111-118. https://doi.org/10.1016/j.anbehav.2013.05.001

Wale, M. A., Simpson, S. D., \& Radford, A. N. (2013b). Size-dependent physiological responses of shore crabs to single and repeated playback of ship noise. Biology Letters, 9(2), 20121194. https://doi.org/10.1098/rsbl.2012.1194

Webster, D B, Fay, R. R., \& Popper, A. N. (1992). The Evolutionary Biology of Hearing. (Douglas B Webster, R. R. Fay, \& A. N. Popper, Eds.). New York: Springer-Verlag.

Wells, M. J., \& Wells, J. (1956). The function of the brain of octopus in tactile discrimination. Journal of Experimental Biology, 34(November), 131-142.

Wilson, M., Haga, J. Å. R., \& Karlsen, H. E. (2018). Behavioural responses to infrasonic particle acceleration in cuttlefish. The Journal of Experimental Biology, 221(1), jeb166074. https://doi.org/10.1242/jeb.166074 
Wingfield, J. C., \& Sapolsky, R. M. (2003). Reproduction and resistance to stress: when and how. Journal of Neuroendocrinology, 15, 711-724. https://doi.org/10.1046/j.13652826.2003.01033.x

Wood, J. B., Maynard, A. E., Lawlor, A. G., Sawyer, E. K., Simmons, D. M., Pennoyer, K. E., \& Derby, C. D. (2010). Caribbean reef squid, Sepioteuthis sepioidea, use ink as a defense against predatory French grunt, Haemulon flavolineatum. Journal of Experimental Marine Biology and Ecology, 388, 20-27. https://doi.org/10.1016/j.jembe.2010.03.010

Wood, J. B., Pennoyer, K. E., \& Derby, C. D. (2008). Ink is a conspecific alarm cue in the Caribbean reef squid, Sepioteuthis sepioidea. Journal of Experimental Marine Biology and Ecology, 367, 11-16. https://doi.org/10.1016/j.jembe.2008.08.004

Wright, K. J., Higgs, D. M., \& Leis, J. M. (2011). Ontogenetic and interspecific variation in hearing ability in marine fish larvae. Marine Ecology Progress Series, 424, 1-13. https://doi.org/10.3354/meps09004

Wysocki, L. E., Codarin, A., Ladich, F., \& Picciulin, M. (2009). Sound pressure and particle acceleration audiograms in three marine fish species from the Adriatic Sea. The Journal of the Acoustical Society of America, 126(4), 2100. https://doi.org/10.1121/1.3203562

Wysocki, L. E., \& Ladich, F. (2003). The representation of conspecific sounds in the auditory brainstem of teleost fishes. Journal of Experimental Biology, 206(13), 2229-2240. https://doi.org/10.1242/jeb.00417

Wysocki, L. E., \& Ladich, F. (2005). Hearing in fishes under noise conditions. JARO - Journal of the Association for Research in Otolaryngology, 6(1), 28-36. https://doi.org/10.1007/s10162-004-4043-4

York, C. A., \& Bartol, I. K. (2014). Lateral line analogue aids vision in successful predator evasion for the brief squid, Lolliguncula brevis. The Journal of Experimental Biology, 217, 24372439. https://doi.org/10.1242/jeb.102871

York, C. A., Bartol, I. K., \& Krueger, P. S. (2016). Multiple sensory modalities used by squid in successful predator evasion throughout ontogeny. The Journal of Experimental Biology, (July), jeb.140780. https://doi.org/10.1242/jeb.140780

Zakroff, C. J., \& Mooney, T. A. (2020). Antagonistic interactions and clutch-dependent sensitivity induce variable responses to ocean acidification and warming in squid (Doryteuthis pealeii) embryos and paralarvae. Frontiers in Physiology, 11. https://doi.org/10.3389/fphys.2020.00501

Zeddies, D. G., Fay, R. R., Alderks, P. W., Shaub, K. S., \& Sisneros, J. a. (2010). Sound source localization by the plainfin midshipman fish, Porichthys notatus. The Journal of the Acoustical Society of America, 127(July 2015), 3104-3113. https://doi.org/10.1121/1.3384691

Zeddies, D. G., Fay, R. R., Gray, M. D., Alderks, P. W., Acob, A., \& Sisneros, J. a. (2012). Local acoustic particle motion guides sound-source localization behavior in the plainfin midshipman fish, Porichthys notatus. Journal of Experimental Biology, 215(1), 152-160. https://doi.org/10.1242/jeb.064998

Zeileis, A., Kleiber, C., \& Jackman, S. (2008). Regression models for count data in R. Journal of Statistical Software, 27(8). Retrieved from http://www.jstatsoft.org/v27/i08/

Zepeda, E. A., Veline, R. J., \& Crook, R. J. (2017). Rapid associative learning and stable long-term memory in the squid Euprymna scolopes. Biological Bulletin, 232, 212-218. 